\title{
The phylogenetics of Teleosauroidea (Crocodylomorpha, Thalattosuchia) and implications for their ecology and evolution
}

\author{
Michela M Johnson ${ }^{\text {Corresp., }}{ }^{1}$, Mark T Young ${ }^{1}$, Stephen L Brusatte ${ }^{1,2}$ \\ ${ }^{1}$ School of GeoSciences, University of Edinburgh, Edinburgh, United Kingdom \\ 2 National Museum of Scotland, Edinburgh, United Kingdom \\ Corresponding Author: Michela M Johnson \\ Email address: michela.johnson@ed.ac.uk
}

Teleosauroidea was a clade of ancient crocodylomorphs that were a key element of coastal marine environments during the Jurassic. Despite a 300-year research history and a recent renaissance in the study of their morphology and taxonomy, macroevolutionary studies of teleosauroids are currently limited by our poor understanding of their phylogenetic interrelationships. One major problem is the genus Steneosaurus, a wastebasket taxon recovered as paraphyletic or polyphyletic in phylogenetic analyses. We constructed a newly updated phylogenetic data matrix containing 153 taxa (27 teleosauroids, eight of which were newly added) and 502 characters, which we analysed under maximum parsimony using TNT 1.5 (weighted and unweighted analyses) and Bayesian inference using MrBayes v3.2.6 (standard, gamma, and variation). The resulting topologies were then analysed to generate comprehensive higher-level phylogenetic hypotheses of teleosauroids and shed light on species-level interrelationships within the clade. The results from our parsimony and Bayesian analyses are largely consistent. Two large subclades within Teleosauroidea are recovered, and they are morphologically, ecologically and biogeographically distinct from one another. Based on comparative anatomical and phylogenetic results, we propose the following major taxonomic revisions to Teleosauroidea: (1) redefining Teleosauridae; (2) introducing one new family and three new subfamilies; (3) the resurrection of three historical genera; and (4) erecting seven new generic names and one new species name. The phylogeny infers that the Laurasian subclade was more phenotypically plastic overall than the Sub-Boreal-Gondwanan subclade. The proposed phylogeny shows that teleosauroids were more diverse than previously thought, in terms of morphology, ecology, dispersal and abundance, and that they represented some of the most successful crocodylomorphs during the Jurassic. 
1 The phylogenetics of Teleosauroidea (Crocodylomorpha, Thalattosuchia) and implications for

2 their ecology and evolution

3 Michela M. Johnson ${ }^{1 *}$, Mark T. Young ${ }^{1}$, Stephen L. Brusatte ${ }^{1,2}$

4

5

$6 \quad{ }^{1}$ School of GeoSciences, University of Edinburgh, Edinburgh, United Kingdom

$7 \quad{ }^{2}$ National Museum of Scotland, Chambers Street, Edinburgh, United Kingdom

8

9

10

$11 *$ corresponding author: michela.johnson@ed.ac.uk

12 


\section{Abstract}

14 Teleosauroidea was a clade of ancient crocodylomorphs that were a key element of coastal 15 marine environments during the Jurassic. Despite a 300-year research history and a recent 16 renaissance in the study of their morphology and taxonomy, macroevolutionary studies of 17 teleosauroids are currently limited by our poor understanding of their phylogenetic

18 interrelationships. One major problem is the genus Steneosaurus, a wastebasket taxon recovered 19 as paraphyletic or polyphyletic in phylogenetic analyses. We constructed a newly updated 20 phylogenetic data matrix containing 153 taxa (27 teleosauroids, eight of which were newly 21 added) and 502 characters, which we analysed under maximum parsimony using TNT 1.5 22 (weighted and unweighted analyses) and Bayesian inference using MrBayes v3.2.6 (standard, 23 gamma, and variation). The resulting topologies were then analysed to generate comprehensive

24 higher-level phylogenetic hypotheses of teleosauroids and shed light on species-level

25 interrelationships within the clade. The results from our parsimony and Bayesian analyses are 26 largely consistent. Two large subclades within Teleosauroidea are recovered, and they are 27 morphologically, ecologically and biogeographically distinct from one another. Based on 28 comparative anatomical and phylogenetic results, we propose the following major taxonomic 29 revisions to Teleosauroidea: (1) redefining Teleosauridae; (2) introducing one new family and three new subfamilies; (3) the resurrection of three historical genera; and (4) erecting seven new generic names and one new species name. The phylogeny infers that the Laurasian subclade was

32 more phenotypically plastic overall than the Sub-Boreal-Gondwanan subclade. The proposed

33 phylogeny shows that teleosauroids were more diverse than previously thought, in terms of

34 morphology, ecology, dispersal and abundance, and that they represented some of the most 35 successful crocodylomorphs during the Jurassic. 


\section{Introduction}

37 Teleosauroid crocodylomorphs - distant extinct relatives of extant crocodylians (which include

38 alligators, crocodiles, caimans and gavials) - were a near-globally distributed clade that

39 frequented freshwater, brackish, lagoonal and deep-water marine ecosystems throughout the

40 Jurassic (Buffetaut 1982; Hua \& Buffetaut, 1997; Hua 1999; Young et al., 2014; Foffa, Young \&

41 Brusatte, 2015, 2019; Johnson et al. 2015; Martin et al. 2016; Johnson et al. 2017, 2019). They

42 have frequently been regarded as marine analogues of extant gavials, as the majority of species

43 had an elongate and tubular snout, high tooth count and dorsally directed orbits, suggestive of a

44 feeding style of catching small, fast-moving prey (Andrews, 1909, 1913; Buffetaut, 1982; Hua,

45 1999). Teleosauroids are part of the wider crocodylomorph clade Thalattosuchia, which also

46 includes the metriorhynchoids: the only archosaurs to adopt a fully pelagic, open-ocean,

47 swimming lifestyle in the manner of modern cetaceans (Young et al, 2010; Parrilla-Bel et al.,

48 2013; Foffa \& Young, 2014).

While teleosauroid skeletal and dental morphology has been well documented from the

$5018^{\text {th }}$ Century to present (Chapman, 1758; Cuvier, 1824; von Meyer, 1837; Eudes-

51 Deslongchamps, 1867-69; Blake, 1876; Andrews, 1909, 1913; Westphal, 1961, 1962; Young et

52 al., 2014; Johnson et al., 2017, 2019; Foffa et al., 2019; Sachs et al., 2019a), the evolutionary

53 relationships of these crocodylomorphs are poorly understood and little studied. This is

54 problematic, as phylogenies are crucial when evaluating evolutionary changes throughout time

55 (Purvis, Gittleman \& Brooks, 2005; Mishra \& Thines, 2014). One of the major problems in

56 teleosauroid systematics is the nomenclatural nightmare that is the taxon Steneosaurus.

57 Widespread taxonomic lumping has seen this genus become a 'wastebasket' for a multitude of 
58 species. The validity of Steneosaurus has recently been called into question (Jouve et al., 2017;

59 Johnson, Young \& Brusatte, 2020) as the type specimen of the type species, Steneosaurus

60 rostromajor Geoffroy Saint-Hilaire, 1825 (MNHN.RJN 134c-d), has rarely been referenced or

61 figured in the literature since its preliminary descriptions by Cuvier $(1800,1808,1812,1824)$

62 and Geoffroy Saint-Hilaire $(1825,1831)$. Another problematic issue reinforced during the $20^{\text {th }}$

63 Century (e.g. Andrews, 1909, 1913) is the contention that while there are noticeable differences

64 between the skulls of teleosauroid species, the postcranial skeleton only shows superficial

65 differences. This led to the assumption that teleosauroids must have lived in similar habitats with

66 a conservative body plan (Andrews, 1913; Buffetaut, 1982). However, recent studies (e.g. Young

67 et al., 2014; Johnson et al., 2017; Foffa et al., 2019; Martin et al., 2016, 2019; Wilberg, Turner \&

68 Brochu, 2019) have begun to dispute this notion, showing, in terms of postcranial anatomy and

69 palaeoenvironment, that teleosauroids were more diverse than originally thought.

70 Herein we present an in-depth, comprehensive phylogenetic study of Teleosauroidea,

71 using the most recently updated crocodylomorph dataset. We will: (1) explore the historical

72 background of teleosauroid phylogenetics; (2) discuss the materials and phylogenetic methods

73 used; (3) provide a novel, comprehensive taxonomic layout of Teleosauroidea; (4) list detailed

74 descriptions of both newly scored and morphologically important characters; (5) evaluate the

75 results of the phylogenetic analyses; and (6) elucidate what this new phylogeny implies about

76 teleosauroid ecomorphological and distributional patterns.

77

78 Historical Background 
79 1.1 Previous teleosauroid phylogenetics - late 1900s, early 2000s, and Mueller-Töwe's (2006)

80 contributions

81 Although descriptions of teleosauroid fossils were prevalent during the mid- $18^{\text {th }}$ and $19^{\text {th }}$

82 Centuries (Chapman, 1758; Morton \& Wooller, 1758; Cuvier, 1808, 1812, 1824; Geoffroy Saint-

83 Hilaire, 1825, 1831; von Meyer, 1837; Eudes-Deslonghcamps, 1867-69; Westphal, 1961),

84 investigation into their evolutionary relationships remains a relatively new area of study. While

85 Buffetaut (1980a, 1980b) and Vignaud (1995) briefly took note on the general interrelationships

86 within Thalattosuchia, Benton \& Clark (1988) examined the overall phylogenetic affinities of

87 crocodylomorphs as a group. During the early $21^{\text {st }}$ Century, thalattosuchians continued to be

88 incorporated into larger crocodylomorph studies. However, these analyses were not focused on

89 the interrelationships between thalattosuchians, and usually included only one or two

90 teleosauroid taxa, namely Steneosaurus bollensis Jäger, 1828, and Pelagosaurus typus Bronn,

91 1841, which was considered a basal teleosauroid during that time (Gasparini, Pol \& Spalletti,

92 2006; Pol \& Gasparini, 2009).

93 Mueller-Töwe's (2006) unpublished thesis included the first analysis that focused

94 specifically on thalattosuchian phylogenetics, in particular Teleosauridae, and was built upon a

95 preliminary study (Mueller-Töwe, 2005). Mueller-Töwe's (2006) dataset included 189

96 characters, with twelve teleosauroids out of 29 taxa: Machimosaurus hugii von Meyer, 1837;

97 Platysuchus multiscrobiculatus (Berckhemer, 1929) Westphal 1961; Steneosaurus baroni

98 Newton, 1983; S. bollensis; Steneosaurus edwardsi Eudes-Deslongchamps, 1868a; Steneosaurus

99 boutilieri Eudes-Deslongchamps, 1868b; Steneosaurus brevior Blake, 1876; Steneosaurus

100 gracilirostris Westphal, 1961; Steneosaurus leedsi Andrews, 1909 (which also incorporated 
101 Mycterosuchus nasutus Andrews, 1913); Steneosaurus megarhinus Hulke, 1871; Steneosaurus

102 obtusidens Andrews, 1909; Steneosaurus (Aeolodon) priscus von Sömmerring, 1814; and

103 Teleosaurus cadomensis (Lamouroux, 1820). Other taxa were considered insufficient to include

104 in the dataset (e.g. specimens that the author felt contained insufficient information and/or

105 skeletal material), and only four teleosauroids used in the analysis were studied in-depth: $P l$.

106 multiscrobiculatus, S. brevior, S. bollensis and S. gracilirostris (note that Mueller-Töwe [2006]

107 focused specifically on Toarcian species). In addition, there were no ordered or weighted

108 characters, and multi-state characters were treated as polymorphs (Mueller-Töwe, 2006).

109 Disregarding ordered or weighted characters, however, presents a problem, as ordered parsimony

110 is less artefactual and susceptible to polarization errors, and displays an overall higher

111 performance level than unordered parsimony (Grand et al., 2013; Rineau et al., 2015).

Mueller-Töwe's (2006) strict consensus topology (Fig. 1A) produced 123 most

113 parsimonious trees (MPTs) with a tree length of 423, an ensemble consistency index (CI) of

1140.6312 and an ensemble retention index $(\mathrm{RI})$ of 0.6549 . The teleosauroids were found to be

115 monophyletic and included: (1) Pel. typus as the basal-most teleosauroid; (2) a paraphyletic

116 Steneosaurus; and (3) Platysuchus as the most closely related taxon to Machimosaurus (Fig.

117 1A). However, it is important to note that in Mueller-Töwe (2006) there are several factual errors

118 and inconsistencies, particularly in the anatomical descriptions, which may have had an influence

119 on the phylogenetic results. Note that as her final analyses were not subject to peer-review

120 publication, it is unfair to give undue criticism.

When re-describing T. cadomensis, Jouve (2009) performed a phylogenetic analysis

122 consisting of 75 taxa and 343 characters, and included the teleosauroids Teleosaurus cadomensis, 
123 Peipehsuchus teleorhinus Young, 1948 (now known as the Chinese teleosauroid IVPP V 10098),

124 S. bollensis, Pel. typus (still considered to be a teleosauroid by some, although there was growing

125 support for it as a metriorhynchoid: e.g. Buffetaut, 1980a; Mercier, 1993), Steneosaurus larteti

126 Eudes-Deslongchamps, 1866a, and 'Mystriosaurus' Kaup, 1834 (= Pelagosaurus tomarensis,

127 MUHNAC unnumbered specimen: Telles-Antunes, 1967). The strict consensus (Fig. 1B) was

128 found from four MPTs. Another study (Pierce, Angielczyk \& Rayfield, 2009) conducted a

129 parsimony analysis based off Mueller-Töwe's (2006) unpublished character matrix; however,

130 species they considered synonymous (e.g. S. leedsi and S. megarhinus) were combined and taxa

131 not used in the authors' landmark-based geometric morphometric analysis were deleted.

132 Therefore, only seven teleosauroids were included (Steneosaurus heberti Morel de Glasville,

133 1876, S. gracilirostris, Pl. multiscrobiculatus, Mac. hugii, S. leedsi, S. bollensis and S. brevior),

134 as well as Pel. typus, and Metriorhynchus superciliosus de Blainville, 1853 as the outgroup

135 (Pierce, Angielczyk \& Rayfield, 2009). This dataset produced two MPTs with 115 steps (CI =

$1360.621)$

137 1.2 The leisurely rise of teleosauroid phylogenetics - post-2010

138 Bronzati, Montefeltro \& Langer (2012) presented an in-depth crocodylomorph supertree and

139 included 19 teleosauroid species in their analysis; however, the Chinese teleosaurid (IVPP V

140 10098) was attributed to the metriorhynchoid Peipehsuchus; S. edwardsi, and Steneosaurus

141 durobrivensis Andrews, 1909 (which is now considered a subjective junior synonym of $S$.

142 edwardsi; see Johnson et al. 2015) were treated as separate taxa; and Steneosaurus pictaviensis

143 Vignaud, 1998, was included (which is a subjective junior synonym of S. leedsi; see below).

144 Several key taxa were also absent in the analysis (e.g. Myc. nasutus, S. obtusidens, 
145 Machimosaurus mosae Sauvage \& Liénard, 1879). In addition, Bronzati, Montefeltro \& Langer

146 (2012) searched for their source trees on Web of Science, other Internet search engines and

147 published references, synthesizing published phylogenies and thus not personally examining the

148 specimens. The result was a major polytomy of Teleosauroidea as a whole, with 'Mystriosaurus' 149 and Pl. multiscrobiculatus unresolved at the base.

150 Wilberg (2015a) devised an updated crocodylomorph matrix (referred herein as the W matrix) which included nine teleosauroid taxa (S. brevior; Steneosaurus brevidens Phillips, 1871;

152 153 154 155

'Teleosaurus'; Mac. hugii; S. leedsi; S. durobrivensis; Pl. multiscrobiculatus; S. bollensis; and Peipehsuchus [again considered a teleosauroid]). The strict consensus topology produced 566 MPTs and 1649 steps $(\mathrm{CI}=0.312 ; \mathrm{RI}=0.703)$ and a monophyletic teleosauroid clade, which continued to be stable regardless of different constraints placed on thalattosuchians as a whole (Wilberg, 2015a). This is somewhat similar to the results seen in follow-up studies by Wilberg (2015b) (Fig. 1C), Wilberg (2017) and Wilberg, Turner \& Brochu (2019), and these produced comparable results to the recently updated Hastings+Young matrices (see below). However, there is one major change from Wilberg (2015a) to the updated results in Wilberg (2015b) and Wilberg, Turner \& Brochu (2019): Pel. typus is now moved to the base of Metriorhynchoidea. crocodylomorph, notably thalattosuchian, phylogenetics (Foffa et al., 2019; Johnson, Young \& Brusatte, 2019; Sachs et al., 2019a). In particular, a dataset known as the Hastings+Young $(\mathrm{H}+\mathrm{Y})$ dataset is being continuously updated to assess these evolutionary relationships. In 2016, Hastings and Young combined their respective crocodylomorph matrices to create this dataset, which acted as the foundation for the Crocodylomorph SuperMatrix Project. Ristevski et al. 
167 (2018), focusing on the interrelationhsips within goniopholidids, ran the first comprehensive

168 version of this dataset, which included 14 thalattosuchians and three teleosauroids ( $P l$.

169 multiscrobiculatus, S. heberti and S. bollensis). Ösi et al. (2018), describing the metriorhynchoid

170 Magyarosuchus fitosi, ran an updated version of the H+Y matrix with 140 OTUs (operational

171 taxonomic units) for 454 characters, resulting in 84 MPTs with 1477 steps. Fifteen teleosauroids

172 were included and Teleosauroidea was recovered as a monophyletic group, with $S$. gracilirostris

173 as the basal-most teleosauroid and two distinct subgroups. When re-describing 'S.' megarhinus,

174 Foffa et al. (2019) used a slightly modified version of the H+Y dataset: 140 OTUs, 18 of these

175 teleosauroid taxa, for 456 characters, producing 85 MPTs with 1494 steps $(C I=0.414, \mathrm{RI}=$

176 0.841). The strict consensus topology was similar to that found in Ösi et al (2018) (S.

177 gracilirostris as the basal taxon, two distinct subgroups), but showed different positions of

178 certain taxa, most notably Aeolodon priscus and 'Teleosaurus' [Bathysuchus] megarhinus. In

179 Johnson, Young \& Brusatte (2019) and Sachs et al. (2019a), subsequent versions of the H+Y

180 dataset were used; the phylogenetic analyses included 19 and 18 teleosauroid taxa, respectively,

181 both producing an overall similar appearance of Teleosauroidea as that of Ösi et al (2018) and

182 Foffa et al. (2019). The H+Y dataset used in Johnson, Young \& Brusatte (2019) included 143

183 OTUs for 464 characters, producing 201 MPTs with 1526 steps $(C I=0.415 ; \mathrm{RI}=0.845)$ (Fig.

184 1D), whereas Sachs et al. (2019a) produced 197 MPCs and 1513 steps $(C I=0.417$; RI $=0.846)$

185 from 142 OTUs for 462 characters.

Curiously, Martin et al. (2019) used Wilberg's (2015a) dataset, with no explanation as to

187 why they did not use one of the more recent versions of the Wilberg dataset then published

188 (Wilberg 2015b, Wilberg 2017, or the W dataset in Ösi et al. 2018) or the most currently updated

$189 \mathrm{H}+\mathrm{Y}$ matrix (provided in Foffa et al. (2019) at that time). The W dataset (Wilberg, 2015a) was 
190 also used in Martin et al. (2016), again with no clarification as to why an updated W dataset

191 (Wilberg, 2015b) was not used. Out of 78 OTUs, only 24 thalattosuchians (14 teleosauroids)

192 were included (Martin et al., 2019), with similar taxonomic concerns found in Mueller-Töwe's

193 (2006) analysis. For example, S. durobrivensis (= subjective junior synonym of S. edwardsi;

194 Johnson et al., 2015) was treated as a distinct taxon, and many distinct species were excluded

195 from the analysis. Machimosaurus buffetauti Young et al., 2015b (initially described as a valid

196 taxon in Young et al., 2014) was treated as Mac. hugii due to the monospecific hypothesis put

197 forth in Martin \& Vincent (2013) (for more information, see Foffa et al., 2019). Furthermore,

198 while I. potamosiamensis and Mac. hugii were coded in their entirety into the W matrix, three

199 characters $(174,176$, and 184) were altered from the original used by Wilberg (2015a), but only

200 for the Chinese teleosauroid (IVPP V 10098) (Martin et al., 2019). Thus, the results (12 MPTs

201 with 1666 steps) (Fig. 1E) were drastically different than those found in Wilberg (2015b), Young

202 et al. (2016), Ristevski et al. (2018), Ösi et al. (2018), Foffa et al. (2019), Johnson, Young \&

203 Brusatte (2019) and Sachs et al. (2019a).

204

205 Abbreviations

206 Institutional: BHN2, Muséum d'Histoire Naturelle de Boulogne-sur-Mer, France (closed in 207 2003); BIRUG, Lapworth Museum of Geology, Birmingham, UK; BRLSI, Bath Royal Literary 208 and Scientific Institution, Bath, UK; BSY, Catalogue du patrimoine paléontologique jurassien 209 A16, Porrentruy, Switzerland; CAMSM, Sedgwick Museum of Earth Science, Cambridge, UK;

210 DFMMh, Dinosaurier-Freilichtmuseum Münchehagen, Lower Saxony, Germany; DONMG, 211 Doncaster Museum, Doncaster, UK; DORCM, Dorset County Museum, Dorchester, UK; 
212 FMNH, Field Museum of Natural History, Chicago, USA; GPIT, Paläontologische Sammlung 213 der Eberhard Karls Universität, Tübingen, Germany; GrozNII, Grozny Petroleum Research

214 Institute, Chechen Republic, Russia; GZG, Geologisches institut Geologisch-Paläontologisches,

215 Göttingen, Germany; HLMD, Hessisches Landesmuseum, Darmstadt, Germany; IRSNB,

216 Institut Royal des Sciences Naturelles de Bruxelles, Brussels, Belgium; IVPP, Institute of

217 Paleontology and Paleoanthropology, Beijing, China; LMH, Landesmuseum, Hannover,

218 Germany; LPP, Institut de paléoprimatologie, paléontologie, humaine évolution et

219 paléoenvironnements Université de Poitiers, Poitiers, France; MCNV, Museo de Ciencias

220 Naturales de Valencia, Spain; MG, Museu Geológico, Lisbon, Portugal; ML, Museu da

221 Lourinhã, Lourinhã, Portugal; MMG, Staaliches Museum für Mineralogie und Geologie,

222 Dresden, Germany; MMT, Musée d'Art et d'Histoire Michel Hachet, Toul, France; MNHN,

223 Muséum National d'Histoire Naturelle, Paris, France; MNHNL, Musée National d'Histoire

224 naturelle, Luxembourg City, Luxembourg; MPV, Musée paléontologique (Paléospace) de

225 Villers-sur-Mer, Normandy, France; MUHNAC, Museu Nacional de História Natural e da

226 Ciência Lisbon, Lisbon, Portugal; NHMUK, Natural History Museum, London, UK; NAMU,

227 Naturkunde-Museum Bielefeld, Bielefeld, Germany; NHMW, Naturhistorisches Museum Wien,

228 Vienna, Austria; NM, Národní museum, Prague, Czech Republic; NMS, Naturmuseum

229 Solothurn, Switzerland; NMNSJ, National Museum of Nature and Science, Tokyo, Japan;

230 NOTNH, Nottingham Natural History Museum, Nottingham, UK; NZM-PZ, Naturhistoriska

231 Riksmuseet Palaeozoological, Stockholm, Sweden; ONM, Office National des Mines, Tunis,

232 Tunisia; OUMNH, Oxford University Museum of Natural History, Oxford, UK; PETMG,

233 Peterborough Museum and Art Gallery, Peterborough, UK; PIN, Paleontological Institute,

234 Moscow, Russia; PMU, Evolutionsmuseet Uppsala Universitet, Uppsala, Sweden; PRC, 
235 Palaeontological Research and Education Centre, Maha Sarakham University, Thailand; SCR,

236 Catalogue du patrimoine paléontologique jurassien - A16, Porrentruy, Switzerland; SMF,

237 Naturmuseum Senckenberg Frankfurt, Germany; SMHM, Staaliches Naturhistorisches Museum,

238 Braunschweig, Germany; SMNS, Staatliches Museum für Naturkunde Stuttgart,

239 BadenWürttemberg, Germany; TCH, Catalogue du patrimoine paléontologique jurassien - A16,

240 Porrentruy, Switzerland; UH, Urweltmuseum Hauff Holzmaden, Germany; VTT, Catalogue du

241 patrimoine paléontologique jurassien - A16, Porrentruy, Switzerland; YORYM, Yorkshire

242 Museum, York, UK.

243 Anatomical: ac, acetabulum; ?an, possible angular; an, angular; anas, anastomosing pattern

244 (tooth); ant il pr, anterior iliac process; antorb f, antorbital fenestra; art, articular; ?atl-ax,

245 possible atlas-axis complex; atl, atlas; ax, axis; basiocc, basioccipital; ?basisph, possible

246 basisphenoid; basisph, basisphenoid; cerv r, cervical rib; cerv v, cervical vertebra; cn XII,

247 cranial nerve XII; cor, coracoid; cor f, coracoid foramen; cor gr, coronoid groove; D3, third

248 dentary alveolus; D4, fourth dentary alveolus; D16, sixteenth denary alveolus; D17, seventeenth

249 dentary alveolus; den, dentary; dors os, dorsal osteoderm; dors v, dorsal vertebra; ectopt,

250 ectopterygoid; ex n, external nares; f, frontal; f $\mathbf{m}$, foramen magnum; fem, femur; fem $\mathbf{h}$,

251 femoral head; gl f, glenoid fossa; hum, humerus; hum h, humeral head; il, ilium; isch, ischium;

252 isch bl, ischial blade; j, jugal; ?l, possible lacrimal; l, lacrimal (lachrymal); k, keel (osteoderm);

253 li, limb bone (unknown); M10, tenth maxillary alveolus; M12, twelfth maxillary alveolus; mand

254 f, mandibular fenestra; mand sy, mandibular symphysis; meck c, Meckelian canal (=groove);

255 mx, maxilla; mx al, maxillary alveolus; n, nasal; occ con, occipital condyle; od, odontoid; orb,

256 orbit; os, osteoderm fragment; P1, first premaxillary alveolus; P2, second premaxillary alveolus;

257 P3, third premaxillary alveolus; ?p, possible parietal; p, parietal; ?pal, possible palatine; pal, 
258 palatine; pes, pes (foot); pmx, premaxilla; porb, postorbital; pop, paraoccipital process; prez,

259 prezygapophysis; prf, prefrontal; pt, pterygoid; pub b, pubic blade; $\mathbf{q}$, quadrate; $\mathbf{q j}$,

260 quadratojugal; rad, radius; retroart pr, retroarticular process; S? 1, possible first sacral vertebra;

261 S1, first sacral vertebra; S3, third sacral vertebra; spl, splenial; sq, squamosal; sub f, suborbital

262 fenestra; sup fen, supratemporal fenestra; supraac cr, supraacetabular crest; supraocc,

263 supraoccipital; suran, surangular; $\mathbf{t}$, isolated tooth; ?tib, possible tibia; tib, tibia; ul, ulna.

264

265 Methods

266

\subsection{Objectives and taxonomic sample}

267 Our phylogenetic analysis focused specifically on valid Teleosauroidea taxa, which range from 268 the Early Jurassic (lower Toarcian, e.g. Steneosaurus gracilirostris) to the Early Cretaceous 269 (Machimosaurus rex Fanti et al., 2016). The current dataset is a newly modified version of the $270 \mathrm{H}+\mathrm{Y}$ dataset. It has since grown substantially over the past three years, with the addition of new

271 taxa and characters. It was first presented in Ristevski et al. (2018) and has been updated

272 subsequently since then (Ösi et al. (2018); Foffa et al. (2019); Johnson, Young \& Brusatte

273 (2019); Sachs et al. (2019a, 2019b)).

274

Our taxonomic sample consisted of 153 crocodylomorph taxa (OTUs) with Postosuchus

275 kirkpatricki Chatterjee, 1985 as the outgroup taxon. Eighty OTUs are thalattosuchians, and 27 of

276 these are teleosauroids, listed as follows: 'Steneosaurus' gracilirostris; Mystriosaurus laurillardi

277 Kaup, 1834; 'Steneosaurus' stephani Hulke, 1877; the Chinese teleosauroid IVPP V 10098

278 previously referred to as Peipehsuchus teleorhinus (Li, 1993); Indosinosuchus potamosiamensis 
279 Martin et al., 2019; Indosinosuchus kalasinensis sp. nov. (see below); 'Steneosaurus' baroni;

280 Platysuchus multiscrobiculatus; Teleosaurus cadomensis; Mycterosuchus nasutus; Bathysuchus

281 megarhinus; 'Steneosaurus' bollensis; 'Steneosaurus' leedsi; Sericodon jugleri von Meyer,

282 1845; Aeolodon priscus; 'Steneosaurus' megistorhynchus Eudes-Deslongchamps, 1866a;

283 Yvridiosuchus boutilieri (Eudes-Deslongchamps, 1868b) Johnson, Young \& Brusatte, 2019;

284 Deslongchampsina larteti (Eudes-Deslongchamps, 1866a) Johnson, Young \& Brusatte, 2019;

285 'Steneosaurus' bouchardi Sauvage, 1872; 'Steneosaurus' heberti; Steneosaurus rostromajor

286 Geoffroy Saint-Hilaire, 1825; 'Steneosaurus' edwardsi; Lemmysuchus obtusidens;

287 Machimosaurus buffetauti; Machimosaurus mosae; Machimosaurus hugii; and Machimosaurus

288 rex. Certain taxa were excluded from the dataset, being either fragmentary, lost or correspondent

289 with known species (see discussion below). First-hand examination of all aforementioned

290 teleosauroid taxa (excluding 'S.' bouchardi and certain Ser. jugleri specimens) by MMJ resulted

291 in the modification of the dataset. The differences between this dataset and that provided in the

292 most recently updated $\mathrm{H}+\mathrm{Y}$ analysis (Johnson, Young \& Brusatte, 2019) are as follows:

293

294

295

296

297

298

299

300

1. Eight new taxa were added: 'S.' stephani, I. potamosiamensis, I. kalasinensis sp. nov., Ser. jugleri, 'S.' bouchardi, 'S.' baroni, 'S.' megistorhynchus and $S$. rostromajor.

2. Generic names were changed for three previously included taxa (Yvridiosuchus, Bathysuchus and Deslongchampsina).

3. Steneosaurus brevior was changed to Mystriosaurus laurillardi following Sachs et al. (2019a).

4. All characters of all remaining teleosauroid taxa were re-examined and re-scored.

Peer] reviewing PDF | (2020:03:47227:2:0:NEW 29 Jul 2020) 
301

302

303

304

305

306

307

308

309

310

311

312

313

314

315

316

317

318

319

320

321 (339 dental+craniomandibular, 120 post-cranial and 5 soft tissue); Sachs et al. (2019a) 322 incorporated 462 characters ( 337 dental + craniomandibular, 120 post-cranial and 5 soft tissue);

5. The number of characters increased from 464 to 502 (new characters $12,13,15,43$, $56,58,64,124,125,167,184,208,269,270,291,292,293,294,295,296,297,339$, $340,394,395,396,398,417,430,431,434,438,449,456,459,464,466$ and 489).

6. Characters 32 and 36 were re-written.

7. Character 27 was re-written and re-defined.

8. Characters 47 and 48 were re-written and re-scored, referring to characteristics of the pholidosaurid 'beak' (ch. 47) and teleosauroid premaxilla (ch. 48).

9. 19 additional characters were ordered $(49,57,85,101,107,178,179,203,241,256$, $257,309,410,408,414,447,452,457$ and 471).

10. Two non-teleosauroid taxa were excluded (Eoneustes bathonicus (Mercier, 1933) Young et al., 2010; and Geosaurine indeterminate from Argentina) and four were included (the early crocodylomorph Carnufex carolinensis Zanno et al., 2015; Metriorhynchoid indeterminate T; Maledictosuchus nuyivijanan Barrientos-Lara, Alvarado-Ortega \& Fernández, 2018; and Swiss 'Metriorhynchus hastifer').

\subsection{Character sampling and scoring}

The foundation of our character sampling is the $\mathrm{H}+\mathrm{Y}$ dataset, which initially included 387 characters (Ristevski et al., 2018), with 289 dental+craniomandibular, 95 post-cranial and 3 soft

tissue. Ősi et al. (2018) contained 454 characters (334 dental+craniomandibular, 116 post-cranial and 4 soft tissue); Foffa et al. (2019) incorporated 456 characters (336 dental+craniomandibular, 116 postcranial, and 4 soft tissue); Johnson, Young \& Brusatte (2019) included 464 characters 
323 and Sachs et al. (2019b) used 460 characters (337 dental+craniomandibular, 118 post-cranial and

3245 soft tissue).

325 In our updated version of the $\mathrm{H}+\mathrm{Y}$ dataset, 38 new characters were added (362

326 dental+craniomandibular, 135 post-cranial and 5 soft tissue). The complete character list

327 comprises of 502 characters, including 286 craniomandibular (57\%), 76 dental (15\%), 135 post-

328 cranial (27\%) and 5 soft tissue (1\%). Out of 502 characters, 45 were treated as ordered: 7, 26, 39,

$32947,49,59,62,71,85,101,107,112,178,179,181,183,193,203,224,241,242,250,256,257$,

$330282,301,309,359,385,388,397,408,409,410,414,447,450,452,453,457,467,468,470$,

331 471, and 482. The characters were scored based on first-hand examination of numerous

332 teleosauroid specimens. Additional, unavailable or lost specimens pertaining to Mac. hugii, Mac.

333 mosae and Sericodon were also examined from photographs (Hua (1999); Lepage et al. (2008);

334 Young et al. (2014); Schaefer, Püntener \& Billon-Bruyat (2018)), and photographs of 'S.'

335 bouchardi were provided by Y. Lepage. In addition, multiple Steneosaurus sp., Machimosaurus

336 sp., Teleosaurus sp. and Teleosauroidea indeterminate specimens were examined. Overall,

337 approximately 550 teleosauroid specimens were personally studied by MMJ.

The complete list of 502 characters are presented the Supplementary Material (SD1),

339 similar to Ösi et al. (2018), Foffa et al. (2019), Johnson, Young \& Brusatte (2019) and Sachs et

340 al. (2019a, 2019b). Newly added characters are represented by (NEW), ordered characters are

341 specified by (ORDERED), and characters that cannot be scored (e.g. are inapplicable) for all

342 taxa are marked with an asterisk (*) following the character descriptions. Additional comments

343 and references are included, and characters are organized in the following anatomical order:

344 1. Skull geometry and dimensions

Peer) reviewing PDF | (2020:03:47227:2:0:NEW 29 Jul 2020) 
345 2. Craniomandibular ornamentation

346 3. Internal neuroanatomy, sensory systems and cranial exocrine glands

347 4. Craniomandibular pneumaticity

348 5. Rostral neurovascular foramina

349 6. Cranial rostrum

350 7. Skull roof

351 8. Orbit and temporal region

352 9. Palate and perichoanal structures

353 10. Occipital

354 11. Braincase, basicranium and suspensorium

355 12. Mandibular geometry

356 13. Mandible

357 14. Dentition and alveolar morphologies

358 15. Axial post-cranial skeleton

359 16. Appendicular skeleton: pectoral girdle and forelimbs

360 17. Appendicular skeleton: pelvic girdle and hind limbs

361 18. Dermal ossifications: osteoderms

362 19. Dermal ossifications: gastralia

363 20. Soft tissue

364 1.3 Methodology

365 Our dataset, which includes 153 OTUs and 502 characters, was analysed by conducting 366 unweighted and weighted maximum parsimony analyses using TNT 1.5 Willi Hennig Society 
367 Edition (Goloboff et al., 2008; Goloboff and Catalano, 2016), following previous iterations (Ösi

368 et al., 2018; Foffa et al., 2019; Johnson, Young \& Brusatte, 2019; Sachs et al., 2019a, 2019b).

Our dataset was analysed as previously described in Foffa et al. (2019), Johnson, Young

370 \& Brusatte (2019), and Sachs et al. (2019a, 2019b). Specifically, memory settings were increased

371 with General RAM set to $900 \mathrm{Mb}$ and the maximum number of trees to be held set to 99,999 .

372 Cladogram space was searched by means of the 'New Technology search' option in TNT

373 (Sectorial Search, Ratchet, Drift, and Tree fusing) with 1000 random-addition replicates (RAS).

374 The trees were then subjected to a Traditional Search, with 'tree bisection reconnection' (TBR)

375 branch swapping, using 1000 replications and 10 trees saved per replication. In addition, the

376 default setting was increased for the iterations of each method (except for Tree fusing, which was

377 kept at three rounds). In the Sectorial Search, 1000 Drift cycles (for selections of above 75) were

378 run, as well as 1000 starts and fuse trees (for selections below 75) and 1000 rounds of Consensus

379 Sectorial Searches (CSSs) and Exclusive Sectorial Searches (XSSs). For Ratchet, the program

380 used 1000 ratchet iterations set to stop the perturbation when 1000 substitutions were made or

$38199 \%$ of the swapping was reached. Lastly, in Drift, the analysis included 1000 Drift cycles set to

382 stop the perturbation when 1000 substitutions were made or $99 \%$ of the swapping was reached.

383 The collapsing rule used was $50 \%$, and Bremer support values of 10 were also computed which

384 measure branch support and indicate the number of extra steps required for a clade to collapse

385 (Bremer, 1988; Müller, 2004). In addition, a majority rules unweighted consensus (50\% cut-off)

386 was examined, as it summarizes a specific collection of MPTs (Holder, Sukumaran \& Lewis,

387 2008). The analysis was run again using implied weighing $(\mathrm{k}=12)$, with the 'New Technology

388 search' options (Sectorial Search, Ratchet, Drift, and Tree fusing) with the same settings as

389 outlined above. 
391 v3.2.6 (Huelsenback \& Ronquist, 2001; Huelsenback et al., 2001; Ronquist et al., 2012). While

392 Bayesian methods are generally more popular when using molecular phylogenetics, they are

393 becoming more common in morphological studies, including those involving fossil data (e.g.

394 Lewis, 2001; Prieto-Márquez, 2010; Slater, 2013; Brusatte \& Carr, 2016). We chose to run our

395 dataset in MrBayes to compare its results with that of the unweighted and weighted topologies in

396 TNT. The Markov (Mk) model of Lewis (2001) was used, with three different variations applied.

397 The first was a generalized test, using the default setting of MrBayes: this is the simplest model,

398 in that all substitutions have the same rate or involves equal rates of character change

399 (rates=equal). The second involved a gamma parameter distribution with four rate categories

400 (rates=gamma ngammacat $=4$ ), which allows for differing rates of character change. The

401 rates $=$ gamma refers to gamma distribution rates across sites, and ngammacat sets the number of

402 rate categories for the gamma distribution. The third involves a slightly different gamma

403 parameter distribution (lset applyto=(1) coding=variable rates $=$ gamma $)$. This test specifies how

404 characters are sampled, with variable indicating that only variable characters have the possibility

405 of being sampled. In all three analyses, four chains were used and ran for 4,000,000 generations,

406 sampled every 100 generations. Trees that were generated during the first 20,000 generations

407 were disregarded as 'burn in'.

408

409 Systematic Palaeontology - Genus and species level taxonomy

410 As mentioned previously, the most historically important and commonly utilized teleosauroid

411 genus Steneosaurus has been recognized as a 'wastebasket' taxon by researchers and has 
412 continuously been recovered as paraphyletic or polyphyletic in phylogenetic analyses (e.g.

413 Mueller-Töwe, 2006; Wilberg, 2015b; Foffa et al., 2019; Johnson, Young \& Brusatte, 2019). In

414 addition, no type species had until recently been officially designated for Steneosaurus under

415 International Commission on Zoological Nomenclature (ICZN) Code rules. Johnson, Young \&

416 Brusatte (2020) set out to rectify this problem by evaluating the validity of Steneosaurus. The

417 authors designated Steneosaurus rostromajor Geoffroy Saint-Hilaire, 1825, as the type species of

418 Steneosaurus, designated MNHN.RJN 134c-d as the lectotype, provided a thorough literature

419 and descriptive review of the specimen, and compared it with other relevant teleosauroid taxa.

420 Their final verdict considered S. rostromajor (MNHN.RJN 134c-d) to be a nomen dubium, and

421 proposed that the genus Steneosaurus is undiagnostic, due to (1) lack of autapomorphic

422 characters (2) poor preservation (3) a generic concept that has changed multiple times through

423 time; and (4) uncertainty of teleosauroid ontogenetic variation and sexual dimorphism (Johnson,

424 Young \& Brusatte, 2020).

Johnson, Young \& Brusatte (2020) suggested that establishing a 'clean' foundation of

426 teleosauroid taxonomy using diagnostic type species/specimens, with every nomenclatural act

427 correctly formulated, was the next course of action. Therefore, we believe that it is necessary to

428 erect new proposed teleosauroid genera first, as a direct result of the proposal of Steneosaurus as

429 a nomen dubium.

This article in Portable Document Format (PDF) signifies a published work in accordance

431 with the ICZN. As such, the new genus and species names contained will be effectively

432 published under ICZN Code from the electronic edition. This work and the nomenclatural acts

433 contained within it have been registered in ZooBank, the online registration system for the ICZN. 
434 The following ZooBank LSIDs (Life Science Identifiers) and associated information may be

435 viewed through a standard web browser by adding the LSID to the prefix http://zoobank.org/.

436 The LSID for this publication is: urn:lsid:zoobank.org:pub:7CC3CA17-F08F-48AD-9F16-

437 8537B6BAAC1F.

438

439

CROCODYLOMORPHA Hay, 1930 (sensu Nesbitt 2011)

440

THALATTOSUCHIA Fraas, 1901 (sensu Young and Andrade 2009)

441

TELEOSAUROIDEA Geoffroy Saint-Hilaire, 1831 (sensu herein, see below)

442

Plagiophthalmosuchus gen. nov.

443 Type species-Steneosaurus gracilirostris Westphal, 1961. Now referred to as

444 Plagiophthalmosuchus gracilirostris (Westphal, 1961), comb. nov.

445 urn:lsid:zoobank.org:act:1AC91E3C-FC9A-470B-B9A9-3220B9823C0F

446 Etymology — 'Lateral-eyed crocodile.' Plágios ( $\pi \lambda \alpha \dot{\alpha} \gamma 10 \varsigma)$ and ofthalmós (o $\varphi \theta \alpha \lambda \mu o ́ \varsigma)$ are Greek

447 for 'lateral' and 'eye', respectively (referring to the laterally directed orbits of this taxon); suchus

448 is the Latinized form of the Greek soukhos ( $\sigma 0 \tilde{v} \chi 0 \varsigma)$, meaning crocodile.

449 Diagnosis - same as the only known species (monotypic genus).

450

Plagiophthalmosuchus gracilirostris (Westphal, 1961) comb. nov.

(Fig. 2) 
452 Holotype-NHMUK PV OR 14792, a nearly complete skeleton.

453 Paratype-NHMUK PV OR 15500, a complete skull and mandible.

454 Referred material-DONMG specimen (nearly complete skull and mandible); MNHNL TU515

455 (nearly complete skull and mandible); YORM 2012.38 (nearly complete skull).

456 Age - early Toarcian, Early Jurassic.

457 Localities - Whitby, Yorkshire, UK; Dudelange-Bettembourg, southern Luxembourg.

458 Stratigraphic horizons - Alum Shale Member, Whitby Mudstone Formation, Lias Group;

459 Harpoceras serpentinum ammonite Zone ('schistes bitumineux').

460 Scoring Sources - the holotype (NHMUK PV OR 14792), paratype and all referred specimens

461 were studied first-hand. Photographs of DONMG were provided by D. Lomax.

462 Autapomorphic characters of Pla. gracilirostris - in the antorbital fenestra, the external

463 fenestra is significantly larger than internal fenestra (over 25\%); antorbital fenestra is moderately

464 large, being at least half the diameter of the orbit; internal fenestra is approximately $50 \%$ of the

465 length of the orbit; supratemporal fossa is slightly larger $(\sim 25 \%)$ than the length of the orbit;

466 basioccipital sub-vertical and somewhat visible in occipital view; exoccipital-opisthotics are

467 dorsoventrally slender and paraoccipital processes have a straight distal margin; orbit positioned

468 laterally with a slight dorsal inclination; dorsal border at dentary-surangular is relatively straight;

469 glenoid fossa of the articular oriented subtly anterodorsally. 
470 Emended diagnosis - longirostrine snout; tooth row and quadrate condyle aligned, both at a

471 lower level than the occipital condyle (shared with Macrospondylus); ornamentation absent on

472 prefrontal (shared with I. potamosiamensis, Aeolodon, Bathysuchus and Sericodon) and lacrimal

473 (shared with I. potamosiamensis, Sericodon, Aeolodon and Macrospondylus); greater than 67\%

474 of the total premaxilla length is posterior to the external nares (similar to the Chinese

475 teleosauroid, I. potamosiamensis, Mycterosuchus, Aeolodon, Bathysuchus and Sericodon);

476 external nares oriented anterodorsally (shared with Indosinosuchus, the Chinese teleosauroid,

477 Teleosaurus, Platysuchus, Mycterosuchus, Aeolodon, Bathysuchus and Sericodon); premaxilla

478 anterior and anterolateral margins are not sub-vertical (shared with Macrospondylus,

479 Andrianavoay, Charitomenosuchus, Deslongchampsina, Proexochokefalos, Neosteneosaurus

480 and Machimosaurini); antorbital fenestra is anteroposteriorly elongated (similar to

481 Deslongchampsina); frontal broader than orbital width (shared with Mystriosaurus, Platysuchus,

482 Teleosaurus, Mycterosuchus, Bathysuchus, Aeolodon, Pr. cf. bouchardi, Neosteneosaurus, Mac.

483 buffetauti and Mac. mosae); squamosal projects further posteriorly than the occipital condyle

484 (shared with the Chinese teleosauroid, Neosteneosaurus, Yvridiosuchus, Lemmysuchus and Mac.

485 mosae); orbit longitudinal ellipsoid in shape; basioccipital tubera reduced (shared with

486 Mycterosuchus, Bathysuchus and Sericodon); supraoccipital dorsoventrally tall (shared with

487 Clovesuurdameredeor, Andrianavoay and Lemmysuchus); angular straight and mainly

488 horizontal, especially the anterior part (shared with Mystriosaurus); ventral margin of mandible

489 is poorly curved (shared with Mystriosaurus); proximal humerus expanded and hooked (similar

490 to Platysuchus and Teleosaurus); tibia evidently shorter than the femur (shared with

491 Platysuchus).

492

Peer) reviewing PDF | (2020:03:47227:2:0:NEW 29 Jul 2020) 
494 Type species-Mystriosaurus laurillardi Kaup, 1834.

495 Etymology - 'Spoon lizard'. Mystrio refers to the spoon-shaped anterior rostrum in dorsal view,

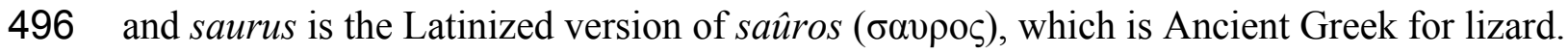

497 Diagnosis — same as the only known species (monotypic genus).

498 Mystriosaurus laurillardi Kaup, 1834

499

(Fig. 3)

500 Holotype-HLMD V946-948, a partial skull.

501 Referred material-NHMUK PV OR 14781 (nearly complete skull and mandible), holotype of 502 Steneosaurus brevior.

503 Age - Harpoceras serpentinum Sub-Boreal ammonite Zone, early Toarcian, Early Jurassic.

504 Localities-Altdorf, Germany; Whitby, Yorkshire, UK.

505 Stratigraphic horizons - Posidonia Shale Formation; Mulgrave Shale Member, Whitby 506 Mudstone Formation, Lias Group.

507 Scoring sources - NHMUK PV OR 14781 was studied first-hand. The holotype (HLMD V946508 948) was examined using high quality photographs provided by S. Sachs, and also discussed at 509 great length with S. Sachs. 
510 Autapomorphic characters of Mys. laurillardi - well-developed and extensive ornamentation

511 on the nasals; external nares oriented anteriorly; antorbital fenestra is sub-rectangular in shape;

512 supratemporal fossae form an approximate isosceles trapezoid-shape; medial margin of

513 supratemporal arch relatively straight in dorsal view, with no significant concavity; prominent

514 anterior notch in the dentaries; mandibular fenestra poorly elliptic; large robust teeth with

515 numerous, conspicuous apicobasally aligned enamel ridges and a pointed apex, with more

516 anteriorly-placed tooth crowns being procumbent.

517 Emended diagnosis - mesorostrine skull; well-developed and extensive ornamentation on the

518 premaxillae, maxillae, frontal, prefrontal, lacrimal and postorbital; frontal ornamentation

519 composed of small sub-circular to elongate pits that are closely spaced or, that can fuse and

520 become a ridge-groove pattern (similar to Mycterosuchus); slight constriction of the snout

521 anterior to the orbits (similar to Deslongchampsina); large and numerous neurovascular foramina

522 on the premaxillae, maxillae and dentaries (shared with Machimosaurini); external nares 8-

523 shaped in dorsal view (shared with the Chinese teleosauroid, I. potamosiamensis, Bathysuchus

524 and Aeolodon); dorsoventrally deep premaxilla (similar to I. kalasinensis); anteroposterior

525 premaxilla length less than $25 \%$ of total rostral length (shared with the Chinese teleosauroid,

526 Mac. buffetauti and Mac. mosae); premaxilla anterior and anterolateral margins are orientated

527 anteroventrally and extend ventrally in lateral view (shared with the Chinese teleosauroid,

528 Indosinosuchus, Platysuchus, Mycterosuchus, Aeolodon, Bathysuchus and Sericodon); antorbital

529 fenestrae almost equidistant to orbit and alveolar margin (shared with Platysuchus); antorbital

530 fenestra is large relative to orbits, where the anteroposterior length is approximately $25 \%$ orbital

531 anteroposterior length (similar to Plagiophthalmosuchus and Deslongchampsina); anterolateral

532 margin of supratemporal fossae noticeably inclined anterolaterally (shared with the Chinese 
533 teleosauroid, Indosinosuchus, Platysuchus, Teleosaurus, Mycterosuchus, Aeolodon, Bathysuchus

534 and Sericodon); the anterior region of the supratemporal fenestra has well-rounded lateral and

535 medial margins; frontal width broader than orbital width (shared with Plagiophthalmosuchus,

536 Platysuchus, Teleosaurus, Mycterosuchus, Aeolodon, Bathysuchus, Sericodon, Pr. cf. bouchardi,

537 Neosteneosaurus, Mac. buffetauti and Mac. mosae); very short frontal anteromedial process,

538 (similar to Clovesuurdameredeor); orbits subcircular in shape and dorsolaterally orientated;

539 postorbital reaches orbit posteroventral margin (shared with the Chinese teleosauroid, I.

540 potamosiamensis, Platysuchus, Teleosaurus and Mycterosuchus); mandibular symphysis slightly

541 less than half the mandibular length, between 45 and 50\% (shared with I. potamosiamensis,

542 Deslongchampsina and Proexochokefalos); deep, well-developed reception pits throughout the

543 anterior- to mid-maxilla and gradually disappear (similar to Charitomenosuchus,

544 Deslongchampsina and Proexochokefalos); ventral border of angular horizontal and poorly

545 curved, especially the anterior part (shared with Plagiophthalmosuchus); four teeth per

546 premaxilla; maxillary alveolar count at least 29 (modified from Young \& Steel, in press) (similar

547 to the Chinese teleosauroid, I. potamosiamensis, Neosteneosaurus, Yvridiosuchus and Mac.

548 buffetauti); dentary alveolar count approximately 30 to 33 alveolar pairs; P1 and P2 both oriented

549 anteriorly (shared with I. potamosiamensis, Platysuchus, Macrospondylus, Deslongchampsina,

550 Neosteneosaurus, Yvridiosuchus and Lemmysuchus).

551

552

Clovesuurdameredeor gen. nov. 
553 Type species-Steneosaurus stephani Hulke, 1877. Now referred to as Clovesuurdameredeor

554 stephani (Hulke, 1877), comb. nov. urn:lsid:zoobank.org:act:B9FC0E91-9153-4F6B-B4B7-

555 817839A9E7DD

556 Etymology - 'Clovesuurda's sea creature'. Clovesuurda was the Medieval Latin name of the

557 village of Closworth (written in the Doomsday Book of 1086), the locality where the holotype

558 was found; meredēor is Old English for 'sea creature'.

559 Diagnosis - same as the only known species (monotypic genus).

560

561

Clovesuurdameredeor stephani (Hulke, 1877) comb. nov.

562

(Fig. 4)

563 Holotype - NHMUK PV OR 49126, a partial skull and anterior section of mandible.

564 Age—Bathonian, Middle Jurassic.

565 Locality-Closworth, Dorsetshire, UK.

566 Stratigraphic horizon - Great Oolite Group, Cornbrash Formation.

567 Scoring sources - the holotype (NHMUK PV OR 49126) was examined first-hand.

568 Autapomorphic characters of $\boldsymbol{C l}$. stephani-prefrontal is anteroposteriorly short and

569 mediolaterally broadened; posterior projections of the nasals not elongated and level with

570 prefrontal-orbit contact in dorsal view; anteromedial process of the frontal is posterior to the 
571 prefrontals; anteromedial process of the frontal is anteroposteriorly short and mediolaterally

572 broad; jugal extends anteriorly to the prefrontal.

573 Emended diagnosis - frontal ornamentation extends from the centre to the lateral- and anterior-

574 most areas (shared with Plagiophthalmosuchus, the Chinese teleosauroid, Indosinosuchus,

575 Platysuchus, Teleosaurus, Mycterosuchus and Macrospondylus); presence of small antorbital

576 fenestrae; no anterolateral expansion or inclination of the supratemporal fenestrae (shared with

577 Plagiophthalmosuchus, Macrospondylus, Charitomenosuchus, Seldsienean, Deslongchampsina,

578 Proexochokefalos, Neosteneosaurus and Machimosaurini); frontal subequal to orbital width

579 (shared with the Chinese teleosauroid, I. kalasinensis, Macrospondylus, Charitomenosuchus,

580 Deslongchampsina, Proexochokefalos, Yvridiosuchus, Mac. hugii and Mac. rex); circular orbits

581 (shared with Mystriosaurus, Indosinosuchus, Teleosaurus, Mycterosuchus, Sericodon,

582 Lemmysuchus and Machimosaurus); anterior process of the jugal is slender and elongated

583 (shared with Charitomenosuchus, Proexochokefalos, Neosteneosaurus and Machimosaurini).

584

585 The Chinese teleosauroid previously referred to Peipehsuchus teleorhinus Young, 1948 (Li, 586

587

(Fig. 5)

588 Specimen-IVPP V 10098, a complete skull.

589 Age - Toarcian, Early Jurassic.

590 Locality—Daxian, Szechuan, China. 
591 Stratigraphic horizon-Ziliujing Formation.

592 Scoring sources-IVPP V 10098 was examined first-hand and was also discussed in great

593 length with E. Wilberg.

594 Autapomorphic characters of IVPP V 10098 - extreme constriction of premaxillae posterior to

595 external nares (relative to other teleosauroids), creating a laterally expanded, 'beak-like'

596 premaxilla; anterior- to mid-maxilla undulates mediolaterally in dorsal view; well-developed

597 palatal canals; the first premaxillary alveolus (P1) and second premaxillary alveolus (P2)

598 oriented immediately laterally to one another, with the anterior-most margins of both alveoli

599 sloping weakly anterolaterally; weak lateral expansion of the premaxilla (the P3 is situated

600 marginally ventrally to the P2); P3 is enlarged relative to the P2 and approximately the same size

601 as the P4.

602 Emended diagnosis - mesorostrine skull; tooth row and occipital condyle aligned, and quadrate

603 condyle at a lower level (shared with Charitomenosuchus, Proexochokefalos, Neosteneosaurus

604 and Machimosaurini); tooth row and occipital condyle aligned on the same plane with quadrate

605 at a slightly lower level (similar to Charitomenosuchus, Proexochokefalos, Neosteneosaurus and

606 Machimosaurini); shallow ornamentation of the premaxillae and maxillae (similar to

607 Indosinosuchus, Aeolodon, Bathysuchus and Sericodon); frontal ornamentation extends from the

608 centre to the lateral- and anterior-most areas (shared with Plagiophthalmosuchus,

609 Indosinosuchus, Platysuchus, Teleosaurus, Mycterosuchus, Macrospondylus and

610 Clovesuurdameredeor); external nares oriented anterodorsally (shared with

611 Plagiophthalmosuchus, Indosinosuchus, Platysuchus, Teleosaurus, Mycterosuchus, Aeolodon,

612 Bathysuchus and Sericodon); external nares '8-shaped' in anterior view (shared with 
613 Mystriosaurus, I. potamosiamensis, Bathysuchus and Aeolodon); premaxilla anteroposterior

614 length less than 25\% of total rostrum length (shared with Mystriosaurus, Mac. buffetauti and

615 Mac. mosae); premaxilla anterior and anterolateral margins are orientated anteroventrally and

616 extend ventrally (shared with Indosinosuchus, Platysuchus, Mycterosuchus, Aeolodon,

617 Bathysuchus and Sericodon); over $67 \%$ of total premaxilla length posterior to the external nares

618 (shared with Plagiophthalmosuchus, I. potamosiamensis, Mycterosuchus, Aeolodon,

619 Bathysuchus and Sericodon); small antorbital fenestrae present; supratemporal fenestrae sub-

620 rectangular in shape; anterolateral margin of supratemporal fossae noticeably inclined

621 anterolaterally (shared with Mystriosaurus, Indosinosuchus, Teleosaurus, Platysuchus,

622 Mycterosuchus, Aeolodon, Bathysuchus and Sericodon); frontal width subequal with orbital

623 width (shared with I. kalasinensis, Macrospondylus, Clovesuurdameredeor, Charitomenosuchus,

624 Proexochokefalos, Yvridiosuchus, Mac. hugii and Mac. rex); squamosal project further

625 posteriorly than occipital condyle (shared with Plagiophthalmosuchus, Neosteneosaurus,

626 Yvridiosuchus, Lemmysuchus and Mac. mosae); orbit anteroposteriorly elongated and ellipsoid in

627 shape (similar to Plagiophthalmosuchus, Platysuchus, Aeolodon, Macrospondylus,

628 Charitomenosuchus, Seldsienean, Deslongchampsina, Proexochokefalos and Neosteneosaurus);

629 postorbital reaches the orbit posteroventral margin (shared with Mystriosaurus, I.

630 potamosiamensis, Platysuchus, Teleosaurus and Mycterosuchus); pterygoid flange oriented

631 horizontally (shared with Teleosaurus); four premaxillary alveolar pairs; 27 maxillary alveolar

632 pairs; P3 and P4 do not form a couple (shared with Bathysuchus); small P1 compared to the P2

633 (similar to Macrospondylus).

634 Remarks - this taxon, along with the holotype of Peipehsuchus teleorhinus (IVPP RV 48001), is

635 currently being re-described by MM Johnson and colleagues.

Peer) reviewing PDF | (2020:03:47227:2:0:NEW 29 Jul 2020) 
636

637

Platysuchus Westphal, 1961

638 Type species-Mystriosaurus multiscrobiculatus Berckhemer, 1929. Now referred to as

639 Platysuchus multiscrobiculatus (Berckhemer, 1929), Westphal, 1961.

640 Etymology - 'Wide crocodile'. Platys comes from the Greek platýs ( $\pi \lambda \alpha \tau u ́ \varsigma)$ meaning wide

641 (referring to the flattened, expanded osteoderms and dermal shield), and suchus is the Latinized

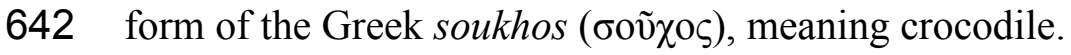

643 Diagnosis - same as the only known species (monotypic genus).

644

645

Platysuchus multiscrobiculatus (Berckhemer, 1929) Westphal, 1961

646

(Fig. 6)

647 Holotype-SMNS 9930, a nearly complete skeleton.

648 Referred material—MNHNL TU895 (a partial rostrum); UH 1 (complete skeleton).

649 Age —-lower Toarcian, Early Jurassic.

650 Localities-Holzmaden, Baden-Württemberg, Germany; Foetz, Luxembourg.

651 Stratigraphic horizons-Posidonia Shale Formation; Harpoceras serpentinum ammonite Zone 652 ('schistes bitumineux'). 
653 Scoring sources - the holotype (SMNS 9930) and MNHNL TU895 were examined first-hand.

654 Additional information was taken from Westphal (1961, 1962).

655 Autapomorphic characters of $\boldsymbol{P l}$. multiscrobiculatus - prefrontal and lacrimal both

656 ornamented with meandering, elongated grooves; mid- and posterior squamosal well ornamented

657 with small, circular, closely packed pits; frontal contribution to the intertemporal bar frontal

658 wider than the parietal in dorsal view; jugal excluded from the orbit by lacrimal-postorbital

659 contact; P1 and P2 do not form a couplet and are not oriented on the anterior margin of the

660 premaxilla; tuberculum of the dorsal rib medium-sized; ischium with thickened, robust ischial

661 neck; shortened, stocky pubis with a relatively subcircular proximal rim.

662 Emended diagnosis - longirostrine snout; tooth row and quadrate condyle unaligned with the

663 tooth row at a lower level, and both below the occipital condyle (shared with Teleosaurus); tooth

664 row at a lower level than the quadrate (shared with Plagiophthalmosuchus, Indosinosuchus,

665 Teleosaurus, Mycterosuchus and Macrospondylus); frontal ornamentation extends from the

666 centre to lateral- and anterior-most regions (shared with Plagiophthalmosuchus, the Chinese

667 teleosauroid, Indosinosuchus, Teleosaurus, Mycterosuchus, Macrospondylus and

668 Clovesuurdameredeor); external nares oriented anterodorsally (shared with

669 Plagiophthalmosuchus, the Chinese teleosauroid, Indosinosuchus, Teleosaurus, Mycterosuchus,

670 Aeolodon, Bathysuchus and Sericodon); the premaxilla anterior and anterolateral margins are

671 orientated anteroventrally and extend ventrally (shared with Mystriosaurus, the Chinese

672 teleosauroid, Indosinosuchus, Teleosaurus, Mycterosuchus, Aeolodon, Bathysuchus and

673 Sericodon); presence of small, mediolaterally thin antorbital fenestrae; anterior margin of the

674 supratemporal fossae are noticeably inclined anterolaterally (shared with Mystriosaurus, the 
675 Chinese teleosauroid, Indosinosuchus, Teleosaurus, Mycterosuchus, Aeolodon, Bathysuchus and

676 Sericodon); frontal width is broader than orbital width (shared with Plagiophthalmosuchus,

677 Mystriosaurus, Teleosaurus, Mycterosuchus, Bathysuchus, Aeolodon, Pr. cf. bouchardi,

678 Neosteneosaurus, Mac. buffetauti and Mac. mosae); frontal-postorbital suture is lower than the

679 intertemporal bar (shared with Teleosaurus); orbits are longitudinal ellipsoid in shape (shared

680 with Plagiophthalmosuchus, the Chinese teleosauroid, Aeolodon, Macrospondylus,

681 Charitomenosuchus, Seldsienean, Proexochokefalos, Deslongchampsina and Neosteneosaurus);

682 postorbital reaches the orbit posteroventral margin and forms an extensive area of the orbit

683 ventral margin (shared with Mystriosaurus, Indosinosuchus, the Chinese teleosauroid,

684 Teleosaurus and Mycterosuchus); five premaxillary alveoli (shared with Teleosaurus,

685 Bathysuchus and Sericodon); interalveolar spacing between P1-P2 and P3-P4 relatively the same

686 size (shared with Mycterosuchus, Bathysuchus and Sericodon); anterior maxillary teeth

687 procumbent (shared with Plagiophthalmosuchus, I. kalasinensis, Teleosaurus, Sericodon,

688 Aeolodon, Macrospondylus and Charitomenosuchus); neural spine height is greater than centrum

689 height (similar to Neosteneosaurus); tuberculum of dorsal rib situated on the medial edge (shared

690 with Aeolodon, Macrospondylus and Lemmysuchus); shortened and squat scapula (similar to

691 Macrospondylus); proximal humerus posteriorly expanded and weakly hooked (shared with

692 Teleosaurus); forelimb relatively shorter than hindlimb by approximately $22 \%$ (similar to

693 Macrospondylus); tibia shorter than the femur by approximately $25 \%$ (similar to

694 Macrospondylus); small round to ellipsoid pits on all osteoderms that are very densely

695 distributed, with a 'honeycomb' pattern (shared with Teleosaurus); presacral osteoderms are

696 strongly curved and closely locked together, forming a dorsal 'shield' (shared with Teleosaurus). 
698 Teleosaurus Geoffroy Saint-Hilaire, 1825

699 Type species-Crocodilus cadomensis Lamouroux, 1820. Now referred to as Teleosaurus 700 cadomensis (Lamouroux, 1820), Geoffroy Saint-Hilaire, 1825.

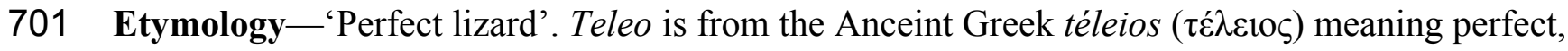

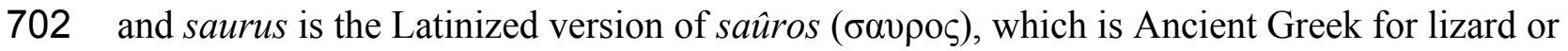
703 reptile.

704

705 Teleosaurus cadomensis Lamouroux, 1820

706 (Fig. 7)

707 Holotype-MNHN.F AC 8746, a partially complete skull, with associated postcranial material. 708 The specimen was initially found by Pierre Tesson, who traded it to Lamouroux. Lamouroux 709 briefly noted it (1820) and then sent the specimen to Georges Cuvier. It was fully described by 710 Cuvier (1824) and Geoffroy Saint-Hilaire (1825). See Brignon (2018a) for more details.

711 Referred material—NHMUK PV OR 119a (dorsal osteoderms); NHMUK PV R 4207 (dorsal 712 osteoderms); NHMUK PV OR 32588 (dorsal, sacral and caudal vertebrae); NHMUK PV OR 71332657 (femur); NHMUK PV OR 32680 (ischium); NHMUK PV OR 33124 (mandibular 714 symphysis); NHMUK PV OR 39788 (partial rostrum); and additional casts (e.g. NHMUK PV R 715 880; NHMUK PV R 880a).

716 Age-Bathonian, Middle Jurassic. 
717 Locality_Allemagne, $3 \mathrm{~km}$ south of Caen, Calvados, Normandy, France.

718 Stratigraphic horizon - 'Calcaire de Caen'.

719 Scoring sources - the neotype and all referred material mentioned above was studied first-hand.

720 Lamouroux (1820), Geoffroy Saint-Hilaire (1825), Eudes-Deslongchamps (1867-69), Vignaud

721 (1995) and Jouve (2009) provided additional information.

722 Autapomorphic characters of $T$. cadomensis - small, subcircular, shallow antorbital fenestrae;

723 supratemporal fenestrae box- or square-shaped; postorbital and squamosal are relatively the same

724 length, with the squamosal being slightly longer $(\sim 10 \%)$; choanae mediolaterally wider than

725 palatines.

726 Emended diagnosis - longirostrine, gracile snout; tooth row and quadrate condyle unaligned

727 with the tooth row at a lower level, and both below the occipital condyle (shared with

728 Platysuchus); tooth row at a lower level than the quadrate (shared with Plagiophthalmosuchus,

729 Indosinosuchus, Platysuchus, Mycterosuchus and Macrospondylus); rostrum narrows

730 immediately anterior to the orbits (shared with I. potamosiamensis, Mycterosuchus, Aeolodon,

731 Bathysuchus, Sericodon and Seldsienean); frontal ornamentation extends from the centre to

732 lateral- and anterior-most regions (shared with Plagiophthalmosuchus, the Chinese teleosauroid,

733 Indosinosuchus, Platysuchus, Mycterosuchus, Macrospondylus and Clovesuurdameredeor);

734 external nares oriented anterodorsally (shared with Plagiophthalmosuchus, the Chinese

735 teleosauroid, Indosinosuchus, Platysuchus, Mycterosuchus, Aeolodon, Bathysuchus and

736 Sericodon); premaxilla anterior and anterolateral margins of are orientated anteroventrally and

737 extend ventrally (shared with Mystriosaurus, the Chinese teleosauroid, Indosinosuchus, 
738 Platysuchus, Mycterosuchus, Aeolodon, Bathysuchus and Sericodon); anterior margin of the

739 supratemporal fossae are noticeably inclined anterolaterally (shared with Mystriosaurus, the

740 Chinese teleosauroid, Indosinosuchus, Platysuchus, Mycterosuchus, Bathysuchus and Aeolodon);

741 anteromedial projection of the frontal is relatively broad but becomes instantly mediolaterally

742 thin at the anterior-most part (shared with Sericodon); frontal width is broader than orbital width

743 (shared with Plagiophthalmosuchus, Mystriosaurus, Platysuchus, Mycterosuchus, Bathysuchus,

744 Aeolodon, Pr. cf. bouchardi, Neosteneosaurus, Mac. buffetauti and Mac. mosae); frontal-

745 postorbital suture is lower than the intertemporal bar (shared with Platysuchus); dorsal margins

746 of orbits upturned (shared with I. potamosiamensis, Mycterosuchus and Aeolodon); postorbital

747 reaches the orbit posteroventral margin and forms an extensive area of the orbit ventral margin

748 (shared with Mystriosaurus, Indosinosuchus, the Chinese teleosauroid, Platysuchus and

749 Mycterosuchus); pterygoid flange oriented horizontally (shared with the Chinese teleosauroid);

750 five premaxillary alveolar pairs (shared with Platysuchus, Bathysuchus and Sericodon); anterior

751 maxillary teeth procumbent (shared with Indosinosuchus, Platysuchus, Aeolodon, Sericodon,

752 Macrospondylus and Charitomenosuchus); proximal humerus posteriorly expanded and weakly

753 hooked (shared with Platysuchus); small round to ellipsoid pits that are very densely distributed,

754 with a 'honeycomb' pattern (shared with Platysuchus); presacral osteoderms are strongly curved

755 and closely locked together, forming a dorsal 'shield' (shared with Platysuchus).

756 Remarks - the genus Teleosaurus, initially defined by Geoffroy Saint-Hilaire (1825), has

757 encompassed numerous species throughout its long history, such as T. gladius, T. subulidens, $T$.

758 geoffroyi, T. minimus and T. eucephalus (Quenstedt, 1852; Phillips, 1871; Seeley, 1880; Eudes-

759 Deslongchamps, 1868c). However, the majority of these historic Teleosaurus species are

760 currently considered invalid due to the following propositions: (1) thought to be juveniles or sub- 
761 adults, and therefore subjective junior synonyms of T. cadomensis (e.g. Jouve, 2009); (2)

762 uncertainty of teleosauroid ontogenetic stages and sexual dimorphism (see Johnson, Young \&

763 Brusatte, 2020); and (3) loss of original material. Therefore, we currently only recognize $T$.

764 cadomensis as a valid species; the issue regarding the validity of other 'Teleosaurus' species is

765 beyond the scope of this manuscript.

766

767 Mycterosuchus Andrews, 1913

768 Type species_-Steneosaurus nasutus Andrews, 1909. Now referred to as Mycterosuchus nasutus 769 (Andrews, 1909), Andrews, 1913.

770 Etymology - '[Long] Nose crocodile'. Myctero comes from the Latin mycto meaning nose,

771 referring to the elongated rostrum of this taxon; suchus is the Latinized form of the Greek

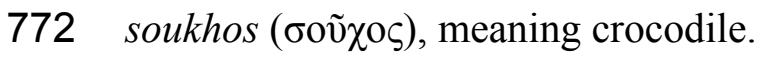

773 Diagnosis — same as the only known species (monotypic genus).

774

775 Mycterosuchus nasutus (Andrews, 1909) Andrews, 1913

776 (Fig. 8)

777 Holotype - NHMUK PV R 2167, a complete skull and mandible, with additional material

778 (including vertebrae [cervical, dorsal, sacral and caudal], cervical and dorsal ribs, 
779 scapulocoracoid, two partial femora, one radius, one ulna, multiple phalanges and tarsals,

780 isolated teeth and multiple dorsal osteoderms).

781 Referred material—CAMSM J.1420 (nearly complete skeleton); NHMUK PV R 3892 (dorsal

782 and sacral vertebrae); NHMUK PV R 4059 (partial skull); unnumbered GZG specimen

783 (complete skull). Possible NM partial skeleton (catalogue number unknown, photographs

784 provided by B. Ekrt).

785 Age $—$ Middle Callovian, Middle Jurassic.

786 Locality—Peterborough, UK.

787 Stratigraphic horizon-Peterborough Member, Oxford Clay Formation, Ancholme Group.

788 Scoring sources - the holotype (NHMUK PV R 2167) and all referred material (excluding the

789 NM skeleton) mentioned above were studied first-hand.

790 Autapomorphic characters of Myc. nasutus — overall cranium and mandible extremely rugose;

791 elongate, slender rostrum (approximately $73 \%$ of total skull length); maxilla ornamented with an

792 array of irregular patterns of deep rugosities and anastomosing grooves; reduced quadrate

793 condyles; palatine anterior margin terminates level to 29th maxillary alveoli, or more distal

794 alveoli; curvature of the angular is gradual in the anterior region, but more abrupt in the

795 posterior-most region; on the retroarticular process, the length of the attachment surface for the

796 adductor muscles is more than twice its width; D1 strongly anteriorly oriented; the neural arches

797 of the posterior cervical vertebrae are taller than the vertebral centra; the posterior edge of the

798 scapula is more strongly concave than the anterior edge; the humeral head is weakly posteriorly 
799 expanded and hooked with a club-like shape; the ulna is more than $25 \%$ longer than the radius;

800 the pubic shaft is over 50\% length of the pubic plate; anteromedial tuber of the femur is the

801 largest of the proximal tubera; size of calcaneal tuber approximately $25 \%$ of total astragalus size;

802 large, heavyset dorsal osteoderms with large, round-to-ellipsoid (D-shaped) irregular pits that are 803 well separated from one another.

804 Emended diagnosis - longirostrine snout; tooth row and quadrate condyle unaligned and 805 quadrate at a lower level, but both below the occipital condyle (shared with Indosinosuchus 806 taxa); well-developed and extensive ornamentation on the premaxillae, maxillae, frontal, 807 prefrontal, lacrimal and postorbital; frontal ornamentation composed of small sub-circular to 808 elongate pits that are closely spaced or, that can fuse and become a ridge-groove pattern (similar 809 to Mystriosaurus); rostrum narrows immediately anterior to the orbits (shared with $I$.

810 potamosiamensis, Teleosaurus, Aeolodon, Bathysuchus, Sericodon and Seldsienean); premaxilla 811 anterior and anterolateral margins are strongly anteroventrally deflected and extend ventrally 812 (shared with Mystriosaurus, the Chinese teleosauroid, Indosinosuchus, Platysuchus,

813 Teleosaurus, Aeolodon, Bathysuchus and Sericodon); more than 67\% of total premaxilla length 814 is posterior to the external nares (shared with Plagiophthalmosuchus, I. potamosiamensis, the 815 Chinese teleosauroid, Aeolodon, Bathysuchus and Sericodon); external nares are '8' shaped in 816 dorsal view due to enlarged anterior and posterior projections of the premaxilla (shared with

817 Bathysuchus); external nares are anterodorsally oriented (shared with Mystriosaurus, the Chinese 818 teleosauroid, Platysuchus and Bathysuchus); clustering of large, circular foramina along lateral 819 margin of external nares (similar to Mystriosaurus, I. kalasinensis and Machimosaurini); small, 820 subcircular antorbital fenestrae; the anterior margin of the supratemporal fossae are noticeably 821 inclined anterolaterally (shared with Mystriosaurus, the Chinese teleosauroid, Indosinosuchus, 
822 Platysuchus, Teleosaurus, Aeolodon, Bathysuchus and Sericodon); frontal width broader than

823 orbital width (shared with Plagiophthalmosuchus, Mystriosaurus, Platysuchus, Teleosaurus,

824 Bathysuchus, Aeolodon, Neosteneosaurus, Mac. buffetauti and Mac. mosae); circular orbits

825 (shared with Mystriosaurus, Teleosaurus, Indosinosuchus, Clovesuurdameredeor and

826 Machimosaurini); dorsal margins of orbits are upturned (shared with I. potamosiamensis,

827 Teleosaurus and Aeolodon); postorbital reaches the orbit posteroventral margin and extensively

828 forms part of the orbit ventral margin (shared with Mystriosaurus, the Chinese teleosauroid, $I$.

829 potamosiamensis, Platysuchus and Teleosaurus); reduced basioccipital tubera (similar to

830 Plagiophthalmosuchus, Bathysuchus and Sericodon); mandibular symphysis over $50 \%$ of

831 mandible length (shared with Bathysuchus, Aeolodon, Macrospondylus, Seldsienean and

832 Charitomenosuchus); mandibular symphysis depth is very narrow, approximately $4-4.5 \%$ of the

833 mandible length (shared with Charitomenosuchus); the P1 and P2 do not form a couplet, and the

834 interalveolar spacing between the P1-P2 and P3-P4 are relatively the same size (shared with

835 Platysuchus, Bathysuchus and Sericodon); both the P1 and P2 alveoli are oriented laterally

836 (shared with Bathysuchus and Sericodon); the P1 and P2 do not form a couplet but are still

837 oriented on the anterior margin of the premaxilla (shared with Bathysuchus and Sericodon); P1

838 and P2 are on the same transvers plane (shared with Aeolodon, Bathysuchus and Sericodon);

839 teeth slender, pointed and weekly mediolaterally compressed (shared with Bathysuchus and

840 Aeolodon); the tubercula and articular facets in the dorsal ribs are positioned directly in the

841 middle (shared with Charitomenosuchus); the tubercula in the dorsal ribs are large and

842 pronounced (shared with Neosteneosaurus and Machimosaurini); tibia approximately 40-50\%

843 shorter than the femur (shared with Charitomenosuchus, Neosteneosaurus and Machimosaurini); 
844 the medial femoral condyle is noticeably larger than the lateral femoral condyle (shared with

845 Charitomenosuchus and Neosteneosaurus).

846 Remarks - the skull and mandible of the NHMUK holotype was originally numbered PV R

8472617 , along with the associated postcranial material. The skull and mandible were then

848 reregistered PV R 3577 in error (what year and by whom is unknown). Mycterosuchus has also

849 been considered as a synonym of Steneosaurus leedsi (= Charitomenosuchus leedsi) in certain

850 studies (e.g. Vignaud, 1995).

851

852

Aeolodon von Meyer, 1832

853 Type species-Crocodilus priscus von Sömmerring, 1814. Now referred to as Aeolodon priscus

854 (von Sömmerring, 1814), von Meyer, 1832.

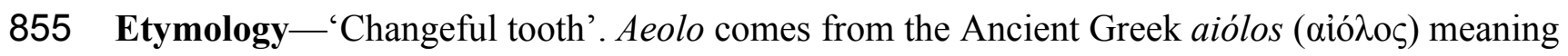

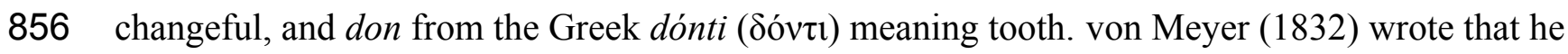

857 used this name based on the holotype's "heterodont teeth".

858 Diagnosis - same as the only known species (monotypic genus).

859

860

Aeolodon priscus (von Sömmerring, 1814) von Meyer, 1832

861

(Fig. 9) 
862 Holotype-NMHUK PV R 1086, a nearly complete skeleton.

863 Referred material-MNHN.F.CNJ 78 (nearly complete skeleton).

864 Age-Lower Tithonian, Late Jurassic.

865 Localities - Daiting, southern Germany; Canjuers, Var, France.

866 Stratigraphic horizons-Mörnsheim Formation; Canjuers conservation Lagerstätte.

867 Scoring sources - the holotype (NMHUK PV R 1086) and referred specimen (MNHN.F.CNJ

868 78a) were both studied first-hand.

869 Autapomorphic characters of $\boldsymbol{A}$. priscus - shallow elliptical pits on the frontal; length of the

870 attachment surface for the m. pterygoideus posterior on the retroarticular process is short, and

871 subequal to its width; neural spine and centrum heights of the mid-cervical vertebrae are

872 approximately equal; distal coracoid with rounded edges and a deep coracoid foramen; extremely

873 shortened ulna and radius relative to humerus; ulna with little curvature, only in the proximal-

874 most region; metacarpals IV and V are similar in robusticity to II-III ; ischial plate sub-

875 triangular; tibia 30-40\% shorter than the femur; dorsal osteoderm ornamentation consists of

876 large, well-spaced circular pits.

877 Emended diagnosis - longirostrine skull; rostrum narrows immediately anterior to the orbits

878 (shared with I. potamosiamensis, Teleosaurus, Mycterosuchus, Bathysuchus, Sericodon and

879 Seldsienean); shallow, inconspicuous ornamentation of the premaxillae and maxillae (similar to

880 the Chinese teleosauroid, Indosinosuchus, Bathysuchus and Sericodon); no ornamentation on the

881 prefrontal (shared with Plagiophthalmosuchus, I. potamosiamensis, Bathysuchus and Sericodon) 
882 and lacrimal (shared with Plagiophthalmosuchus, I. potamosiamensis, Sericodon,

883 Macrospondylus and Charitomenosuchus); frontal ornamentation restricted to centre (shared

884 with Sericodon, Charitomenosuchus, Seldsienean, Deslongchampsina, Proexochokefalos,

885 Neosteneosaurus and Machimosaurini); external nares oriented anterodorsally (shared with the

886 Chinese teleosauroid, Indosinosuchus, Platysuchus, Teleosaurus, Mycterosuchus, Bathysuchus

887 and Sericodon); external nares noticeably ' 8 '-shaped in anterior view (shared with

888 Mystriosaurus, the Chinese teleosauroid, I. potamosiamensis and Bathysuchus); the premaxilla

889 anterior and anterolateral margins are orientated anteroventrally and extend ventrally (shared

890 with Mystriosaurus, the Chinese teleosauroid, Indosinosuchus, Platysuchus, Teleosaurus,

891 Mycterosuchus, Bathysuchus and Sericodon); sub-rectangular supratemporal fenestrae; the

892 anterior margin of the supratemporal fossae are noticeably inclined anterolaterally (shared with

893 Mystriosaurus, the Chinese teleosauroid, Indosinosuchus, Platysuchus, Teleosaurus,

894 Mycterosuchus, Bathysuchus and Sericodon); frontal width is broader than orbital width (shared

895 with Plagiophthalmosuchus, Mystriosaurus, Platysuchus, Teleosaurus, Mycterosuchus,

896 Bathysuchus, Pr. cf. bouchardi, Neosteneosaurus, Mac. buffetauti and Mac. mosae); orbits are

897 longitudinal ellipsoid in shape (shared with Plagiophthalmosuchus, the Chinese teleosauroid,

898 Platysuchus, Macrospondylus, Charitomenosuchus, Seldsienean, Proexochokefalos,

899 Deslongchampsina and Neosteneosaurus); the dorsal margins of the orbits are upturned (shared

900 with I. potamosiamensis, Teleosaurus and Mycterosuchus); angular poorly curved (somewhat

901 similar to Plagiophthalmosuchus and Mystriosaurus); mandibular symphysis is over 50\% of the

902 mandible length (shared with Mycterosuchus, Bathysuchus, Macrospondylus,

903 Charitomenosuchus and Seldsienean); retroarticular width subequal to the glenoid fossa (shared

904 with Lemmysuchus and Mac. buffetauti); P1 and P2 are both on the same transverse plane 
905 (shared with Mycterosuchus, Bathysuchus and Sericodon); the premaxilla lateral margins are

906 sub-rectangular, with the P3 alveoli being clearly lateral to the P2 alveoli (shared with

907 Mycterosuchus, Bathysuchus and Sericodon); at least 22 dentary alveolar pairs; premaxillary and

908 anterior maxillary apicobasal length to basal width ratio of the tooth crown is 3 or greater (shared

909 with Macrospondylus and Charitomenosuchus); shallow tuberculum on the dorsal ribs (shared

910 with Macrospondylus and Charitomenosuchus); the proximal region of the humerus is very

911 strongly posteriorly deflected and hooked (shared with Charitomenosuchus and

912 Neosteneosaurus); femoral condyles are relatively the same size (shared with Macrospondylus,

913 Platysuchus and Lemmysuchus); pits on dorsal osteoderms arranged in alternating rows (similar

914 to Bathysuchus); dorsal osteoderms reduced in size and thickness (shared with Bathysuchus).

915 Remarks - Crocodilus priscus (NHMUK PV R 1086) was the first teleosauroid genus to be

916 scientifically named by von Sömmering in 1814. von Meyer (1830) initially presented Aeolodon

917 gen. nov., and prematurely used this genus for comparison with Rhacheosaurus (1831: 176) but

918 did not provide a formal description until his 1832 volume. Comparing the specimen (NHMUK

919 PV R 1086) to the modern gharial, von Meyer (1832) noted the heterodont teeth (which was his

920 basis for the new genus name) and the "limb bones and phalanges [...] appear like in whales". It

921 is also interesting to note that Geoffroy Saint-Hilaire (1831: 48) did not believe that Aeolodon

922 ("le gavial de Sömmering": "Sömmering's gavial") could be referred to as either Teleosaurus or

923 'Steneosaurus' (mainly due to the fact that it was not found in the deposits near Caen, which

924 Geoffroy Saint-Hilaire believed these two genera were restricted to).

925 Despite coming from different localities, the holotype (NHMUK PV R 1086) and referred

926 specimen (MNHN.F.CNJ 78) share the following combination of features: 
927 1. A longirostrine, weakly ornamented skull;

928 2. Protruding orbits;

929 3. Neural spine and centrum of the mid-cervical vertebrae are approximately equal in height;

930 4. Distal coracoid with rounded edges and deep coracoid foramen;

931 5. An elongated ilial process, more so than other teleosauroids (e.g. Charitomenosuchus

$932 \quad$ NHMUK PV R 3806);

933 6. A sub-triangular ischial blade; and

934 7. Reduced dorsal ornamentation on osteoderms, with large, shallow, well-spaced pits.

935

936

Bathysuchus Foffa et al., 2019

937 Type species-Teleosaurus megahinus Hulke, 1871. Now referred to as Bathysuchus

938 megarhinus (Hulke, 1871), Foffa et al., 2019.

939 Etymology — 'Deep water crocodile'. Bathys, or vathys ( $\beta \alpha \theta v \varsigma)$ is Ancient Greek for deep, and

940 suchus is the Latinized form of the Greek soukhos (бoṽ os), meaning crocodile.

941 Diagnosis - same as the only known species (monotypic genus).

942

Bathysuchus megarhinus (Hulke, 1871) Foffa et al., 2019

943

(Fig. 10)

944 Holotype - NHMUK PV OR 43086, a partial rostrum. 
945 Referred material-DORCM G.05067i-v (premaxillae, isolated tooth and partial osteoderm),

946 LPP unnumbered specimen (a partial rostrum, mandible and skull).

947 Age - Aulacostephanus autissiodorensis Sub-Boreal ammonite Zone and A. eudoxus ammonite 948 Zone, late Kimmeridgian, Late Jurassic.

949 Locality—Kimmeridge, Dorset, UK; Francoulés, Quercy, France.

950 Stratigraphic horizon —Dorset succession, lower Kimmeridge Clay Formation, Ancholme

951 Group; between the Quercynum Horizon and the Contejeani Horizon (Hantzpergue \& Lafaurie, 952 1983).

953 Scoring sources - the holotype (NHMUK PV OR 43086) and the unnumbered LPP specimen 954 were studied first-hand. D. Foffa provided high quality photographs of DORCM G.05067i-v, and 955 B. megarhinus was also discussed at great length with D. Foffa.

956 Autapomorphic characters of B. megarhinus - shallow, minor ornamentation on the parietal

957 (nearly imperceptible); considerably pronounced lateral expansion of the premaxilla with 958 rounded, straightened lateral margins; in the mandible, the fifth dentary alveolar pair is

959 posterolaterally oriented and on the posterior end of the mandibular spatula (rather than posterior 960 to the mandibular spatula).

961 Emended diagnosis - longirostrine snout; rostrum narrows immediately anterior to the orbits 962 (shared with I. potamosiamensis, Teleosaurus, Mycterosuchus, Sericodon, Aeolodon and

963 Seldsienean); shallow, inconspicuous ornamentation of the premaxillae and maxillae (similar to 964 the Chinese teleosauroid, Indosinosuchus, Sericodon and Aeolodon); no ornamentation on the 
965 prefrontal (shared with Plagiophthalmosuchus, I. potamosiamensis, Sericodon and Aeolodon);

966 external nares are ' 8 ' shaped in dorsal view (shared with Mystriosaurus, the Chinese

967 teleosauroid, I. potamosiamensis, Mycterosuchus and Aeolodon) and in anterior view (shared

968 with Mystriosaurus, the Chinese teleosauroid, I. potamosiamensis and Aeolodon); external nares

969 are anterodorsally oriented (shared with Plagiophthalmosuchus, the Chinese teleosauroid,

970 Indosinosuchus, Platysuchus, Mycterosuchus, Aeolodon, Bathysuchus and Sericodon); reduced

971 anteroposterior length of the external nares; more than $67 \%$ of total premaxilla length is posterior

972 to the external nares (shared with Plagiophthalmosuchus, the Chinese teleosauroid, I.

973 potamosiamensis, Mycterosuchus, Sericodon and Aeolodon); premaxillary anterior and posterior

974 medial margin of external nares formed by two bulbous projections (shared with

975 Mycterosuchus); the anterior and anterolateral margins of the premaxillae are strongly

976 anteroventrally deflected and extend ventrally (shared with Mystriosaurus, the Chinese

977 teleosauroid, Mycterosuchus and Platysuchus); inconspicuously ornamented maxillary dorsal

978 surface (shared with the Chinese teleosauroid and Aeolodon), consisting of a shallow irregular

979 pattern of ridges and anastomosing grooves; nasal, prefrontal, lacrimal are also inconspicuously

980 ornamented; absence/extremely reduced frontal ornamentation (shared with Aeolodon); the

981 rostrum narrows markedly immediately anterior to the orbits (shared with I. potamosiamensis,

982 Teleosaurus and Mycterosuchus); frontal width is broader than the orbital width (shared with

983 Plagiophthalmosuchus, Mystriosaurus, Platysuchus, Teleosaurus, Mycterosuchus, Aeolodon, Pr.

984 cf. bouchardi, Neosteneosaurus, Mac. buffetauti and Mac. mosae); palatine anterior margin

985 terminates distal to the 20th maxillary alveoli (shared with Mycterosuchus); basioccipital tubera

986 reduced (shared with Plagiophthalmosuchus, Mycterosuchus and Sericodon); mandibular

987 symphysis over 50\% of mandible length (shared with Mycterosuchus, Aeolodon, 
988 Macrospondylus, Seldsienean and Charitomenosuchus); premaxillae with five alveoli (shared 989 with Platysuchus, Teleosaurus and Sericodon); the P1-P2 do not form a couplet (shared with 990 Platysuchus, Mycterosuchus and Sericodon); the P3-P4 do not form a couple (shared with the 991 Chinese teleosauroid); the P1 and P2 alveoli are lateral to each other at the anterior margin of the 992 premaxilla (shared with Mycterosuchus, Sericodon and possibly Aeolodon); the P3 and P4 are 993 aligned on the lateral plane of the external margin more so than P2 (shared with Sericodon); the $994 \mathrm{P} 1$ and $\mathrm{P} 2$ are on the same transverse plane, and the lateral margin between the $\mathrm{P} 2$ and $\mathrm{P} 3$ is sub995 rectangular (shared with Mycterosuchus, Sericodon and Aeolodon); anterior maxillary 996 interalveolar spacing is sub-equal to longer than adjacent alveoli; lack of apical tooth carinae 997 (shared with Sericodon); the pits on the dorsal osteoderms are circular and regularly organised in 998 alternate rows (similar with Aeolodon); dorsal osteoderms reduced in size and thickness (shared 999 with Aeolodon).

1000 Remarks - Steneosaurus megarhinus was initially named and described by Hulke (1871) and 1001 was recently re-described within a new monotypic genus, Bathysuchus, by Foffa et al. (2019).

1002 Due to similar anatomical features of the cranium, stratigraphic horizons, and comparative 1003 measurements of the humerus and femur with Aeolodon, Foffa et al. (2019) concluded that these 1004 two genera were evidence of the first deep water, more pelagic teleosauroids.

1007 Type species-Sericodon jugleri von Meyer, 1845. 


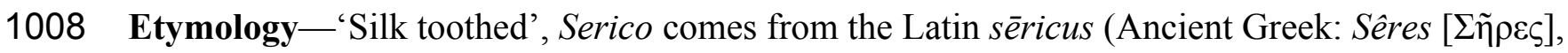

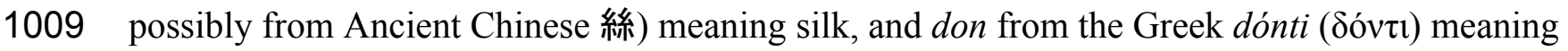
1010 tooth. Refers to the slender, poorly ornamented dentition of this taxon.

1011 Diagnosis - same as the only known species (monotypic genus).

1012

1013 Sericodon jugleri von Meyer, 1845

1014 (Fig. 11)

1015 Type series - Isolated teeth from Hannover (Germany) and Solothurn (Switzerland). Catalogue 1016 numbers currently unknown.

1017 Taxonomic note - von Meyer (1845) initially diagnosed a series of teeth from the 1018 Kimmeridgian of Solothurn and Hannover as the type series of Sericodon; however, it is 1019 unknown if this material is still available, and von Meyer did not designate a holotype. A 1020 lectotype can be proposed for one of the NMS (Switzerland) specimens, but this needs further 1021 clarification. The authors and colleagues plan a thorough description of this specimen, as well as 1022 additional Sericodon material, to allow for a formal designation of a lectotype.

1023 Referred material-BSY006-348, BSY007-134, BSY008-622, SCR010-312, SCR010-1184, 1024 SCR011-2460, SCR011-406, TCH005-151 TCH007-215, VTT006-171 (see Schaefer, Püntener 1025 \& Billon-Bruyat, 2018), as well as LM 16645-46 (anterior mandible), NHMUK PV R 1752, 1026 NZM-PZ R2337, SMF R 431a-b, SMF R 4318 (isolated teeth), unnumbered Göttingen specimen 1027 (partial skull). 
1028 Age - late Kimmeridgian to early Tithonian, Late Jurassic.

1029 Localities - Courtedoux-Bois de Sylleux, Courtedoux-sur Combe Ronde, Courtedoux-Tchâfouè 1030 and Courtedoux-Vâ Tche Tchâ, northwestern Switzerland; Hannover, Germany.

1031 Stratigraphic horizon-Reuchenette Formation.

1032 Scoring sources - Majority of material was scored using Schaefer, Püntener \& Billon-Bruyat 1033 (2018). Additional specimens (LM 16645-46, NHMUK PV R 1752, NRM-PZ R2337, SMF R 1034 431a-b, SMF R 4318, unnumbered Göttingen specimen) were examined first-hand.

1035 Autapomorphic characters of Ser. jugleri - unornamented intertemporal bar; external nares 1036 weakly subcircular in dorsal view; palatal canals extremely shallow; lack of apical enamel 1037 ridges; tuberculum and articular facet of dorsal rib situated close to the lateromedial edge; 1038 posteromedial tuber of femur reduced.

1039 Emended diagnosis - longirostrine snout; rostrum narrows immediately anterior to orbits 1040 (shared with I. potamosiamensis, Teleosaurus, Bathysuchus, Mycterosuchus and Aeolodon); no 1041 conspicuous ornamentation on both the prefrontal (shared with Plagiophthalmosuchus, I.

1042 potamosiamensis, Bathysuchus and Aeolodon) and lacrimal (shared with Plagiophthalmosuchus, 1043 I. potamosiamensis, Aeolodon and Macrospondylus); frontal ornamentation restricted to centre 1044 (shared with Aeolodon, Charitomenosuchus, Seldsienean, Deslongchampsina, Proexochokefalos, 1045 Neosteneosaurus and Machimosaurini); external nares oriented anterodorsally (shared with 1046 Plagiophthalmosuchus, the Chinese teleosauroid, Indosinosuchus, Platysuchus, Mycterosuchus, 1047 Aeolodon and Bathysuchus); over 67\% of total premaxilla length is posterior to the external nares 1048 (shared with Plagiophthalmosuchus, the Chinese teleosauroid, I. potamosiamensis, 
1049 Mycterosuchus, Bathysuchus and Aeolodon); anteromedial projection of the frontal is relatively

1050 broad but becomes immediately mediolaterally thin at the anterior-most part (shared with

1051 Teleosaurus); basioccipital tubera reduced (shared with Plagiophthalmosuchus, Mycterosuchus

1052 and Bathysuchus); five premaxillary alveolar pairs (shared with Platysuchus, Teleosaurus and

1053 Bathysuchus); the P1 and P2 alveoli are lateral to each other at the anterior margin of the

1054 premaxilla (shared with Mycterosuchus, Bathysuchus and possibly Aeolodon); the P3 and P4 are

1055 aligned on the lateral plane of the external margin more so than P2 (shared with Bathysuchus);

1056 the $\mathrm{P} 1$ and $\mathrm{P} 2$ are on the same transverse plane, and the lateral margin between the P2 and P3 is

1057 sub-rectangular (shared with Mycterosuchus, Bathysuchus and Aeolodon); lack of apical carinae

1058 (shared with Bathysuchus); shallow tuberculum (shared with Aeolodon, Macrospondylus and

1059 Charitomenosuchus); postacetabular iliac process elongated (shared with Plagiophthalmosuchus,

1060 Platysuchus, Teleosaurus and Macrospondylus); dorsal osteoderm pits are subcircular and

1061 organised in sub-parallel rows.

1062 Remarks - Sericodon was initially diagnosed by von Meyer (1845) but since the late 1800s has

1063 been considered a subjective junior synonym of 'Steneosaurus' (Sauvage, 1896; Sauvage, 1897-

1064 98; von Huene, 1926; Kuhn, 1936; Steel, 1973; Buffetaut et al., 1985). Sericodon differs from

1065 Bathysuchus in the following characteristics:

1066 1. Sericodon (TCH005-151; Schaefer, Püntener \& Billon-Bruyat, 2018) lacks enamel ridges

1067 on the apices of the dentition, whereas Bathysuchus possesses faint but present enamel

$1068 \quad$ ridges (DORCM G.05067iv);

10692 . The lateral margins of the premaxillae are more expanded and sub-rectangular in

1070 Bathysuchus (NHMUK PV OR 43086; unnumbered LPP specimen). In Sericodon 
1071 (SCR011-406; Schaefer, Püntener \& Billon-Bruyat, 2018) they are less laterally

1072 expanded with more rounded margins;

1073 3. Frontal ornamentation is present in Sericodon (SCR010-312; Schaefer, Püntener \&

1074 Billon-Bruyat, 2018) but is absent in Bathysuchus (unnumbered LPP specimen), in

1075 specimens of approximately equal size;

1076 4. A distinct groove between the two distinct quadrate condyles is present in Sericodon

1077

(SCR010-312; Schaefer, Püntener \& Billon-Bruyat, 2018), whereas in Bathysuchus

1078 (unnumbered LPP specimen) the groove is nearly non-existent (although this may be due

1079 to preservation); and

1080 5. The P3 alveoli is substantially larger than both the P1 and P2 in Sericodon (SCR011-406;

1081 Schaefer, Püntener \& Billon-Bruyat, 2018). In Bathysuchus (DORCM G.05067i), the P3

1082 is relatively the same size as the P2 and slightly larger than the P1.

1083 6. Finally, Sericodon and Bathysuchus are always stable sister taxa in the phylogeny (see

1084 below), regardless of teleosauroid taxa and/or characters added or removed.

1085

1086

Indosinosuchus Martin et al., 2019

1087 Type species_Indosinosuchus potamosiamensis Martin et al., 2019.

1088 Etymology — 'Indochinese crocodile'. Refers to the Indochinese micro-tectonic block where the

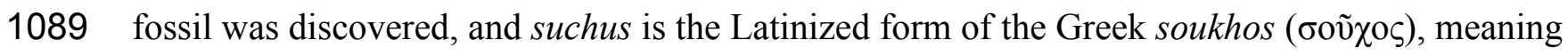
1090 crocodile. 
1091 Diagnosis - tooth row and quadrate condyle are unaligned with quadrate at a lower level, but

1092 both are below the occipital condyle; faint to no conspicuous maxillary ornamentation;

1093 approximately 30 alveoli per dentary.

1094

1095

Indosinosuchus potamosiamensis Martin et al., 2019

1096

(Fig. 12)

1097 Holotype-PRC-11, a complete skull and mandible.

1098 Referred material-PRC-238

1099 Age — Late Jurassic (exact age is unknown, hypothesised to be Tithonian).

1100 Locality_-Pho Noi, Phu Phan range, Kham Muang District, Kalasin Province, northeastern

1101 Thailand.

1102 Stratigraphic horizon - lower part of the Phu Kradung Formation, Khorat Group.

1103 Scoring sources - the holotype (PRC-11) as well as PRC-238 were examined first-hand.

1104 Additional information was gleaned from Martin et al. (2019).

1105 Autapomorphic characters of I. potamosiamensis - extremely anteroposteriorly elongated

1106 posterior nasal processes (reaching the medial margin of the orbit); substantially elongated

1107 anterior process of the nasal, near-parallel to the posterior margin of the antorbital fenestra; the

1108 D2-D3 interalveolar space is longer than that between the D1 and D2. 
1109 Emended diagnosis - mesorostrine snout; tooth row and quadrate condyle unaligned with

1110 quadrate at a lower level, and both below the occipital condyle (shared with I. kalasinensis and

1111 Mycterosuchus); tooth row at a lower level than occipital condyle (shared with

1112 Plagiophthalmosuchus, I. kalasinensis, Platysuchus, Teleosaurus, Mycterosuchus and

1113 Macrospondylus); rostrum narrows immediately anterior to orbits (shared with Teleosaurus,

1114 Mycterosuchus, Aeolodon, Bathysuchus and Sericodon); shallow, irregular maxillary

1115 ornamentation consisting of grooves (similar to the Chinese teleosauroid, Bathysuchus and

1116 Aeolodon); no conspicuous ornamentation on both the prefrontal and lacrimal (similar to

1117 Plagiophthalmosuchus, Aeolodon and Sericodon); frontal ornamentation extends from the centre

1118 to lateral- and anterior-most regions (shared with Plagiophthalmosuchus, the Chinese

1119 teleosauroid, I. kalasinensis, Platysuchus, Teleosaurus, Mycterosuchus, Macrospondylus and

1120 Clovesuurdameredeor); external nares oriented anterodorsally (shared with the Chinese

1121 teleosauroid, I. kalasinensis, Platysuchus, Mycterosuchus, Aeolodon, Bathysuchus and

1122 Sericodon); over $67 \%$ of premaxilla total length is posterior to the external nares (shared with

1123 Plagiophthalmosuchus, the Chinese teleosauroid, Mycterosuchus, Bathysuchus, Sericodon and

1124 Aeolodon); presence of small, oval-shaped antorbital fenestrae; anterior margin of the

1125 supratemporal fossae are noticeably inclined anterolaterally (shared with Mystriosaurus, the

1126 Chinese teleosauroid, I. kalasinensis, Platysuchus, Teleosaurus, Mycterosuchus, Bathysuchus

1127 and Aeolodon); frontal width narrower than orbital width (shared with Charitomenosuchus);

1128 dorsal margins of orbits upturned (shared with Teleosaurus, Mycterosuchus and Aeolodon);

1129 postorbital reaches the orbit posteroventral margin and forms an extensive area of the orbit

1130 ventral margin (shared with Mystriosaurus, the Chinese teleosauroid, Platysuchus, Teleosaurus

1131 and Mycterosuchus); palatine anterior margin terminates level to 17th or 18th maxillary alveoli 
1132 (similar to Charitomenosuchus and Mac. buffetauti); symphysis under half of mandible length,

1133 between 0.45 and 0.5 (shared with Mystriosaurus, Deslongchampsina and Proexochokefalos);

1134 mandibular fenestra anteroposteriorly small and poorly elliptic (similar to Mystriosaurus); at

1135 least 27 maxillary alveolar pairs; third premaxillary alveolus are enlarged relative to adjacent

1136 alveoli (shared with the Chinese teleosauroid); at least 30 dentary alveoli.

1137

1138

Indosinosuchus kalasinensis sp. nov.

1139

(Fig. 13)

1140 Holotype - PRC-239, a nearly complete skull and mandible.

1141 Etymology — the specific epithet refers to the Kalasin Province in northeastern Thailand where

1142 the holotype was found. urn:lsid:zoobank.org:act:2B7DB5BB-1F93-457F-A295-

1143 0409ECCD3998

1144 Age - Late Jurassic (exact age is unknown, hypothesised to be Tithonian).

1145 Locality—Pho Noi, Phu Phan range, Kham Muang District, Kalasin Province, northeastern

1146 Thailand.

1147 Stratigraphic horizon - lower part of the Phu Kradung Formation, Khorat Group.

1148 Scoring Sources-PRC-239 was examined first-hand. 
1149 Autapomorphic characters of $\boldsymbol{I}$. kalasinensis - approximately 64\% of total premaxilla length is

1150 posterior to the external nares; anteroposteriorly thickened postorbital bar.

1151 Emended diagnosis - mesorostrine snout; tooth row and quadrate condyle unaligned with

1152 quadrate at a lower level, and both below the occipital condyle (shared with I. potamosiamensis

1153 and Mycterosuchus); tooth row at a lower level than occipital condyle (shared with

1154 Plagiophthalmosuchus, I. potamosiamensis, Platysuchus, Teleosaurus, Mycterosuchus and

1155 Macrospondylus); premaxilla and maxilla ornamented with shallow ridges (similar to the

1156 Chinese teleosauroid, I. potamosiamensis, Bathysuchus, Sericodon and Aeolodon); frontal

1157 ornamentation extends from the centre to lateral- and anterior-most regions (shared with

1158 Plagiophthalmosuchus, the Chinese teleosauroid, I. potamosiamensis, Platysuchus, Teleosaurus,

1159 Mycterosuchus, Macrospondylus and Clovesuurdameredeor); enlarged premaxillary foramina

1160 lateral to the external nares (similar to Mystriosaurus and Yvridiosuchus); external nares oriented

1161 anterodorsally (shared with Plagiophthalmosuchus, the Chinese teleosauroid, I.

1162 potamosiamensis, Platysuchus, Mycterosuchus, Aeolodon, Bathysuchus and Sericodon);

1163 dorsoventrally deep premaxilla (similar to Mystriosaurus); the anterior and anterolateral

1164 premaxillary margins are orientated anteroventrally and extend ventrally (shared with

1165 Mystriosaurus, the Chinese teleosauroid, I. potamosiamensis, Platysuchus, Teleosaurus,

1166 Mycterosuchus, Bathysuchus and Aeolodon); anterior margin of the supratemporal fossae are

1167 noticeably inclined anterolaterally (shared with Mystriosaurus, the Chinese teleosauroid, I.

1168 potamosiamensis, Platysuchus, Teleosaurus, Mycterosuchus, Bathysuchus and Aeolodon);

1169 frontal width subequal to orbital width (shared with the Chinese teleosauroid, Macrospondylus,

1170 Clovesuurdameredeor, Seldsienean, Yvridiosuchus, Deslongchampsina, Proexochokefalos, Mac. 
1171 hugii and Mac. rex); large, slightly robust teeth (most notably in the posterior dental region) with 1172 a pointed apex (most similar to Mystriosaurus).

1173 Remarks - Martin et al. (2019) initially referred PRC-239 to Indosinosuchus potamosiamensis;

1174 however, we designate PRC-239 as a separate species, I. kalasinensis, as it differentiates from

1175 the holotype (PRC-11) of I. potamosiamensis in several features:

1176 1. Rostrum does not narrow immediately anterior to the orbits in PRC-239, whereas there is 1177 a noticeable narrowing of the rostrum in PRC-11;

$11782 . \quad$ Premaxillary and maxillary neurovascular foramina are nearly 2x larger in PRC-239 than 1179 PRC-11, notably in the premaxillae;

1180 3. External nares 'B'-shaped in anterior view in PRC-239, whereas in PRC-11 they are 1181 somewhat'8-shaped';

1182 4. Premaxillary length posterior to the external nares is between 50-65\% in PRC-239, 1183 whereas in PRC-11 the premaxilla length posterior to the external nares is over $67 \%$;

1184 5. Minimum width of the frontal is subequal to orbital width in PRC-239, whereas in PRC1185 the frontal width is noticeably narrower than the orbital width;

1186 6. Dorsal margin of the orbit flush with the skull dorsal surface in PRC-239 (although this 1187 may be due to dorsoventral crushing) whereas in PRC-11 the dorsal margins of the orbits $1188 \quad$ are prominently upturned; and

1189 7. Poorly elliptic external mandibular fenestra in PRC-239, whereas in I. potamosiamensis 1190 the mandibular fenestra is highly elliptic (anteroposteriorly elongated). 
1192 phylogenetic analyses conducted below, and I. kalasinensis lacks all autapomorphies seen in $I$. 1193 potamosiamensis.

1194

1195

Macrospondylus Jäger, 1831

1196 Type species - Crocodilus bollensis Jäger, 1828. Now referred to as Macrospondylus bollensis 1197 (Jäger, 1828), 1831.

1198 Etymology — 'Large vertebra.' Macro is from the Greek makrýs ( $\mu$ ók $\rho \circ \varsigma$ ) meaning long, and

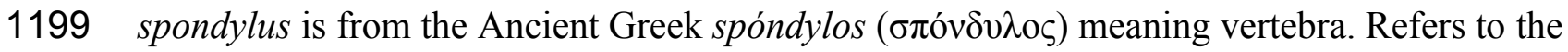
1200 long, amphicoelous vertebrae.

1201 Diagnosis - same as the only known species (monotypic genus).

1202

1203 Macrospondylus bollensis (Jäger, 1828) Jäger, 1831

1204 (Fig. 14)

1205 Holotype - MMG BwJ 595, a partial postcranial skeleton, including dorsal, sacral and anterior 1206 caudal vertebrae, femora, one tibia, one fibula, one pes and disarticulated osteoderms.

1207 Referred material-GPIT-RE-9427; MMG BwJ 565; MMG BwJ 689; NHMUK PV R 324;

1208 NHMUK PV R 756; NHMUK PV R 1088; NHMUK PV R 5703; NHMUK PV OR 14436; 
1209 NHMUK PV OR 14438; NHMW-1848-0031-0001; NHMW-1878-0047-0001; NHMW-1882-

1210 0026-4082; PMU R161; SMNS 18672; SMNS 20280; SMNS 20283; SMNS 51555; SMNS

1211 51563; SMNS 51753; SMNS 51957; SMNS 51984; SMNS 53422; SMNS 58876; SMNS 81699;

1212 SMNS 10000 (all representing partial skulls, complete or near-complete skeletons); unnumbered 1213 OUMNH partial skull.

1214 Age - early Toarcian, Early Jurassic.

1215 Localities_-Baden-Württemberg, Germany; Yorkshire, UK; Sanem, Luxembourg.

1216 Stratigraphic horizons_-Posidonia Shale Formation; Whitby Mudstone Formation; Harpoceras 1217 serpentinum ammonite Zone ('schistes bitumineux').

1218 Scoring sources - the holotype (MMG BwJ 595), as well as a multitude of specimens from

1219 Germany, England and Luxembourg, were studied first-hand. Additional photographs were 1220 provided by B. Kear (PMU), M. Manabe (NMNSJ), U. Menkveld-Gfeller (NMBE), L.

1221 Schöllmann (LWL), A. Sennikov (PIN), W. Simpson (FMNH) and G. Wahlefeld (NMR).

1222 Autapomorphic characters of Ma. bollensis - the proximal region of the humerus is strongly

1223 proximodistally elongated and weakly posteriorly hooked; ulna with a well-developed distal 1224 curvature.

1225 Emended diagnosis - longirostrine skull; tooth row at a lower level than the quadrate (shared 1226 with Plagiophthalmosuchus, Platysuchus, Indosinosuchus, Teleosaurus and Mycterosuchus); no 1227 conspicuous ornamentation on the lacrimal (shared with Plagiophthalmosuchus, I.

1228 potamosiamensis, Bathysuchus, Aeolodon and Charitomenosuchus); frontal ornamentation 
1229 extends from the centre to lateral- and anterior-most regions (shared with Plagiophthalmosuchus,

1230 the Chinese teleosauroid, Indosinosuchus, Platysuchus, Teleosaurus, Mycterosuchus and

1231 Clovesuurdameredeor); external nares oriented dorsally (shared with Plagiophthalmosuchus,

1232 Sericodon, Charitomenosuchus, Proexochokefalos, Deslongchampsina, Neosteneosaurus and

1233 Machimosaurini); presence of shallow, slightly anteroposteriorly elongated antorbital fenestrae;

1234 no anterolateral expansion or inclination of the supratemporal fenestrae (shared with

1235 Plagiophthalmosuchus, Clovesuurdameredeor, Charitomenosuchus, Seldsienean,

1236 Deslongchampsina, Proexochokefalos, Neosteneosaurus and Machimosaurini); frontal width

1237 subequal to orbital width (shared with the Chinese teleosauroid, I. kalasinensis,

1238 Clovesuurdameredeor, Seldsienean, Deslongchampsina, Proexochokefalos, Yvridiosuchus, Mac.

1239 hugii and Mac. rex); orbit is longitudinal ellipsoid in shape (shared with Plagiophthalmosuchus,

1240 the Chinese teleosauroid, Platysuchus, Aeolodon, Charitomenosuchus, Seldsienean,

1241 Proexochokefalos, Deslongchampsina and Neosteneosaurus); basisphenoid exposed along the

1242 palatal surface, bifurcating the pterygoids (shared with Charitomenosuchus, Deslongchampsina,

1243 Proexochokefalos, Neosteneosaurus, Yvridiosuchus and Lemmysuchus); mandibular symphysis

1244 over 50\% of mandible length (shared with Mycterosuchus, Bathysuchus, Aeolodon, Seldsienean

1245 and Charitomenosuchus); anterior maxillary teeth procumbent (shared with I. kalasinensis,

1246 Platysuchus, Teleosaurus, Sericodon, Aeolodon and Charitomenosuchus); tuberculum of dorsal

1247 rib situated on the medial edge (shared with Platysuchus, Aeolodon and Lemmysuchus); shallow

1248 tuberculum on the dorsal ribs (shared with Sericodon, Aeolodon and Charitomenosuchus);

1249 forelimb shorter than hindlimb by approximately $22-23 \%$ (similar to Platysuchus); tibia shorter

1250 than the femur by approximately $25 \%$ (similar to Platysuchus); femoral condyles are relatively

1251 the same size (shared with Platysuchus, Aeolodon and Lemmysuchus). 
1252 Remarks - the holotype of Macrospondylus bollensis (MMG BwJ 595) was one of the first well

1253 preserved vertebrate fossils housed in a scientific institution, dating back to 1755 (von Meyer,

1254 1831: 196). Johann Georg Gmelin, a chemist and pharmacist for the Royal Churfurstliche

1255 Naturaliengalerie Dresden, acquired it at the beginning of the $18^{\text {th }}$ century. Von Meyer initially

1256 presented the holotype in an 1830 public talk (S. Sachs, pers. comm.), and both Dassdorff (1782)

1257 and Walch (1796) briefly noted it to be a crocodile skeleton (von Meyer, 1831); it was then

1258 described by Cuvier $(1812,1824)$ as the iconic "Gavial de Boll” ("Boll gavial”). Jäger (1828)

1259 then named the specimen Crocodilus bollensis, and von Meyer $(1831,1832)$ defined and

1260 described it as a new genus Macrospondylus. The holotype was badly burned in the Zwinger fire

1261 of May 1849 (during the Burgerliche revolution) but survived. Due to this damage, it has been

1262 suggested that it cannot be referable to other Macrospondylus specimens (M. Wilmsen, pers.

1263 comm.). However, MMG BwJ 595 displays a combination of postcranial features unique to

1264 Macrospondylus (e.g. SMNS 18672; SMNS 51563; SMNS 51753; SMNS 51957):

1265 1. Large, anteroposteriorly elongated and dorsoventrally thin cervical ribs (most posteriorly

1266 placed);

1267 2. Shallow tuberculum on dorsal ribs;

1268 3. Ulna with well-developed, pronounced distal curvature that is noticeably larger than the

1269 distal part;

1270 4. Anteroposteriorly short anterior iliac process;

1271 5. Femoral condyles of relatively same size; and

1272 6. Dorsal osteoderms with a pronounced keel and subcircular, numerous, separated pits. 
1275 Type species-Steneosaurus megistorhynchus Eudes-Deslongchamps, 1866a. Now referred to 1276 as Seldsienean megistorhynchus (Eudes-Deslongchamps, 1866a) comb. nov.

1277 urn:1sid:zoobank.org:act:A5177ED2-1416-4C54-A169-05591DA55D80

1278 Etymology_ 'Rare one'. Seldsīene is Old English for 'rare' or 'seldom seen', and '-an' is Old 1279 English for 'one'. Refers to the rarity of this taxon compared to other Bathonian teleosauroids.

1280 Diagnosis - same as the only known species (monotypic genus).

1281

1282 Seldsienean megistorhynchus (Eudes-Deslongchamps, 1866a) comb. nov.

1283

(Fig. 15)

1284 Holotype - A partial skull and complete mandible initially described by Cuvier (1824), re-

1285 described by Eudes-Deslongchamps (1866a; 1867-69), and presumed destroyed in 1944.

1286 Neotype - MMT P28-1 (a partial skull and mandible, as well as isolated vertebrae, fragmented 1287 elements, and three osteoderms and teeth) (see Godefroit, Vignaud \& Lieger, 1995 for additional 1288 information).

1289 Designation of neotype - herein we formally designate MMT P28-1 as the neotype of Se. 1290 megistorhynchus. In order to be in full accordance of Article 75 of the ICZN Code, specifically 1291 Article 75.3, we make the following statements: 
1292 1. This designation is made with the objective of clarifying the taxonomic status of Se.

1293 megistorhynchus.

1294 2. Our assertion of the characters that we regard as distinguishing Se. megistorhynchus from 1295 other teleosauroid taxa is listed in the species diagnosis below.

1296 3. The neotype can be recognized through both the following diagnosis and Figure 15.

1297 4. The holotype is presumed destroyed in 1944 during the bombing of Caen.

1298 5. The holotype, in addition to a partial skull, included a complete mandible; E. Eudes-

1299 Deslongchamps (1867-69: 217) stated that the holotype of Se. megistorhynchus consisted

$1300 \quad$ of a "Museau très-allonge', grêle, étroit et aplati dans toute sa longueur" ("Very

1301 elongated muzzle, slender, narrow and flattened along its entire length"). As such, the

1302 neotype is consistent with what is known of the former name-bearing type.

1303 6. Unfortunately, the locality of the neotype is not known. However, it and the holotype are 1304 from the same age (Bathonian) and country (France), and have been referred to as the $1305 \quad$ same species.

1306 7. Se. megistorhynchus is a slender, longirostrine form, which differs from the genera 1307 Deslongchampsina (mesorostrine) and Yvridiosuchus (durophagous), which are found in 1308 1309 1310 the same stratigraphic horizon and location. In addition, the neotype displays has several distinct features that differ from Deslongchampsina and Yvridiosuchus (e.g. telescopic orbits).

8. The neotype is the property of an internationally recognized scientific institution at the Musée d'art et d'histoire de Toul (MMT), which maintains a research collection with suitable facilities for preserving name-bearing types and is accessible for study.

1314 Referred material- OUMNH J.1414 (near-complete mandible); LPP.T.1 (partial mandible). 
1315 Age - Bathonian, Middle Jurassic.

1316 Localities - unspecified location in France; Enslow Bridge, Oxfordshire, UK.

1317 Stratigraphic horizons - 'Calcaire de Caen'; Cornbrash Formation, Great Oolite Group.

1318 Scoring Sources - the referred specimens (LPP.T.1 and OUMNH J.1415) were studied first-

1319 hand. Additional information was taken from Eudes-Deslongchamps (1866a; 1867-69).

1320 Autapomorphic characters of Se. megistorhynchus - small, circular, noticeably spaced

1321 ornamentation on prefrontal and lacrimal; extremely interdigitated anterior margin of the

1322 palatines; relatively deep, subcircular neurovascular foramina in the posterior region of the

1323 dentary, seen in lateral view; deep coronoid groove; dorsal osteoderms with large, irregularly

1324 shaped and elongated pits with raised areas in between pits, and a small yet well-developed keel

1325 situated in the middle of the osteoderm.

1326 Emended diagnosis - longirostrine skull; rostrum narrows immediately anterior to the orbits

1327 (shared with I. potamosiamensis, Teleosaurus, Mycterosuchus, Aeolodon, Bathysuchus and

1328 Sericodon); frontal ornamentation restricted to centre (shared with Sericodon, Aeolodon,

1329 Charitomenosuchus, Deslongchampsina, Proexochokefalos, Neosteneosaurus and

1330 Machimosaurini); no anterolateral expansion or inclination of the supratemporal fenestrae

1331 (shared with Plagiophthalmosuchus, Clovesuurdameredeor, Macrospondylus,

1332 Charitomenosuchus, Deslongchampsina, Proexochokefalos, Neosteneosaurus and

1333 Machimosaurini); antorbital fenestra present; frontal width subequal to orbital width (shared with

1334 the Chinese teleosauroid, I. kalasinensis, Clovesuurdameredeor, Macrospondylus,

1335 Deslongchampsina, Proexochokefalos, Yvridiosuchus, Mac. hugii and Mac. rex); orbit is 
1336 longitudinal ellipsoid in shape (shared with Plagiophthalmosuchus, the Chinese teleosauroid,

1337 Platysuchus, Aeolodon, Macrospondylus, Charitomenosuchus, Proexochokefalos,

1338 Deslongchampsina and Neosteneosaurus); mandibular symphysis over 50\% of mandible length

1339 (shared with Mycterosuchus, Bathysuchus, Aeolodon, Macrospondylus and Charitomenosuchus);

1340 over 30 dentary alveoli per side (shared with Plagiophthalmosuchus, Platysuchus, Bathysuchus,

1341 Mycterosuchus and Charitomenosuchus).

1342 Remarks - despite fragmentary material, we consider Seldsienean as a distinct taxon because it

1343 is the only longirostrine form present in the Great Oolite Group (UK) during the Bathonian.

1344

1345

Charitomenosuchus gen. nov.

1346 Type species-Steneosaurus leedsi Andrews, 1909. Now referred to as Charitomenosuchus

1347 leedsi (Andrews, 1909), comb. nov. urn:lsid:zoobank.org:act:DE54456D-A305-4A5D-8209-

1348 A987982B200C

1349 Etymology — 'Graceful crocodile'. Charitoménos ( $\chi \alpha \rho \imath \omega \mu \varepsilon \dot{v}$ os) is Greek for 'graceful'

1350 (referring to the slender, elegant skull of this taxon) and suchus is the Latinized form of the

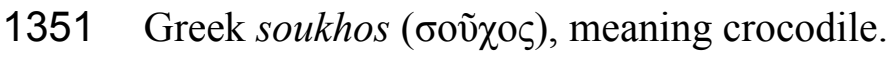

1352 Diagnosis - same as the only known species (monotypic genus).

1353

1354

Charitomenosuchus leedsi (Andrews, 1909) comb. nov. 
1356 Holotype -NHMUK PV R 3320, a nearly complete skull.

1357 Referred material—BRLSI GP1770a-e (a complete skull and mandible); NHMUK PV R 2619

1358 (a complete mandible and additional femora, ilia, ischia, pubes, tibiae, humeri, ulnae, radiae, ribs

1359 [cervical, dorsal], partially preserved vertebrae [two cervical, two dorsal, two sacral] and dorsal

1360 osteoderms); NHMUK PV R 3806 (a nearly complete skeleton); PETMG R179 (complete skull).

1361 Age —Middle Callovian, Middle Jurassic.

1362 Locality—Peterborough, UK.

1363 Stratigraphic horizon-Peterborough Member, Oxford Clay Formation, Ancholme Group.

1364 Scoring Sources - the holotype (NHMUK PV R 3320) as well as all referred specimens

1365 mentioned above were examined first-hand.

1366 Autapomorphic characters of $\boldsymbol{C}$. leedsi-frontal ornamentation consists of circular, spaced

1367 apart pits limited to the centre-most and posterior frontal; strongly interdigitating premaxilla-

1368 maxilla suture; narrow mediolateral supratemporal fenestra width (relative to other

1369 teleosauroids); supratemporal arch dorsal margin subtly concave in lateral view; neural spine

1370 height of anterior thoracic vertebrae is less than centrum height; dorsal osteoderms with large,

1371 subcircular well-spaced pits arranged in a semi-parallel pattern; mediolaterally thickened keel on 1372 sacral osteoderms. 
1373 Emended diagnosis - longirostrine, gracile skull; tooth row and occipital condyle aligned, and

1374 quadrate condyle at a lower level (shared with the Chinese teleosauroid, Proexochokefalos,

1375 Neosteneosaurus and Machimosaurini); skull width less than 26\% of skull length (shared with

1376 Plagiophthalmosuchus, Mycterosuchus, Bathysuchus and Aeolodon); no ornamentation on the

1377 lacrimal (shared with Plagiophthalmosuchus, I. potamosiamensis, Aeolodon and

1378 Macrospondylus); external nares oriented dorsally (shared with Plagiophthalmosuchus,

1379 Macrospondylus, Deslongchampsina, Proexochokefalos, Neosteneosaurus and Machimosaurini);

1380 premaxilla anterior and anterolateral margins are not subvertical (shared with

1381 Plagiophthalmosuchus, Macrospondylus, Andrianavoay, Deslongchampsina, Proexochokefalos,

1382 Neosteneosaurus and Machimosaurini); frontal width narrower than orbital width (shared with $I$.

1383 potamosiamensis); orbit is longitudinal ellipsoid in shape (shared with Plagiophthalmosuchus,

1384 the Chinese teleosauroid, Platysuchus, Aeolodon, Macrospondylus, Seldsienean,

1385 Proexochokefalos, Deslongchampsina and Neosteneosaurus); the anterior process of the jugal is

1386 slender, elongated and extends anteriorly (shared with Clovesuurdameredeor, Proexochokefalos,

1387 Neosteneosaurus and Machimosaurini); palatine anterior margin terminates level to 15 th to 19 th

1388 maxillary alveoli (shared with I. potamosiamensis and Mac. buffetauti); basisphenoid exposed

1389 along the palatal surface, bifurcating the pterygoids (shared with Macrospondylus,

1390 Deslongchampsina, Proexochokefalos, Neosteneosaurus, Yvridiosuchus and Lemmysuchus); the

1391 mandibular symphysis is over $50 \%$ of the mandible length (shared with Bathysuchus,

1392 Mycterosuchus, Macrospondylus, Aeolodon and Seldsienean); mandibular symphysis depth is

1393 very narrow, approximately 4-4.5\% of the mandible length (shared with Mycterosuchus); the P1

1394 is oriented anteriorly whereas the P2 is oriented slightly medially (shared with

1395 Proexochokefalos); over 30 dentary alveoli per side (shared with Plagiophthalmosuchus, 
1396 Platysuchus, Bathysuchus, Mycterosuchus and Seldsienean); slender teeth with weak

1397 mediolateral compression (shared with Macrospondylus); neural spine height of mid-cervical

1398 vertebrae is approximately equal to centrum height (similar to Aeolodon); the tuberculum and

1399 articular facet are situated directly in the dorsal rib (shared with Mycterosuchus); the dorsal rib

1400 tuberculum is shallow (shared with Sericodon, Aeolodon and Macrospondylus); proximal

1401 humerus strongly posteriorly deflected and hooked (similar to Aeolodon, Macrospondylus and

1402 Neosteneosaurus); supraacetabular iliac crest is shallow and poorly pronounced (shared with

1403 Neosteneosaurus, Lemmysuchus and Mac. mosae); postacetabular iliac process is fan-shaped

1404 (shared with Neosteneosaurus, Lemmysuchus and Mac. mosae); tibia approximately 40-50\%

1405 shorter than the femur (shared with Mycterosuchus, Neosteneosaurus, Lemmysuchus and Mac.

1406 mosae); medial femoral condyle larger than lateral femoral condyle (shared with Mycterosuchus,

1407 Neosteneosaurus and Machimosaurus).

1408 Remarks — Both Vignaud (1995) and Mueller-Töwe (2006) considered Mycterosuchus nasutus

1409 to be a synonym of Steneosaurus leedsi (= Charitomenosuchus leedsi).

1410

1411

Deslongchampsina Johnson, Young \& Brusatte, 2019

1412 Type species-Steneosaurus larteti Eudes-Deslongchamps, 1866a. Now referred to as

1413 Deslongchampsina larteti (Eudes-Deslongchamps, 1866a) Johnson, Young \& Brusatte, 2019.

1414 Etymology — Named after Jacques Amand and Eugène Eudes-Deslongchamps, father and son

1415 French naturalists who thoroughly described the holotype specimen and additional teleosauroid 1416 material. 
1417 Diagnosis — same as the only known species (monotypic genus).

1418

1419

Deslongchampsina larteti (Eudes-Deslongchamps, 1866a) Johnson, Young \& Brusatte, 2019

1420

(Fig. 17)

1421 Holotype - A partial skull associated with a partial symphyseal section of the mandible, pelvis,

1422 hindlimb, two vertebrae and dorsal osteoderms. Destroyed in 1944.

1423 Neotype - OUMNH J.29851, a partial skull broken into two pieces. Neotype designation by

1424 Johnson, Young \& Brusatte (2019).

1425 Age-Bathonian, Middle Jurassic.

1426 Localities - Calvados, France; Enslow Bridge, Oxfordshire, UK.

1427 Stratigraphic horizons - 'Fuller's Earth inférieur'; Cornbrash Formation, Great Oolite Group.

1428 Scoring Sources - the neotype (OUMNH J.29851) was studied first-hand.

1429 Autapomorphic characters of $\boldsymbol{D}$. larteti-feeble constriction of the premaxillae posterior to the

1430 external nares, giving the premaxillae a more rounded, 'globular' appearance in dorsal and

1431 ventral views; posterior processes of the nasals are mediolaterally thin; gradual and well-

1432 developed anteroventral sloping of the nasals. See Johnson, Young \& Brusatte (2019) for more

1433 detail. 
1434 Emended diagnosis - mesorostrine snout; frontal ornamentation restricted to the centre (shared

1435 with Sericodon, Aeolodon, Seldsienean, Charitomenosuchus, Proexochokefalos,

1436 Neosteneosaurus and Machimosaurini); external nares oriented dorsally (shared with

1437 Plagiophthalmosuchus, Macrospondylus, Charitomenosuchus, Proexochokefalos,

1438 Neosteneosaurus and Machimosaurini); premaxilla anterior and anterolateral margins are not

1439 sub-vertical (shared with Plagiophthalmosuchus, Macrospondylus, Andrianavoay,

1440 Charitomenosuchus, Proexochokefalos, Neosteneosaurus and Machimosaurini); presence of

1441 large, anteroposteriorly elongated antorbital fenestrae, and internal antorbital fenestra over $25 \%$

1442 of the length of the orbit (shared with Plagiophthalmosuchus); orbit is longitudinal ellipsoid in

1443 shape (shared with Plagiophthalmosuchus, the Chinese teleosauroid, Platysuchus, Aeolodon,

1444 Macrospondylus, Charitomenosuchus, Seldsienean, Proexochokefalos and Neosteneosaurus);

1445 frontal width subequal with orbital width (shared with the Chinese teleosauroid, Mycterosuchus,

1446 Proexochokefalos, Yvridiosuchus, Mac. hugii and Mac. rex); small basioccipital tuberosities

1447 (similar to Bathysuchus); palatine anterior margin terminates distal to the 20th maxillary alveoli

1448 (shared with Charitomenosuchus, Mycterosuchus and Bathysuchus); mandibular symphysis

1449 slightly less than half the mandibular length, between 45 and 50\% (shared with Mystriosaurus, I.

1450 potamosiamensis and Proexochokefalos); deep, well-developed reception pits throughout the

1451 anterior- to mid-maxilla and gradually disappear (similar to Mystriosaurus, Charitomenosuchus

1452 and Proexochokefalos); teeth are robust, slightly curved and weakly-compressed, with pointed

1453 apices and high relief enamel ridges (similar to Neosteneosaurus). 
1456 Type species-Steneosaurus heberti Morel de Glasville, 1876. Now referred to as

1457 Proexochokefalos heberti (Morel de Glasville, 1876), comb. nov.

1458 urn:Isid:zoobank.org:act:FC885641-54CC-421D-84E7-0341140EB704

1459 Etymology — 'Big head with big tuberosities'. Proexochi ( $\pi \rho \circ \varepsilon \xi o \chi \eta ́)$ is Greek for

1460 projection/tuberosity (in an anatomical sense), referring to the large occipital tuberosities that are

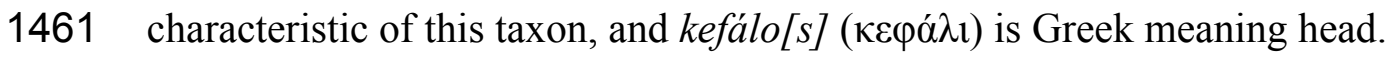

1462 Diagnosis - mesorostrine snout; lack of a midline cavity (= trench) on the nasals; well-developed 1463 occipital tuberosities.

1464

1465

Proexochokefalos heberti (Morel de Glasville, 1876) comb. nov.

1466

(Fig. 18)

1467 Holotype-MNHN.F 1890-13, a complete skull and mandible.

1468 Age - upper Callovian, Middle Jurassic.

1469 Locality_-Villers-sur-mer, Calvados, France.

1470 Stratigraphic horizon-Marnes de Dives Formation.

1471 Scoring sources - the holotype (MNHN.F 1890-13) was studied first-hand.

1472 Autapomorphic characters of Pr. heberti-premaxillae dorsoventrally high in lateral view

1473 (approximately $38 \mathrm{~mm}$ dorsoventral length, from dorsal-most area to tooth row); occipital 
1474 tuberosities large and well-developed; slightly mediolaterally compressed teeth with pointed

1475 apices throughout the dentary series; faint enamel ridges on apical third of teeth; $79-80^{\circ}$ posterior

1476 curvature of the teeth throughout the entire dental series.

1477 Emended diagnosis - mesorostrine skull; tooth row and occipital condyle aligned, and quadrate

1478 condyle at a lower level (shared with the Chinese teleosauroid, Charitomenosuchus, Pr. cf.

1479 bouchardi, Neosteneosaurus and Machimosaurini); frontal ornamentation restricted to centre

1480 (shared with Sericodon, Aeolodon, Charitomenosuchus, Seldsienean, Deslongchampsina,

1481 Neosteneosaurus and Machimosaurini); external nares oriented dorsally (shared with

1482 Plagiophthalmosuchus, Macrospondylus, Charitomenosuchus, Deslongchampsina,

1483 Neosteneosaurus and Machimosaurini); anterior and anterolateral margins of the supratemporal

1484 fenestrae are not sub-vertical (shared with Plagiophthalmosuchus, Macrospondylus,

1485 Andrianavoay, Charitomenosuchus, Deslongchampsina, Neosteneosaurus and Machimosaurini);

1486 flat nasals with no evidence of a midline concavity (shared with Pr. cf. bouchardi); absence of

1487 antorbital fenestrae (shared with Neosteneosaurus and Machimosaurini excluding

1488 Yvridiosuchus); supratemporal fenestra length is twice as long as the anterior width (shared with

$1489 P r$. cf. bouchardi and Neosteneosaurus, and somewhat similar to Machimosaurini); orbit is

1490 longitudinal ellipsoid in shape (shared with Plagiophthalmosuchus, the Chinese teleosauroid,

1491 Platysuchus, Aeolodon, Macrospondylus, Charitomenosuchus, Seldsienean, Pr. cf. bouchardi,

1492 Deslongchampsina and Neosteneosaurus); frontal width sub-equal to orbital width (shared with

1493 the Chinese teleosauroid, I. kalasinensis, Macrospondylus, Clovesuurdameredeor, Seldsienean,

1494 Deslongchampsina, Yvridiosuchus, Mac. hugii and Mac. rex); anterior process of the jugal is

1495 slender and anteriorly elongated (shared with Clovesuurdameredeor, Charitomenosuchus,

1496 Neosteneosaurus and Machimosaurini); mandibular symphysis slightly less than half the 
1497 mandibular length, between 45 and 50\% (shared with Mystriosaurus, I. potamosiamensis and

1498 Deslongchampsina); deep, well-developed reception pits throughout the anterior- to mid-maxilla

1499 and gradually disappear (similar to Mystriosaurus, Charitomenosuchus and Deslongchampsina);

1500 shallow Meckelian groove (shared with Neosteneosaurus and Machimosaurini); sharp dorsal

1501 curvature of the angular (shared with Neosteneosaurus and Machimosaurini); the P1 is oriented

1502 anteriorly whereas the P2 is oriented slightly medially (shared with Proexochokefalos).

1503

1504

Proexochokefalos cf. bouchardi (Sauvage, 1872) comb. nov.

1505

(Fig. 19)

1506 Holotype - A partial specimen initially composed of a skull, mandible and assorted vertebrae

1507 (Vignaud, 1995). Currently missing and/or destroyed.

1508 Referred material-Sauvage (1872); Buffetaut \& Makinsky (1984); Lepage et al. (2008);

1509 SCR010-374 (Schaefer, Püntener \& Billon-Bruyat, 2018).

1510 Age - Kimmeridgian, Late Jurassic.

1511 Localities - Villerville, Calvados, France; Courtedoux-sur Combe Ronde, northwestern

1512 Switzerland.

1513 Stratigraphic horizons - 'Calcaire de Caen'; Reuchenette Formation. 
1514 Scoring sources - Scores were based on specimen photographs from Lepage et al. (2008) and

1515 Schaefer, Püntener \& Billon-Bruyat (2018). Additional information was read from Joleaud

1516 (1928) and Buffetaut \& Makinsky (1984).

1517 Emended diagnosis - mesorostrine skull; tooth row and occipital condyle aligned in the same

1518 plane (similar to the Chinese teleosauroid, Charitomenosuchus, Pr. heberti, Neosteneosaurus and

1519 Machimosaurini); flat nasals with no evidence of a midline concavity (shared with Pr. heberti);

1520 supratemporal fenestrae length is twice as long as width (shared with Pr. heberti and

1521 Neosteneosaurus, and somewhat similar to Machimosaurini); frontal width broader than orbital

1522 width (shared with Plagiophthalmosuchus, Mystriosaurus, Platysuchus, Teleosaurus,

1523 Mycterosuchus, Aeolodon, Bathysuchus, Neosteneosaurus, Mac. buffetauti and Mac. mosae);

1524 orbit is ellipsoid in shape (shared with Plagiophthalmosuchus, the Chinese teleosauroid,

1525 Platysuchus, Aeolodon, Macrospondylus, Charitomenosuchus, Seldsienean, Deslongchampsina,

1526 Pr. heberti and Neosteneosaurus).

1527 Remarks - the mandible of the holotype disappeared, while remnants of the skull material were

1528 initially sent to BHN2 (and was considered the lectotype [presumably BHN2 R 59] by Buffetaut

1529 et al. (1986)). However, this museum was closed in 2003 and the current whereabouts of the

1530 material is unknown. In addition, Vignaud (1995) considered the remaining vertebrae of the

1531 holotype (location also unknown) as the paralectotype, with no formal explanation as to why. In

1532 1892, M. Makinsky discovered the skull figured in Lepage et al. (2008) in the Pictonia baylei

1533 ammonite zone (lower Kimmeridgian) near Villerville (Calvados, France). Buffetaut \&

1534 Makinsky (1984) described it as 'Steneosaurus' cf. bouchardi; currently the location of this

1535 skull, as with all holotype material, is not known (Y. Lepage, pers. comm.). Due to the close 
1536 phylogenetic placement of this taxon to Proexochokefalos heberti, it is currently considered to be 1537 in the same genus.

1538

1539 Steneosaurus Geoffroy Saint-Hilaire, 1825

1540 Type species-Steneosaurus rostromajor Geoffroy Saint-Hilaire, 1825. Type by subsequent 1541 designation (see Johnson, Young \& Brusatte, 2020).

1542 Etymology — 'Narrow lizard.' Steneo is from the Greek sténos ( $\sigma \tau \varepsilon v o ́ \varsigma)$ meaning narrowness 1543 (presumably referring to the elongated maxillae), and saurus is Latin meaning lizard.

1544 Diagnosis - nomen dubium, undiagnostic.

1545

1546 Steneosaurus rostromajor Geoffroy Saint-Hilaire, 1825

1547 (Fig. 20)

1548 Lectotype - MNHN.RJN 134, a partial rostrum. Designated by Johnson, Young \& Brusatte 1549 (2020).

1550 Age - lower Oxfordian, Late Jurassic (Bacheley (1778a, 1778b) and Cuvier $(1808,1812)$ ).

1551 Locality_-Vaches Noires, Calvados, France. 
1552 Stratigraphic horizon-Marnes de Villiers Formation (hypothesized by Bacheley (1778a, 1553 1778b) and Cuvier (1808, 1812)).

1554 Scoring sources - the lectotype (MNHN.RJN 134c-d) was examined first-hand.

1555 Description - maxillae ornamented with numerous, weakly- to strongly developed grooves;

1556 moderately interdigitating premaxilla-maxilla dorsal suture (shared with Mystriosaurus,

1557 Proexochokefalos, Andrianavoay, Neosteneosaurus and Machimosaurini); deep, pronounced

1558 reception pits throughout the entirety of the maxilla (shared with Andrianavoay,

1559 Neosteneosaurus, and Machimosaurini); at least 27 maxillary alveoli; mainly circular, well-

1560 spaced maxillary alveoli throughout the entirety of the rostrum; posterior maxillary alveoli

1561 slightly smaller than anterior maxillary alveoli (similar to Yvridiosuchus); well-developed,

1562 pronounced enamel ridges near the base of the tooth. See Johnson, Young \& Brusatte (2020) for

1563 more detail.

1564 Remarks - initially, the type species of the genus Steneosaurus (MNHN.RJN 134),

1565 Steneosaurus rostromajor Geoffroy Saint-Hilaire, 1825, was composed of a rostrum

1566 (MNHN.RJN 134c-d) and orbital region (MNHN.RJN 134a-b); however, the orbital section

1567 comes from a metriorhynchid. The validity of this taxon has been called into question due to its

1568 fragmentary nature (Eudes-Deslongchamps, 1867-69) and paraphyletic or polyphyletic nature of

1569 Steneosaurus in phylogenetic studies (e.g. Mueller-Töwe, 2006; Ösi et al., 2018; Foffa et al.,

1570 2019; Johnson, Young \& Brusatte, 2019). Currently, only one taxon can hypothetically be

1571 referable to S. rostromajor, Neosteneosaurus; however, due to lack of autapomorphic features,

1572 uncertainty of teleosauroid ontogenetic and sexual dimorphic stages, a generic concept that has 
1573 changed multiple times, and poor preservation, S. rostromajor is currently regarded as a nomen

1574 dubium (Johnson, Young \& Brusatte, 2020).

1575

1576

Andrianavoay gen. nov.

1577 Type species-Steneosaurus baroni Newton, 1893. Now referred to as Andrianavoay baroni

1578 (Newton, 1893), comb. nov. urn:lsid:zoobank.org:act:90C7838E-BE28-4615-BB85-

1579 BB04B67F1304

1580 Etymology — 'Noble crocodile'. Andrian' and voay are Malagasy meaning noble (usually

1581 referring to a prince) and crocodile, respectively.

1582 Diagnosis - same as the only known species (monotypic genus).

1583

1584

Andrianavoay baroni (Newton, 1893) comb. nov.

1585

(Fig. 21)

1586 Holotype - NHMUK PV R 1999, a partial skull and mandible with one associated osteoderm.

1587 Age - Lower Oolite, Bathonian, Middle Jurassic, based on association with Mytilus, Modiola, 1588 Perna and Trochactmonina shells (Newton, 1893).

1589 Locality_-Andranosamonta, northwestern Madagascar. 
1590 Stratigraphic horizon-Unknown.

1591 Scoring sources - the holotype (NHMUK PV R 1999) was examined first-hand.

1592 Autapomorphic characters of $\boldsymbol{A}$. baroni-sparse, small, deep subcircular foramina on the 1593 posterior and lateral margins of the external nares; anteroposteriorly thin posterior-most parietal.

1594 Emended diagnosis - maxilla ornamented with numerous, shallow to deep grooves; premaxilla 1595 anterior and anterolateral margins are not sub-vertical (shared with Plagiophthalmosuchus, 1596 Macrospondylus, Charitomenosuchus, Deslongchampsina, Proexochokefalos, Neosteneosaurus 1597 and Machimosaurini); moderately interdigitating premaxilla-maxilla dorsal suture (shared with 1598 Mystriosaurus, Proexochokefalos, Neosteneosaurus, S. rostromajor and Machimosaurini); 1599 dorsoventrally deep posterior premaxilla (shared with Proexochokefalos); dorsoventrally tall 1600 supraoccipital (shared with Plagiophthalmosuchus, Clovesuurdameredeor and Lemmysuchus);

1601 deep, pronounced reception pits throughout the entirety of the maxilla (shared with S. 1602 rostromajor, Neosteneosaurus and Machimosaurini); osteoderm fragment with large, circular 1603 pits that are well separated from one another.

1604

1605 Neosteneosaurus gen. nov.

1606 Type species_-Steneosaurus edwardsi Eudes-Deslongchamps, 1868a. Now referred to as 1607 Neosteneosaurus edwardsi (Eudes-Deslongchamps, 1868a), comb. nov. 1608 urn:Isid:zoobank.org:act:09ADDEA4-AB2B-40A4-AAFF-19819898532F 
1609 Etymology — 'New Steneosaurus'. 'Neo-' is from the Greek neos (vع́o૬) meaning 'new'. Refers

1610 to the genus this species previously belonged to, Steneosaurus.

1611 Diagnosis — same as the only known species (monotypic genus).

1612

1613

Neosteneosaurus edwardsi (Eudes-Deslongchamps, 1868a) comb. nov.

1614

(Fig. 22)

1615 Holotype -While Eugène Eudes-Deslongchamps (1867-69) described and figured MNHN.RJN

1616118 , he did not formally designate it as the holotype, and included other specimens (syntypes) in

1617 his original description (Brignon, 2018b).

1618 Lectotype-MNHN.RJN 118, a partial skull (see Brignon, 2018b).

1619 Referred material-GPIT-RE-7286 (complete skeleton); NHMUK PV R 2075 (partial skull,

1620 mandible and associated postcrania); NHMUK PV R 2076 (partial mandible and femora, ilia,

1621 tibia, ulna, dorsal and sacral osteoderms); NHMUK PV R 2865 (complete skull, assorted

1622 vertebrae and isolated teeth); NHMUK PV R 3701 (nearly complete skull and mandible, and

1623 partial skeleton); NHMUK PV R 3898 (femur, ilium and ischium); NRM-PZ R.144 (a partial

1624 sacral vertebra); NRM-PZ R.2053 (tibia); NRM-PZ R.2074 (femur); OUMNH J.29815 (partial

1625 skull); PETMG R175 (complete skeleton); PETMG R178 (nearly complete skeleton); SMF R

1626123 (complete skull and nearly complete mandible).

1627 Age — Middle Callovian, Middle Jurassic. 
1628 Locality-Peterborough, UK.

1629 Stratigraphic horizon — Peterborough Member, Oxford Clay Formation, Ancholme Group.

1630 Scoring sources - the holotype (MNHN.RJN 118), as well as all additional referred specimens, 1631 were examined first-hand.

1632 Autapomorphic characters of N. edwardsi-posterior (distal) teeth with sub-pointed apices 1633 (are not blunt and rounded but significantly less pointed than in anterior [mesial] and middle 1634 teeth); tuberculum and articular facet of the dorsal rib positioned on the lateromedial edge.

1635 Emended diagnosis - mesorostrine snout; tooth row and occipital condyle aligned, and quadrate 1636 condyle at a lower level (shared with the Chinese teleosauroid, Charitomenosuchus, 1637 Proexochokefalos and Machimosaurini); frontal ornamentation restricted to centre (shared with 1638 Sericodon, Aeolodon, Charitomenosuchus, Seldsienean, Deslongchampsina, Proexochokefalos 1639 and Machimosaurini); external nares oriented dorsally (shared with Plagiophthalmosuchus, 1640 Macrospondylus, Charitomenosuchus, Deslongchampsina, Proexochokefalos, and 1641 Machimosaurini); premaxilla anterior and anterolateral margins are not sub-vertical (shared with 1642 Plagiophthalmosuchus, Macrospondylus, Andrianavoay, Charitomenosuchus,

1643 Deslongchampsina, Proexochokefalos and Machimosaurini); moderately interdigitating 1644 premaxilla-maxilla suture, appearing subcircular in shape (shared with Mystriosaurus, 1645 Andrianavoay, S. rostromajor, Lemmysuchus and Machimosaurus); absence of antorbital 1646 fenestrae (shared with Proexochokefalos and Machimosaurini excluding Yvridiosuchus); 1647 supratemporal fenestrae length is twice as long as wide (shared with Proexochokefalos, and 1648 somewhat similar to Machimosaurini); the anterior process of the jugal is slender, elongated and 
1649 extends anteriorly (shared with Clovesuurdameredeor, Proexochokefalos and Machimosaurini);

1650 orbit is longitudinal ellipsoid in shape (shared with Plagiophthalmosuchus, the Chinese

1651 teleosauroid, Platysuchus, Aeolodon, Macrospondylus, Charitomenosuchus, Seldsienean,

1652 Proexochokefalos and Deslongchampsina); frontal width broader than orbital width (shared with

1653 Plagiophthalmosuchus, Mystriosaurus, Platysuchus, Teleosaurus, Mycterosuchus, Bathysuchus,

1654 Aeolodon, Pr. cf. bouchardi, Mac. buffetauti and Mac. mosae); squamosal projects further

1655 posteriorly than occipital condyle (shared with the Chinese teleosauroid and Machimosaurini);

1656 shallow Meckelian groove (shared with Proexochokefalos and Machimosaurini); mandibular

1657 symphysis between 30 to $45 \%$ of the mandibular length; (shared with Machimosaurini); deep,

1658 pronounced reception pits throughout the entirety of the maxilla (shared with Andrianavoay,

1659 Neosteneosaurus, and Machimosaurini); maxillary teeth not procumbent (shared with

1660 Proexochokefalos and Machimosaurini); large, robust, weakly-compressed teeth with a pointed

1661 apex and high relief enamel ridges (similar to Deslongchampsina); postacetabular iliac process is

1662 fan-shaped (shared with Charitomenosuchus, Lemmysuchus and Mac. mosae); tibia

1663 approximately 40-50\% shorter than the femur (shared with Mycterosuchus, Charitomenosuchus,

1664 Lemmysuchus and Mac. mosae); medial femoral condyle larger than lateral femoral condyle

1665 (shared with Mycterosuchus, Charitomenosuchus and Machimosaurus); elongated and

1666 pronounced keel across the entirety of the sacral dorsal osteoderms (shared with Lemmysuchus).

1667

1668

TRIBE Machimosaurini (Jouve et al., 2016)

1669

Yvridiosuchus Johnson, Young \& Brusatte, 2019

Peer) reviewing PDF | (2020:03:47227:2:0:NEW 29 Jul 2020) 
1670 Type species-Steneosaurus boutilieri Eudes-Deslongchamps, 1868b. Now referred to as

1671 Yvridiosuchus boutilieri (Eudes-Deslongchamps, 1868b), Johnson, Young \& Brusatte, 2019.

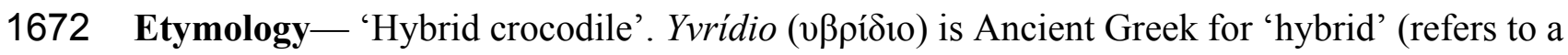

1673 unique combination of non-machimosaurin and machimosaurin teleosauroid symplesiomorphies

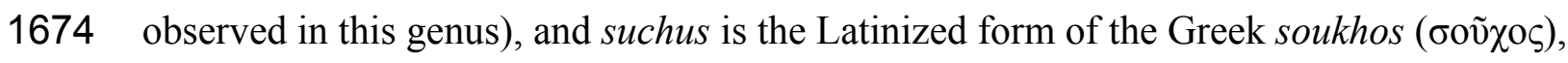

1675 meaning crocodile.

1676 Diagnosis — same as the only known species (monotypic genus).

1677

1678

Yvridiosuchus boutilieri (Eudes-Deslongchamps, 1868b) Johnson, Young \& Brusatte, 2019

1679

(Fig. 23)

1680 Holotype - A skull fragment, figured by Eudes-Deslongchamps (1867-69) and presumed lost or 1681 destroyed (Vignaud, 1995; Johnson, Young \& Brusatte, 2019).

1682 Neotype-OUMNH J.1401, a partial skull. Neotype designation by Johnson, Young \& Brusatte 1683 (2019).

1684 Referred material-OUMNH J.29850 (nearly complete skull and mandible); OUMNH J.1403

1685 (nearly complete skull); OUMNH J.1404 (partial mandible); OUMNH J.1417 (partial mandible)

1686 (see Johnson, Young \& Brusatte, 2019).

1687 Age-Bathonian, Middle Jurassic. 
1688 Localities - Calvados, France; Enslow Bridge, Oxfordshire, UK.

1689 Stratigraphic horizons - 'Sommet de la Grande Oolithe, France; Great Oolite Group, UK.

1690 Scoring sources - the neotype (OUMNH J.1401), as well as all referred specimens mentioned 1691 above, were studied first-hand.

1692 Autapomorphic characters of $\boldsymbol{Y}$. boutilieri - heavily ornamented lacrimal, appearing perforated

1693 in lateral view; extreme elongation of the anterior jugal, so that it participates in the posterior

1694 margin of the antorbital fenestra; orbit subcircular in shape; anterior process of palatine U-

1695 shaped; width of retroarticular process is narrower than the glenoid fossa. See Johnson, Young \& 1696 Brusatte (2019) for more detail.

1697 Emended diagnosis - mesorostrine skull; skull ornamented with numerous conspicuous pits and 1698 grooves (differs from that seen in Mycterosuchus and Mystriosaurus); large and numerous 1699 neurovascular foramina on the premaxillae, maxillae and dentaries (shared with Mystriosaurus 1700 and Machimosaurini); external nares oriented dorsally (shared with Plagiophthalmosuchus, 1701 Macrospondylus, Charitomenosuchus, Proexochokefalos, Deslongchampsina, Neosteneosaurus 1702 and other members of Machimosaurini); premaxilla anterior and anterolateral margins are not 1703 sub-vertical (shared with Plagiophthalmosuchus, Macrospondylus, Andrianavoay, 1704 Charitomenosuchus, Deslongchampsina, Proexochokefalos, Neosteneosaurus and other 1705 members of Machimosaurini); presence of small, deep antorbital fenestrae; frontal width 1706 subequal with orbital width (shared with the Chinese teleosauroid, Mycterosuchus, 1707 Proexochokefalos, Deslongchampsina, Mac. hugii, and Mac. rex); squamosal projects further 1708 posteriorly than occipital condyle (shared with the Chinese teleosauroid, Neosteneosaurus and 
1709 other members of Machimosaurini); shallow Meckelian groove (shared with Proexochokefalos,

1710 Neosteneosaurus and other members of Machimosaurini); sharp dorsoposterior curvature of the

1711 posterior mandibular rami (shared with Proexochokefalos and Lemmysuchus); teeth large and

1712 conical with blunt apices (shared with other members of Machimosaurini); teeth not

1713 mediolaterally compressed (shared with Bathysuchus and other members of Machimosaurini);

1714 carinae heterogeneous with faint denticles (shared with other members of Machimosaurini); teeth

1715 with anastomosing pattern on the apical surface (shared with other members of Machimosaurini);

1716 maxillary teeth not procumbent (shared with Proexochokefalos, Neosteneosaurus and other

1717 members of Machimosaurini).

1718 Remarks - Yvridiosuchus has a long and complicated taxonomic history, including an invalid 1719 species name (Crocodilus oxoniensis; following ICZN Code rules), and OUMNH J.1401 (the

1720 designated neotype) considered by Eudes-Deslongchamps (1867-69) as "appartenant à la même

1721 espèce" ["belonging to the same species"] to the previously destroyed French holotype (Johnson,

1722 Young \& Brusatte, 2019). In addition, Teleosaurus ('Steneosaurus') brevidens Phillips, 1871,

1723 and 'Steneosaurus' meretrix Phizackerely, 1951 (the holotype of T. brevidens), are subjective

1724 junior synonyms of Yvridiosuchus (see Johnson, Young \& Brusatte, 2019 for more information).

1725

1726 Lemmysuchus Johnson et al., 2017

1727 Type species-Steneosaurus obtusidens Andrews, 1909. Now referred to as Lemmysuchus 1728 obtusidens (Andrews, 1909) Johnson et al., 2017. 
1729 Etymology — 'Lemmy’s crocodile'. Lemmy refers to Ian Fraser 'Lemmy' Kilmister, the

1730 deceased founder, lead singer and bassist of the band Motörhead, and suchus is the Latinized

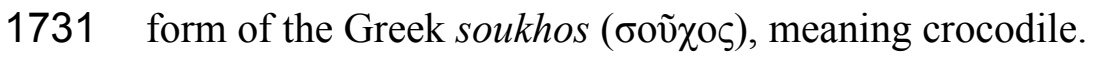

1732 Diagnosis - same as the only known species (monotypic genus).

1733

1734 Lemmysuchus obtusidens (Andrews, 1909) Johnson et al., 2017

1735

(Fig. 24)

1736 Holotype-NHMUK PV R 3168, a nearly complete skeleton including the skull, mandible, 1737 vertebrae, hindlimbs, and multiple osteoderms.

1738 Referred material-LPP.M.21 (a nearly complete skull and mandible); NOTNH FS3361 (a 1739 partial rostrum); PETMG R39 (a rostral-orbital section).

1740 Age-Middle Callovian, Middle Jurassic.

1741 Locality-Peterborough, UK.

1742 Stratigraphic horizon — Peterborough Member, Oxford Clay Formation, Ancholme Group.

1743 Scoring sources - the holotype (NHMUK PV R 3168) and all referred specimens mentioned 1744 above were studied first-hand.

1745 Autapomorphic characters of $\boldsymbol{L}$. obtusidens - the rostrum external surface is strongly convex,

1746 in particular the nasals; partial or complete fusion of the internasal suture; nasal midline cavity 
1747 poorly developed; eight cervical vertebrae; dorsoventrally curved cervical ribs; anterior process

1748 of ilium is anteroposteriorly shortened; acetabulum is shallow and poorly developed; shallow

1749 supraacetabular crest on the ilium; anterior ischial process reduced; dorsal osteoderms with

1750 small-to-large, irregularly shaped pits that radiate from the centre of the keel and are arranged in

1751 a starburst pattern (to a certain extent similar to Mac. mosae). See Johnson et al. (2017) for more

1752 detail.

1753 Emended diagnosis - mesorostrine skull; external nares oriented dorsally (shared with

1754 Plagiophthalmosuchus, Macrospondylus, Deslongchampsina, Proexochokefalos,

1755 Neosteneosaurus and other members of Machimosaurini); two parallel lines of large, circular

1756 neurovascular foramina on the premaxillae and maxillae, and a clustering of foramina on the

1757 lateral surface of the premaxillae (shared with other members of Machimosaurini); premaxilla

1758 anterior and anterolateral margins are not sub-vertical (shared with Plagiophthalmosuchus,

1759 Macrospondylus, Andrianavoay, Charitomenosuchus, Deslongchampsina, Proexochokefalos,

1760 Neosteneosaurus and other members of Machimosaurini); moderately interdigitating premaxilla-

1761 maxilla suture, appearing subcircular in shape (shared with Mystriosaurus, Andrianavoay,

1762 Neosteneosaurus, S. rostromajor, and Machimosaurus); absence of antorbital fenestrae (shared

1763 with Proexochokefalos, Neosteneosaurus and other members of Machimosaurini excluding

1764 Yvridiosuchus); parallelogram-shaped supratemporal fenestrae (shared with other members of

1765 Machimosaurini); the anterior process of the jugal is slender, elongated and extends anteriorly

1766 (shared with Clovesuurdameredeor, Proexochokefalos, Neosteneosaurus and other members of

1767 Machimosaurini); squamosal project posteriorly to occipital condyle (shared with

1768 Plagiophthalmosuchus, the Chinese teleosauroid, Neosteneosaurus and Yvridiosuchus);

1769 supraoccipital dorsoventrally tall (shared with Plagiophthalmosuchus, Clovesuurdameredeor and 
1770 Andrianavoay); shallow Meckelian groove (shared with Proexochokefalos, Neosteneosaurus and

1771 other members of Machimosaurini); retroarticular process subequal to glenoid fossa width

1772 (shared with Aeolodon and Mac. buffetauti); teeth large and conical with blunt apices (shared

1773 with other members of Machimosaurini); teeth not mediolaterally compressed (shared with

1774 Bathysuchus and other members of Machimosaurini); carinae heterogeneous with faint denticles

1775 (shared with other members of Machimosaurini); teeth with anastomosing pattern on the apical

1776 surface (shared with other members of Machimosaurini); axis lacks diapophyses (shared with

1777 Macrospondylus); three sacral vertebrae (shared with Machimosaurus); dorsal ribs with

1778 pronounced tuberculum (shared with Mycterosuchus, Neosteneosaurus and Machimosaurus);

1779 postacetabular iliac process is fan-shaped (shared with Charitomenosuchus, Neosteneosaurus

1780 and Mac. mosae); posteroventral margin of ischial plate sub-squared (shared with Mac. mosae);

1781 tibia approximately 40-50\% shorter than the femur (shared with Mycterosuchus,

1782 Charitomenosuchus, Neosteneosaurus and Mac. mosae); tibial tuberosity angled ventrally

1783 (shared with Mac. mosae); elongate and pronounced keel on sacral osteoderms (shared with

1784 Neosteneosaurus).

1785 Remarks - the exact location of LPP.M.21, which comes from France, is currently unknown.

1786

1787 GENUS Machimosaurus (von Meyer, 1837) emend. von Meyer, 1838

1788 Type species-Machimosaurus hugii von Meyer, 1837 emend. von Meyer, 1838

1789 Referred species-Machimosaurus buffetauti Young et al., 2015b; Machimosaurus mosae

1790 Sauvage \& Liénard, 1879; Machimosaurus rex Fanti et al., 2016. 
1791 Etymology — 'Pugnacious lizard'. Machimo is derived from the Greek machimoi ( $\mu \alpha \dot{\chi} \chi \mu \circ)$,

1792 meaning pugnacious (having a combative nature, presumably referring to the robust dentition),

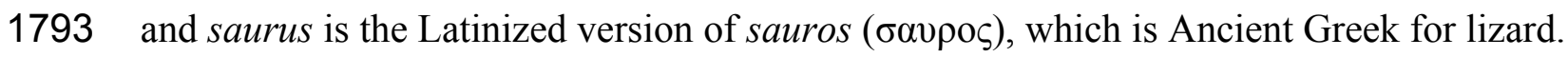

1794 Age - middle Oxfordian to upper Hauterivian/lower Barremian.

1795 Geographical range - Africa (Ethiopia and Tunisia) and Europe (England, France, Germany, 1796 Portugal, Spain and Switzerland).

1797 Generic diagnosis — rostrum wider than high; three alveoli per premaxilla; first premaxillary 1798 alveoli strongly oriented anteroventrally; 18-22 alveoli per maxilla; 19-25 alveoli per dentary; 1799 maximum supratemporal length is greater than $27 \%$ relative to maximum basicranial length; 1800 extreme elongation of the supratemporal fenestrae, with the anteroposterior length twice the 1801 mediolateral length; medial quadrate hemicondyle considerably smaller than the lateral quadrate 1802 hemicondyle; presence of carinae on teeth variable; tall axis neural spine terminating on a plane 1803 dorsal to the pre- and postzygapophyses in lateral view; axis neural spine posteriorly expanded in 1804 lateral view.

1805

1806 Machimosaurus buffetauti Young et al., 2015b

1807 (Fig. 25)

1808 Holotype - SMNS 91415, a complete skull and mandible (as well as in situ teeth) with 1809 associated partial postcranial skeleton including cervical and dorsal vertebrae, one coracoid and 1810 multiple osteoderms. 
1811 Referred material—DFMMh FV 330 (isolated tooth crown); DFMMh FV 541 (isolated tooth

1812 crown); MPV V1600.Bo (anterior region of rostrum and mandible); MPV V1601.Bo (partial

1813 rostrum).

1814 Age - Ataxioceras hypselocyclum Sub-Mediterranean ammonite Zone (=Weißer Jura gamma 2),

1815 Lower Kimmeridgian, Upper Jurassic.

1816 Localities -Am Hörnle Quarry, Neuffen, Baden-Württemberg, Germany; lower Saxony,

1817 Germany; Cricqueboeuf, Normandy, Northern France

1818 Stratigraphic horizons - Lacunosamergel Formation; Langenberg Formation; Calcaires

1819 Coquilliers Formation.

1820 Scoring sources - the holotype (SMNS 91415) was examined first-hand, and additional

1821 information was gleaned from Young et al. (2014, 2015b).

1822 Autapomorphic characters of Mac. buffetauti-anterolateral frontal projections between

1823 nasals and prefrontals; squamosal approximately level with occipital condyle; retroarticular

1824 process is slightly longer than wide; low post-symphyseal tooth count of the dentary; dorsal

1825 margin of the axis neural arch is strongly concave in lateral view; tuberculum and articular facet

1826 of dorsal ribs slightly situated on the medial edge; elongated coracoid glenoid process that

1827 extends considerably from the proximal coracoid, and sub-isosceles triangle-shaped in lateral

1828 view; anterior margin of the coracoid postglenoid process is slightly concave and terminates

1829 approximately in the same frontal plane as the glenoid; posterior margin of the coracoid

1830 postglenoid process is strongly concave and terminates approximately in the same frontal plane 
1831 as the posterior end of the glenoid process; dorsal osteoderms with generally small, irregularly

1832 shaped pits arranged in a random pattern, with a shallow keel.

1833 Emended diagnosis - mesorostrine skull; rostrum wider than high; two parallel lines of large,

1834 circular neurovascular foramina on the premaxillae and maxillae, and a clustering of foramina on

1835 the lateral surface of the premaxillae (shared with Mystriosaurus and members of

1836 Machimosaurini); dentary neurovascular foramina form a relatively straight line (shared with

1837 Mac. mosae); external nares oriented dorsally (shared with Plagiophthalmosuchus,

1838 Macrospondylus, Deslongchampsina, Proexochokefalos, Neosteneosaurus and other members of

1839 Machimosaurini); premaxilla anterior and anterolateral margins are not sub-vertical (shared with

1840 Plagiophthalmosuchus, Macrospondylus, Andrianavoay, Charitomenosuchus,

1841 Deslongchampsina, Proexochokefalos, Neosteneosaurus and other members of

1842 Machimosaurini); premaxilla less than 25\% of rostral length (shared with Mystriosaurus, the

1843 Chinese teleosauroid and Mac. mosae); absence of antorbital fenestrae (shared with

1844 Proexochokefalos, Neosteneosaurus, Lemmysuchus and other members of Machimosaurus);

1845 parallelogram-shaped supratemporal fenestrae (shared with other members of Machimosaurini);

1846 frontal width broader than orbital width (shared with Plagiophthalmosuchus, Mystriosaurus,

1847 Platysuchus, Teleosaurus, Mycterosuchus, Bathysuchus, Aeolodon, Pr. cf. bouchardi,

1848 Neosteneosaurus and Mac. mosae); circular orbits (shared with Mystriosaurus, Indosinosuchus,

1849 Teleosaurus, Mycterosuchus, Clovesuurdameredeor, Lemmysuchus and other members of

1850 Machimosaurus); the anterior process of the jugal is slender, elongated and extends anteriorly

1851 (shared with Clovesuurdameredeor, Proexochokefalos, Neosteneosaurus and Machimosaurini);

1852 quadrates with a single large, circular depression on the dorsal surface close to the hemicondyles;

1853 shallow Meckelian groove (shared with Proexochokefalos, Neosteneosaurus and other members 
1854 of Machimosaurini); retroarticular width is subequal to the glenoid fossa (shared with Aeolodon 1855 and Lemmysuchus); 21-28 maxillary alveolar pairs; deep, pronounced reception pits throughout 1856 the entirety of the maxilla (shared with Andrianavoay, S. rostromajor, Neosteneosaurus and 1857 other members of Machimosaurini); teeth large and conical with blunt apices (shared with other 1858 members of Machimosaurini); teeth not mediolaterally compressed (shared with Bathysuchus

1859 and other members of Machimosaurini); carinae heterogeneous with faint denticles (shared with 1860 other members of Machimosaurini); presence of keeled carinae variable (shared with Mac. hugii 1861 and Mac. rex); teeth with anastomosing pattern on the apical surface (shared with other members 1862 of Machimosaurini).

1863 Remarks - the correct nominal authority is found in the short taxonomic note in Young et al., 1864 2015b, not Young et al. 2014 (where the new taxon was described).

1865

1866 Machimosaurus mosae Sauvage \& Liénard, 1879

1867 (Fig. 26)

1868 Holotype —A skull, destroyed during the First World War. Location and horizon unknown.

1869 Neotype - A partially complete skeleton, labelled as MHNB 1100. Current location unknown.

1870 Referred material-IRSNB (cast of neotype with reconstructed elements added, representing a 1871 complete skeleton); Hua (1999); Young et al (2014). 
1872 Age - Either the Aulacostephanus autissiodorensis Sub-Boreal ammonite Zone, uppermost

1873 Kimmeridgian, or the Gravesia gigas/Pectinaties elegans Sub-Boreal ammonite Zone,

1874 lowermost Tithonian; Late Jurassic (neotype locality).

1875 Neotype locality_Beach near Ambleteuse, Boulonnais, Département du Pas-de-Calais, Nord 1876 Pas-de-Calais, France.

1877 Neotype stratigraphic horizon-Argiles de Châtillon Formation.

1878 Scoring sources-Young et al. (2014). Additional information was gleaned from examining the 1879 large cast of Mac. mosae in the IRSNB exhibit.

1880 Autapomorphic characters of Mac. mosae - anterior palatal margin terminates at 1881 approximately the 11 th to 14 th maxillary alveoli; approximately 17 to 18 alveoli per maxilla; 1882 approximately 19 to 20 alveoli per dentary; coracoid glenoid process very short; anterior edge of 1883 the scapula is strongly concave compared to the posterior edge.

1884 Emended diagnosis - mesorostrine skull; conspicuous grooved-ridged ornamentation of maxilla 1885 (shared with Mac. hugii and Mac. rex); two parallel lines of large, circular neurovascular 1886 foramina on the premaxillae and maxillae, and a clustering of foramina on the lateral surface of 1887 the premaxillae (shared with Mystriosaurus and members of Machimosaurini); dentary 1888 neurovascular foramina form a relatively straight line (shared with Mac. buffetauti); external 1889 nares oriented dorsally (shared with Plagiophthalmosuchus, Macrospondylus, 1890 Deslongchampsina, Proexochokefalos, Neosteneosaurus and other members of 1891 Machimosaurini); premaxilla anterior and anterolateral margins are not subvertical (shared with 1892 Plagiophthalmosuchus, Macrospondylus, Andrianavoay, Charitomenosuchus, 
1893 Deslongchampsina, Proexochokefalos, Neosteneosaurus and other members of

1894 Machimosaurini); premaxilla less than 25\% of rostral length (shared with Mystriosaurus, the

1895 Chinese teleosauroid and Mac. buffetauti); absence of antorbital fenestrae (shared with

1896 Proexochokefalos, Neosteneosaurus, Lemmysuchus and other members of Machimosaurus);

1897 parallelogram-shaped supratemporal fenestrae (shared with other members of Machimosaurini);

1898 frontal width broader than orbital width (shared with Plagiophthalmosuchus, Mystriosaurus,

1899 Platysuchus, Teleosaurus, Mycterosuchus, Bathysuchus, Aeolodon, Pr. cf. bouchardi,

1900 Neosteneosaurus and Mac. buffetauti); circular orbits (shared with Mystriosaurus,

1901 Indosinosuchus, Teleosaurus, Mycterosuchus, Clovesuurdameredeor, Lemmysuchus and other

1902 members of Machimosaurus); shallow Meckelian groove (shared with Proexochokefalos,

1903 Neosteneosaurus and other members of Machimosaurini); deep, pronounced reception pits

1904 throughout the entirety of the maxilla (shared with Andrianavoay, S. rostromajor,

1905 Neosteneosaurus and other members of Machimosaurini); teeth large and conical with blunt

1906 apices (shared with other members of Machimosaurini); teeth not mediolaterally compressed

1907 (shared with Bathysuchus and other members of Machimosaurini); carinae heterogeneous with

1908 faint denticles (shared with other members of Machimosaurini); teeth with anastomosing pattern

1909 on the apical surface (shared with other members of Machimosaurini); three sacral vertebrae

1910 (shared with Lemmysuchus and potentially other members of Machimosaurus); postacetabular

1911 iliac process is fan-shaped (shared with Charitomenosuchus, Neosteneosaurus and

1912 Lemmysuchus); posteroventral margin of ischial plate is sub-square (shared with Lemmysuchus);

1913 tibial tuberosity angled ventrally (shared with Lemmysuchus); dorsal osteoderms ornamented

1914 with small-to-large, irregularly shaped pits that radiate from the centre of the keel and are

1915 arranged in a starburst pattern (similar to an extent in Lemmysuchus). 
1916 Remarks - the diagnosis of Machimosaurus mosae has until recently been uncertain. Sauvage \&

1917 Liénard (1879) initially diagnosed this taxon based on an incomplete skull, mandible and

1918 postcranial material. However, Krebs (1967) viewed it as a junior synonym of Machimosaurus

1919 hugii. Hua (1999) then regarded it as a distinct taxon and proposed a new diagnosis for it, based

1920 on a new specimen from the Kimmeridgian of Boulonnais (northwestern France) containing the

1921 skull, mandible and partial postcranial material. Pierce, Angielczyk \& Rayfield (2009) also

1922 considered Mac. mosae to be distinct from Mac. hugii, due to the position of it within their

1923 geometric morphometric analysis.

1924

However, Martin \& Vincent (2013: 194) criticized Hua’s (1999) and Pierce, Angielczyk

1925 \& Rayfield (2009)'s diagnoses, writing "most of the content of these diagnoses reveal to be

1926 either diagnostic at the genus level or to characterize all Teleosauridae”. Martin \& Vincent

1927 (2013: 195) then showed that high variation in maxillary and dentary tooth counts among the

1928 various Callovian teleosaurids is "sufficient difference to discard such an interpretation (the

1929 synonymy)". Martin \& Vincent (2013) synonymized Mac. mosae with Mac. hugii, thus re-

1930 opening an old debate as to whether Machimosaurus represented a monotypic genus, or if the

1931 differences found between Mac. mosae and Mac. hugii were ontogenetic. However, other

1932 subsequent studies by Vignaud (1995), Hua (1999) and Young at al. (2014) all considered Mac.

1933 mosae to be taxonomically distinct from Mac. hugii. Importantly, Young et al. (2014) outlined

1934 five distinct points that strengthen the separation of Mac. mosae from Mac. hugii:

1935 1. The Mac. mosae neotype is equivalent in size to Mac. buffetauti skulls from France and 1936 Germany;

1937 2. Lack of juvenile characteristics in any of the French and German Mac. buffetauti skulls; 
1938 3. The Mac. mosae neotype exhibits exostoses (the formation of new bone) in the femur, right 1939 pubis, and some caudal vertebrae;

1940 4. There is a 3- to 5-million-year gap between the Mac. mosae neotype and the Mac. hugii 1941 skulls; and

1942 5. Loss of the prearticulars in Mac. mosae, which are present in Mac. hugii.

1943 There are also certain postcranial features that differentiate Mac. mosae and Mac. hugii, 1944 including the shape and size of the coracoid postglenoid and glenoid processes (Young et al., 1945 2014).

1946

1947 Machimosaurus hugii (von Meyer, 1837) emend. von Meyer, 1838

1948 (Fig. 27)

1949 Holotype - von Meyer $(1837,1838)$ never designated a holotype; when establishing Mac. hugii, 1950 he referred to isolated tooth crowns from Solothurn, Switzerland and Kahlenberg, Germany 1951 (syntypes).

1952 Lectotype —NMS 8342, an isolated tooth crown. Designation by Krebs (1967).

1953 Referred material-MCNV-CC-4 (isolated tooth crown); MG-25; MG-8730-1 (two rostral 1954 pieces); MG-8730-2 (occipital section); MG unnumbered; ML 647; ML 491; ML 657; ML 658; 1955 (isolated teeth); Young et al. (2014).

1956 Age - Kimmeridgian, Late Jurassic. 
1957 Localities - Kreuzen Quarry at St. Verena, near Solothurn, Canton Solothurn, Switzerland;

1958 Guimarota coalmine, Leiria, NW Portugal.

1959 Stratigraphic horizon — 'Rätschenbank der Schildkrötenschichten' ("Solothurn Turtle

1960 Limestone, Reuchenette Formation"); Guimarota Strata, Alcobaça Formation.

1961 Scoring sources-MG-8730-1, MG-8730-2 and MG unnumbered were examined first-hand,

1962 along with multiple teeth (e.g. LMH 16386; LMH 16399; MG 25; NZM-PZ R.2358a-g; SMF R

1963 434a-b). Additional information was taken from Young et al. (2014).

1964 Autapomorphic characters of Mac. hugii-external surfaces of the cranial bones are poorly

1965 ornamented, particularly the rostrum and near the orbits; paraoccipital processes greatly

1966 enlarged, mediolaterally elongated and with expanded lateral ends, and are larger than the

1967 exoccipital-opisthotics; in occipital view, the inter-basioccipital tubera notch is a large inverse

1968 'U'-shape; dentary interalveolar spacing uniformly narrow.

1969 Emended diagnosis - mesorostrine skull; groove-ridged ornamentation present along the

1970 maxilla (shared with Mac. mosae and Mac. rex); circular orbits (shared with Mystriosaurus,

1971 Indosinosuchus, Teleosaurus, Mycterosuchus, Clovesuurdameredeor, Lemmysuchus and other

1972 members of Machimosaurus); frontal width sub-equal to orbital width (shared with the Chinese

1973 teleosauroid, I. kalasinensis, Macrospondylus, Clovesuurdameredeor, Seldsienean,

1974 Deslongchampsina, Proexochokefalos, Yvridiosuchus and Mac. rex); parallelogram-shaped

1975 supratemporal fenestrae (shared with other members of Machimosaurini); circular orbits (shared

1976 with Mystriosaurus, Indosinosuchus, Teleosaurus, Mycterosuchus, Clovesuurdameredeor,

1977 Lemmysuchus and other members of Machimosaurus); shallow Meckelian groove (shared with 
1978 Proexochokefalos, Neosteneosaurus and other members of Machimosaurini); deep, pronounced

1979 reception pits throughout the entirety of the maxilla (shared with Andrianavoay, S. rostromajor,

1980 Neosteneosaurus and other members of Machimosaurini); teeth large and conical with blunt

1981 apices (shared with other members of Machimosaurini); teeth not mediolaterally compressed

1982 (shared with Bathysuchus and other members of Machimosaurini); carinae heterogeneous with

1983 faint denticles (shared with other members of Machimosaurini); presence of keeled carinae

1984 variable (shared with Mac. buffetauti and Mac. rex); teeth with anastomosing pattern on the

1985 apical surface (shared with other members of Machimosaurini); pseudodenticles present (shared

1986 with Mac. rex); dorsal osteoderm ornamentation composed of small-to-large, well separated,

1987 irregularly shaped, randomly arranged pits.

1988 Remarks - In response to Young et al. (2014)'s proposal that the genus Machimosaurus

1989 consisted of four distinct species, Martin, Vincent \& Falconnet (2015) wrote a brief rebuttal,

1990 hypothesising that Machimosaurus was monospecific and Mac. hugii was the only representative

1991 of the genus. Foffa et al. (2015) then addressed the rebuttal put forth by Martin, Vincent \&

1992 Falconnet (2015), noting that the authors did not address the monospecifity of Machimosaurus

1993 but rather concentrated on the validity of Mac. buffetauti, suggesting that it is the same as Mac.

1994 mosae and that both should be referred to Mac. hugii (as proposed by Martin \& Vincent [2013]).

1995 Martin, Vincent \& Falconnet (2015) claimed that intraspecific variation or post-mortem

1996 deformation accounted for the diagnoses put forth by Young et al. (2014); however, while

1997 acknowledging that the specimens did undergo some deformation, Foffa et al. (2015) argued that

1998 Young et al. (2014)'s diagnoses consisted of accurate morphological traits. In addition, both

1999 Young et al. (2014) and Foffa et al. (2015) listed six additional factors that differentiated

2000 Machimosaurus species: 
2001 1. Stratigraphy;

2002 2. Basioccipital cross-sections;

2003 3. Comparable size and shape of basioccipital tuberosities;

2004 4. Comparable size and lateral expansion of the paraoccipital processes;

2005 5. Dental morphology, as well as enamel traits; and

2006 6. Tooth counts.

2007

2008

Machimosaurus rex Fanti et al., 2016

2009

(Fig. 28)

2010 Holotype - ONM NG 1-25, 80, 81, and 83-87, comprising a fragmented, partially complete skull

2011 in association with pieces of the atlas-axis complex, two complete dorsal vertebrae, multiple

2012 fragments, and isolated osteoderms and teeth.

2013 Age —late Hauterivian/early Barremian, Early Cretaceous.

2014 Locality_-Touil el Mhahir, Tataouine Governorate, Tunisia.

2015 Stratigraphic horizon—Douiret Sand Member, Douiret Formation.

2016 Scoring sources - the holotype was examined first-hand.

2017 Emended diagnosis - mesorostrine skull; conspicuous groove-ridged ornamentation along the 2018 maxilla (shared with Mac. mosae and Mac. hugii); frontal width sub-equal to orbital width 2019 (shared with the Chinese teleosauroid, I. kalasinensis, Macrospondylus, Clovesuurdameredeor, 
2020 Seldsienean, Deslongchampsina, Proexochokefalos, Yvridiosuchus and Mac. hugii); circular

2021 orbits (shared with Mystriosaurus, Indosinosuchus, Teleosaurus, Mycterosuchus,

2022 Clovesuurdameredeor, Lemmysuchus and other members of Machimosaurus); parallelogram-

2023 shaped supratemporal fenestrae (shared with other members of Machimosaurini); teeth large and

2024 conical with blunt apices (shared with other members of Machimosaurini); teeth not

2025 mediolaterally compressed (shared with Bathysuchus and other members of Machimosaurini);

2026 carinae heterogeneous with faint denticles (shared with other members of Machimosaurini);

2027 presence of keeled carinae variable (shared with Mac. buffetauti and Mac. hugii); teeth with

2028 anastomosing pattern on the apical surface (shared with other members of Machimosaurini);

2029 pseudodenticles present (shared with Mac. hugii); dorsal osteoderm ornamentation consists of

2030 pits with variable size, shape and distribution (similar Lemmysuchus, Mac. buffetauti and Mac.

2031 mosae).

2032 Remarks - While Fanti et al (2016) described this specimen as being Hauterivian in age, the

2033 exact age is unclear, due to uncertainty of the geological age of the area, as well as previously

2034 disregarded biostratigraphic invertebrate fauna (Dridi \& Johnson, 2019; Dridi, 2020). It is also

2035 important to note that Mac. rex does not display any autapomorphic characters, given its

2036 extremely poor preservation.

2037

2038 Character Descriptions

20391.1 New characters pertaining to teleosauroids 
2040 The 38 new characters introduced here were formulated to describe thalattosuchian, specifically

2041 teleosauroid, anatomical variation. These characters are relevant to the interrelationships of

2042 teleosauroids, and many highlight previously unexamined morphological divergence between

2043 two large subclades within the group (see below). These characters are new and are here used in

2044 a cladistic analysis for the first time, and all states (indicated by a number in brackets) are

2045 subsequently figured. Character numbering follows the numbering used in the full list of

2046 characters for the present analysis (see Supplementary Data SD1). More detailed descriptions

2047 and comparisons of all characters have been provided in the Supplementary Data (SD4).

2048 12. Ornamentation on prefrontal in dorsal view: present, with shallow to deep pits and/or grooves

2049 (0), or absent (1) (Fig. 29).

2050

This character was inspired by the variety of ornamentation patterns found on the

2051 prefrontal of teleosauroid taxa. Ornamentation is either absent (state 1) or comes in the form of 2052 shallow to deep pits or shallow to deep, elongated and thin grooves (state 0). State 1 occurs in 2053 very few teleosauroids, including the basal teleosauroid Plagiophthalmosuchus (NHMUK PV 2054 OR 14792), I. potamosiamensis (PRC-11), Aeolodon (MNHN.F.CNJ 78), Sericodon (Schaefer, 2055 Püntener \& Billon-Bruyat, 2018), and Bathysuchus (Foffa et al., 2019). The majority of 2056 teleosauroids are scored as state 0, including the Chinese teleosauroid (IVPP V 10098), 2057 Platysuchus (SMNS 9930), Mycterosuchus (NHMUK PV R 2617), Macrospondylus (GPIT-RE2058 9427; MMG BwJ 565; SMNS 51555), Charitomenosuchus (NHMUK PV R 3320), 2059 Proexochokefalos (MNHN.F 1890-13), and machimosaurins (Yvridiosuchus: OUMNH J.1401; 2060 Lemmysuchus: LPP.M.21; Mac. buffetauti: SMNS 91415). 
2061 13. Ornamentation on lacrimal in dorsal view: present (0), with shallow to deep pits and/or 2062 grooves, or absent (1) (Fig. 29).

2063 As with the above character, the ornamentation displayed on the lacrimal (=lachrymal)

2064 differs between taxa. Ornamentation is either absent (state 1) or comes in the form of shallow to

2065 deep pits, as well as shallow to deep, elongated and thin grooves (state 0 ). The majority of

2066 teleosauroids (Mystriosaurus: NHMUK PV OR 14781; Platysuchus: SMNS 9930;

2067 Mycterosuchus: NHMUK PV R 2617; Proexochokefalos: MNHN.F 1890-13; Lemmysuchus:

2068 NHMUK PV R 3168; Mac. buffetauti: SMNS 91415) exhibit state 0, with some form of

2069 ornamentation being present. State 1 (lack of ornamentation) occurs in six taxa: $I$.

2070 potamosiamensis (PRC-11), Aeolodon (MNHN.F.CNJ 78), Plagiophthalmosuchus (NHMUK PV

2071 OR 14792), Macrospondylus (SMNS 51563), Charitomenosuchus (NHMUK PV R 3320) and

2072 Sericodon (Schaefer, Püntener \& Billon-Bruyat, 2018). As discussed in ch. 12, lack of

2073 ornamentation has previously been attributed to juveniles (e.g. Vignaud, 1995); however, this

2074 character was scored using adult specimens.

2075 15. Frontal, extension of ornamentation: extends from the centre of the frontal to lateral- and 2076 anterior-most regions (0), restricted to centre of the frontal (1) or no ornamentation (2) (Fig. 29).

2077 The frontal of teleosauroids is a single bone that is consistently ornamented throughout

2078 the majority of the group, excluding Bathysuchus (unnumbered LPP specimen) and juveniles

2079 (e.g. SMNS 10 000). Ornamentation either extends from the centre of the frontal to the anterior-

2080 and lateral-most areas (state 0 ) or is restricted to the midline or centre of the frontal (state 1),

2081 with minimal extension. 
2083 OR 49126), Macrospondylus (MMG BwJ 565; SMNS 51563) and many basal teleosauroids (e.g. 2084 Mystriosaurus: NHMUK PV OR 14781; Platysuchus: SMNS 9930), display state 0. The 2085 majority of more derived teleosauroids (e.g. Charitomenosuchus: NHMUK PV R 3320; 2086 Proexochokefalos: MNHN.F 1890-13; Lemmysuchus: LPP.M.21; Mac. buffetauti: SMNS 2087 91415), along with Sericodon (SCR010312 in Schaefer, Püntener \& Billon-Bruyat, 2018) and 2088 Aeolodon (MNHN.F.CNJ 78), share state 1.

2089 It has been suggested that Bathysuchus lacks any frontal ornamentation (Vignaud, 1995), 2090 similar to juvenile individuals. However, there may possibly be weak, nearly unnoticeable pits 2091 and grooves restricted to the midline of the frontal in this taxon (Fig.), in an LPP unnumbered 2092 specimen (Foffa et al., 2019). Due to this uncertainty, this taxon was scored as (?).

2093 43. Premaxilla in dorsal view, the total anteroposterior length relative to total rostrum length is 2094 less than $25 \%(0)$ or approximately $25 \%$ or greater (1) (Fig. 30). total anteroposterior rostrum length of a cranium. When defining the rostral length, this refers to 2097 the length between the anterior-most premaxillae to the anterior orbital margin. In the majority of teleosauroids, the premaxillary anteroposterior length is greater than $209925 \%$ relative to the rostral length (state 1 ). This condition is observed in the basal teleosauroid 2100 Plagiophthalmosuchus (NHMUK PV OR 14792), as well as many longirostrine taxa that are 2101 (e.g. Indosinosuchus: PRC239; Mycterosuchus: NHMUK PV R 2617; Macrospondylus: SMNS 2102 18672; Proexochokefalos: MNHN.F 1890-13; Lemmysuchus: NMHUK PV R 3168). Few 
2103 teleosauroids have a premaxillary anteroposteriorly length that is less than $25 \%$ of the rostral

2104 length (state 0). This is seen in Mac. buffetauti (SMNS 91415) and Mac. mosae (IRSNB cast;

2105 Hua, 1999) as well as Mystriosaurus (NHMUK PV OR 14781) and the Chinese teleosauroid 2106 (IVPP V 10098).

2107 56. Premaxilla in dorsal view, the anterior and posterior medial margins of the external nares are 2108 formed by two bulbous projections, which are either absent (0) or present (1) (Fig. 31).

2109 In most teleosauroids, the medial margins of the external nares are minimally convex 2110 (state 0), causing the external nares to appear D-shaped in dorsal view. This is the condition seen 2111 in the basal Plagiophthalmosuchus (NHMUK PV OR 14792) in addition to Mystriosaurus 2112 (NHMUK PV R OR 14781), Indosinosuchus (PRC11; PRC-239), the Chinese teleosauroid 2113 (IVPP V 10098), Platysuchus (SMNS 9930), Macrospondylus (MMG BwJ 565), 2114 Charitomenosuchus (NHMUK PV R 3806), Proexochokefalos (MNHN.F 1890-13), 2115 Neosteneosaurus (NHMUK PV R 2865) and Machimosaurini (e.g. Lemmysuchus: NHMUK PV 2116 R 3168). In certain taxa, however, both the anterior and posterior margins are strongly convex, and 2118 appear 'bulging' in dorsal view. This condition (state 1) is synapomorphic in a unique clade 2119 containing Mycterosuchus (NHMUK PV R 2617), Bathysuchus (unnumbered LPP specimen) 2120 (Foffa et al., 2019), and possibly Aeolodon (MNHN.F.CNJ 78) (however, specimens of this 2121 taxon are dorsoventrally crushed and slightly distorted, so it is difficult to say with certainty if it 2122 is present). 
2123 58. Premaxilla in dorsal view, the shape of the anteroposterior premaxilla-maxilla contact is

2124 triangular (0), subcircular (1) or 'ragged' (2) (Fig. 31).

2125 In the basal-most form (Plagiophthalmosuchus: NHMUK PV OR 14792), as well as the

2126 Chinese teleosauroid (IVPP V 10098); Indosinosuchus (PRC-11; PRC-239); Platysuchus (SMNS

2127 9930); Aeolodon (MNHN.F.CNJ 78), Mycterosuchus (NHMUK PV R 2617), Bathysuchus

2128 (unnumbered LPP specimen) and Macrospondylus (SMNS 51753; SMNS 51984), the contact is

2129 triangular with slight or no interdigitating areas (state 0 ). An intermediate condition (state 1)

2130 shows the contact to be anteroposteriorly short and subcircular in shape (more posteromedially

2131 horizontally oriented than state 0 ), with a weak to moderate degree of interdigitating regions,

2132 generally close to the midline of the rostrum. This occurs in S. rostromajor (MNHN.RJN 134c-d)

2133 as well as Mystriosaurus (NHMUK PV OR 14781), Andrianavoay (NHMUK PV R 1999),

2134 Proexochokefalos (MNHN.F 1890-13), Neosteneosaurus (NHMUK PV R 2865) and

2135 Machimosaurini (e.g. Lemmysuchus: NHMUK PV R 3168, LPP.M.21). A third condition (state

2136 2) is autapomorphic to Charitomenosuchus (NHMUK PV R 3320, NHMUK PV R 3806): the

2137 premaxilla-maxilla suture is anteroposteriorly elongated, sub-rectangular and highly

2138 interdigitating, giving it a 'ragged'-like appearance.

2139 64. Nasals, elongate posterior process that does not (0) or does (1) contact anterior rim of orbit 2140 (Fig. 32).

2141 In the majority of teleosauroids (e.g. the Chinese teleosauroid: IVPP V 10098;

2142 Platysuchus: SMNS 9930; Mycterosuchus: NHMUK PV R 2617; Lemmysuchus: LPP.M.21),

2143 including the basal-most teleosauroid (Plagiophthalmosuchus: NHMUK PV OR 14792), the

2144 posterior processes of the nasals reach or extend slightly past the anterior rim of the orbits (state 
2145 0). In addition, these processes are positioned medially, slightly mediolaterally thin in the

2146 posterior-most area, and do not come into close contact with the medial orbital margin. However,

2147 I. potamosiamensis (PRC-11) clearly possesses state 1, in which the nasals have extraordinarily

2148 anteroposteriorly elongated posterior processes; these are mediolaterally thin and contacts the

2149 medial rim of the orbit (see Martin et al., 2019).

2150 124. Frontal, anteromedial process shape and length relative to nasals: anterior projection of

2151 frontal is mediolaterally broad and does not extend far anteriorly past anterior orbital rim into

2152 nasals ( 0 ) or anterior projection of frontal is mediolaterally thin and extends anteriorly past

2153 anterior orbital rim into nasals (1) (Fig. 32).

2154 In the majority of teleosauroids, this process is triangular, thin and anteromedially

2155 elongated, usually extending past the anterior orbital margin (state 1). This is seen in taxa such as

2156 the basal-most form Plagiophthalmosuchus (NHMUK PV OR 14792) as well as Mystriosaurus

2157 (NHMUK PV OR 14781), the Chinese teleosauroid (IVPP V 10098), Indosinosuchus taxa (PRC

2158 11; PRC 239), Platysuchus (SMNS 9930), Mycterosuchus (NHMUK PV R 2617), Aeolodon

2159 (MNHN.F.CNJ 78), Macrospondylus (MMG BwJ 565; SMNS 51555), Charitomenosuchus

2160 (NHMUK PV R 3320), Deslongchampsina (OUMNH J.29851), Proexochokefalos (MNHN.F

2161 1890-13), Neosteneosaurus (MNHN.RJN 118; PETMG R178) and Machimosaurini

2162 (Yvridiosuchus OUMNH J.1401; Lemmysuchus LPP.M.21; Mac. buffetauti SMNS 91415). It is

2163 interesting to note that the anteromedial frontal processes in Yvridiosuchus, Indosinosuchus,

2164 Charitomenosuchus and Mac. buffetauti are considerably more elongated and mediolaterally thin

2165 than in the other aforementioned taxa. 
2167 which the anteromedial frontal process is noticeably mediolaterally broadened (giving it a

2168 subcircular appearance in dorsal view) and anteroposteriorly short.

2169 125. Frontal in dorsal view, small anterolateral projections between nasals and prefrontals are

2170 absent (0) or present (1) (Fig. 32).

2171 Most teleosauroids do not have these extra frontal projections; instead, the frontal suture

2172 is flush with that of the posterior nasal processes (state 0 ). This condition is clearly seen in the

2173 basal teleosauroid Plagiophthalmosuchus (NHMUK PV OR 14792) and the Chinese teleosauroid

2174 (IVPP V 10098), Indosinosuchus (PRC-11, PRC-239), Platysuchus (SMNS 9930), Teleosaurus

2175 (MNHN AC 8746), Mycterosuchus (NHMUK PV R 2617), Aeolodon (MNHN.F.CNJ 78),

2176 Macrospondylus (MMG BwJ 565), Clovesuurdameredeor (NHMUK PV OR 49126),

2177 Charitomenosuchus (NHMUK PV R 3320), Deslongchampsina (OUMNH J.29851),

2178 Proexochokefalos (MNHN.F 1890-13), Neosteneosaurus (NHMUK PV R 2865), Yvridiosuchus

2179 (OUMNH J.1401) and Lemmysuchus (LPP.M.21). The presence of these frontal projections is an 2180 apomorphic state, however, in the taxon Mac. buffetauti (Martin \& Vincent, 2013; SMNS

2181 91415), in which they are large, mediolaterally broadened and clearly noticeable (state 1).

2182 167. Jugal anterior process is absent (0) or is slender, elongated and extends anteriorly (1) (Fig. $218333)$. 
2187 the Chinese teleosauroid (IVPP V 10098), Platysuchus (SMNS 9930), Teleosaurus (MNHN AC

2188 8746), Mycterosuchus (NHMUK PV R 2617), Macrospondylus (PMU R161) and

2189 Deslongchampsina (OUMNH J.29851).

2190 In certain teleosauroids, the anterior jugal becomes dorsoventrally curved, narrow and 2191 anteroposteriorly elongated, and extends substantially past the anterior orbital margin, at times 2192 nearly to the posterior region of the antorbital fenestra. This condition (state 1) is present in the 2193 taxa Charitomenosuchus (NHMUK PV R 3320), Neosteneosaurus (MNHN.RJN 118; PETMG 2194 R178), Proexochokefalos (MNHN.F 1890-130) and members of Machimosaurini (e.g. 2195 Yvridiosuchus: OUMNH J.1401).

2196 184. Maxilla in palatal view, shape of anterior maxilla is tapering (subtriangular) (0) or 2197 straightened (sub-rectangular) (1) (Fig. 34).

This character focuses on the anterior premaxilla-maxilla contact in palatal view, which is 2199 positioned parallel to the fourth premaxillary alveolus. State 1 is a synapomorphic character for 2200 members of Teleosauroidea (e.g. the Chinese teleosauroid: IVPP V 10098; Yvridiosuchus:

2201 OUMNH J.1401); the contact is horizontal and straight, and sub-rectangular in shape. This 2202 character is one key difference from Metriorhynchoidea, in which the contact is subtriangular 2203 and anteriorly directed (state 0) (e.g. Metriorhynchus superciliosus: LPP.M.48).

2204 208. Paraoccipital process approximately the same size (0) or substantially larger than the 2205 remainder of the exoccipital-opisthotic (1) (Fig. 35).

2206 Generally, the paraoccipital processes (the posterior-most part of the exoccipital2207 opisthotics) are approximately the same size as the rest of the exoccipital-opisthotic (state 0 ). 
2208 This is seen in the basal form Plagiophthalmosuchus (MNHNL TU515) as well as most

2209 teleosauroids (e.g. the Chinese teleosauroid: IVPP V 10098; Platysuchus: SMNS 9930;

2210 Mycterosuchus: NHMUK PV R 2617; Macrospondylus: SMNS 81699; Charitomenosuchus:

2211 NHMUK PV R 3320; Proexochokefalos: MNHN.F 1890-13; Lemmysuchus: NHMUK PV R

2212 3168). In Mac. hugii (MG-8730-2), the paraoccipital processes are noticeably and substantially

2213 larger than the remaining exoccipital-opisthotics; this condition (state 1) is autapomorphic for

2214 this taxon.

2215 269. Splenials in dorsal view, the excavation of Meckelian groove on the dorsal surface of 2216 symphyseal splenials is deep (0) or shallow (1) (Fig. 36).

2217 This character focuses on the excavation of the Meckelian groove (=canal) seen on the

2218 dorsal surface of the symphyseal splenials. In more basal and longirostrine teleosauroids (e.g.

2219 Mycterosuchus: NHMUK PV R 2617; Macrospondylus: SMNS 53422; Seldsienean: OUMNH

2220 J.1414; Charitomenosuchus: NHMUK PV R 3806), the Meckelian groove is anteroposteriorly

2221 long relative to jaw length and deeply excavated (state 1). In the taxa Proexochokefalos

2222 (MNHN.F 1890-13), Neosteneosaurus (NHMUK PV R 3701) and Machimosaurini (e.g.

2223 Lemmysuchus: LPP.M.21), the Meckelian groove is shallow with little to no excavation (state 0).

2224 270. Angular dorsal curvature is gradual (0) or sharp and abrupt (1) (Fig. 37).

2225 In most teleosauroids, the ventral margin of the angular gradually curves posterodorsally

2226 (state 0). This condition is seen in Indosinosuchus (PRC-11; PRC-239), Platysuchus (SMNS

2227 9930), Sericodon (SCR010-1184 in Schaefer, Püntener \& Billon-Bruyat, 2018), Aeolodon

2228 (MNHN.F.CNJ 78), Macrospondylus (SMNS 51753), Charitomenosuchus (NHMUK PV R 
2229 3806) and Seldsienean (OUMNH J.1414). Both Plagiophthalmosuchus (MNHNL TU515;

2230 NHMUK PV OR 15500) and Mystriosaurus (NHMUK PV OR 14781) also display state 0;

2231 however, the anterior-most angular is straight (horizontally directed), and the dorsoposterior

2232 curvature is poor and limited to the posterior area.

2233 The curvature of the angular differs in Proexochokefalos (MNHN.F 1890-13),

2234 Neosteneosaurus (PETMG R178) and Machimosaurini (Yvridiosuchus: OUMNH J.29850;

2235 Lemmysuchus: NHMUK PV R 3168; Machimosaurus: IRSNB cast, SMNS 91415), in which the

2236 dorsoposterior curvature is immediate, sharp and abrupt (state 1).

2237 291. Maxilla, reception pits are either absent, shallow throughout, or conspicuous only in the

2238 anterior maxilla (0) or pronounced and deep throughout the entirety of the maxilla (1) (Fig. 38).

2239 State 0 includes taxa that have either shallow or absent reception pits on the maxillae;

2240 however, it is important to note that reception pits are present in all teleosauroids, so for the

2241 purposes of this analysis, state 0 of character 291 focuses purely on taxa with shallow reception

2242 pits. These may vary substantially in terms of noticeability; for example, they are present but

2243 near invisible in the basal taxon Plagiophthalmosuchus (MNHNL TU515) and are relatively

2244 small and shallow, disappearing gradually, in most taxa (e.g. Mystriosaurus: NHMUK PV OR

2245 14781; Platysuchus: SMNS 9930; Mycterosuchus: NHMUK PV R 2617;).

2246 In some taxa, the reception pits are deep and noticeable throughout the near-entirety or

2247 entirety of the maxilla, notably so in the anterior and middle regions, although they do become

2248 smaller when progressing posteriorly (state 1). This condition is seen in machimosaurins (e.g.

2249 Lemmysuchus: NHMUK PV R 3618) as well as Andrianavoay (NHMUK PV R 1999), S. 
2250 rostromajor (MNHN.RJN 134c-d, to some extent) and large individuals of Neosteneosaurus 2251 (PETMG R178).

2252 292. Premaxilla, P1-P2 either does not form a couplet and the interalveolar spacing between P12253 P2 and P3-P4 relatively the same size (0) or forms a couplet with the interalveolar spacing 2254 between P1-P2 and P3-P4, with P1-P2 being separated by a thin lamina and P3-P4 being well 2255 separated (1) (Fig. 39).

2257 and fourth (P4), which are positioned posterolaterally. The fifth (P5) premaxillary alveolus 2258 (present in Bathysuchus, Sericodon and Platysuchus) is positioned dorsally in comparison to the 2259 P1 to P4 (Foffa et al., 2019). As such, the interalveolar distance varies between these alveoli. The $2260 \mathrm{P} 1$ and P2 can be well separated in a way similar to that between the P3 and P4; the interalveolar 2261 spacing is large and noticeable, with the adjacent alveoli at a further distance from one another. 2262 This condition (state 0) occurs in Platysuchus (MNHNL TU895), Sericodon (SCR011-406 in 2263 Schaefer, Püntener \& Billon-Bruyat, 2018), Bathysuchus (DORCM G.05067i) and 2264 Mycterosuchus (CAMSM J.1420).

2265 In contrast, in the majority of teleosauroids the P3 and P4 remain separate, but the P1 and 2266 P2 are situated closely together and are either separated by a small, thin interalveolar lamina, or 2267 appear slightly merged together, thereby creating a P1-P2 'couplet' (state 1). This state is seen in 2268 Mystriosaurus (NHMUK PV OR 14781), the Chinese teleosauroid (IVPP V 10098), I. 2269 potamosiamensis (PRC-11) and one subclade of teleosauroids (e.g. Macrospondylus SMNS 2270 18672; Charitomenosuchus: NHMUK PV R 3806; Proexochokefalos: MNHN.F 1890-13; 
2271 Lemmysuchus: NOTNH FS3361). Note that this character is not applicable for taxa that have

2272 fewer than four premaxillary alveoli (Machimosaurus).

2273 293. Premaxilla, P3-P4 couplet is present (0) or absent (1) (Fig. 39).

2274 In most teleosauroids, the interalveolar spacing is generally noticeable and well-

2275 developed between the P3 and the P4, but it is usually small (possibly due to both alveoli being 2276 quite large); the alveoli are therefore closely spaced together, forming a couplet (state 0 ). This is

2277 present in most teleosauroids (e.g. Mystriosaurus: NHMUK PV OR 14781; Platysuchus:

2278 MNHNL TU895; Mycterosuchus: CAMSM J.1420; Macrospondylus SMNS 81699;

2279 Proexochokefalos: MNHN.F 1890-13; Lemmysuchus: NOTNH FS3361). State 1 is found in both 2280 Bathysuchus (NHMUK PV OR 43086, DORCM G.05067i) and the Chinese teleosauroid (IVPP

2281 V 10098), in which the P3-P4 are widely spaced apart from one another, and therefore do not 2282 form a couplet. Note that this character is not applicable for taxa that have fewer than four 2283 premaxillary alveoli (Machimosaurus).

2284 294. Premaxilla in palatal view, both $\mathrm{P} 1$ and $\mathrm{P} 2$ are oriented anteriorly (0), $\mathrm{P} 1$ is oriented 2285 anteriorly and P2 slightly medially (1), or both P1 and P2 are oriented laterally (2) (Fig. 39).

2286 In many teleosauroids, both the P1 and P2 are oriented anteriorly (state 0). This occurs in 2287 Mystriosaurus (NHMUK PV OR 14781), I. potamosiamensis (PRC11), Platysuchus (MNHNL 2288 TU895), Macrospondylus (SMNS 18672), Deslongchampsina (OUMNH J.29851),

2289 Neosteneosaurus (NHMUK PV R 28650), Yvridiosuchus (OUMNH J.1401) and Lemmysuchus 2290 (NOTNH FS3361). In a second condition (state 1), the P1 is oriented anteriorly, but the P2 is 2291 oriented slightly medially. This is seen in Charitomenosuchus (NHMUK PV R 3806) and 
2292 Proexochokefalos (MNHN.F 1890-13). A third condition (state 2), which occurs in Bathysuchus

2293 (Foffa et al., 2019), Sericodon (SCR011-406 in Schaefer, Püntener \& Billon-Bruyat, 2018) and

2294 Mycterosuchus (CAMSM J.1420), is that the P1 and P2 are both strongly oriented laterally,

2295 appearing almost horizontally placed. Note that this character is not applicable for taxa that have

2296 fewer than four premaxillary alveoli (Machimosaurus).

2297 295. Premaxilla, both $\mathrm{P} 1$ and $\mathrm{P} 2$ do not form a couplet and are either not oriented on the anterior

2298 margin of the premaxilla (0) or are oriented on the anterior margin of the premaxilla (1) (Fig.

$229939)$.

2300

In certain teleosauroids, if the P1-P2 alveolar complex does not form a couplet, these two

2301 alveoli are positioned either on or slightly ventral to the anterior margin of the premaxilla. In

2302 Platysuchus (SMNS 9930), the P1 and P2 do not form such a couplet and both alveoli are not

2303 oriented on the anterior margin of the premaxilla (state 0). However, in the genera Bathysuchus

2304 (DORCM G.05067i, unnumbered LPP specimen), Sericodon (SCR011-406 in Schaefer,

2305 Püntener \& Billon-Bruyat, 2018) and Mycterosuchus (CAMSM J.1420), the P1 and P2 do not

2306 form a couplet but are noticeably oriented on the anterior margin of the premaxilla (state 1).

2307 Note that this character is not applicable for taxa that have fewer than four premaxillary alveoli

2308 (Machimosaurus).

2309

296. Premaxilla with no strong lateral expansion (0) or strong lateral expansion so that $\mathrm{P} 3$ and $\mathrm{P} 4$

2310 are aligned on the lateral plane of the external margin, more so than P2 (1) (Fig. 39).

2311 In most teleosauroids, the P3 and P4 are positioned posteriorly to the P1 and P2 and are

2312 aligned on a vertical plane of the lateral margin, whereas the P1 and P2 are aligned more 
2313 laterally, due to little or no lateral expansion of the premaxillae (state 0 ). This condition can be

2314 clearly seen in Plagiophthalmosuchus (NHMUK PV OR 14792), more basal teleosauroids (e.g.

2315 Mystriosaurus: NHMUK PV OR 14781; Platysuchus: MNHNL TU895), and in more derived

2316 teleosauroids (e.g. Charitomenosuchus: NHMUK PV R 3806; Proexochokefalos: MNHN.F

2317 1890-13; Lemmysuchus: LPP.M.21). In select taxa, the premaxillae are laterally expanded, with

2318 the P3 and P4 aligned on a different plane (state 1). This occurs in Bathysuchus (DORCM

2319 G.05067i; unnumbered LPP specimen) and Sericodon (Schaefer, Püntener \& Billon-Bruyat, 2320 2018).

2321 297. Premaxilla, very small first premaxillary alveolus with the second premaxillary alveolus

2322 being much larger (0) or the first and second premaxillary alveoli are relatively the same size (1) 2323 (Fig. 39).

2324 In most teleosauroids, the size of the P1 and P2 are relatively the same, with both being 2325 slightly smaller than the P3 and P4 (which is often the largest, as it houses the large fourth 2326 premaxillary tooth) (state 1). This condition is observed in I. potamosiamensis (PRC-11), 2327 Mycterosuchus (CAMSM J.1420), Bathysuchus (DORCM G.05067i), Deslongchampsina 2328 (OUMNH J.29851), Charitomenosuchus (NHMUK PV R 3806), Proexochokefalos (MNHN.F 2329 1890-13), Neosteneosaurus (NHMUK PV R 2865), Yvridiosuchus (OUMNH J.1401) and 2330 Lemmysuchus (LPP.M.21). In certain teleosauroids, the P1 is considerably smaller than the P2, 2331 with the $\mathrm{P} 1$ being $25 \%$ or less the size of the $\mathrm{P} 2$ (state 0 ). This condition is observed in the 2332 Chinese teleosauroid (IVPP V 10098) and Macrospondylus (SMNS 81699).

2333 339. Dentition, carinae on the apical third of a tooth are present and well pronounced (0) or 2334 absent/weakly pronounced (1) (Fig. 40). 
2335 All known teleosauroids possess carinae (excluding the Chinese teleosauroid IVPP V 2336 10098, Andrianavoay NHMUK PV R 1999, Clovesuurdameredeor NHMUK PV OR 49126 and

2337 P. cf. bouchardi [Lepage et al., 2008], as none have any teeth preserved); in addition, most

2338 teleosauroids have carinae that extend the entire apicobasal length of the tooth, (state 0). These is 2339 seen in the basal form Plagiophthalmosuchus (MNHNL TU515) and Mystriosaurus (NHMUK 2340 PV OR 14781), I. kalasinensis (PRC-239), Mycterosuchus (NHMUK PV R 2617), Aeolodon 2341 (MNHN.F.CNJ 78) Charitomenosuchus (NHMUK PV R 3806), Proexochokefalos (MNHN.F 2342 1890-13) Seldsienean (OUMNH J.1414), Neosteneosaurus (PETMG R178), Lemmysuchus 2343 (NHMUK PV R 3168) and Mac. hugii (MG8730-1). However, two taxa (Bathysuchus: DORCM 2344 G.05067iv; Sericodon: TCH005-151 in Schaefer, Püntener \& Billon-Bruyat, 2018) have carinae 2345 that only extend two-thirds the apicobasal length of the tooth, from the base to the apex and are 2346 absent at the apex (state 1).

2347 340. Dentition, enamel ridges on the apical third of a tooth are absent (0) or present (1) (Fig. 40).

2348 In teleosauroids, the enamel ridges are either faint and/or difficult to see (e.g.

2349 Plagiophthalmosuchus: MNHNL TU515), or noticeable and well-developed (e.g.

2350 Mycterosuchus: NHMUK PV R 2617). Enamel ridges are present on the entirety of the crown, 2351 including the apex (state 1) in the basal-most form Plagiophthalmosuchus (MNHNL TU515), 2352 along with most teleosauroids (e.g. Mystriosaurus: NHMUK PV OR 14781; Mycterosuchus: 2353 NHMUK PV R 2617; Bathysuchus: DORCM G.05067iv; 53422; Charitomenosuchus: NHMUK 2354 PV R 3806; Seldsienean: OUMNH J.1414; Deslongchampsina: OUMNH J.29851;

2355 machimosaurins: NHMUK PV R 3168; NHMW 1846.III.208). Only in one confirmed taxon, 
2356 Sericodon (TCH005-151 in Schaefer, Püntener \& Billon-Bruyat, 2018), are the enamel ridges

2357 absent from the apex (state 0 ).

2358 394. Cervical ribs in lateral view, the anteroposterior ridge of large, more posteriorly placed 2359 cervical ribs is straight (0) or dorsoventrally curved (1) (Fig. 41).

2360 Most teleosauroids that can be scored for this character exhibit T-shaped (in dorsal view) 2361 cervical ribs where the anteroposterior ridge is horizontal or straightened (state 0)(Platysuchus :

2362 SMNS 9930); Mycterosuchus: NHMUK PV R 2617; Charitomenosuchus: NHMUK PV R 3806).

2363 However, in Lemmysuchus (NHMUK PV R 3168), the largest, most posteriorly placed cervical

2364 ribs have a distinct dorsomedial curvature along the anteroposterior ridge, appearing slightly

2365 concave in lateral view (state 1).

2366 395. Dorsal ribs, the positioning of both the tuberculum and articular facet is on the medial edge 2367 (0), directly in the middle (1), or on the lateromedial edge (2) (Fig. 42). In most teleosauroids with preserved dorsal ribs, both the tuberculum and articular facet 2369 are positioned on the medial edge of the rib (state 0). This is observed in Platysuchus (SMNS 9930), Macrospondylus (SMNS 51753, SMNS 18672), Aeolodon (MNHN.F.CNJ 78) and

2371 Lemmysuchus (NHMUK PV R 3168). In two taxa (Mycterosuchus: NHMUK PV R 2617;

2372 Charitomenosuchus: NHMUK PV R 3806), the tuberculum and articular facets have shifted

2373 laterally and are placed directly in the middle of the rib (state 1). In Neosteneosaurus (NHMUK 2374 PV R 3701, PETMG R178), the tuberculum and articular facets have shifted even further 2375 laterally so that they are positioned on the lateromedial edge of the rib (state 2).

2376 396. Dorsal ribs in lateral view, the tuberculum is pronounced (0) or weak (1) (Fig. 42). 
2378 Lemmysuchus (NHMUK PV R 3168) and Mac. buffetauti (SMNS 91415), the tuberculum is

2379 well-developed and pronounced, as large as the capitulum and anteroposteriorly elongated, 2380 giving it an oval shape (state 0). In certain taxa (Sericodon: Schaefer, Püntener \& Billon-Bruyat, 2381 2018; Aeolodon: MNHN.F.CNJ 78; Macrospondylus: SMNS 51753; Charitomenosuchus:

2382 NHMUK PV R 3806), the tuberculum is reduced, small and circular in shape (state 1).

2383 398. Second sacral vertebrae, the anterior margin of the posterior area of the second sacral 2384 vertebra has either a small, non-expanding flange (0) or a large, expanded and projecting flange 2385 (1) (Fig. 43).

In crocodylomorphs, the posterior area of the second sacral vertebra has an anterior

2387 margin that is both anteroposteriorly and dorsoventrally expanded into a projection or 'flange' of 2388 bone, which allows for a secure attachment to the ilium, thus influencing body movement. This 2389 'flange' is either small and non-expanding (state 0), or noticeably expanded and 2390 anteroposteriorly protruding (state 1). All scored teleosauroids exhibit state 1, as there is always 2391 an expanded flange present on the anterior margin; however, the size and development differ. In 2392 the taxa Mycterosuchus (NHMUK PV R 2617), Charitomenosuchus (NHMUK PV R 3806), 2393 Lemmysuchus (NHMUK PV R 3168) and Mac. mosae (Hua, 1999; Young et al., 2014), the 2394 flange is considerably larger, more pronounced and well-developed. In Macrospondylus (MMG 2395 BwJ 595) and Neosteneosaurus (NHMUK PV R 3701) the flange is still present, but it is much 2396 smaller and less obvious.

2397 417. Radius and ulna, the same length (0) or the ulna is longer (1) (Fig. 44). 
2398 In the majority of teleosauroids, the radius and ulna are approximately the same size

2399 (Andrews, 1913), with the ulna being marginally longer (state 0); this is seen in taxa such as

2400 Platysuchus (SMNS 9930), Aeolodon (MNHN.F.CNJ 78), Macrospondylus (SMNS 51563,

2401 SMNS 53422), Charitomenosuchus (NHMUK PV R 3608), Neosteneosaurus (PETMG R178)

2402 and Lemmysuchus (NHMUK PV R 3168). However, in the genus Mycterosuchus (NHMUK PV

$2403 \mathrm{R} 2617$ ) the ulna is roughly $18 \%$ longer than the radius (state 1), which is unusual.

2404 430. Pubis, the shape of distal rim of distal pubic blade is straight and square-like (0) or curved 2405 and rounded (1) (Fig. 45).

2406 In most scored teleosauroids, the ventral (distal) margin of the pubic blade is anteriorly

2407 curved and rounded in lateral view (state 1). This is the case in Charitomenosuchus (NHMUK

2408 PV R 3806), Macrospondylus (SMNS 51957), Neosteneosaurus (PETMG R178), Lemmysuchus

2409 (NHMUK PV R 3168) and Mac. mosae (Hua, 1999; Young et al., 2014). However, in two taxa

2410 the distal rim of the pubic blade is straightened and relatively square-like (state 0 ):

2411 Mycterosuchus (NHMUK PV R 2617) and Platysuchus (SMNS 9930).

2412 431. Pubis, the pubic shaft is shorter (0) or longer (1) than the pubic blade (Fig. 45).

2413 In most teleosauroid taxa, the pubic shaft is either approximately the same length or

2414 slightly anteroposteriorly shorter than the pubic blade (state 0 ). This is the condition seen in six

2415 scored teleosauroids: Macrospondylus (SMNS 51957), Charitomenosuchus (NHMUK PV R

2416 3806), Lemmysuchus (NHMUK PV R 3168), Mac. mosae (Hua, 1999), Platysuchus (SMNS

2417 9930) and Sericodon (SCR010-312 in Schaefer, Püntener \& Billon-Bruyat, 2018). However, the 
2418 pubic shaft is significantly longer (over 50\%) than the pubic blade (state 1) in one taxon

2419 (Mycterosuchus: NHMUK PV R 2617) and represents an apomorphic trait of this genus.

2420 434. Ilium, the anterior iliac process is long and slender (0), or short and robust (1) (Fig. 46).

2421 In most teleosauroids, the anterior iliac process is anteroposteriorly elongated,

2422 mediolaterally slender, and straight with little to no curvature (state 0 ). This is seen in

2423 Platysuchus (SMNS 9930), Teleosaurus (NHMUK PV R 1782a), Sericodon (SCR010-312 in

2424 Schaefer, Püntener \& Billon-Bruyat, 2018), Aeolodon (MNHN.F.CNJ 78), Macrospondylus

2425 (MMG BwJ 565), Charitomenosuchus (NHMUK PV R 3806; Andrews, 1913) and

2426 Neosteneosaurus (PETMG R178). In contrast, state 1 describes the anterior process as

2427 anteroposteriorly shortened, robust and chunky in appearance, with a slight lateral curvature.

2428 This morphology is present in the machimosaurins Lemmysuchus (NHMUK PV R 3168) and

2429 Mac. mosae (Hua, 1999; Young et al., 2014), as well as the basal metriorhynchoid Pelagosaurus

2430 (MNHN.RJN 463) and members of Metriorhynchidae (e.g. Tyrannoneustes lythrodectikos

2431 Young et al., 2013; Cricosaurus lithographicus; Cricosaurus araucanensis [Herrera, Fernández

2432 \& Gasparini, 2013]; Fraas, 1902; Andrews, 1913).

2433 438. Supraacetabular iliac crest is pronounced (0) or shallow and poorly developed (1) in medial 2434 view (Fig. 46).

2435 In non-machimosaurins (e.g. Plagiophthalmosuchus: NHMUK PV OR 14792;

2436 Platysuchus: SMNS 9930; Charitomenosuchus: NHMUK PV R 3806; Neosteneosaurus:

2437 NHMUK PV R 3701, PETMG R178) the supraacetabular crest is enlarged and pronounced,

2438 jutting out laterally and slightly overhanging the acetabulum (state 0 ). In state 1, the 
2439 supraacetabular crest is poorly developed, with either shallow or no outward projection. This is

2440 the case in the machimosaurins Lemmysuchus (NHMUK PV R 3168; Johnson et al., 2017) and

2441 Mac. mosae (Hua, 1999).

2442 449. Ischium, the posteroventral margin of ischial blade is triangular (0) or sub-square (1) (Fig.

2443 47).

2444

In most teleosauroids, the ischial blade is gracile, mediolaterally thin and

2445 anteroposteriorly elongated, with the posteroventral margin having a triangular-like shape (state

2446 0). This morphology is present in Platysuchus (SMNS 9930), Teleosaurus (NHMUK PV R

2447 1638), Mycterosuchus (CAMSM J.1420), Macrospondylus (SMNS 51957), Charitomenosuchus

2448 (NHMUK PV R 3806) and Neosteneosaurus (NHMUK PV R 3701, PETMG R178). A second

2449 condition (state 1) is that the posteroventral margin is noticeably anteroposteriorly shortened and

2450 dorsoventrally broad, giving it a sub-square shape. This state is unique to machimosaurins

2451 (Lemmysuchus: NHMUK PV R 3168; Mac. mosae: ISRNB cast; Hua, 1999; Young et al., 2014).

2452 456. Femur in dorsal view, the anteromedial tuber is present and small (0), or the largest of the 2453 proximal tubera (1) (Fig. 48).

2454

2455 In most teleosauroids, the posteromedial tuber is the largest of the three femoral tubera,

2456 and the anteromedial tuber is present but relatively small (state 0 ). This is the condition seen in

2457 Platysuchus (SMNS 9930), Sericodon (SCR010-312 in Schaefer, Püntener \& Billon-Bruyat,

2458 2018), Aeolodon (MNHN.F.CNJ 78), Macrospondylus (SMNS 18672), Charitomenosuchus

2459 (NHMUK PV R 3806), Neosteneosaurus (PETMG R178) and machimosaurins (Lemmysuchus: 
2460 NHMUK PV R 3168; Machimosaurus: Hua, 1999) The genus Mycterosuchus (NHMUK PV R

2461 2617), however, has an anteromedial tuber that is noticeably well pronounced and well-

2462 developed, and it is the largest of all proximal tubera (state 1).

2463 459. Femur, the distal medial and lateral condyles are the same size (0), or the medial condyle is 2464 larger than the lateral condyle (1) (Fig. 48).

In most teleosauroids, the medial and lateral condyles of the femur are approximately the 2466 same size (state 0). This condition is seen in the basal form Plagiophthalmosuchus (NHMUK PV

2467 OR 14792), as well as Platysuchus (SMNS 9930), Aeolodon (MNHN.F.CNJ 78),

2468 Macrospondylus (SMNS 51555) and Lemmysuchus (NHMUK PV R 3168). In certain

2469 teleosauroid genera, however, the femoral medial condyle is noticeably larger than the femoral

2470 lateral condyle (state 1). This is the case in Mycterosuchus (NHMUK PV R 2617) and

2471 Neosteneosaurus (NHMUK PV R 3701, PETMG R178).

2472 464. Tibia in lateral view, the angle of tibial tuberosity is horizontal (0) or ventral (1) (Fig. 49).

2474 (state 0). This is seen in the basal form Plagiophthalmosuchus (NHMUK PV OR 14792) as well

2475 as Platysuchus (SMNS 9930), Mycterosuchus (NHMUK PV R 2617), Aeolodon (MNHN.F.CNJ

2476 78), Macrospondylus (SMNS 51984), Charitomenosuchus (NHMUK PV R 3806) and

2477 Neosteneosaurus (NHMUK PV R 3701, PETMG R178). In select teleosauroids, the angle of the

2478 tibial tuberosity is strongly ventrally displaced. This condition (state 1) is seen in

2479 machimosaurins (Lemmysuchus: NHMUK PV R 3168; Machimosaurus: IRSNB cast; Hua, 2480 1999). 
2481 466. Calcaneum, the calcaneum tuber is the same size (0) or larger (1) than the astragalus (Fig. $248250)$.

2483 Both the calcaneum and astragalus are approximately the same shapes in all scored

2484 teleosauroids; both tarsal bones are also relatively the same size as one another (state 0 ), with the

2485 calcaneum being marginally larger. This condition is observed in Platysuchus (SMNS 9930),

2486 Macrospondylus (MMG BwJ 565, SMNS 51984), Charitomenosuchus (NHMUK PV R 3806),

2487 Neosteneosaurus (PETMG R178) and Lemmysuchus (NHMUK PV R 3168). However, in

2488 Mycterosuchus (NHMUK PV R 2617) the enlarged calcaneum tuber is noticeably larger than the

2489 astragalus (state 1), by approximately $25 \%$. This condition is currently autapomorphic for this 2490 genus.

2491 489. Sacral dorsal armour (osteoderms), the dorsal keel is elongated and shallow (0) or elongated 2492 and pronounced (1) (Fig. 51).

2493 In certain teleosauroids, the longitudinal ridge (or keel) on the dorsal osteoderms is

2494 anteroposteriorly elongated but shallow (state 0 ). This condition is seen in

2495 Plagiophthalmosuchus (NHMUK PV OR 14792), Platysuchus (SMNS 9930), Teleosaurus

2496 (NHMUK PV R 4207, NHMUK PV OR 32584), Aeolodon (NHMUK PV R 1086,

2497 MNHN.F.CNJ 78), Macrospondylus (SMNS 51563) and Charitomenosuchus (NHMUK PV R

2498 3806). In more derived teleosauroids, the keel of the sacral osteoderms is elongated, well-

2499 developed and thickened (state 1). State 1 is well exemplified in large specimens of

2500 Neosteneosaurus (PETMG R178) as well as the machimosaurin Lemmysuchus (NHMUK PV R $25013168)$. 
2502

25031.2 Previous characters pertaining to teleosauroids

2504 In addition to the 38 new characters described above, several original characters from the 2016

$2505 \mathrm{H}+\mathrm{Y}$ dataset are key in differentiating between various teleosauroid taxa. In particular, 19

2506 characters are anatomically distinct, variant and important in teleosauroids and are described in 2507 detail as follows:

2508 10. Rostrum narrows markedly in dorsal view immediately in front of the orbits (0), or there is 2509 no narrowing (1) (Fig. 52).

2510 In most teleosauroids, the posterior portion of the rostrum will either narrow slightly 2511 mediolaterally or not narrow at all, instead becoming flush with the anterior rim of the orbit 2512 (state 1). This is seen in Plagiophthalmosuchus (NHMUK PV OR 14792), Mystriosaurus 2513 (NHMUK PV OR 14781), the Chinese teleosauroid (IVPP V 10098), Platysuchus (SMNS 9930), 2514 and a particular subclade of teleosauroids (e.g. Macrospondylus MMG BwJ 565;

2515 Charitomenosuchus: NHMUK PV R 3806; Proexochokefalos: MNHN.F 1890-13; Mac.

2516 buffetauti SMNS 91415). In certain teleosauroids, however, there is a distinct and pronounced

2517 narrowing, or mediolateral compression, of the rostrum immediately anterior to the orbits, 2518 causing the dorsal margins of the orbits to become upturned (state 0 ). This condition is in 2519 Mycterosuchus (NHMUK PV R 2617), Aeolodon (MNHN.F.CNJ 78), I. potamosiamensis (PRC2520 11), Teleosaurus (MNHN AC 8746), Sericodon (Schaefer, Püntener \& Billon-Bruyat, 2018), and 2521 Bathysuchus (Foffa et al., 2019). 
2522 27. Neurovascular foramina of the premaxillae/maxillae, represented by a single line of small

2523 sub-circular openings (0), or two lines (one dorsal, one ventral) of large, circular openings (1)

2524 (Fig. 53).

2525

On the lateral premaxillae and maxillae, teleosauroids possess numerous neurovascular

2526 foramina. These openings are possibly involved with multiple mechanoreceptory function such

2527 as prey detection, tactile discrimination or disruption in the surrounding water (e.g. Soares, 2002;

2528 Leitch \& Catania, 2012). In most teleosauroids, the neurovascular foramina are small and

2529 subcircular in shape on both the premaxilla and maxilla, and are generally consistent in size and

2530 number. On the premaxilla, these foramina are restricted to the anteroventral and lateroventral

2531 margins of the external nares. On the ventrolateral surface of the maxilla, dorsal to the tooth row,

2532 they form a single line and are relatively well spaced. This condition (state 0 ) is seen in taxa such

2533 as the basal-most teleosauroid Plagiophthalmosuchus (NHMUK PV OR 14792) and Platysuchus

2534 (SMNS 9930), Mycterosuchus (NHMUK PV R 2617), Macrospondylus (PMU R161), and

2535 Neosteneosaurus (NHMUK PV 2865). Deslongchampsina (OUMNH J. 29851) also has

2536 restricted foramina on the premaxilla as well as a single line on the maxilla; however, the

2537 foramina are larger than those seen in other taxa with state 0 , and are slightly anteroposteriorly

2538 elongated on the maxilla (most notably at the anterior and middle areas of the rostrum).

2539

State 1 is seen in the genus Mystriosaurus (NHMUK PV R 14781) along with members

2540 of Machimosaurini (Yvridiosuchus: OUMNH J.1401, OUMNH J.29850; Lemmysuchus:

2541 NHMUK PV R 3168; Mac. buffetauti: SMNS 91415; Mac. mosae: Young et al., 2014): these

2542 taxa display large, deep, numerous, sub-circular neurovascular foramina (although the foramina

2543 in Mystriosaurus are smaller than in machimosaurins). The premaxillary openings are generally 
2544 circular in shape, located around the ventral, lateral and anteroventral margins of the external

2545 nares and cluster together (especially around the external nares' lateral margins). On the maxilla,

2546 the foramina are more anteroposteriorly elongated and situated in two parallel lines, one dorsal to

2547 the tooth row with an additional line above it (state 1). The foramina are closely spaced together

2548 at the anterior part of the maxilla, but they gradually become more distanced from one another

2549 further posteriorly. In addition, it is interesting to note that the premaxillary foramina are

2550 exceptionally large in Yvridiosuchus (OUMNH J.29850) as well as only around the anteroventral

2551 margin of the external nares in I. kalasinensis (PRC-239).

2552 34. External nares oriented anteriorly or anterodorsally (0), or dorsally (1) (Fig. 54).

2553 In a certain group of predominately Laurasian teleosauroids, the external nares face either

2554 anteriorly or anterodorsally (state 0). This condition occurs in Mystriosaurus (NHMUK PV OR

2555 14781), the Chinese teleosauroid (IVPP V 1009), Mycterosuchus (NHMUK PV R 2617),

2556 Teleosaurus (Eudes-Deslongchamps, 1867-69), Platysuchus (SMNS 9930), Aeolodon

2557 (MNHN.F.CNJ 78), Sericodon (SCR011-406 in Schaefer, Püntener \& Billon-Bruyat, 2018) and

2558 Bathysuchus (unnumbered LPP specimen). In predominately Sub-Boreal/Gondwanan

2559 teleosauroids, the external nares are oriented dorsally (state 1). This is seen in Macrospondylus

2560 (PMU R161), Charitomenosuchus (NHMUK PV R 3806), Deslongchampsina (OUMNH

2561 J.29851), Proexochokefalos (MNHN.F 1890-13), Neosteneosaurus (NHMUK PV R 2865) and

2562 machimosaurins (Yvridiosuchus: OUMNH J.1401; Lemmysuchus: LPP.M.21; Machimosaurus:

2563 SMNS 91415). 
2564 48. Premaxilla in lateral view, the anterior and anterolateral premaxillary margins are not sub-

2565 vertical, or do not extend ventrally (0), or the anterior and anterolateral margins are orientated

2566 anteroventrally and extend ventrally (1) (Fig. 53).

2567 In one teleosauroid subclade, the anterior and anterolateral margins of the premaxilla are

2568 not sub-vertical and do not extend ventrally (state 0 ) when compared to the rest of the

2569 premaxilla; rather, they are anterodorsally curved in a continuous arc throughout. This condition

2570 is seen in the basal teleosauroid Plagiophthalmosuchus (NHMUK PV OR 14792) as well as

2571 Macrospondylus (PMU R161), Charitomenosuchus (NHMUK PV R 3806), Deslongchampsina

2572 (OUMNH J.29851), Proexochokefalos (MNHN.F 1890-13), Andrianavoay (NHMUK PV R

2573 1999), Neosteneosaurus (NHMUK PV R 2865) and Machimosaurini (e.g. Lemmysuchus:

2574 NHMUK PV R 3168). In the second teleosauroid subclade, the anterior and anterolateral

2575 premaxillary margins are strongly oriented anteroventrally and extend ventrally in lateral view,

2576 giving these margins a near-vertical appearance. This condition (state 1) occurs in Mystriosaurus

2577 (NHMUK PV OR 14781), the Chinese teleosauroid (IVPP V 10098), Platysuchus (SMNS 9930),

2578 Mycterosuchus (NHMUK PV R 2617), I. potamosiamensis (PRC-11), Bathysuchus (unnumbered

2579 LPP specimen) and Aeolodon (MNHN.F.CNJ 78). It is particularly well-developed in

2580 Mystriosaurus (NHMUK PV OR 14781) and the Chinese teleosauroid (IVPP V 10098).

2581 83. Antorbital fenestrae/cavity, absent (0) or present (1) (Fig. 52).

2582 In most teleosauroids, a small, slit-like or subcircular antorbital fenestra is present (state

2583 1). This condition is seen in taxa such as Mycterosuchus (NHMUK PV R 2617), Indosinosuchus

2584 (PRC-11, PRC-239), Teleosaurus (MNHN AC 8746), Charitomenosuchus (NHMUK PV R

2585 3806), Macrospondylus (MMG BwJ 565) and Yvridiosuchus (OUMNH J.1401). However, in 
2586 Proexochokefalos (MNHN.F 1890-13), Neosteneosaurus (PETMG R178) and select members of

2587 Machimosaurini (Lemmysuchus: LPP.M.21; Machimosaurus: SMNS 91415; Young et al., 2014)

2588 the antorbital fenestrae (and internal antorbital fossae) are absent (state 0).

2589 86. Antorbital fenestrae/cavity sub-circular (0) or anteroposteriorly elongated (1) in shape (Fig.

$2590 \quad 52)$.

2591 In most teleosauroid taxa, the antorbital fenestra openings are subcircular or sub-oval in 2592 shape (state 0). This condition is seen in Mystriosaurus (NHMUK PV OR 14781), the Chinese 2593 teleosauroid (IVPP V 10098), Indosinosuchus (PRC-11; PRC-239), Platysuchus (SMNS 9930), 2594 Teleosaurus (MNHN AC 8746), Mycterosuchus (NHMUK PV R 2617), Macrospondylus 2595 (SMNS 51555), Charitomenosuchus (NHMUK PV R 3320) and Yvridiosuchus (OUMNH 2596 J.1401). Most notably, in Plagiophthalmosuchus (NHMUK PV OR 14792) and 2597 Deslongchampsina (OUMNH J.29851: Johnson, Young \& Brusatte, 2019), the antorbital 2598 fenestrae are large and anteroposteriorly elongated (state 1), making them appear fully oval- or 2599 teardrop-shaped. Note that this character is not applicable for those taxa that lack antorbital 2600 fenestrae: Proexochokefalos (MNHN.F 1890-13), Neosteneosaurus (PETMG R178), 2601 Lemmysuchus (LPP.M.21) and Machimosaurus (SMNS 91415; Young et al., 2014).

2602 102. Supratemporal fenestrae, shape is either longitudinal ellipsoid or sub-rectangular (0), 2603 square-shaped (regular quadrilateral) (1), transverse (= extended) triangle (2), circular (3), 2604 triangle-shaped (three $60^{\circ}$ points) (4), or parallelogram (5) (Fig. 55). 
2607 than the lateromedial axis (state 0). This is the condition seen in Plagiophthalmosuchus

2608 (NHMUK PV OR 14792; MNHNL TU515), Platysuchus (SMNS 9930), the Chinese

2609 teleosauroid (IVPP V 10098), Mycterosuchus (NHMUK PV R 2617), Aeolodon (MNHN.F.CNJ

2610 78), Sericodon (Schaefer, Püntener \& Billon-Bruyat, 2018), Bathysuchus (unnumbered LPP

2611 specimen), Macrospondylus (MMG BwJ 565), Clovesuurdameredeor (NHMUK PV OR 49126),

2612 Charitomenosuchus (NHMUK PV R 3320), Pr. cf. bouchardi (Lepage et al., 2008),

2613 Proexochokefalos (MNHN.F 1890-13) and Neosteneosaurus (NHMUK PV R 2865, PETMG

2614 R178). Two teleosauroids, I. potamosiamensis (PRC-11) and Teleosaurus (MNHN AC 8746),

2615 show state 1, which is square-shaped supratemporal fenestrae; as with state 0 , the anteroposterior

2616 axis is over 10\% longer than the lateromedial axis. In Machimosaurini (Yvridiosuchus: OUMNH

2617 J.29850; Lemmysuchus: NHMUK PV R 3168; Mac. buffetauti: SMNS 91415; Mac. mosae:

2618 IRSNB cast, Young et al., 2014; Mac. hugii: NMS 7029) the supratemporal fenestrae are

2619 extremely elongated and parallelogram-shaped (state 5), with the lateral and medial margins, and

2620 anterior and posterior margins being sub-parallel. This state is a putative apomorphy within

2621 machimosaurins.

2622 103. Anterior margin shape of supratemporal fenestra, no anterolateral expansion of the

2623 supratemporal fenestrae/fossae (0), or the anterior margin noticeably inclined anterolaterally (1)

2624 (Fig. 55).

2625 In most teleosauroids, the anterior margin of the supratemporal fenestra is not

2626 anterolaterally expanded, and the anterolateral corners of the supratemporal fossae are parallel to

2627 the anteromedial corners, which makes the anterior margin of the supratemporal fenestrae appear

2628 horizontal in dorsal view (state 0 ). This condition is seen in the basal teleosauroid 
2629 Plagiophthalmosuchus (NHMUK PV OR 17892) as well as one teleosauroid subclade (e.g

2630 Macrospondylus MMG BwJ 565; Charitomenosuchus: NHMUK PV R 3320; Proexochokefalos:

2631 MNHN.F 1890-13; Lemmysuchus: NHMUK PV R 3168; Mac. buffetauti: SMNS 91415).

2632 However, in the second subclade, the anterolateral corners of the supratemporal fossae are

2633 noticeably more inclined anteriorly than the anteromedial corners of the supratemporal fossae

2634 (state 1), giving the anterior margin an anteroposteriorly tilted appearance in dorsal view. State 1

2635 is seen in Mystriosaurus (NHMUK PV OR 14781), the Chinese teleosauroid (IVPP V 10098),

2636 Platysuchus (SMNS 9930), Mycterosuchus (NHMUK PV R 2617), Indosinosuchus (PRC-11,

2637 PRC-239) and Aeolodon (MNHN.F.CNJ 78).

2638 104. Supratemporal fenestrae, overall anteroposterior length is either less than or sub-equal to the

2639 anterior width (0), or is twice as long as the anterior width, or more (1) (Fig. 55).

2640 This character is related in part to ch. 102, specifically regarding the parallelogram-

2641 shaped supratemporal fenestrae see in Machimosaurini. In most teleosauroids, the anteroposterior

2642 length of the supratemporal fenestrae is approximately the same as the width (state 0 ). This

2643 condition is in the basal-most form Plagiophthalmosuchus (NHMUK PV OR 14792) as well as

2644 Mystriosaurus (NHMUK PV OR 14781), Indosinosuchus (PRC-11; PRC-239), Platysuchus

2645 (SMNS 9930), Teleosaurus (MNHN AC 8746), Mycterosuchus (NHMUK PV R 2617),

2646 Bathysuchus (unnumbered LPP specimen), Aeolodon (MNHN.F.CNJ 78), Macrospondylus

2647 (MMG BwJ 565), Clovesuurdameredeor (NHMUK PV OR 49126), Charitomenosuchus

2648 (NHMUK PV R 3806) and Deslongchampsina (OUMNH J.29851). In more derived

2649 teleosauroids, the anteroposterior width of the supratemporal fenestrae are approximately twice

2650 as long as the width (state 1). This condition is in Proexochokefalos (MNHN.F 189013), Pr. cf. 
2651 bouchardi (Lepage et al., 2008), Neosteneosaurus (PETMG R178) and machimosaurins (e.g. 2652 Lemmysuchus: NHMUK PV R 3168).

2653 151. The circumorbital dorsal margins of the orbits are flush with the skull dorsal surface (0), 2654 upturned (prominent along the orbital medial margin in dorsal view, with the frontal interorbital 2655 margins being upturned) (1), or upturned along with the posterior margins (the frontal lateral 2656 process anterior margins are also upturned) (2) (Fig. 52).

2657 In the majority of teleosauroids, the orbital dorsal margins are flush (=flattened) with the 2658 skull dorsal surface (state 0) and display no evidence of any dorsal upturn. This condition is seen 2659 in the basal teleosauroid Plagiophthalmosuchus (NHMUK PV OR 14792) as well as 2660 Mystriosaurus (NHMUK PV OR 14781), the Chinese teleosauroid (IVPP V 10098), I. 2661 kalasinensis (PRC-239), Platysuchus (SMNS 9930), Macrospondylus (MMG BwJ 565), 2662 Clovesuurdameredeor (NHMUK PV OR 49126), Charitomenosuchus (NHMUK PV R 3320), 2663 Deslongchampsina (OUMNH J.29851), Proexochokefalos (MNHN.F 1890-13), 2664 Neosteneosaurus (NHMUK PV R 2865) and Machimosaurini (e.g. Lemmysuchus: LPP.M.21).

2665 Four teleosauroid taxa (I. potamosiamensis: PRC-11; Mycterosuchus: NHMUK PV R 2617;

2666 Teleosaurus: MNHN AC 8746; Aeolodon: MNHN.F.CNJ 78) have a definitive upturning of the 2667 orbital dorsal margin (state 1), contributing to the protruding appearance of the orbits.

2668 158. Orbit, the postorbital is excluded from the orbit posteroventral margin or only present in the 2669 posteroventral margin (0), or the postorbital reaches the orbit posteroventral margin and 2670 extensively forms part of the orbit ventral margin (1) (Fig. 56). 
2671 In most teleosauroids, the postorbital does not contact the posteroventral margin of the

2672 orbit (state 0 ). This is the condition seen in the basal-most teleosauroid (Plagiophthalmosuchus:

2673 MNHNL TU515, NHMUK PV OR 14792) as well as more derived taxa (e.g.

2674 Charitomenosuchus: NHMUK PV R 3806; Proexochokefalos: MNHN.F 1890-13;

2675 Yvridiosuchus: OUMNH J.29850; Mac. mosae: IRSNB cast). However, in some teleosauroid

2676 taxa, the postorbital contacts the posteroventral margin of the orbit, forming a substantial

2677 proportion of the orbital ventral margin. Due to this extension, the postorbital often overlaps the

2678 posterior part of the jugal. This condition (state 1) is found in basal teleosauroids (Mystriosaurus:

2679 NHMUK PV OR 14781; the Chinese teleosauroid: IVPP V 10098; I. potamosiamensis: PRC-11;

2680 Platysuchus: SMNS 9930; Teleosaurus: MNHN AC 8746; Mycterosuchus: CAMSM J.1420).

2681 225. Basisphenoid, exposure anterior to the quadrates in palatal view: absent or basisphenoid

2682 terminates approximately level to the anterior extent of the quadrates $(0)$, or basisphenoid

2683 'rostrum' (= cultriform process) is exposed along the palatal surface anterior to the quadrates and 2684 continues to bifurcate the pterygoids (1) (Fig. 57).

2685 In certain teleosauroids, when examining the anterior exposure of the basisphenoid in 2686 palatal view, this bone is either absent or terminates approximately at the level of the anterior2687 most quadrates (state 0). This is the condition seen in the Chinese teleosauroid (IVPP V 10098), 2688 I. potamosiamensis (PRC-11), Teleosaurus (MNHN AC 8746) and Mycterosuchus (CAMSM

2689 J.1420). In the majority of teleosauroids, the basisphenoid is well exposed along the palatal 2690 surface anterior to the quadrates and bifurcates the pterygoids (state 1), which is caused by the 2691 posterior expansion of the posterior margin of the pterygoid. State 1 is a putative synapomorphy 2692 of one teleosauroid subclade and is seen in Macrospondylus (SMNS 81699), 
2693 Clovesuurdameredeor (NHMUK PV OR 49126), Charitomenosuchus (NHMUK PV R 3320),

2694 Deslongchampsina (OUMNH J.29851), Proexochokefalos (MNHN.F 1890-13),

2695 Neosteneosaurus (NHMUK PV R 2865), Yvridiosuchus (OUMNH J.403) and Lemmysuchus

2696 (LPP.M.21).

2697 327. Teeth along the entirety of the tooth row, with sharp, pointed apices $(0)$ or blunt, round 2698 apices (1) (Fig. 40).

2699 Teeth that are elongate and slender with pointed apices (state 0) can clearly be seen in the 2700 basal-most form Plagiophthalmosuchus (MNHNL TU515) and in most teleosauroids (e.g. I.

2701 kalasinensis: PRC-238, PRC-239; Platysuchus: SMNS 9930; Mycterosuchus: NHMUK PV R 2702 2617; Bathysuchus: DORCM G.05067iv; Charitomenosuchus: NHMUK PV 3806). While the 2703 taxa Mystriosaurus (HLMD V946-948, NHMUK PV OR 14781), Proexochokefalos (MNHN.F

2704 1890-13), Deslongchampsina (OUMNH J.29851) and Neosteneosaurus (PETMG R178) possess

2705 teeth with pointed apices (and are therefore scored as state 0), it is important to note that the 2706 overall dentition of these four genera are more robust than in the other aforementioned 2707 teleosauroids. In particular, the posterior teeth of Neosteneosaurus (PETMG R178) are 2708 noticeably more conical but continue to retain a pointed apex. The tribe Machimosaurini (Jouve 2709 et al., 2016) is unique in that all members (Yvridiosuchus: OUMNH J.29850; Lemmysuchus:

2710 NHMUK PV R 3618; Machimosaurus: LMH 16387, LMH 16405, MG-8730-1, ONM NG 7, 2711 SMF 2027, SMNS 91415) have conical teeth with blunt, rounded apices (state 1) throughout the 2712 entirety of the dentition.

2713 358. Morphology of apical enamel surface ornamentation, macroscopic anastomosed pattern 2714 absent (0) or present (1) (Fig. 40). 
2716 unornamented aside from the enamel ridges that reach the tip of the apex (state 0 ) in most

2717 teleosauroids. This is the condition seen in Plagiophthalmosuchus (MNHNL TU515), as well as

2718 Mystriosaurus (NHMUK PV OR 14781); I. kalasinensis (PRC-239); Platysuchus (SMNS 9930);

2719 Teleosaurus (Eudes-Deslongchamps, 1867-69); Mycterosuchus (NHMUK PV R 2617);

2720 Bathysuchus (DORCM G.05067iv); Sericodon (TCH005-151 in Schaefer, Püntener \& Billon-

2721 Bruyat, 2018); Aeolodon (NHMUK PV R 1086); Macrospondylus (MNHNL TU799);

2722 Charitomenosuchus (NHMUK PV R 3806); Seldsienean (OUMNH J.1414); Deslongchampsina

2723 (OUMNH J.29851); Proexochokefalos (MNHN.F 1890-13); and Neosteneosaurus (NHMUK PV

2724 R 3701; PETMG R178). However, the tribe Machimosaurini evolved a complex ornamentation

2725 pattern (state 1); this pattern is often referred to as 'anastomosed', which is a rough, 'wrinkled'

2726 texture, visible to the naked eye, on the apical third of the tooth. Anastomosed teeth are one of

2727 the characteristic features in machimosaurins, present in all members of the group

2728 (Yvridiosuchus: OUMNH J.29850; Lemmysuchus: NHMUK PV R 3168; Machimosaurus:

2729 SMNS 91415, MG-8730-1, ONM NG 7, SMF 2027).

2730 379. Number of sacral vertebrae: two (0) or three (1) (Fig. 43).

2731 In the majority of teleosauroids, there are two sacral vertebrae (state 0 ). This condition is 2732 seen in the basal form Plagiophthalmosuchus (NHMUK PV OR 14792) as well as Platysuchus 2733 (SMNS 9930), Teleosaurus (NHMUK PV OR 32588), Mycterosuchus (NHMUK PV R 2617), 2734 Aeolodon (MNHN.F.CNJ 78), Macrospondylus (SMNS 52034), Charitomenosuchus (NHMK 2735 PV R 3806), and Neosteneosaurus (NHMUK PV R 3701, PETMG R178). However, in scored 2736 members of Machimosaurini (Lemmysuchus: NHMUK PV R 3618; Mac. mosae: IRSNB cast, 
2737 Hua, 1999), three sacral vertebrae are present (state 1), which is a unique feature of this clade.

2738 The first two vertebrae are true sacrals, with the first caudal vertebra appearing and functioning 2739 as a third sacral.

2740

2741 410. Humerus, humeral head: confined to the proximal surface $(0)$, gently posteriorly expanded 2742 and hooked (1), or very strongly posteriorly deflected and hooked (2) (Fig. 58).

2744 expanded and hooked (state 1) or strongly deflected and hooked (state 2); it is never confined to

2745 the proximal surface (state 0). In basal teleosauroids such as Plagiophthalmosuchus (NHMUK

2746 PV OR 14792), Platysuchus (SMNS 9930), Teleosaurus (OUMNH J.26801), Macrospondylus

2747 (SMNS 51957) and Mycterosuchus (NHMUK PV R 2617), the proximal humerus (or humeral

2748 head) is anteroposteriorly elongated and gently but noticeably hooked (state 1). In the

2749 teleosauroids Aeolodon (MNHN.F.CNJ 78), Charitomenosuchus (NHMUK P R 3806) and

2750 Neosteneosaurus (PETMG R178), the posterior deflection of the proximal humerus is strong, so

2751 much so that the proximal epiphysis is noticeably posterior to the distal epiphysis. This posterior

2752 deflection is much more pronounced than in any other thalattosuchian taxa.

2753 420. Ulna, olecranon process mediolaterally compressed and greatly proximally expanded: no

2754 (0), yes (1) (Fig. 44).

2755 Only two basal teleosauroids (Platysuchus: SMNS 9930; Macrospondylus SMNS 53422)

2756 score as 0 , in which the olecranon process is neither compressed nor expanded. Interestingly, 2757 more derived teleosauroids score as state 1, where the olecranon process is both greatly expanded 
2758 and mediolaterally compressed. This is seen in Mycterosuchus (NHMUK PV R 2617), Aeolodon

2759 (MNHN.F.CNJ 78), Charitomenosuchus (NHMUK PV R 3806), Neosteneosaurus (PETMG

2760 R178) and Lemmysuchus (NHMUK PV R 3168).

2761 440. Ilium, postacetabular (= posterior) process expanded into a thin 'fan' shape: no (0), yes (1) 2762 (Fig. 46).

2763 In most teleosauroids, the postacetabular (=posterior) iliac process is either

2764 anteroposteriorly shortened, robust and process-like (state 0 ) or anteroposteriorly expanded and

2765 mediolaterally thin, expanding it into a 'fanlike' shape (state 1), and is best seen in either lateral

2766 or medial view. In Charitomenosuchus (NHMUK PV R 3806), Neosteneosaurus (PETMG

2767 R178), Lemmysuchus (NHMUK PV R 3816) and Mac. mosae (Young et al., 2014), state 1 is

2768 present, with the postacetabular process lengthened into a mediolaterally thin 'fan-like' shape.

2769 However, it is important to note that state 1 is a putative apomorphy of derived teleosauroids, and 2770 is not seen in basal taxa such as Plagiophthalmosuchus (NHMUK PV OR 14792), Platysuchus

2771 (SMNS 9930), Teleosaurus (NHMUK PV OR 32588), Sericodon (SCR010-312 in Schaefer,

2772 Püntener \& Billon-Bruyat, 2018) and Macrospondylus (SMNS 18672, SMNS 51753).

2773 473. Ornamentation (dorsal osteoderms), the pits are either small round to ellipsoid and very

2774 densely distributed (0), large round to ellipsoid and well separated (1), irregularly shaped with an

2775 extreme variation in size, with elongate pits present on the ventrolateral surface running from the

2776 keel to the lateral margin (2), or variable in both size, shape and length that radiate in a starburst 2777 pattern (3) (Fig. 51). 
across taxa, the ornamentation (or pitting) pattern differs, most notably in the thoracic/sacral

2780

osteoderms. In most teleosauroids, the pits are large, subcircular to ellipsoid in shape, and

2781 generally well separated from one another. This condition (state 1) is seen in

2782

Plagiophthalmosuchus (NHMUK PV OR 14792), Mycterosuchus (NHMUK PV R 2617),

2783 Charitomenosuchus (NHMUK PV R 3806) and Neosteneosaurus (NHMUK PV R 2865;

2784 NHMUK PV R 3701; PETMG R178). In Charitomenosuchus (NHMUK PV R 3806), the pits are

2785 arranged in a semi-circular pattern, and the larger ones are situated more towards the lateral

2786 margins of the osteoderm. In Neosteneosaurus (NHMUK PV R 2865), most pits are

2787 exceptionally large (especially situated in the centre of the osteoderm), subcircular and fewer in

2788 number. While the osteoderm ornamentation in the holotype of Macrospondylus (MMG BwJ

2789 595) is poorly preserved, the pits appear to be large and semi-ellipsoid with a strong

2790 anteroposterior keel. The pits also appear to be more closely placed to one another, which is

2791 observed in other Macrospondylus specimens (e.g. MMG BwJ 565; SMNS 51563; SMNS

2792 51753), with a thin ridge separating them. In two teleosauroid taxa, the ornamental pits are small,

2793 round, and extremely densely distributed throughout the entirety of the dorsal osteoderms (state

2794 0). This is seen in Platysuchus (SMNS 9930) and Teleosaurus (NHMUK PV R 119a). Certain

2795 teleosauroids, however, possess thoracic/sacral osteoderms with exceptionally enlarged,

2796 elongated pits; due to this elongation and large size, these pits merge with one another and

2797 become elongated grooves, especially along the lateral margins, with the pits radiating distally in

2798 a 'starburst' pattern (state 3). The remainder of the pits are variable in size (from small to large),

2799 irregularly shaped, and relatively close together. In addition, well-developed keels are generally

2800 present in these osteoderms. This condition is observed in machimosaurins (Lemmysuchus: 
2801 NHMUK PV R 3618; Machimosaurus: ONM 1-25, SMNS 91415, Young et al., 2014). State 2,

2802 in which the pits are all irregularly shaped with extreme variation in size and have no 'starburst'

2803 pattern, is not present in any known teleosauroid taxa.

2804

2805 Cladistic Analysis: Results

2806

1.1 Most parsimonious unweighted strict consensus

2807 The initial New Technology search recovered 125 most parsimonious trees (MPTs) of 1659 steps

2808 (ensemble consistency index $(\mathrm{CI})=0.405$; ensemble retention index $(\mathrm{RI})=0.844$; ensemble

2809 rescaled consistency index $(\mathrm{RCI})=0.342$; ensemble homoplasy index $(\mathrm{HI})=0.595)($ Fig. 59A).

2810 With TBR branch swapping set to 100, 260 MPTs and 1659 steps were recovered; when set to

2811 1000, 2740 MPTs and 1659 steps were found, with the best score hitting 301 out of 1000 times.

2812 The overall topology did not change, with or without TBR.

2813 In this topology, Eopneumatosuchus colberti Crompton and Smith, 1980, was found to be

2814 the immediate outgroup to Thalattosuchia, which was divided into two groups:

2815 Metriorhynchoidea and Teleosauroidea. Within Teleosauroidea, Plagiophthalmosuchus was

2816 recovered as the basal-most teleosauroid. This is weakly supported, with a jackknife percentage

2817 of $66 \%$ and a Bremer support value of 1 . There are two main teleosauroid families recovered (see

2818 discussion on clades below), with the taxa Clovesuurdameredeor and Macrospondylus (which

2819 form a separate polytomy) being most closely related to both of them. Within the first family

2820 (Family T) (Fig. 59A), I. kalasinensis, I. potamosiamensis, the Chinese teleosauroid (IVPP V

2821 10098) and Mystriosaurus are unresolved with one another and are most closely related to two

Peer) reviewing PDF | (2020:03:47227:2:0:NEW 29 Jul 2020) 
2822 remaining subfamilies (see below). The taxa Teleosaurus and Platysuchus are each other's

2823 closest relatives, with a Bremer support value of 2 and jackknife percentage of 54\%.

2824 Interestingly, Mycterosuchus, Aeolodon, Bathysuchus and Sericodon form a distinct subfamily.

2825 Bathysuchus and Sericodon are sister taxa (Bremer support value of 3 and jackknife of 88\%);

2826 Aeolodon is most closely related to Sericodon+Bathysuchus, and Mycterosuchus is most closely

2827 related to Aeolodon+Bathysuchus + Sericodon.

2828

Within the second family (Family M) (Fig. 59A), there are multiple unresolved areas.

2829 Seldsienean, Deslongchampsina and Charitomenosuchus are unresolved from one another and

2830 are situated at the base of this clade (Bremer support value of 1 and jackknife of 66\%). Most

2831 notably, there is a large polytomy including Pr. heberti, Pr. cf. bouchardi, Neosteneosaurus, $S$.

2832 rostromajor, Andrianavoay, Lemmysuchus and Yvridiosuchus, and Machimosaurini is not

2833 recovered as a monophyletic subgroup. However, when S. rostromajor is removed from the

2834 analysis (176 MPTs and 1659 steps: $\mathrm{CI}=0.405, \mathrm{RI}=0.844)$, Machimosaurini becomes a distinct

2835 group, with Lemmysuchus + Yvridiosuchus and Machimosaurus separated from Neosteneosaurus,

2836 Pr. heberti, Pr. cf. bouchardi and Andrianavoay (Fig. 59B). In addition, when both $S$.

2837 rostromajor and Andrianavoay are removed (167 MPTs, 1659 steps: $\mathrm{CI}=0.405, \mathrm{RI}=0.844), \operatorname{Pr}$.

2838 heberti and Pr. cf. bouchardi are unresolved from one another but separated from

2839 Neosteneosaurus, which by itself becomes most closely related to Machimosaurini. In all

2840 iterations (with or without the removal of S. rostromajor and Andrianavoay), the genus

2841 Machimosaurus forms its own subgroup, and relationships between the four species are mostly

2842 resolved. Machimosaurus mosae and Mac. buffetauti are unresolved from one another; and Mac.

2843 rex and Mac. hugii are sister taxa (with Mac. mosae+Mac. buffetauti being most closely related

2844 to them). 
28451.2 Most parsimonious unweighted consensus - majority rules

2846 A parsimonious majority rules topology was produced to evaluate if there were any major

2847 changes from the strict consensus. The overall interrelationships within Teleosauroidea are more

2848 resolved than in the strict consensus topology (Fig. 59C), particularly within Family M. In

2849 Family T (Fig. 59C), I. kalasinensis is most closely related to the remaining taxa, and $I$.

2850 potamosiamensis and the Chinese teleosauroid (IVPP V 10098) are sister taxa, with

2851 Mystriosaurus being most closely related to them.

2852 In Family M (Fig. 59C), Clovesuurdameredeor is situated at the base of this group, in

2853 stark contrast to its initial positioning, and Deslongchampsina, Charitomenosuchus and

2854 Seldsienean are all separated. A new subfamily (consisting of Pr. heberti, Pr. cf. bouchardi,

2855 Andrianavoay, Neosteneosaurus, S. rostromajor and Machimosaurini) is clearly defined (100\%),

2856 and Deslongchampsina is most closely related to this subfamily. Proexochokefalos heberti is

2857 most closely related to $P r$. cf. bouchardi+Neosteneosaurus $+S$.

2858 rostromajor+Andrianavoay+Machimosaurini. Proexochokefalos $\mathrm{cf}$. bouchardi,

2859 Neosteneosaurus, S. rostromajor and Andrianavoay are all unresolved from one another, and are

2860 most closely related to Machimosaurini. Unlike the strict consensus topology (when all taxa are

2861 included), Machimosaurini is relatively well-supported (73\%); Lemmysuchus and Yvridiosuchus

2862 (unresolved from one another) are separate from Andrianavoay, Neosteneosaurus and S.

2863 rostromajor, and are at the base of Machimosaurini. Machimosaurus buffetauti and Mac. mosae

2864 are separated, with Mac. mosae being the more closely related to Mac. rex and Mac. hugii

2865 (which are sister taxa) than Mac. buffetauti. It is important to note that when S. rostromajor is

2866 removed from the majority rules consensus, there is no change to teleosauroid interrelationships. 
2867 1.3 Most parsimonious weighted strict consensus

2868 As outlined above, the analysis was run once more using extended implied weights $(\mathrm{k}=12)$.

2869 Extended implied weights (EIWs) are often used to improve the quality and stability of the 2870 results, and are more beneficial for palaeontological datasets than implied weights, which only

2871 introduces bias against characters with too many missing scores (Goloboff, 2014). The New

2872 Technology search (engines tailored as above) with TBR branch swapping resulted in 47 MPTs 2873 and a score of 48.94448 . Due to relative clarity in the results, this is the topology referred to 2874 when formally naming clades (see below). The results of the EIW analysis (Fig. 60A) show a more resolved Teleosauroidea than in

2876 the original strict consensus and is more similar regarding the majority rules topology.

2877 Teleosauroidea is monophyletic, Plagiophthalmosuchus is the basal-most teleosauroid, and the 2878 two families $\mathrm{T}$ and $\mathrm{M}$ are recovered. Family $\mathrm{T}$ is fully resolved (Fig. 60A), in contrast to both 2879 unweighted consensus topologies. Firstly, the Chinese teleosauroid (IVPP V 10098) and 2880 Mystriosaurus form sister taxa (although, surprisingly, there are no unambiguous 2881 synapomorphies to support this), with I. kalasinensis (situated at the base of this clade) being 2882 most closely related to them; in the majority rules topology, I. potamosiamensis was the sister 2883 taxon to the Chinese teleosauroid (IVPP V 10098). Here, I. potamosiamensis is positioned as 2884 most closely related to the Teleosaurus + Platysuchus subclade and subclade composed of 2885 Mycterosuchus + Aeolodon+Bathysuchus + Sericodon. Teleosaurus and Platysuchus are once again 2886 sister taxa, and they are most closely related to Mycterosuchus and pelagic relatives, which 2887 differs from the majority rules topology. The positioning of Mycterosuchus, Aeolodon, Sericodon 2888 and Bathysuchus are the same as all previous results: 
2889

2890

2891

2892

2893 the majority rules topology:

2894

2895

2896

2897

2898

2899

2900

2901

2902 2903 2904 2905 2906 2907 2908 2909 clade; and

1. Macrospondylus, rather than Clovesuurdameredeor, is the basal-most member of this

2. Notably, and surprisingly, Machimosaurini is not found to be monophyletic, with Lemmysuchus and Yvridiosuchus forming a polytomy with Neosteneosaurus, $S$. rostromajor and Andrianavoay. This is similar to the original consensus rather than the majority rules topology.

Deslongchampsina is once again found to be most closely related to the subfamily containing Pr. heberti, Pr. cf. bouchardi, S. rostromajor, Andrianavoay and Machimosaurini. Proexochokefalos cf. bouchardi and Pr. heberti are sister taxa, as in the majority rules topology. When S. rostromajor is removed, (Fig. 60B), the only change results in Machimosaurini being consistently recovered, as Yvridiosuchus and Lemmysuchus are separated from Neosteneosaurus and Andrianavoay. Interrelationships within Machimosaurus taxa were identical to the majority rules topology: Mac. hugii and Mac. rex are sister taxa, and Mac. mosae is most closely related to Mac. hugii+Mac. rex than Mac. buffetauti.

There are possible explanations as to why the tribe Machimosaurini remains unresolved from certain non-machimosaurins when all taxa are included. Firstly, both S. rostromajor and 
2910 Andrianavoay are both represented by fragmentary skull material (and therefore scored for a low

2911 amount of characters), which may contribute to the lack of resolution. Another crucial factor is

2912 the lack of postcranial material for Andrianavoay, S. rostromajor and Yvridiosuchus;

2913 machimosaurins have a very distinct postcranium (e.g. Hua, 1999; Young et al., 2014; Johnson et

2914 al., 2017), which may influence the appearance of the topology. Thirdly, there are no

2915 autapomorphies observed in S. rostromajor, which is a poorly preserved section of undiagnostic

2916 rostrum (see Johnson, Young \& Brusatte, 2020, for more information). This may contribute to

2917 the uncertainty of its placement as either an intermediate non-machimosaurin (e.g.

2918 Neosteneosaurus) or basal machimosaurin (e.g. Yvridiosuchus).

2919 1.4 Agreement subtree

2920 The maximum agreement subtree (which chooses a subset of species with an equivalent

2921 restricted tree in all given evolutionary circumstances; Amir \& Keselman, 1997), for

2922 Teleosauroidea was also produced (Fig. 60C) from the unweighted strict consensus:

2923 Plagiophthalmosuchus was recovered as the basal-most teleosauroid, and Families T and M were

2924 resolved. In Family T, Teleosaurus +Platysuchus and

2925 Mycterosuchus + Bathysuchus + Aeolodon+Sericodon were recovered as monophyletic subclades.

2926 In Family M, Macrospondylus was situated at the base and Deslongchampsina was most closely

2927 related to Pr. cf. bouchardi + Neosteneosaurus + Machimosaurini. Surprisingly, Pr. cf.

2928 bouchardi was recovered at most closely related to Neosteneosaurus + Machimosaurini.

2929 Machimosaurus rex and Mac. hugii were also recovered as sister taxa, and Mac. buffetauti was

2930 most closely related to them. Lemmysuchus was situated at the base of Machimosaurini, with

2931 Neosteneosaurus as the closest relative. Therefore, the taxa identified as hypothetically

Peer) reviewing PDF | (2020:03:47227:2:0:NEW 29 Jul 2020) 
2932 responsible for poor resolution (not included in the agreement tree) were Indosinosuchus,

2933 Mystriosaurus, the Chinese teleosauroid, Clovesuurdameredeor, Charitomenosuchus,

2934 Seldsienean, S. rostromajor, Andrianavoay, Pr. heberti, Yvridiosuchus and Mac. mosae. This is

2935 logical, as most aforementioned taxa either are fragmentary, lack postcrania or are represented

2936 by a low number of specimens (excluding Charitomenosuchus). As mentioned previously, these

2937 are key factors that can lead to polytomies and lack of resolution in trees. However, it is

2938 interesting to note that Pr. cf. bouchardi is included in the agreement subtree as a stable taxon,

2939 even though it is a partial skull scored based off specimen photographs.

29401.5 Bayesian results

2941 As mentioned previously, three repetitions of MrBayes were run using the following functions:

2942 (\#1) standard (rates=equal); (\#2), gamma distribution (rates=gamma); and (\#3) gamma

2943 distribution with variability (1set applyto=(1) coding=variable $)$. The standard Bayesian results

2944 (\#1) are relatively similar to those found in the implied weighting parsimony topology (standard

2945 deviation $=0.015520$; harmonic mean $=-8131.53$ ). Teleosauroidea is monophyletic,

2946 Plagiophthalmosuchus is the basal-most teleosauroid and both Families T and M are recovered.

2947 However, there are slight differences within both subclades. In Family T, Platysuchus and

2948 Teleosaurus (sister taxa) are unresolved with Mycterosuchus+relatives and the East Asian

2949 teleosauroids + Mystriosaurus, and the East Asian teleosauroids (much like in the strict consensus

2950 and majority rules topologies), and I. potamosiamensis is most closely related to the Chinese

2951 teleosauroid+Mystriosaurus. In Family M, Pr. cf. bouchardi and Pr. heberti are not sister taxa,

2952 but rather $P r$. cf. bouchardi is found to be most closely related to

2953 Neosteneosaurus + Andrianavoay + S. rostromajor + Machimosaurini. 
2954 In the gamma Bayesian test $(\# 2)$, the results (standard deviation $=0.019863$; harmonic 2955 mean $=$-7785.47) (Fig. 61) are similar to that seen in the standard Bayesian analysis, but with 2956 two differences:

2957

2958

2959

2960

2961

2962

\section{Clades and their synapomorphies}

2964 Within this section, the synapomorphies uniting major clades are highlighted and discussed. A 2965 2966 2967 2968 2969 2970

2971 Classification note. Teleosauroidea is a 'family group' clade established under the ICZN Code, 2972 at the superfamily rank. 
2973 Nominal authority. The nominal authority is based on Article 36.1 of the ICZN Code (Principal 2974 of Coordination, applied to family group names).

2975 Description. The superfamily Teleosauroidea is supported by multiple synapomorphies. These 2976 include absence of a sclerotic ring (163.0), postorbital medial to the jugal on the postorbital bar 2977 (173.0), straightened (sub-rectangular) anterior maxilla in palatal view (184.1), relatively 2978 reduced occipital tuberosities (203.1), paired ridges located on the medial ventral surface of the 2979 basisphenoid (223.1), a distinctly spatulate anterior dentary with the maximum width at the D32980 D4 couplet (254.2), D3 occludes against the premaxillary-maxillary suture (331.0), coracoid with 2981 a fan-shape distal end and a triangular-shaped proximal end (402.1), a scapular blade as wide as 2982 or narrower than the glenoid region (405.1) and presence of caudal armour (493.0), as well as 2983 scoring the 'pholidosaurid beak' as inapplicable (47.-). One of these characters is new to the 2984 dataset, and another character (47) was re-written and re-scored. It is important to note that in 2985 teleosauroids, certain characters score differently than Pelagosaurus but are the same for other 2986 basal metriorhynchoids (e.g. Teleidosaurus). These include a slightly convex or flat frontal 2987 (121.0), a broadly curved anterior margin of the external mandibular fenestra (260.0), and well2988 defined apicobasally aligned ornamental ridges on the dentition (357.4),

2989 Comments. Geoffroy Saint-Hilaire (1831: 34) initially defined teleosauroids (interpreted as 2990 'Teleosauridae') as a distinct clade, referring to "un cachet crocodilien" ("a crocodilian 2991 character"). This suggests that he is describing the main features of teleosauroids, although he 2992 did not assign a name to this clade (Johnson, Young \& Brusatte, 2020). He then proceeds to list 2993 the following features as definitive for the group:

1. Large 'vertical holes' (supratemporal fenestrae); 
2995 2. Vertically placed eyes;

2996 3. A parietal bone that does not intervene between the jugal and temporal;

2997 4. Two arches (“l'une supérieure jugo-temporale, l'autre inférieure maxillo-tympanique":

2998 "one superior jugo-temporal, the other lower maxillofacial");

2999 5. Development of the nasal (cranio-respiratory) canal and temporal region; and

3000 6. 'Beak-like' snout.

3002 dernière combinaison remarquable dans les êtres téléosauriens devient des éléments

3003 caractéristiques pour une nouvelle famille; des éléments d'une puissance et d'une valeur à

3004 rendre en effet obligatoires les distinctions zoologiques de cette famille, c'est-à-dire l'érection

3005 des genres Téléosaurus et Sténéosaurus" ("This last remarkable combination in teleosaurs

3006 becomes characteristic elements for a new family; elements of power and value to make

3007 compulsory the zoological distinctions of this family, that is to say the erection of the genera

3008 Teleosaurus and Steneosaurus"). Geoffroy Saint-Hilaire (1831: 37) considered "la région

3009 supérieure et vers la fin de l'arrière-crâne; et d'autre part le museau" ("the upper region and

3010 towards the end of the back of the skull; and [on the other hand] the snout"), along with "le canal

3011 nasal et le palais" ("the nasal canal and the palate"), to be the most important features when

3012 distinguishing teleosauroid species. After Geoffroy Saint-Hilaire's (1831) work, teleosauroids

3013 continued to be traditionally grouped together based on their 'longirostrine' skull, dorsally

3014 directed orbits and high tooth count (Karl et al., 2008; Young \& Andrade, 2009; Ballell et al.,

3015 2019). However, recent studies (e.g. Young et al., 2014; Foffa et al., 2019; Sachs et al., 2019a)

3016 have shown that there is more variation in the teleosauroid cranium than initially thought, and the

3017 shape of the skull and number of teeth cannot purely be relied on to define this clade. 
3019 Registration number. To be added in proof.

3020 Phylogenetic definition. The largest clade within Thalattosuchia containing Teleosaurus

3021 cadomensis, but not Metriorhynchus geoffroyii von Meyer, 1832. Young \& Andrade (2009)

3022 initially defined the superfamily Teleosauroidea as the most inclusive clade consisting of

3023 Teleosaurus cadomensis, but not Metriorhynchus geoffroyii. This is a maximum-clade, or stem3024 based, definition.

3025 Reference phylogeny. Phylogenetic analyses presented herein, see figures 59-61 and 63-64.

3026 Composition. Plagiophthalmosuchus, Teleosauridae (comprising of the Chinese teleosauroid, 3027 Indosinosuchus, Mystriosaurus, Teleosaurinae [comprising of Platysuchus and Teleosaurus], 3028 and Aeolodontinae [comprising of Aeolodon, Bathysuchus, Mycterosuchus and Sericodon] and 3029 Machimosauridae (comprising of Charitomenosuchus, Clovesuurdameredeor, 3030 Deslongchampsina, Macrospondylus, Seldsienean and Machimosaurinae [comprising 3031 Andrianavoay, Neosteneosaurus, Proexochokefalos and Machimosaurini \{comprising 3032 Lemmysuchus, Machimosaurus and Yvridiosuchus\}]).

3033 Diagnostic apomorphies. 47.-; 163.0; 173.0; 184.1; 203.1; 223.1; 254.2; 331.0; 402.1; 405.1;

3034 493.0. (From the dataset herein, the same characters as the ICZN Code description.)

3036 Classification note. Teleosauridae is a 'family group' clade established under the ICZN Code, at 3037 the family rank. 
3038 Original Definition Comment. 'Teleosauridae' was originally erected and defined by Geoffroy

3039 Saint-Hilaire $(1825,1831)$ and encompassed all teleosauroid species (as discussed above).

3040 However, herein Teleosauridae is restricted to the following taxa: the genus Indosinosuchus,

3041 Mystriosaurus laurillardi, Teleosaurus cadomensis, Platysuchus multiscrobiculatus, Aeolodon

3042 priscus, Mycterosuchus nasutus, Sericodon jugleri, Bathysuchus megarhinus and the Chinese

3043 teleosauroid (IVPP V 10098).

3044 Description. A number of synapomorphies supports the monophyly of Teleosauridae. These

3045 include anteriorly or anterodorsally oriented external nares (34.0), anterior and anterolateral

3046 premaxillary margins that are anteroventral and extend ventrally (48.1), supratemporal fenestrae

3047 with noticeably inclined anterior margins (103.1), postorbital overlapping the jugal (158.1), a

3048 horizontal pterygoid flange (198.0) and the basisphenoid terminates at the anterior quadrates

3049 (225.0).

3050

Teleosauridae Geoffroy Saint-Hilaire, 1831 nomen cladi conversum

3051 Registration number. To be added in proof.

3052 Phylogenetic definition. The largest clade within Teleosauroidea containing Teleosaurus

3053 cadomensis, but not Plagiophthalmosuchus gracilirostris and Machimosaurus hugii. This is a

3054 maximum-clade, or stem-based, definition.

3055 Reference phylogeny. Phylogenetic analyses presented herein, see figures 59-61 and 63-64. 
3056 Composition. The Chinese teleosauroid, Indosinosuchus, Mystriosaurus, Teleosaurinae

3057 (comprising of Platysuchus and Teleosaurus), and Aeolodontinae (comprising of Aeolodon, 3058 Bathysuchus, Mycterosuchus and Sericodon).

3059 Diagnostic apomorphies. $34.0 ; 48.1 ; 103.1 ; 158.1 ; 198.0 ; 225.0$. (From the dataset herein, the 3060 same characters as the ICZN Code description.)

3062 Comments. Interestingly, there are no unambiguous synapomorphies that unite this clade, 3063 despite its stable position within the weighted parsimonious analysis (Fig. 60A-B). This 3064 unnamed clade shares one character with Neosteneosaurus and machimosaurins (nasals and 3065 maxillae are not elongated: 6.0) and one character with Mac. buffetauti and Mac. mosae 3066 (anteroposterior premaxillary length is less than 25\% of total rostrum length: 43.0).

3068 Classification note. Teleosaurinae is a 'family group' clade established under the ICZN Code, at 3069 the subfamily rank.

3070 Nominal authority. The nominal authority is based on Article 36.1 of the ICZN Code (Principal 3071 of Coordination, applied to family group names).

3072 Description. The subfamily Teleosaurinae consists of the genera Platysuchus and Teleosaurus, 3073 and there are four characters that unite them as sister taxa. These include both the tooth row and 3074 quadrate condyle being below the level of the occipital condyle but are unaligned with the tooth 3075 row at a lower level (2.5), the frontal-postorbital suture is lower than the intertemporal bar 
3076 (131.1), densely distributed osteoderms with small round to ellipsoid pits (473.0), and presacral 3077 dorsal osteoderms are strongly curved (480.1).

3078 Comments. Vignaud (1995) initially diagnosed the subfamily Teleosaurinae as that containing 3079 Platysuchus and all Teleosaurus taxa. Here, Teleosaurus is currently limited to just one species, 3080 but follows the same proposal put forth in Vignaud (1995), in that Platysuchus is most closely 3081 related to Teleosaurus.

3082 Teleosaurinae Vignaud, 1995 nomen cladi conversum

3083 Registration number. To be added in proof.

3084 Phylogenetic definition. The largest clade within Teleosauroidea containing Teleosaurus 3085 cadomensis, but not Aeolodon priscus and Indosinosuchus potamosiamensis. This is a maximum3086 clade, or stem-based, definition.

3087 Reference phylogeny. Phylogenetic analyses presented herein, see figures 59-61 and 63-64.

3088 Composition. Platysuchus and Teleosaurus.

3089 Diagnostic apomorphies. 2.5; 131.1; 473.0; 480.1. (From the dataset herein, the same characters 3090 as the ICZN Code description.) Aeolodontinae subfam. nov. (Mycterosuchus + Aeolodon + Bathysuchus + Sericodon)

3092 Classification note. Aeolodontidae is a 'family group' clade established under the ICZN Code, 3093 at the subfamily rank. urn:lsid:zoobank.org:act:E7A8EDC8-8DF8-4799-AA09-5D6B9287E201. 
3094 Description. A number of synapomorphies, notably in the premaxilla, supports the subfamily

3095 Aeolodontinae, which includes the genera Mycterosuchus, Aeolodon, Sericodon and

3096 Bathysuchus. These include an '8'shaped premaxilla in anterior view (56.1), reduced

3097 basioccipital tuberosities (230.0), laterally oriented P1 and P2 (294.2), P1 and P2 do not form a

3098 couplet but are situated on the anterior margin of the premaxilla (295.1), P1 and P2 are both on

3099 the same transverse plane (298.1) and the anterior margin between the P2-P3 is sub-rectangular,

3100 with the P3 being clearly lateral to the P2 (299.1). Four out of six characters are new to this

3101 dataset.

3102 Comments. Aeolodontinae is also always recovered as a monophyletic subclade, regardless of

3103 changing taxa and/or character scores and whether the dataset is run using parsimony or

3104 Bayesian criteria. It is interesting to note that, while similar in many aspects concerning the skull

3105 (namely the premaxillae), the postcranial material of Mycterosuchus differentiates vastly from

3106 other members of the group. For example, the proximal humerus is very strongly posteriorly

3107 deflected and hooked in Aeolodon, similar to members of Machimosauridae (e.g.

3108 Charitomenosuchus, Neosteneosaurus). In Mycterosuchus, the proximal humerus is also hooked,

3109 but weakly so, and is more club-shaped. The tuberculum and articular facet of the largest dorsal

3110 ribs are positioned directly in the middle, which is more similar to Charitomenosuchus and

3111 opposed to the medial edge position in Aeolodon. Other unique postcranial features to

3112 Mycterosuchus include a longer ulna than radius, an elongated pubic shaft, an enlarged

3113 anteromedial femoral tuber and the calcaneal tuber being approximately $25 \%$ larger than the

3114 astragalus (as discussed above). It is likely that the unique skull characteristics of these taxa are

3115 what is supporting this subfamily as monophyletic. 
3117 PV R 1086 and MNHN.F.CNJ 78), and partially preserved in Sericodon (see Schaefer, Püntener

3118 \& Billon-Bruyat, 2018), it is important to note that there are no postcranial bones of Bathysuchus

3119 currently recorded. A full, comprehensive comparison of the postcrania of Aeolodon and

3120 Sericodon is essential, to examine if Sericodon possesses a reduced appendicular skeleton similar

3121 to that seen in Aeolodon, which has been hypothesized to be more pelagic than other

3122 teleosauroids (see below, as well as Foffa et al. [2019]).

3123

\section{Aeolodontinae nomen cladi novum}

3124 Registration number. To be added in proof.

3125 Phylogenetic definition. The largest clade within Teleosauroidea containing Aeolodon priscus 3126 but not Indosinosuchus potamosiamensis and Teleosaurus cadomensis. This is a maximum3127 clade, or stem-based, definition.

3128 Reference phylogeny. Phylogenetic analyses presented herein, see figures 59-61 and 63-64.

3129 Composition. Aeolodon, Bathysuchus, Mycterosuchus and Sericodon.

3130 Diagnostic apomorphies. 56.1; 230.0; 294.2; 295.1; 298.1; 299.1. (From the dataset herein, the 3131 same characters as the ICZN Code description.)

\section{Unnamed clade: Aeolodon + Bathysuchus + Sericodon}

3133 Comments. Interestingly, there are no unambiguous synapomorphies that unite this clade,

3134 despite its stable position within the above analyses. This unnamed clade shares two characters 
3135 with Plagiophthalmosuchus and I. potamosiamensis: no ornamentation on prefrontal (12.1) and

3136 lacrimal (13.1); and one character with Charitomenosuchus, Seldsienean, Deslongchampsina and

3137 Machimosaurinae (see below): frontal ornamentation restricted to the centre of the bone (15.1).

3138

\section{Unnamed clade: Sericodon + Bathysuchus}

3139 Synapomorphies. 296.1; 339.1.

3140 Comments. Sericodon and Bathysuchus are united by two characters: a strong lateral expansion

3141 of the premaxillae so that P3 and P4 are aligned on the lateral plane of the external margin

3142 (296.1) and presence of carinae on the apical third of the tooth (339.1). Despite only two dental

3143 synapomorphies, Sericodon and Bathysuchus are recovered as sister taxa in all analyses.

\section{Machimosauridae Jouve et al., 2016 fam. nov. (Family M)}

3145 Classification note. Machimosauridae is a 'family group' clade established under the ICZN

3146 Code, at the family rank. urn:1sid:zoobank.org:act:81FB2470-D7E7-4E3E-814B-

3147 2AAE996BE5AA.

3148 Nominal authority. The nominal authority is based on Article 36.1 of the ICZN Code (Principal 3149 of Coordination, applied to family group names).

3150 Description. The family Machimosauridae is united by a number of characters; these include the

3151 dorsally oriented external nares (34.1), the premaxillary anterior and anterolateral margins are

3152 not sub-vertical and do not extend ventrally (48.0), the premaxilla-maxilla suture is sub-

3153 rectangular and slightly interdigitating (most noticeably near the midline) (58.1), no anterolateral

3154 expansion of the supratemporal fenestrae (103.0), the postorbital excluded from the orbit 
3155 posteroventral margin (158.0), mostly horizontal pterygoid with a distinct posterolateral angle

3156 (198.1) and cultriform process of the basisphenoid exposed and bifurcates the pterygoids (225.1).

3158 Registration number. To be added in proof.

3159 Phylogenetic definition. The largest clade within Teleosauroidea containing Machimosaurus 3160 hugii, but not Plagiophthalmosuchus gracilirostris and Teleosaurus cadomensis. This is a 3161 maximum-clade, or stem-based, definition.

3162 Reference phylogeny. Phylogenetic analyses presented herein, see figures 59-61 and 63-64.

3163 Composition. Charitomenosuchus, Clovesuurdameredeor, Deslongchampsina, Macrospondylus, 3164 Seldsienean and Machimosaurinae (comprising Andrianavoay, Neosteneosaurus, 3165 Proexochokefalos and Machimosaurini [comprising Lemmysuchus, Machimosaurus and 3166 Yvridiosuchus]).

3167 Diagnostic apomorphies. 34.1; 48.0; 58.1; 103.0; 158.0; 198.1; 225.1. (From the dataset herein, 3168 the same characters as the ICZN Code description.) Neosteneosaurus + Machimosaurini)

3171 Classification note. Machimosaurinae is a 'family group' clade established under the ICZN

3172 Code, at the subfamily rank. urn:Isid:zoobank.org:act:918AC1F1-AC04-41D1-91DA-

3173 E2D25939EAB9. 
3174 Nominal authority. The nominal authority is based on Article 36.1 of the ICZN Code (Principal 3175 of Coordination, applied to family group names).

3176 Description. The subfamily Machimosaurinae is supported by a handful of characters including 3177 the supratemporal fenestra length being twice as long as the width (104.1), a shallow Meckelian 3178 groove (269.1), a sharply curved angular (270.1) and non-procumbent dentition throughout the 3179 entirety of the jaws (325.0). Two of these characters are new to the dataset.

\section{Machimosaurinae nomen cladi novum}

3181 Registration number. To be added in proof.

3182 Phylogenetic definition. The largest clade within Teleosauroidea containing Machimosaurus

3183 hugii but not Deslongchampsina larteti and Charitomenosuchus leedsi. This is a maximum3184 clade, or stem-based, definition.

3185 Reference phylogeny. Phylogenetic analyses presented herein, see figures 59-61 and 63-64.

3186 Composition. Andrianavoay, Neosteneosaurus, Proexochokefalos and Machimosaurini 3187 (comprising Lemmysuchus, Machimosaurus and Yvridiosuchus).

3188 Diagnostic apomorphies. 104.1; 269.1; 270.1; 325.0. (From the dataset herein, the same 3189 characters as the ICZN Code description.)

3191 Synapomorphies. 66.0 . 
3192 Comments. The sole character supporting Proexochokefalos heberti and Proexochokefalos cf.

3193 bouchardi as sister taxa is the lack of a midline cavity (= trench) on the nasals, instead being flat 3194 (66.0).

\section{Machimosaurini Jouve et al., 2016 (Yvridiosuchus + Lemmysuchus + Machimosaurus)}

3196 Classification note. Machimosaurini is a 'family group' clade established under the ICZN Code, 3197 at the tribe rank.

3198 Description. A number of character states support the monophyly of Machimosaurini. These 3199 include parallelogram-shaped supratemporal fenestrae (102.5), blunt apices (327.1), no curvature 3200 in the middle to posterior dentition (345.0), false ziphodont serrations restricted to posteriorly3201 placed tooth crowns (349.2), incipient ziphodont carinae (351.2), rounded true denticles (352.1), 3202 all teeth microziphodont (353.1), strongly developed anastomosed pattern on the apices (358.1), 3203 three sacral vertebrae (379.1), sub-square ischial plate (449.1), ventrally angled tibial tuberosity 3204 (464.1), and keeled osteoderms with variable and elongated pits (473.3). Two of these characters 3205 are new to the dataset. Jouve et al. (2016) initially described the tribe Machimosaurini based on 3206 the following characteristic features: (1) shortened rostra; (2) enlarged supratemporal fenestrae; 3207 (3) reduced tooth counts; and (4) blunt, ornamented dentition.

3208 Comments. Certain characteristics of machimosaurins, particularly their teeth, have been 3209 documented for many years; Mac. hugii was first described by von Meyer in 1837, who made a 3210 particular comment about the dentition: “...stumpfkonischen und dicht gestreiften Zähnen

3211 besonders charakteristisch herauszustellen...” (“...particularly [conspicuous in] conical and 3212 densely striped teeth...") (von Meyer, 1837: 560). Sauvage and Liénard (1879: 7) noted "La 
3213 forme des vertèbres, la disposition des écussons, la composition de la tête [...], la forme et

3214 l'ornamentation des dents..." ("The shape of the vertebrae, the arrangement of the osteoderms,

3215 the composition of the head [...], the shape and ornamentation of the teeth...") when describing

3216 Mac. mosae. Phillips (1871: 184-185) also defined the teeth of Yvridiosuchus (known then as

3217 Teleosaurus brevidens; see Johnson, Young \& Brusatte, 2019) as “....rather short [teeth]...a little

3218 curved, uniformly striated, the striae growing more prominent toward the point and finer toward

3219 the base... [a] slight trace of bicarination on these teeth, near the apex, which is usually blunt...";

3220 he appears to be referring to the anastomosing pattern. Andrews (1913: 132), made note of the

3221 third sacral vertebra in Lemmysuchus, saying “... a remarkable condition is found, there being

3222 apparently three sacrals... [seems to be] that the ribs of the first caudal have greatly enlarged and

3223 resemble sacral ribs..." However, Andrews (1913) thought this to be a unique feature in

3224 Lemmysuchus, not taking into context the same condition seen in species of Machimosaurus.

3225 Recent papers have also highlighted several of these features, including: detailed

3226 descriptions of the dentition (Young \& Steel, 2014; Young et al., 2015a; Jouve et al., 2016);

3227 specific features of the skull (Hua, 1996; Young et al., 2014; Fanti et al., 2016; Johnson et al., 3228 2017; Johnson, Young \& Brusatte, 2019); reduction in the pelvic bones (Johnson et al., 2017);

3229 and the unique sacral anatomy (Martin \& Vincent, 2013; Young et al., 2014; Johnson et al., 3230 2017).

3232 Registration number. To be added in proof. 
3233 Phylogenetic definition. The largest clade within Teleosauroidea containing Machimosaurus

3234 hugii, but not Neosteneosaurus edwardsi. This is a maximum-clade, or stem-based, definition.

3235 Reference phylogeny. Phylogenetic analyses presented herein, see figures 59-61 and 63-64.

3236 Composition. Lemmysuchus, Machimosaurus and Yvridiosuchus.

3237 Diagnostic apomorphies. $102.5 ; 327.1 ; 345.0 ; 349.2 ; 351.2 ; 352.1 ; 353.1 ; 358.1 ; 379.1 ; 449.1$;

$3238464.1 ; 473.3$. (From the dataset herein, the same characters as the ICZN Code description.)

3239

Features uniting the genus Machimosaurus

3240 Unambiguous Synapomorphies. 7.0.

3241 Ambiguous Synapomorphies. 32.0; 288.3; 292.-; 293.-; 294.-; 297.-; 300.-; 395.\{01\}; 406.1.

3242 Comments. There are multiple features unique to the genus Machimosaurus; however, there is

3243 only one definitive character that is preserved in all species: a wider than higher rostrum (7.0).

3244 All ambiguous synapomorphies are found in both Mac. buffetauti and Mac. mosae, but are

3245 scored as (?) in Mac. hugii and Mac. rex due to lacking or fragmentary material. These

3246 synapomorphies include simple, straight-lined dentary neurovascular foramina (32.0), three

3247 premaxillary alveoli (288.3), the tuberculum and articular facet of dorsal ribs positioned halfway

3248 in the middle (395.\{01\}), scapula with a strongly concave anterior edge (406.1), and

3249 inapplicability of ch. 292 to 294,297 and 300.

3250

3251 Discussion 


\subsection{Areas of uncertainty}

3253 The above analyses, similar to recent studies (e.g. Ösi et al., 2018; Foffa et al., 2019; Johnson,

3254 Young \& Brusatte, 2019; Sachs et al., 2019a), find many aspects of the phylogeny to be

3255 consistent, including:

3256 1. Plagiophthalmosuchus gracilirostris as the basal-most teleosauroid;

3257 2. The recovery of two well defined families (Teleosauridae and Machimosauridae); and

3258 3. The tribe Machimosaurini is situated within Machimosauridae.

3259

Using our updated dataset, we consistently recover the subfamilies Teleosaurinae and

3260 Aeolodontinae, regardless of changes and/or additions to the dataset. However, positions of

3261 certain taxa regularly change. For example, $P r$. cf. bouchardi is recovered as unresolved with

3262 other members of Machimosaurinae in the strict consensus topology; however, in the extended

3263 implied weighting topologies it is recovered as the sister taxon to Pr. heberti, and in the equal

3264 rates Bayesian test, it is found separate from Pr. heberti and most closely related to

3265 Andrianavoay, Neosteneosaurus, S. rostromajor and Machimosaurini. With these degrees of

3266 uncertainty, the addition of new characters and teleosauroid taxa has only caused greater

3267 ambiguity in certain areas of the tree (especially in the unweighted consensus analysis). While it

3268 is undoubtedly important to carefully study, re-analyse and re-describe specimens, and discover

3269 new character data, the addition of new characters may not be the key in resolving these issues.

3270 More importantly, one of the major problems is that a single specimen, usually skull

3271 material, represents many of these species, such as the Chinese teleosauroid (IVPP V 10098), Pr.

3272 heberti, Clovesuurdameredeor and Andrianavoay. In some cases, these specimens are well 
3273 preserved and offer vital information (e.g. Pr. heberti), but there are certain ones that may be key

3274 intermediate forms but are too fragmentary to offer any substantial data (e.g. Andrianavoay).

3275 One contributing factor is that very little fossil prospection is taking place in localities where

3276 many of these specimens have been found (e.g. Toarcian outcrops in China, Bathonian locations

3277 in Madagascar, Upper Jurassic sites in Thailand). In addition, there are vast areas, particularly

3278 along the Gondwanan coasts of Africa and India, which have yielded promising material but

3279 have yet to be prospected properly (Phansalkar, Sudha \& Khadkikar, 1994; Dridi \& Johnson,

3280 2019). This represents a unique opportunity for future work, and the discovery of additional

3281 material for existing species will offer a greater resolution into teleosauroid evolution during the

3282 Middle to Upper Jurassic and into the Lower Cretaceous.

32831.2 Excluded taxa

3284 Certain taxa were omitted from our analysis because 1) the holotype was either destroyed or 3285 could not be located or 2) said taxa did not possess any other current substantial material. For 3286 example, Machimosaurus nowackianus, a specimen comprising of the anterior dentary from

3287 Ethiopia, was reported being housed in the GPIT in Tübingen (Young et al., 2014). After its

3288 initial description, many researchers attempted to locate it within the collection and were unable

3289 (recently, it has been reported as returned from loan in March 2017: R. Irmis, pers. comm.).

3290 There is one available photograph of the specimen (Young et al., 2014, from Huene 1938 fig. 1-

3291 4); however, it was shown only in a slightly blurred dorsal view, but more importantly, due to the

3292 sheer incompleteness of the specimen and lack of characteristic features, we omitted this taxon

3293 from our dataset. 
3295 dataset because the holotype (comprising of skull and mandibular material) was destroyed in 32961944 (Vignaud, 1995), and there was no other definitive existing material for this particular

3297 taxon; currently, line drawings are the only source of information available (see Saville, 1876;

3298 Lennier, 1887). While these are invaluable for research, we were wary to score an entire taxon

3299 using only drawings; there are many instances (especially during the $19^{\text {th }}$ and early $20^{\text {th }}$

3300 centuries) where figures were either altered, drawn to include missing skeletal elements, or

3301 interpreted as similar to other taxa (e.g. Andrews, 1913). The holotype of Teleosaurus geoffroyi

3302 Eudes-Deslongchamps, $1868 \mathrm{c}$ was based on three mandibular fragments, which J.A. Eudes-

3303 Deslongchamps considered distinct due to "...un nombre sensiblement inférieur de dents" (“...a 3304 significantly lower number of teeth") than T. cadomensis (Vignaud, 1995: 181). However, this 3305 specimen (now considered an objective junior synonym of T. cadomensis: see Jouve, 2009) was 3306 also destroyed in 1944, and this distinguishing feature cannot be confirmed. In addition, two taxa 3307 were disregarded due to specimens simply being too fragmentary. First, the holotype of 3308 Steneosaurus rudis Sauvage, 1874 consisted of fragmentary pieces of the skull and mandible; it 3309 was part of the BHN2R collection, which was later closed in 2003, and it went missing.

3310 However, Vignaud (1995) suggested that, due to the robustness of the specimen, it could be 3311 referred to as Machimosaurus sp. The second example is Steneosaurus roissyi Eudes-

3312 Deslongchamps, 1869 (MNHN.RJN 130a-c), which consists of a fragmentary piece of the 3313 mandible; this material has no distinguishing characteristics and is therefore more apt to be 3314 referred to as Teleosauroidea indeterminate.

3316 analyses. The first is Steneosaurus pictaviensis (Fig. 62A). Vignaud (1998: 30-31) described the 
3317 holotype (LPP.M.35; although this specimen is labelled as LPP.M.37 in collections) and

3318 paratype (LPP.M.37, although this is labelled as LPP.M.35 in collections) as being different from

3319 Steneosaurus (= Charitomenosuchus) leedsi in that:

3320 1. No antorbital fenestrae (only an underlying depression) were present in S. pictaviensis;

33212 2. The maxillae were "plus élevés" ("higher than") C. leedsi; and

3322 3. The interalveolar surface of the dentary was smooth and "sans les deux sillons

3323 longitudinaux" ("without the two longitudinal furrows"), unlike C. leedsi.

$3324 \quad$ However, these characters are erroneous; firstly, in C. leedsi (NHMUK PV R 3320;

3325 NHMUK PV R 3806; BRLSI GP1770a-e), the antorbital fenestrae are very small, shallow and 3326 depression-like. In LPP.M.37, there is a small depression where the antorbital fenestrae should 3327 be located, similar to C. leedsi. Secondly, the crania of many C. leedsi specimens (e.g. NHMUK 3328 PV R 3320; NHMUK PV R 3806; PETMG R179) are dorsoventrally crushed, so the maxillae 3329 appear to be low; however, BRLSI GP1770a-e is three-dimensionally preserved, with the 3330 maxillae dorsoventrally high as in LPP.M.37. Lastly, it is unclear what longitudinal furrows 3331 Vignaud (1998) was referring to in C. leedsi; the interalveolar surface of the dentary (NHMUKL 3332 PV R 3320; NHMUK PV R 3806) is smooth, with anteriorly prominent lateral crenulations 3333 similar to LPP.M.35. If Vignaud (1998) was referring to the coronoid processes protruding into 3334 the dentary, these are quite large in both LPP.M.35 and C. leedsi (NHMUK PV R 3320). In 3335 addition, LPP.M.35 and LPP.M.37 are comparable to C. leedsi (NHMUK PV R 3320; NHMUK 3336 PV R 3806) in the following:

3337 1. Frontal with few, circular pits that are largely concentrated in the centre of the bone;

3338 2. Mediolaterally thin posterior processes of the nasals (similar to T. cadomensis); 
3339

3340

3341

3342

3343

3344

3345

3346

3347

3348

3349

3350

3351

3352

3353

3354

3355

3356

3357 3358

3. Sub-rectangular supratemporal fenestrae;

4. Slender teeth with pointed apices and faint enamel ornamentation; and

5. All referred specimens are middle Callovian in age and are found in corresponding stratigraphic horizons.

Therefore, we consider $S$. pictaviensis as a subjective junior synonym of C. leedsi.

The second taxon is Steneosaurus depressus Phizackerley, 1951 (OUMNH J.01420) (Fig. 62B). Phizackerley (1951) defined this a distinct species based on the following features: (1) the delicately constructed skull; (2) a slender, rounded rostrum comprising $64 \%$ of the total skull length; (3) small orbits; (4) small, slender, curved teeth; and (5) mandibular symphysis occupying roughly $48 \%$ of the entire mandible. However, these features can be attributed to subadult specimens or are found in other teleosauroid taxa. In addition, OUMNH J.01420 shares the following combination of key characteristics seen in Pr. heberti (MNHN.F 1890-13):

1. Enlarged occipital tuberosities (differs from all other members of Teleosauroidea);

2. No antorbital fenestrae;

3. Elongated, slender anterior process of the jugal; and

4. The $\mathrm{P} 1$ is oriented anteriorly and the $\mathrm{P} 2$ is oriented slightly medially (differs from Neosteneosaurus NHMUK PV R 3701).

Therefore, $S$. depressus can tentatively be referred to as a subjective junior synonym of Pr. heberti. However, a thorough re-description of both specimens is needed and is beyond the scope of this paper. 
3360 from our dataset as its holotype likely represents a sub-adult individual. The vertebral

3361 neurocentral suture is visibly prominent in young modern crocodylians and gradually closes and

3362 disappears in adults, in the direction from the caudals to the cervicals (Brochu, 1996). In the $S$.

3363 hulkei holotype, the neurocentral sutures are clearly visible and well-developed in the posterior

3364 thoracic vertebrae, suggesting it was a juvenile or sub-adult. In addition, S. hulkei displays a

3365 mixture of features similar to those seen in Neosteneosaurus (NHMUK PV R 2865; PETMG

3366 R178) and differs from Charitomenosuchus (NHMUK PV R 3320, NHMUK PV R 3806) and

3367 Lemmysuchus (NHMUK PV R 3168), such as:

3368 1. The cranium is overall more robust than Charitomenosuchus (NHMUK PV R 3320);

3369 2. No antorbital fenestrae are present (differs from Charitomenosuchus [NHMUK PV R 3320,

$3370 \quad$ NHMUK PV R 3168] in which they are present);

3371 3. A subcircular premaxilla-maxilla suture (differs from Charitomenosuchus [NHMUK PV R

3372 3320], which has a strongly interdigitating, rectangular premaxilla-maxilla suture);

3373 4. Dorsoventrally short supraoccipital (differs from Lemmysuchus [NHMUK PV R 3168] in

3374 which the supraoccipital is dorsoventrally tall);

3375 5. Deep reception pits until the posterior region of the maxilla (differs from Charitomenosuchus

3376 [NHMUK PV R 3806] which has deep reception pits until the mid-maxilla, and

3377 Lemmysuchus [NHMUK PV R 3168] which has deep reception pits along the entirety of the

3378 maxilla);

3379 6. Straightened posteriorly placed cervical ribs (differs from Lemmysuchus [NHMUK PV R

$33803168]$ which has a curved posteriorly placed cervical rib); 
3381 7. Triangular-shaped ischial blade and elongated anterior iliac process (differs from

3382 Lemmysuchus [NHMUK PV R 3168] in which the ischial blade is sub-square and the anterior

3383 iliac process is shortened); and

3384 8. Two sacral vertebrae (differs from Lemmysuchus [NHMUK PV R 3168] which has three 3385 sacrals).

3386

Therefore, $S$. hulkei can tentatively be referred to as a juvenile individual of

3387 Neosteneosaurus.

3388

\subsection{Ecomorphological diversity}

3389 Our new phylogeny clarifies key ecomorphological aspects of teleosauroids, some of which have 3390 briefly been discussed in the literature. The ecological structuring of teleosauroids was initially 3391 outlined by Hua (1997) and Hua \& Buffetaut (1997) but was never discussed or published in 3392 detail. Massare (1987) and recently Foffa et al. (2018a) characterized a variety of fossil marine 3393 reptiles based on features of the teeth, separating various taxa into dietary guilds. In Foffa et al. 3394 (2018a), seven teleosauroid taxa were included in the analysis. The results showed that 3395 Machimosaurus and Lemmysuchus occupied the crunch guild, which is specialized for handling 3396 hard prey (e.g. turtles); the remaining taxa (Mycterosuchus, Charitomenosuchus, 3397 Neosteneosaurus and Proexochokefalos) fit into the pierce guild, hypothesized to prefer softer 3398 prey such as smaller fishes and squid.

3400 exhibit a distinct pattern of appearance, and there are four well-sampled points during the

3401 Jurassic (Toarcian, Bathonian, Callovian and Kimmeridgian) in which specific patterns of 
3402 ecomorphotypes emerge (see Table 1; Fig. 63). These ecomorphs can be generally defined based

3403 on skull shape (longirostrine, mesorostrine or brevirostrine), dentition (for possible feeding style)

3404 and additional osteological characters that relate to the environment (e.g. length of the limbs,

3405 placement of the orbits). Teleosauroid skulls are generally split into three different 'rostral

3406 morphs': longirostrine, mesorostrine and brevirostrine (Fig. 63A), which relate to the length of

3407 the rostrum. Longirostry (e.g. Mycterosuchus) is defined as the preorbital length being $70 \%$ or

3408 more of the basicranial length; mesorostry (e.g. Mystriosaurus) is the preorbital length being 55-

$340970 \%$ of the basicranial length; and brevirostry (e.g. Mac. mosae) is the preorbital length being

$341055 \%$ or less than the basicranial length (Andrade et al., 2011). This rostral classification is in turn

3411 affiliated with features of the teeth, which include overall size and shape of the teeth, shape of

3412 apices, and presence or absence of carinae and ornamentation. In addition to these 'rostral

3413 morphs', teleosauroid feeding ecology can be broadly categorized into two feeding 'guilds':

3414 specialist (a species that has a limited diet) or generalist (a species able to thrive on a wide

3415 variety of food sources), which can be inferred based on the shape, size and apices of their teeth

3416 (Feranec, 2007). Macrophagous/durophagous (feeding on hard prey items) is generally regarded

3417 as part of the generalist guild (Foffa et al., 2018), but for the purpose of this paper, we refer to it 3418 separately.

3419 During the Toarcian, Plagiophthalmosuchus represented a longirostrine specialist (Fig.

3420 63A-B), characterized by its laterally facing orbits, elongated snout and multiple thin, pointed,

3421 poorly ornamented teeth, and was likely purely piscivorous (Westphal, 1962). Macrospondylus

3422 represents a longirostrine generalist and Mystriosaurus is a mesorostrine generalist (a massive,

3423 less elongated skull with smaller supratemporal fenestrae and more robust teeth). A heavily

3424 armoured, semi-terrestrial longirostrine generalist form is found in Platysuchus, indicated by the 
3425 extensive and tightly packed rows of dorsal osteoderms. It is difficult to discern which

3426 ecomorphotype the Chinese teleosauroid (IVPP V 10098) fits into, as no teeth are preserved.

3427 However, based on both anatomical and phylogenetic data, this taxon would hypothetically have

3428 filled a mesorostrine role, possibly a generalist, similar to Mystriosaurus (which is a logical 3429 assumption, given Mystriosaurus is a closely related taxon).

3430 By the Bathonian, basal teleosauroids with laterally oriented orbits had presumably

3431 become extinct (only being known from the Toarcian), with the Plagiophthalmosuchus

3432 ecomorph vacated (and possibly held by basal metriorhynchoids). However, a new

3433 ecomorphotype had evolved: the macrophagous/durophagous mesorostrine form, exhibited by

3434 Yvridiosuchus. A number of specific features, including enlarged supratemporal fenestrae, an

3435 extensive neurovascular system and blunt, conical teeth, characterized this ecomorphotype. The

3436 larger supratemporal fenestrae would have housed powerful adductor muscles for closing the

3437 jaw, and the robust, rounded teeth were advantageous for capturing a wider or more generalised

3438 range of prey (Johnson et al., 2017). There has also been some speculation that the evolution of

3439 machimosaurin features may have been linked to the evolution of hard shells in turtles; however,

3440 this possible correlation is difficult to test, due to the overall extreme diversification and

3441 expansion of coastal marine ecosystems (M. Rabi, pers. comm.). In addition to the

3442 durophagous/macrophagous role, Seldsienean filled the longirostrine generalist niche;

3443 Deslongchampsina filled the niche of mesorostrine generalist; and Teleosaurus replaced

3444 Platysuchus as the longirostrine, semi-terrestrial generalist form. The possible ecomorphotypes

3445 for both Andrianavoay and Clovesuurdameredeor are currently uncertain; morphologically it is

3446 clear that they do not represent machimosaurins (e.g. lack two rows of maxillary neurovascular

3447 foramina in Andrianavoay; no enlarged supratemporal fenestrae in Clovesuurdameredeor). Most 
3448 of the rostral material is missing from Clovesuurdameredeor, making it difficult to infer skull

3449 and dental morphology. The preserved rostral section (including the anterior and middle

3450 maxillae) of Andrianavoay has at least 20 maxillary alveoli preserved; due to its position on the

3451 phylogeny, it may possibly have been a mesorostrine generalist, similar to Neosteneosaurus.

3452 In the mid-Callovian, the ecomorphotypes within this ecological hierarchy did not

3453 change. Lemmysuchus represented a mesorostrine macrophagous/durophagous form;

3454 Charitomenosuchus became the longirostrine generalist; Neosteneosaurus and Pr. heberti both

3455 filled the role of mesorostrine generalist; and Mycterosuchus represented the longirostrine, semi-

3456 terrestrial ecomorphotype. However, in the Kimmeridgian, there was another major shift in

3457 ecomorphotype variation. The macrophagous/durophagous form became the most dominant

3458 ecomorph, with representatives in Mac. buffetauti, Mac. mosae (both brevirostrine) and Mac.

3459 hugii (mesorostrine). The semi-aquatic longirostrine generalist ecomorph disappeared, and the

3460 mesorostrine generalist, represented by $P r$. cf. bouchardi, became extremely rare. In addition,

3461 another new ecomorphotype evolved: a longirostrine, semi-pelagic generalist form, represented

3462 by a handful of genera (Aeolodon, Bathysuchus and Sericodon). During the Upper Jurassic (the

3463 exact time is unknown), Indosinosuchus represented a probable generalist, mesorostrine form;

3464 and in the Hauterivian-Barremian (132 to $121 \mathrm{Ma}$ ), Mac. rex embodied the

3465 macrophagous/durophagous ecomorph, but all other teleosauroids had presumably disappeared.

3466 These six different ecomorphotypes are scattered across the phylogeny.

3467 Plagiophthalmosuchus, the basal-most teleosauroid, is the only taxon that is a definitive

3468 longirostrine specialist (Fig. 63). Mesorostrine generalists are represented by both teleosaurids

3469 and machimosaurids: the Chinese teleosauroid (IVPP V 10098), Mystriosaurus and 
3470 Indosinosuchus (Teleosauridae); and Deslongchampsina, Proexochokefalos, and

3471 Neosteneosaurus (Machimosauridae) (Fig. 63). Interestingly, the remaining three

3472 ecomorphotypes are restricted to certain families. The longirostrine semi-terrestrial form is only

3473 found in Teleosauridae, represented by Platysuchus, Teleosaurus and Mycterosuchus. The

3474 longirostrine, generalist pelagic ecomorphotype is also restricted to Teleosauridae, as seen in

3475 Aeolodon, Sericodon and Bathysuchus (Fig. 63A-C). The longirostrine generalist

3476 (Macrospondylus, Seldsienean, Charitomenosuchus) and mesorostrine/brevirostrine

3477 macrophagous/durophagous (Yvridiosuchus, Lemmysuchus, Machimosaurus) ecomorphologies

3478 are only found in Machimosauridae (Fig. 63).

3479

As seen in extant crocodylian species, larger individuals tend to be dominant, with larger

3480 species occupying prime territories, although this is not an unbreakable rule, as interactions

3481 between Crocodylus rhombifer (Cuban Crocodile) and Crocodylus acutus (American Crocodile)

3482 in the Central Americas demonstrate (Targarona et al., 2010; Thorbjarnarson, 2010). It is

3483 hypothetical that machimosaurids, being larger and more generalist, were able to assert

3484 dominance over smaller teleosaurids if co-existing within the same ecosystem, and therefore

3485 occupied more prime territories. This could have acted as a selection pressure and driven the

3486 evolution of more specialised ecomorphotypes. This is similar to that seen in extant crocodylian

3487 subdivisions of West African ecosystems; the species Crocodylus suchus (West African

3488 Crocodile), Mecistops cataphractus (West African slender-snouted crocodile) and Osteolaemus

3489 tetraspis (African Dwarf Crocodile) do not inhabit similar bodies of water (e.g. Kofron, 1992;

3490 Velo-Antón et al., 2014), and with decreasing size, all species live in smaller waterways, with

3491 Osteolaemus being capable of terrestrial foraging. This could be similar to the hierarchy seen in

3492 South American caimans: Melanosuchus niger (Black Caiman), Paleosuchus palpebrosus 
3493 (Cuvier's Dwarf Caiman), Caiman yacare (Yacare Caiman), Caiman crocodilus (Spectacled

3494 Caiman) and Caiman latirostris (Broad-Snouted Caiman) (Ross, 1998; Busack \& Pandya, 2001;

3495 Rebêlo \& Lugli, 2001; Vasconcelos et al., 2006).

3496

An additional interesting factor is that, throughout time, there were never more than four

3497 ecomorphological 'guilds' within teleosauroids (Fig. 64). Mesorostrine generalists (e.g.

3498 Deslongchampsina) and longirostrine generalists (e.g. Charitomenosuchus) were consistently

3499 present until the Late Jurassic, whereas the basal longirostrine specialist (Plagiophthalmosuchus)

3500 was present only during the Early Jurassic. During the Kimmeridgian/Tithonian, there were only

3501 three ecomorphs present (Fig. 64) (macrophagous/durophagous, longirostrine pelagic, and

3502 mesorostrine generalist forms) with two of these (macrophagous/durophagous and longirostrine

3503 pelagic forms) being dominant while the third (mesorostrine generalist form) was much rarer. In

3504 addition, Young et al. (2014) noted that, during the Late Jurassic, there was a divide within the

3505 genus Machimosaurus between 'open-sea’ Machimosaurus body-plans (i.e. Mac. hugii, as

3506 suggested by the enlarged paraoccipital processes for muscle attachment) and

3507 nearshore/turbulent water body-plans (i.e. Mac. mosae). The overall reflection of teleosauroid

3508 nice partitioning highlights three main points:

3509 1. There was a specific niche partitioning strategy among teleosauroids that lived during

$3510 \quad$ similar times;

35112 . The ecomorphological diversity of teleosauroids was generally stable through time until

3512 the Late Jurassic; and

3513 3. After the Late Jurassic, there was a growing divide within Teleosauroidea between near-

3514 shore forms and increasingly open-sea species. 


\section{1.4 Biogeographical distribution}

3516 Throughout their approximately 70-million-year history, teleosauroids achieved near-global

3517 distribution. Numerous specimens have been found across both Gondwanan and Laurasian

3518 continents, having been reported from the UK and Europe (Eudes-Deslongchamps, 1867-69;

3519 Westphal, 1961, 1962; Andrews, 1909, 1913; Benton \& Taylor, 1994; Young et al., 2014;

3520 Johnson et al., 2017; Čerňanský et al., 2017; Foffa et al., 2019), Africa (Newton, 1893; De

3521 Lapparent, 1955; Buffetaut, Termier \& Termier, 1981; Bardet \& Hua, 1996; Fara et al., 2002;

3522 Fanti et al., 2016; Jouve et al., 2016; Dridi \& Johnson, 2019), Asia (Young, 1948; Liu, 1961; Li, 3523 1993; Martin et al., 2019), India (Owen, 1852; Phansalkar, Sudha \& Khadkikar, 1994), Siberia

3524 (Efimov 1982, 1988; Storrs \& Efimov, 2000), South America (Cortés et al., 2019) and

3525 potentially North America (Table 2). Von Huene (1927) described two dorsal vertebrae from the

3526 Upper Lias of Portezuelo Ancho in north-western Argentina and attributed them to Steneosaurus

3527 gerthi (Buffetaut, 1981; Gasparini \& Fernández, 2005); however, these specimens are now 3528 referred to as Thalattosuchia indeterminate (Gasparini \& Fernández, 2005).

3529

Despite this vast global dispersal, few studies have examined teleosauroid biogeography

3530 in detail. Buffetaut et al. (1981) suggested a Laurasian and Gondwanan faunal connection

3531 between Tethyan Europe and the southern area of Africa (such as Madagascar) via an 3532 epicontinental seaway during the Early Jurassic. In the late Toarcian, the distribution of 3533 teleosauroids appear parallel to the ammonite Bouleiceras, which occurs in Portugal (Mouterde,

3534 1953), Spain (Geyer, 1956), Chile, Argentina (von Hilldebrandt, 1973), Madagascar, Algeria and

3535 Morocco (Buffetaut, Termier \& Termier, 1981), suggesting a marine connection from South

3536 America around Africa to the Tethyan area. In addition, Hua \& Buffetaut (1997) hypothesized 
3537 that teleosauroid distribution was similar to that of the Saltwater Crocodile (Crocodylus porosus)

3538 living amongst the Indian Ocean archipelagos.

3539 Fossil localities appear to reflect the biogeographical diversity of teleosauroids. During

3540 the upper Toarcian, teleosauroids were already biogeographically distinct. Representatives from

3541 both Teleosauridae and Machimosauridae, as well as the basal teleosauroid

3542 Plagiophthalmosuchus, are found in the Whitby Mudstone Formation in Britain (Mystriosaurus,

3543 Macrospondylus), the 'schistes bitumineux' in Luxembourg (Macrospondylus, Platysuchus), an

3544 unknown locality in France (Macrospondylus) and the Posidonia Shale Formation in Germany

3545 (Platysuchus, Macrospondylus, Mystriosaurus). In Asia, the Chinese teleosauroid and

3546 indeterminate 'Teleosaurus' material are noted from the Ziliujing Formation of Beipei, Sichuan

3547 in China (Li, 1993; Li et al., 2011). In addition, Toarcian Steneosaurus specimens have been

3548 reported from Belgium ('oolithe ferrugineuse'), India (Kota Formation), Madagascar (Kandreho

3549 Formation), and possibly Portugal (Owen, 1852; Buffetauti et al., 1981; Godefroit, 1994). These

3550 multiple occurrences in different localities indicate that during the beginning of teleosauroid

3551 evolution, they were already radiating across the world, possibly following the coastline.

During the Aalenian and Bajocian (180.1 to 169.2 Ma), there are few teleosauroid

3553 occurrences, but there are two geographically important 'Steneosaurus' sp. found in Slovakia

3554 (Pieniny Klippen Belt unit; Aalenian) and Dagestan Republic (Karakh Formation; Aalenian).

3555 During the Middle Jurassic (Late Aalenian to Early Bajocian), Buffetaut (1979) reported

3556 teleosauroid material from Oregon (USA); this material has since been attributed to a member of

3557 Metriorhynchoidea (Wilberg, 2015b). However, some non-documented, additional fragments

3558 from the same timeframe and locality are still labelled as Teleosauridae (NMNH PAL 357211 to 
3559 357215). In the Bathonian (169.2 to $164.4 \mathrm{Ma}$ ), several teleosauroid genera have been reported

3560 from localities in France (Yvridiosuchus, Teleosaurus, Seldsienean, Deslongchampsina,

3561 'Steneosaurus'; Eudes-Deslongchamps, 1867-68; Johnson, Young \& Brusatte, 2019), Britain

3562 (Clovesuurdameredeor, Yvridiosuchus, Teleosaurus, Seldsienean, Deslongchampsina; Eudes-

3563 Deslongchamps, 1867-68; Johnson, Young \& Brusatte, 2019), Madagascar (Andrianavoay;

3564 Newton, 1893) and Morocco (Machimosaurini indeterminate).

3565

There is a multitude of occurrences in the Callovian (164.4 to $159.4 \mathrm{Ma}$ ), particularly in

3566 Britain (Oxford Clay Formation): taxa found in this area include Mycterosuchus,

3567 Charitomenosuchus, Neosteneosaurus and Lemmysuchus. Teleosauroids such as

3568 Proexochokefalos (Marnes de Dives Formation), Lemmysuchus (Quercy) and 'Steneosaurus' sp.

3569 (unknown formation) are found in France, as well as 'Steneosaurus' sp. (Chari Formation) in

3570 India. As with the Aalenian-Bajocian, few teleosauroids have been reported from the Oxfordian

3571 (159.4 to $154.1 \mathrm{Ma}$ ). However, there are a couple of specimens described from unique localities, 3572 such as:

3573

3574

3575

3576

3577

3578

3579

3580
1. Machimosaurus nowackianus from Harrar, Ethiopia (von Huene, 1938; Bardet \& Hua; Young et al., 2014);

2. Machimosaurus sp. (Perisphinctes cautisnigrae ammonite zone) and L. cf. obtusidens (Corallian Group; Foffa, Young \& Brusatte, 2015) from Britain; and

3. Steneosaurus rostromajor (possibly Marnes de Villiers Formation; Cuvier, 1812, 1824; Geoffroy Saint-Hilaire, 1825) from France.

In the Kimmeridgian (154.1 to $150.7 \mathrm{Ma}$ ), teleosauroids are found in several localities: Bathysuchus from the Kimmeridge Clay Formation (UK); Mac. hugii, Sericodon and Pr. cf. 
3581 bouchardi from the Reuchenette Formation (Switzerland); Mac. buffetauti from the

3582 Lacunosamergel Formation (Germany); Mac. hugii from the Alcobaça and Lourinhã Formaions

3583 (Portugal), as well as the Lastres and Tereñes Formations (Spain) and Calcaires Coquilliers

3584 Formation (P. baylei Sub-Boreal ammonite Zone; Cricqueboeuf, France); and Pr. cf. bouchardi

3585 from the 'Calcaire de Caen' (France) (e.g. Lepage et al., 2008; Young et al., 2014; Schafer et al.,

3586 2018; Foffa et al., 2019). In addition, Machimosaurus sp. is found in Germany (Langenberg

3587 Formation), the UK (Kimmeridge Clay Formation), Switzerland (Reuchenette and unknown

3588 Formations) and Portugal (Lourinhã Formation) (e.g. Young \& Steel, 2014; Young et al., 2014),

3589 and 'Steneosaurus' sp. has been found from the Czarnogłowy quarry in Poland (Čerňanský et al.,

3590 2017). Tithonian localities are restricted to the Higueruelas Formation in Spain (Mac. hugii), the

3591 Mörnsheim Formation in Germany (Aeolodon) and the Canjuers lagerstätte and 'Marnes

3592 supérieures de la Meuse' in France (Aeolodon and Mac. mosae, respectively). Indosinosuchus

3593 comes from the Late Jurassic Phu Kradung Formation of Phu Noi (north-eastern Thailand);

3594 dating this stratigraphic section is particularly tricky, as vertebrate fossils indicate a Late Jurassic

3595 age but palynomorphs suggest Early Cretaceous (Martin et al., 2019). A Late Jurassic, possibly

3596 Tithonian, age has been proposed (e.g. Liard and Martin, 2011; Cuny et al., 2014; Deesri et al.,

3597 2014; Liard et al., 2015), but this is currently unconfirmed.

$3598 \quad$ Two geographically important specimens have been attributed to the genus

3599 'Steneosaurus': a partial skull from the Karakh Formation (Aalenian) of Dagestan, Russia

3600 (Efimov, 1988), and two skulls from the Chari Formation (Callovian) near Gujarat, India

3601 (Phansalkar, Sudha \& Khadkikar, 1994). The Dagestan skull (Efimov, 1988) was housed at the

3602 Grozny Petroleum Research Institute (GrozNII) in the Chechen Republic but was destroyed due

3603 to military conflict in the area (S. Zaurbekov, pers. comm.). This is unfortunate, not only in the 
3604 loss of three valuable specimens, but also in the fact that their unique locations would provide

3605 invaluable information on which teleosaurids and/or machimosaurids spread into these areas.

3606 Efimov (1988) described the Dagestan skull as "Вместе с тем в конфигурации краниальной

3607 пластины она обнаружсивает сходство с верхнеюрскими видами стенеозавра, в

3608 частности сS. larteti $u$ S. edwardsi" ("At the same time, in the configuration of the cranial

3609 plate, it reveals similarities with the Upper Jurassic species [of] Steneosaurus, in particular, S.

3610 larteti and S. edwardsi") (Efimov, 1998: 52). However, there are no photographs of the

3611 specimen, so this is difficult to confirm. Currently, the Gujarat skulls cannot be located; in

3612 addition, Phansalkar, Sudha \& Khadkikar (1994) did not describe either of the Gujarat

3613 specimens, only noting their occurrence within the Chari Formation. There is one photograph of

3614 one skull, as well as two drawings, but they are poor, and no anatomical information can be

3615 gleaned from them. Khadkikar (1996) briefly noted the skulls, suggesting that they could belong

3616 to $S$. durobrivensis (=S. edwardsi = Neosteneosaurus). Nevertheless, these specimens exhibit the

3617 remarkable distributional success and adaptability that teleosauroids were able to achieve.

3618 Based on the biogeography of the above fossil sites, it appears that teleosauroids

3619 primarily diversified and dispersed around the Tethys Sea (which was a productive area,

3620 consisting of many continental reef ecosystems: Stanley, 1988), and most species were

3621 concentrated around the Jurassic tropic belts. This is also consistent with climate data (Rees et

3622 al., 2000; Jenkyns et al., 2012; Korte et al., 2015), which suggests rapid warm/cool events

3623 influenced by oceanic currents followed by warm conditions ( 26 to $30^{\circ} \mathrm{C}$ ) during the Middle

3624 Jurassic, as well as overall minimal global climate change throughout the Jurassic, making the

3625 coastlines exceptionally productive. However, there are still three main problems which continue

3626 to limit our understanding of teleosauroid dispersal and distribution through time. Firstly, there is 
3627 a substantial area where material is either missing or severely fragmentary, including the Tethys

3628 coast of Africa and the eastern coast of Africa (ranging from Ethiopia to Madagascar). Secondly,

3629 the lack of confident identification for the lost Chechen material (Aalenian), and the Indian

3630 (Toarcian and Callovian) and Chinese (Toarcian) specimens limits our knowledge of which

3631 species of teleosauroids were able to successfully disperse into these areas. Lastly, the South

3632 American record for teleosauroids is surprisingly non-existent, as they are known only from the

3633 Early Cretaceous (Cortes et al., 2019). As teleosauroids must have dispersed through multiple

3634 routes along the Jurassic coastlines, it would be logical that they were able to migrate into the

3635 South American area during this time. It is therefore essential that future research examines

3636 material from, as well as exploring more of, these areas. As with patterns in teleosauroid

3637 ecomorphology, genera within both families were established in different locations (see Table 2).

3638 Teleosauridae were restricted to Laurasian continents, with Teleosaurus, Aeolodon,

3639 Mystriosaurus and Bathysuchus known from the UK and Europe; Mycterosuchus from Britain

3640 and Germany; Platysuchus from Europe (Germany and Luxembourg); and Indosinosuchus and

3641 the Chinese teleosauroid (and possibly Teleosaurus) from Asia. Machimosauridae have an

3642 overall wider geographical span, ranging from the UK and Europe to northern Africa,

3643 Madagascar and possibly India, with machimosaurins in particular being prevalent in Africa. The

3644 phylogeny also shows that teleosauroids were able to distribute across the continent early in their

3645 evolution; Plagiopthalmosuchus, three teleosaurids (Mystriosaurus, Platysuchus, the Chinese

3646 teleosauroid) and one machimosaurid (Macrospondylus) were definitively present during the

3647 early Toarcian in five distinct localities.

36481.5 Palaeoenvironment and the importance of freshwater teleosauroids 
3649 The majority of teleosauroid species are found in semi-aquatic or marginal marine (generally

3650 coastal and lagoonal) environments, and certain taxa are hypothesized to have lived in semi-

3651 pelagic (Aeolodon, Bathysuchus and Sericodon), semi-terrestrial (Mycterosuchus, Teleosaurus

3652 and Platysuchus) and open ocean (Mac. hugii) ecosystems (refer to Fig. 63C). However, three

3653 purely East Asian teleosauroids, the Chinese teleosauroid (IVPP V 10098) and two species of

3654 Indosinosuchus, are found in freshwater deposits (Li, 1993; Martin et al., 2016, 2019). This is

3655 intriguing, as no other teleosauroids are known from these types of deposits. In environmental

3656 terms, this is striking with reference to two points: (1) adult vs juvenile habitat preference; and

3657 (2) specific osteological features.

3658

Some modern crocodylians, such as Cr. porosus (Saltwater Crocodile), often prefer

3659 different habitats depending on their age (juvenile/sub-adult vs. adult) (Read et al., 2004), which

3660 is often related to body size and food preference (Taylor, 1979; Magnusson, da Silva \& Lima,

3661 1987). In general, adults are more common in estuary or brackish regions, whereas juveniles and

3662 sub-adults prefer freshwater ecosystems such as rivers or lakes. It is possible that teleosauroids

3663 adopted a similar pattern, with mature individuals frequenting semi-marine habitats, and

3664 hatchlings and juveniles in freshwater environments. However, small specimens of

3665 Macrospondylus (less than $1 \mathrm{~m}$ total length) have been found in the Posidonia Shale Formation

3666 from Holzmaden (e.g. SMNS 10 000), which consists of marginal marine sedimentological

3667 deposits. In addition, adult individuals of Cr. porosus (Webb, Manolis \& Brien, 2010),

3668 Crocodylus acutus (American Crocodile) (Thorbjarnarson et al., 2006) and possibly Crocodylus

3669 siamensis (Siamese Crocodile) (Smith, 1931; Platt et al., 2006)) have been known to thrive in

3670 both saltwater and freshwater ecosystems. 
3671 Certain osteological characteristics in mature individuals can also be indicative of

3672 preferential habitat. The Indian gharial (Gavialis gangeticus), which is confined to riverine

3673 ecosystems, has distinctive protruding eyes (= telescoped orbits) that aid in capturing fish

3674 (Whitaker \& Basu, 1983). In gavialoids, these telescoped orbits are homoplastic and

3675 independently evolved twice, once in advanced Gryposuchus species (Gr. colombianus and Gr.

3676 croizati) from South America, and once in Asian Gavialus (Salas-Gismondi et al., 2016). The

3677 depositional settings in which these taxa are found are fluvial-dominated paleoenvironments,

3678 which suggests that well-developed telescoped orbits are correlated with riverine ecosystems

3679 (Salas-Gismondi et al., 2016). In teleosauroids, Indosinosuchus potamosiamensis displays

3680 distinctive telescopic orbits (although not as widely separated as Gavialis) and is found in

3681 freshwater deposits (Martin et al., 2019), similar to Gryposuchus species. It would therefore be

3682 logical to assume that Indosinosuchus kalasinensis, from the same deposits, would also have had

3683 telescoped orbits; however, the skull (PRC-239) is slightly dorsoventrally crushed, making this

3684 confirmation difficult. Interestingly, Mycterosuchus nasutus, and more subtly Teleosaurus

3685 cadomensis, have telescoped orbits; it is thus hypothesized that these two taxa may have also

3686 preferred riverine/fluvial areas rather than marginal marine ecosystems.

In other fossil crocodylomorphs, the dyrosaurid Acherontisuchus guajiraensis Hastings,

3688 Bloch \& Jaramillo, 2011 is hypothesized to have inhabited calmer, fluvial waters than other Old

3689 World dyrosaurids. The slender and narrow ischial shaft of this taxon had reduced surface area

3690 for attachment surfaces of the $m$. rectus abdominis and $m$. ischiopubis, which are responsible for

3691 respiration and pitch control in water (Hastings, Bloch \& Jaramillo, 2011). The ischial shaft in

3692 teleosauroids is not as narrow or elongated as in dyrosaurids; the ischial shaft of the supposed

3693 fluvial I. potamosiamensis (PRC-27: Martin et al., 2019) does not look particularly different 
3694 from the majority of teleosauroids (e.g. Charitomenosuchus, Neosteneosaurus), excluding

3695 machimosaurins (e.g. Lemmysuchus). In addition, the sedimentology (Cerrejón Formation,

3696 Colombia) along with associated flora and fauna, suggest that A. guajiraensis lived in a

3697 freshwater habitat. All specimens of A. guajiraensis are mature individuals, with specimens

3698 ranging from 4.6 to $6.4 \mathrm{~m}$ in length (Hastings, Bloch \& Jaramillo, 2011). Adult specimens of the

3699 pholidosaurids Sarcosuchus, Elosuchus and Meridiosaurus are also thought to have inhabited

3700 freshwater ecosystems (Fortier, Perea \& Schultz, 2011). Therefore, it is possible that mature

3701 teleosauroids did indeed frequent freshwater ecosystems, but solely in eastern Laurasian regions.

3702 More discoveries are needed from freshwater deposits in Europe to test whether many marginal

3703 marine teleosauroids were solely marine taxa.

3704 One additional salient feature of teleosauroids is the position of the external nares. They

3705 are described as being either anterodorsally (e.g. in Indosinosuchus) or dorsally (e.g. in

3706 Deslongchampsina) oriented. However, in Mystriosaurus, the external nares are directed

3707 anteriorly (Sachs et al., 2019a). This is intriguing, as this positioning would not be practical for a

3708 semi-aquatic lifestyle. It is hypothetical that, due to this unusual placement of the external nares,

3709 Mystriosaurus was more terrestrial, or spent a greater amount of time on land, than other

3710 teleosauroids. Indeed, this example shows just how possible it is that some teleosauroids were, in

3711 actuality, not particularly well suited for living in water.

3712 1.6 Teleosaurids vs machimosaurids

3713 In terms of morphology and ecology, teleosaurids are more phenotypically plastic than

3714 machimosaurids (see Fig. 63). They display three distinct ecomorphs (mesorostrine generalist,

3715 longirostrine specialist and longirostrine generalist) and potentially occupied four environmental 
3716 habitats (semi-marine, pelagic, freshwater and semi-terrestrial). In contrast, machimosaurids

3717 seem to display an almost linear pattern: basal machimosaurids (e.g. Macrospondylus) are

3718 longirostrine, semi-marine generalists; more derived machimosaurines (e.g. Deslongchampsina,

3719 Proexochokefalos) are mesorostrine, semi-marine generalists, with more robust teeth; and

3720 machimosaurins (e.g. Lemmysuchus, Machimosaurus) are large-bodied, durophagous, semi-

3721 marine taxa, with complex dentition and robust skeletons. In terms of abundance and

3722 geographical dispersal, teleosaurids appear to be less common than machimosaurids, and based

3723 on current knowledge, were restricted to Laurasia. Machimosaurids as a whole, particularly

3724 Macrospondylus, have high abundance, and decrease in numbers after the Callovian. During the

3725 Kimmeridgian, Machimosaurus was the most common teleosauroid genus, but was less abundant

3726 than other contemporaneous marine reptiles. The distribution of machimosaurids is generally in

3727 Sub-Boreal European and Gondwanan areas and their dispersal was expansive, with multiple

3728 occurrences found in the UK, Europe and Africa, and potentially India. However, there is a

3729 possible instance of them being found in Siberia (see above). It is possible that machimosaurids

3730 had larger ranges than contemporaneous teleosaurids, with teleosaurids being more specialized

3731 and therefore restricted to certain environments. These ideas, reinforced by the phylogeny, show

3732 that teleosauroids were without doubt much more diverse, in terms of morphology, ecology and

3733 geography, than previously thought.

3734

An additional factor that differs between teleosaurids and machimosaurids is body size.

3735 Machimosaurids reached over $5 \mathrm{~m}$ in total length during the lower Toarcian (e.g.

3736 Macrospondylus; Westphal, 1961); they continued to get bigger in the Middle and Late Jurassic,

3737 and into the Cretaceous (with Mac. rex hypothesized to be around $7.15 \mathrm{~m}$ in total length; Young

3738 et al., 2016). Teleosaurids remained smaller in every ecosystem in which they co-existed with 
3739 machimosaurids; only the taxa Mystriosaurus and Mycterosuchus came close to the body sizes of

3740 machimosaurids. It is possible that this difference in body size is related to territory, locomotor

3741 and thermoregulation performance, and food sources, as in modern crocodylians (Grigg et al.,

3742 1998; Elsworth, Seebacher \& Franklin, 2003).

3743

3744 Conclusions

3745 Despite an increase in morphological work within the past decade, the evolutionary relationships

3746 of teleosauroids are poorly understood and little studied, and thus their macroevolutionary

3747 patterns are rarely evaluated. One major issue is the genus Steneosaurus, which is often

3748 recovered as paraphyletic or polyphyletic in phylogenetic analyses. Following on our recent re-

3749 classification of Steneosaurus as a nomen dubium and an invalid genus (Johnson, Young \&

3750 Brusatte, 2020), we herein presented an in-depth phylogenetic evaluation of Teleosauroidea. We

3751 firstly proposed the following changes to teleosauroid nomenclature, as a direct result of the

3752 invalidity of Steneosaurus: seven new generic names (Plagiophthalmosuchus,

3753 Clovesuurdameredeor, Seldsienean, Charitomenosuchus, Proexochokefalos, Andrianavoay and

3754 Neosteneosaurus) and one new species (Indosinosuchus kalasinensis); and the resurrection of

3755 three historical genera (Macrospondylus, Aeolodon and Sericodon). Secondly, we described 38

3756 new characters and 19 additional characters that are important and distinctive in teleosauroid

3757 morphology and discussed how these characters differ between taxa. Thirdly, we listed the

3758 results of the phylogenetic analyses based on our updated $\mathrm{H}+\mathrm{Y}$ data matrix, containing 153 taxa

3759 (including 27 teleosauroids) and 502 osteological characters. Our results showed that both

3760 parsimony and Bayesian topologies are relatively consistent with one another. Next, we propose

Peer) reviewing PDF | (2020:03:47227:2:0:NEW 29 Jul 2020) 
3761 and define the following taxonomic clades: the families Teleosauridae (re-defined) and

3762 Machimosauridae, and the subfamilies Aeolodontinae and Machimosaurinae (which includes

3763 Machimosaurini). Finally, we evaluated the ecomorphology and distribution of teleosauroids,

3764 based on our new phylogeny. Teleosauridae and Machimosauridae are morphologically distinct,

3765 with differing biogeographic distributions (Teleosauridae is Laurasian and Machimosauridae is

3766 Sub-Boreal European-Gondwanan), habitat preferences and feeding strategies. The phylogeny

3767 infers that the teleosaurids were overall more phenotypically plastic than machimosaurids, with

3768 an east-Asian freshwater clade, a nascent pelagic clade, and a heavily armoured clade;

3769 machimosaurids were dominant in terms of abundance and dispersal, with a linear pattern of

3770 morphological changes. By evaluating our updated phylogeny, it is clear that teleosauroids were,

3771 in terms of morphology, ecology and geography, more diverse than previously thought.

3772

\section{Acknowledgements}

3774 We sincerely thank R. Butler (BIRUG), M. Williams (BRLSI), M. Riley (CAMSM), I.

3775 Werneburg (GPIT), A. Gehler (GZG), A. Folie (IRSNB), X. Xing and L. Zhang (IVPP), A.

3776 Richter (LMH), G. Garcia, F. Guy and P. Vignaud (LPP), O. Mateus (MG and ML), M. Wilmsen

3777 (MMG), R. Allain (MNHN), B. Thuy and R. Weis (MNHNL), L. Póvoas (MUHNAC), P.

3778 Barrett and S. Maidment (NHMUK), U. Göhlich (NHMW), C. Howell (NMW), A. Smith

3779 (NOTNH), T. Mörs (NZM), J. Dridi (ONM), E. Howlett and H. Ketchum (OUMNH), G. Wass

3780 (PETMG), K. Lauprasert (PRC), R. Brocke (SMF), R. Kasma (SMHM), E. Maxwell and R.

3781 Schoch (SMNS), and S. King (YORM) for access to collections. M.M. Johnson would like to

3782 thank S. Sachs (NAMU), E. Wilberg (SBU), Y. Lepage, W. Simpson (FMNH), V. Lamarque 
3783 (MMT), L. Schöllmann (LWL), B. Erkt (NM), U. Menkveld-Gfeller (NMBE), G. Wahlefeld

3784 (NMR), D. Foffa (NMS), M. Manabe (NMNSJ), A. Sennikov (PIN), and B. Kear (PMU) for

3785 additional photos and/or information on specimens, as well as M. Andrade, A. Brignon, C.

3786 Brochu, P. Havlik, R. Irmis, S. Jouve, J. Liston, F. Ortega, E. Puértolas, M. Rabi, E. Saupe, G.

3787 Sobral and L. Steel for in-depth, thoughtful discussion. We also thank G. Alemu, J. Anderson, G.

3788 Antell, J. Anquetin, A. Averianov, J.-P. Billon-Bruyat, E. Buffetaut, L. Cavin, S. Chapman, J.

3789 Choiniere, L. ChunChi, A. Clark, L. Costeur, C. Dal Sasso, I. Danilov, H. Dermot, K. Dollman,

3790 D. Đurić, B. Eichner-Grünbeck, P. Ensom, Z. Erasmus, D. Evans, M. Evans, J. Galpin, R.Hauff,

3791 J. Hoeflinger, P. Holroyd, S. Hood, Y. Hongyu, J. Hornung, S. Hua, S. Humphrey, C. Klug, N.

3792 Knötscke, M. Križnar, D. Lomax, T. Lyson, R. Marchant, C. Mehling, A. Millhouse, J.

3793 Nurnberg, R. Osbourne, N. Oyal, D. Pickering, L. Picot, K. Shelburn, W. Simkiss, M. Simms, N.

3794 Spassov, G. Storrs, K. Strang, S. Thüring, V. Vadja, P. Vignaud, S. Zaurbekov and D. Zelenitsky

3795 for additional information and references, and helpful advice. M.M. Johnson would like to thank

3796 Z. Kynigopoulou (University of Edinburgh) and P. Webb-Davies (Bangor University) for help

3797 with Greek and Old English translations, respectively. The authors would like to sincerely thank

3798 M. Laurin, A. Selles and an anonymous reviewer for their helpful comments and feedback,

3799 which greatly improved the manuscript. This work was supported by the Natural Sciences and

3800 Engineering Council of Canada [grant number PGSD3-487581-2016] and SYNTHESYS Project

3801 [FR-TAF-6577] to M.M. Johnson; and a Leverhulme Trust Research Project [grant number

3802 RPG-2017-167] to S. Brusatte and M. Young.

3803

3804 References 
3806 (Crocodyliformes, Alligatoridae) from the Upper Miocene Urumaco Formation, Venezuela.

3807 Journal of Systematic Palaeontology 4: 221-232.

3808

Amir A, Keselman D. 1997. Maximum agreement subtree in a set of evolutionary trees:

3809 metrics and efficient algorithms. SIAM Journal on Computing 26: 1656-1669.

3810

3811

Andrade MB, Bertini RJ. 2008. Morphology of the dental carinae in Mariliasuchus

3812 amarali (Crocodylomorpha, Notosuchia) and the pattern of tooth serration among basal

3813 Mesoeucrocodylia. Arquivos do Museu Nacional, Rio de Janeiro 66: 63-82.

3814 Andrade MB, Young MT, Desojo JB, Brusatte SL. 2010. The evolution of extreme 3815 hypercarnivory in Metriorhynchidae (Mesoeucrocodylia: Thalattosuchia): evidence from 3816 microscopic denticle morphology and a new tri-faceted Kimmeridgian tooth from Germany. 3817 Journal of Vertebrate Paleontology 30: 1451-1465.

3818 Andrade MB, Edmonds R, Benton MJ, Schouten R. 2011. A new Berriasian species of 3819 Goniopholis (Mesoeucrocodylia, Neosuchia) from England, and a review of the genus. 3820 Zoological Journal of the Linnean Society 163: 66-108.

3821 Andrews CW. 1909. XXXVIII - on some new Steneosaurs from the Oxford Clay of 3822 Peterborough. Annals and Magazine of Natural History 3: 299-308. 
3825 Bacheley C. 1778a. Nature du sol du Mont de Sainte Catherine à l'est-sud-est de Rouen

3826 et ses différentes productions d'histoire naturelle.Lépecq de la Clôture, L. In: Collection

3827 d'observations sur les mala-dies et constitutions épidémiques. Imprimerie privilégiée, Rouen,

3828 pp.215-216.

3829

Bacheley C. 1778b. Notice des pétrifications \& autres faits d'histoire natu-relle qui se

3830 trouvent le long [des] côtes du Pays d'Auge. Lépecq de la Clôture, L. In: Collection

3831 d'observations sur les maladies et constitu-tions épidémiques. Imprimerie privilégiée, Rouen, pp.

$3832 \quad 357-359$.

3833

Ballell A, Moon BC, Porro LB, Benton MJ, Rayfield EJ. 2019. Convergence and

3834 functional evolution of longirostry in crocodylomorphs. Palaeontology: DOI:

$383510.1111 /$ pala.12432.

3836

Bardet N, Hua S. 1996. Simolestes nowackianus HUENE, 1938 from the Late Jurassic of

3837 Ethiopia is a teleosaurid crocodile, not a pliosaur. Neues Jahrbuch fur Geologie und

3838 Palaontologie Monatshefte 1996: 65-71.

3839

Barrientos-Lara JI, Alvarado-Ortega J, Fernández MS. 2018. The marine crocodile

3840 Maledictosuchus (Thalattosuchia, Metriorhynchidae) from the Kimmeridgian deposits of

3841 Tlaxiaco, Oaxaca, southern Mexico. Journal of Vertebrate Paleontology 38: DOI:

$384210.1080 / 02724634.2018 .1478419$.

Benton MJ, Taylor MA. 1984. Marine reptiles from the upper Lias (Lower Toarcian,

3844 Lower Jurassic) of the Yorkshire Coast. Proceedings of the Yorkshire Geological Society 44:

$3845 \quad 399-429$. 
3852 Voorst, London, $475 \mathrm{pp}$.

3853 Bremer K. 1988. The limits of amino acid sequence data in angiosperm phylogenetic 3854 reconstruction. Evolution 42: 795-803. 3856 (1797-1874). Édité par l'auteur, Bourg-la-Reine, vi + 82 p., 16 pl. reptiles marins jurassiques des Vaches Noires. Geodiversitas 40: 43-68. 49-62. 
3866 für Naturgeschichte 8: 77-82.

3867 Bronzati M, Montefeltro FC, Langer. 2012. A species-level tree of Crocodyliformes. 3868 Historical Biology 24: 598-606.

3869 Brusatte SL, Carr TD. 2016. The phylogeny and evolutionary history of tyrannosauroid 3870 dinosaurs. Scientific Reports: DOI: 10.1038/srep20252. neurosensory anatomy of an Early Jurassic marine crocodylomorph: implications for crocodylian 3873 sinus evolution and sensory transitions. The Anatomical Record 299: 1551-1530.

3875 Bronn, 1841 (Crocodylia, Mesosuchia), du Toarcien d'Europe. Geobios 13: 783-786.

3877 crocodiliens mesosuchiens marins du Mesozoique. Comptes-Rendus du Congrès National des 3878 Sociétés Savantes - Section des Sciences 105: 11-22. mésosuchiens. Mémoires de la Société Géologique de France 60: 1-88. 
3885 dans le Kimméridgien de Villerville (Calvados). Bulletin trimestriel de la Société Géologique de 3886 Normandie et des Amis du Muséum du Havre, tome 71, fasc. 4: p. 19-24. (Oxfordien sup., Normandie). Comptes Rendus d'Académie des Sciences 2: 929-932. theropod from southern China. Geological Magazine 145: 745-748.

3892 Busack SD, Pandya S. 2001. Geographic variation in Caiman crocodilus and Caiman 3893 yacare (Crocodylia: Alligatoridae): systematic and legal implications. Herpetologica 57: 2943894312. near Whitby in Yorkshire. Philosophical Transactions of the Royal Society of London 50: 6883902691. 
3904 and the origin of tyrannosaurs. Philosophical Transactions of the Royal Society of London B 309:

$3905 \quad 395-460$.

3906

Claessens LPAM. 2004. Archosaurian respiration and the pelvic girdle aspiration

3907 breathing of crocodyliforms. Proceedings of the Royal Society of London B 271: 1461-1465.

3908

Clarac F, Souter T, Cornette R, Cubo J, de Buffrénil V. 2015. A quantitative assessment

3909 of bone area increase due to ornamentation in the Crocodylia. Journal of Morphology 276: 1183-

39101192.

3911

Clarac F, Goussard F, de Buffrénil V, Sansalone V. 2019. The function(s) of bone

3912

ornamentation in the crocodylomorph osteoderms: a biomechanical model based on a finite

3913 element analysis. Paleobiology 45: 182-200.

3914

Clark JM. 1994. Patterns of evolution in Mesozoic Crocodyliformes. Pp. $84-97$ in N. C.

3915 Fraser \& H. D. Sues (eds). In the shadows of dinosaurs: early Mesozoic tetrapods. London:

3916 Cambridge University Press.

3917

Congreve CR, Lamsdell JC. 2016. Implied weighting and its utility in palaeontological

3918 datasets: a study using modelled phylogenetic matrices. Palaeontology 59: 447-462.

3919

Cortes D, Larsson HCE, Maxwell EE, Parra Ruge ML, Patarroyo P, Wilson JA.2019. An

3920 Early Cretaceous teleosauroid (Crocodylomorpha: Thalattosuchia) from Colombia.

3921 Ameghiniana. doi:10.5710/AMGH.26.09. 2019.3269. 
3922 Crompton AW, Smith KK. 1980. A new genus and species from the Kayenta Formation

3923 (Late Triassic?) of Northern Arizona. In: Jacobs, L (ed.), Aspects of Vertebrate History Flagstaff:

3924 Museum of Northern Arizona Press, pp. 193-217.

3925 Currie PJ. 1995. New information on the anatomy and relationships of Dromaeosaurus 3926 albertensis (Dinosauria: Theropoda). Journal of Vertebrate Paleontology 15: 576-591.

3927 Cuvier G. 1800. Sur une nouvelle espèce de crocodile fossile. Bulletin de la Sociéte 3928 philomatique de Paris 2: 159.

3929

Cuvier G. 1808. Sur les ossemens fossiles de crocodiles, et particulièrement sur ceux des

3930 environs du Havre et de Honfleur, avec des remarques sur les squelettes des sauriens de la

3931 Thuringe. Annales du Muséum d'histoire naturelle 12: 73-110.

3932 Cuvier G. 1812. Recherches sur les ossemens fossiles de quadrupèdes ou l'on rétablit les 3933 caractères de plusieurs espèces d'animaux que les revolutions du globe paroissent avoir 3934 détruites. Paris, Déterville, 1812, Tome IV.

3936 plusieurs animaux dont les révolutions du globe ont détruit les espèces. Vol. V, 2e partie., 2e 3937 édition G. Dufour et E. d'Ocagne, Paris, 547 p., 33 pl. 
3940 De Lapparent A. 1955. Étude paléontologique des vertébrés du Jurassique d'El Mers 3941 (Moyen Atlas) [Paleontological study of the Jurassic veretebrates from El Mers (Middle Atlas)]. 3942 Notes et Mémoires du Service Géologique du Marooc 124: 1-36.

3944 the Middle Jurassic of Tunisia. Geobios 56: 95-106.

3946 comments on the stratigraphic context of the Douiret Formation. Annales de Paléontologie:

3947 https://doi.org/10.1016/j.annpal.2020.102424.Efimov MB. 1982. New fossil crocodiles from the 3948 territory of the USSR. Paleontologicheskii Zhurnal 2: 146-150. crocodiles (Crocodylus porosus): effects of body size and temperature. Journal of Herpetology 3954 37: 363-368.

3956 Steneosaurus megistorhynchus (GEOFF.) trouvée à Allemagne et comparaison de cette espèce 3957 avec le Teleosaurus larteti (DESL.). Bulletin de la Societé Linnéenne de Normandie 10: 80-85. 

193-223.

3962 Téléosauriens, désigné sous le nom de complémentaire. Bulletin de la Societé Linnéenne de 3963 Normandie, Caen, série 2, 2: 381-473. cadomensis. Bulletin de la Societé Linnéenne de Normandie, Caen, série 2, 2: 381-473. Caen, se rapprochant du premier type créé par Geoffroy Saint-Hilaire, sous le nom de Teleosaurus cadomensis. Bulletin de la Société Linnéenne de Normandie Caen, Année 1866, 2:

3969 326-352.

3972 Linnéenne de Normandie, série 2, 3: 124-221. 
3977 crocodiles in the Middle and Upper Jurassic of Tunisia. Neues Jahrbuch fur Geologie und 3978 Palaontologie Monatshefte 8: 465-476.

3979

Feranec RS. 2007. Ecological generalization during adaptive radiation: evidence from

3980 Neogene mammals. Evolutionary Ecology Research 9: 555-577.

3981

Fischer V, Clément A, Guiomar M, Godefroit P. 2011. The first definite record of a

3982 Valanginian ichthyosaur and its implications on the evolution of post-Liassic Ichthyosauria.

3983 Cretaceous Research 32: 155-163.

3984

Foffa D, Young MT. 2014. The cranial osteology of Tyrannoneustes lythrodectikos

3985 (Crocodylomorpha: Metriorhynchidae) from the Middle Jurassic of Europe. PeerJ 2: e608; DOI $398610.7717 /$ peerj.608.

Foffa D, Young MT, Brusatte SL. 2015. Evidence of macrophagous teleosaurid 3988 crocodylomorphs in the Corallian Group (Oxfordian, Late Jurassic) of the UK. PeerJ 3: e1497. 3989 DOI:10.7717/peerj.1497.

3990

Foffa D, Johnson MM, Young MT, Steel L, Brusatte SL. 2019. Revision of the Late 3991 Jurassic deep-water teleosauroid crocodylomorph Teleosaurus megarhinus Hulke, 1871, and 3992 evidence of pelagic adaptations in Teleosauroidea. PeerJ 7: 6646.

3994 Juraformation. Jahreshefte des Vereins für vaterländische Naturkunde in Württemberg 57: 4093995418. 
3997 Berücksichtigung von Dacosaurus und Geosaurus. Palaeontographica 49: 1-72.

3998

Gasparini Z, Fernández M. 2005. Jurassic marine reptiles in the Neuquén Basin. Pp. 279-

3999

294 in G. Veiga, L. Spalletti, E. Schwarz \& J. Howell (eds) The Neuquén Basin: A Case Study in

4000

Sequence Stratigraphy and Basin Dynamics, Special Publication 252. Geological Society of

4001 London, London.

4002

Gasparini Z, Pol D, Spalletti LA. 2006. An unusual marine crocdyliform from the

4003

Jurassic-Cretaceous boundary of Patagonia. Science 311: 70-73.

4004

Geoffroy Saint-Hilaire E. 1825. Recherches sur l'organisation des gavials, sur leurs

4005

affinités naturelles desquelles résulte la nécessité d'une autre distribution générique: Gavialis,

4006

Teleosaurus, Steneosaurus; et sur cette question, si les gavials (Gavialis), aujourd'hui répandus

4007 dans les parties orientales de l'Asie, descendent, par voie non interrompue de génération, des

4008 gavials antidiluviens, soit des gavials fossiles, dits crocodiles de Caen (Teleosaurus), soit des

4009 gavials fossiles du Havre et de Honfleur (Steneosaurus). Mémoires du Muséum national

4010 d'histoire naturelle 12: 97155, pl.5-6.

4011

Geoffroy Saint-Hilaire E. 1831. Recherches sur de grands sauriens trouvés al'état fossile

4012

aux confins maritimes de la Basse-Normandie, attribués d'abord au Crocodile, puis déterminés

4013 sous les noms de Teleosaurus et Steneosaurus. Mémoires de l'Academie des sciences 12: 1138.

4014 George ID, Holliday CM. 2013. Trigeminal nerve morphology in Alligator

4015 mississippiensis and its significance for crocodyliform facial sensation and evolution. The

4016 Anatomical Record 296: 670-680. 
4017 Godefroit P, Vignaud P, Lieger A. 1995. Un Teleosauridae (Reptilia) du Bathonien

4018 superieur lorrain (France). Bulletin de la Société belge de Géologie, T 104: 91-107.

4019 Gold MEL. 2011. Cranial osteology and braincase morphometrics of Gavialis

4020 gangeticus: implications for crocodilian phylogenetics. Master's Thesis, University of Iowa,

4021 USA. http://ir.uiowa.edu/etd/2504.

4022 Goloboff PA. 2014. Extended implied weighting. Cladistics 30: 260-272.

4023 Goloboff PA, Farris JS, Nixon KC. 2008. TNT, a free program for phylogenetic analysis.

4024 Cladistics 24: 774-786.

4025 Goloboff PA, Catalano SA. 2016. TNT version 1.5, including a full implementation of 4026 phylogenetic morphometrics. Cladistics 32: 221-238.

4027

Grand A, Corvez A, Duque Velez LM, Laurin M. 2013. Phylogenetic inference using

4028 discrete characters: performance of ordered and unordered parsimony and of three-item

4029 statements. Biological Journal of the Linnean Society 110: 914-930.

4030 Grigg G, Seebacher F. 2001 Crocodilian thermal relations. In: Crocodilian biology and 4031 evolution (eds GC Grigg, F Seebacher, CE Franklin), pp. 297-309. Chipping Norton, UK: Surrey 4032 Beatty.

4033 Grigg G, Seebacher F, Beard LA, Morris D. 1998. Thermal relations of large crocodiles, 4034 Crocodylus porosus, free-ranging in a naturalistic situation. Procedings of the Royal Society of 4035 London B 265: 1793-1799. 
4036 Grigoriev DV. 2014. Giant Mosasaurus hoffmanni (Squamata, Mosasauridae) from the

4037 Late Cretaceous (Maastrichtian) of Penza, Russia. Proceedings of the Zoological Institute 318:

4038 148-167.

4039 Hantzpergue P, Lafaurie G. 1983. Le kimmeridgien Quercynois; Un complement

4040 biostratigraphique du Jurassique supérieur d'Aquitaine. Géobios 16: 601-609.

4041 Hay OP. 1930. Second Bibliography and Catalogue of the Fossil Vertebrata of North

4042 America. Washington, D.C., Carnegie Institution of Washington, 916 pp.

4043 Hendrickx C, Mateus O, Araújo R. 2015. A proposed terminology of theropod teeth

4044 (Dinosauria, Saurischia). Journal of Vertebrate Paleontology 35: DOI:

$4045 \quad 10.1080 / 02724634.2015 .982797$.

$4046 \quad$ Herrera Y, Fernández MS, Gasparini Z. 2013. Postcranial skeleton of Cricosaurus

4047 araucanensis (Crocodyliformes: Thalattosuchia): morphology and palaeobiological insights,

4048 Alcheringa: Australasian Journal of Palaeontology 37: 285-298.

4049

Holder MT, Sukumaran J, Lewis PO. 2008. A justification for reporting the majority-rule 4050 consensus tree in Bayesian phylogenetics. Systematic Biology 57: 814-821.

4051 Holliday CM. 2006. Evolution and function of the jaw musculature and adductor 4052 chamber of archosaurs (crocodilians, dinosaurs and birds). Ph.D. dissertation, Ohio University, 4053 Athens, Ohio, 325 pp. 
4054 Holliday CM, Nesbitt SJ. 2013. Morphology and diversity of the mandibular symphysis 4055 of archosauriforms. Geological Society London, Special Publications 379: 1-18 DOI $405610.1144 / \mathrm{SP} 379.2$.

4057

4058 4059

4060

4061

4062

4063

4064 4065

4066 4067

4068 4069

4070 4071

Hua S. 1996. Reexamen du Machimosaurus cf. hugii des carrieres d'Haudainville

(Meuse, Est de la France): contribution a l'etude du genre Machimosaurus Meyer, 1838. Bulletin trimestriel de la Société Géologique de Normandie et des Amis du Muséum du Havre 83: 11-16.

Hua S. 1999. Le crocodilien Machimosaurus mosae (Thalattosuchia, Teleosauridae) du Kimmeridgien du Boulonnais (Pas de Calais, France). Palaeontographica A 252: 141-170.

Hua S, Buffetaut E. 1997. Crocodylia. In: Callaway JM, Nicholls EL, eds. Ancient marine reptiles. San Diego: Academic Press, 357-374.

Huelsenbeck JP, Ronquist F. 2001. MrBayes: Bayesian inference of phylogenetic trees. Bioinformatics 17: 754-755.

Huelsenbeck JP, Ronquist F, Nielsen R, Bollback, JP. 2001. Evolution—Bayesian inference of phylogeny and its impact on evolutionary biology. Science 294: 2310-2314.

von Huene F. 1926. Die Saurierfauna des Portlandkalkes von Solothurn. Eclogae

Geologicae Helvetiae 19: 584-603.

von Huene F. 1927. Beitrag zur Kenntnis mariner Mesozoischer Wirbeltierre in Argentinien. Centralblatt für Mineralogie, Geologie und Paläontologie Abteilung B 1: 22-29.

Peer] reviewing PDF | (2020:03:47227:2:0:NEW 29 Jul 2020) 
von Huene F. 1938. Ein Pliosauride aus Abessinien. Zentralblatt für Mineralogie,

4073 Geologie und Paläontologie 1938: 370-376.

4074 Hulke JW. 1871. Note on a Fragment of a Teleosaurian snout from Kimmeridige Bay,

4075 Dorset. Quarterly Journal of the Geological Society of London 17: 442-443.

4076 Hulke JW. 1877. Note on a gavial skull from the Cornbrash of Closworth. Proceedings of 4077 the Dorset Natural History and Antiquarian Field Club, i. p. 29, pl. I.

4078 Jäger CF. 1828. Über die fossile Reptilien, welche in Württemberg aufgefunden worden 4079 sind. Stuttgart: J. B. Metzler, 48 pp.

4080 Jagt JWM, Lindgren J, Machalski M, Radwański A. 2005. New records of the tylosaurine 4081 mosasaur Hainosaurus from the Campanian-Maastrichtian (Late Cretaceous) of central Poland. 4082 Netherlands Journal of Geosciences 84: 303-306.

4083 Jenkyns HC, Schouten-Huibers L, Schouten S, Sinninghe Damsté JS. 2012. Warm 4084 Middle Jurassic-Early Cretaceous high-latitude sea-surface temperatures from the Southern 4085 Ocean. Climate of the Past 8: 215-226.

4086 Johnson MM, Young MT, Steel L, Lepage Y. 2015. Steneosaurus edwardsi 4087 (Thalattosuchia, Teleosauridae), the largest known crocodylomorph of the Middle Jurassic. 4088 Biological Journal of the Linnean Society 115: 911918. Johnson MM, Young MT, Steel L, Foffa D, Smith AS, Hua S, Havlik P, Howlett EA, 4090 Dyke G. 2017. Re-description of 'Steneosaurus' obtusidens Andrews, 1909, an unusual 
4091 macrophagous teleosaurid crocodylomorph from the Middle Jurassic of England. Zoological

4092 Journal of the Linnean Society 1: 1-34. DOI:10.1093/zoolinnean/zlx035.

4093 Johnson MM, Young MT, Brusatte SL, Thuy B, Weis R. 2018. A catalogue of

4094 teleosauroids (Crocodylomorpha: Thalattosuchia) from the Toarcian and Bajocian (Jurassic) of

4095 southern Luxembourg. Historical Biology: DOI:10.1080/08912963.2018.1427090.

4096 Johnson MM, Young MT. Brusatte SL. 2019. Re-description of two contemporaneous

4097 mesorostrine teleosauroids (Crocodylomorpha, Thalattosuchia) from the Bathonian of England,

4098 and insights into the early evolution of Machimosaurini. Zoological Journal of the Linnean

4099 Society zlz037: https://doi.org/10.1093/zoolinnean/zlz037.

4100 Johnson MM, Young MT, Brusatte SL. 2020. Emptying the Wastebasket: a historical and

4101 taxonomic revision of the Jurassic crocodylomorph Steneosaurus. Zoological Journal of the

4102 Linnean Society zlaa027: https://doi.org/10.1093/zoolinnean/zlaa027.

4103 Joleaud L. 1928. Sur un crane de crocodilian sténéosaure découvert dans des argiles du

4104 Kimmeridgien supérieur d'Octeville-sur-Mer, près du Havre. Association Française pour

4105 l'Avancement des Sciences Paris 52: 331-332.

4106 Jouve S. 2009. The skull of Teleosaurus cadomensis (Crocodylomorpha; Thalattosuchia), 4107 and phylogenetic analysis of Thalattosuchia. Journal of Vertebrate Paleontology 29: 88-102.

4108 Jouve S, Mennecart B, Douteau J, Jalil N-E. 2016. The oldest durophagous teleosaurid

4109 (Crocodylomorpha, Thalattosuchia) from the Lower Bathonian of Central High Atlas, Morocco.

4110 Palaeontology 59: 863-876. 
4111 Karl HV, Gröning E, Brauckmann C, Knötschke N. 2008. First remains of the head of

4112 Steneosaurus (Crocodylomorpha: Teleosauridae) from the late Jurassic of Oker (Lower Saxony,

4113 Germany). Studia Geológica Salmanticensia 41: 187-201.

4114 Kaup, JJ. 1834. Versuch einer Eintheilung der Saugethiere in 6 Stämme und der

4115 Amphibien in 6 Ordnungen. Isis 3: 311-315.

4116 Khadkikar AS. 1996. Breakup of Gondwanaland and the Jurassic record of the Kachchh

4117 Basin, Gujarat, Western India. Current Science 70: 1093-1096.

4118 Klinkhamer AJ, Wilhite DR, White MA, Wroe S. 2017. Digital dissection and three-

4119 dimensional interactive models of limb musculature in the Australian estuarine crocodile

4120 (Crocodylus porosus). PLoS ONE 12: e0175079. https://doi.org/10.1371/journal.pone.0175079.

4121 Korte C, Hesselbo SP, Ulmann CV, Dietl G, Ruhl M, Schweigert G, Thibault N. 2015.

4122 Jurassic climate mode governed by ocean gateway. Nature Communications: DOI:

$412310.1038 /$ ncomms 10015.

4124 Krebs B. 1967. Der Jura-Krokodilier Machimosaurus H. v. Meyer. Paläontologische

4125 Zeitschrift 41: 46-59.

4126

Krebs B. 1968. Le crocodilien Machimosaurus. Servico Geólogico Portugal (Nova Série)

4127 14: 21-53.

4128

Kuhn O. 1936. Crocodilia. In: Fossilium Catalogus, Animalia. W. Quenstedt Ed, Berlin,

4129 1, 75: 1-144. 
4131 d'Allemagne, a’un quart de lieue de Cean. Annales Générales des Sciences Physiques 3: 1604132164.

4134 (Crocodyliformes, Thalattosuchia): testing its homology within a phylogenetic framework. 4135 Journal of Vertebrate Paleontology 32: 490-494.

4137 integumentary sensory organs in crocodilians. The Journal of Experimental Biology 215: 421741384230. vertébrés. Bulletin de la Société Géologique de Normandie 12: 65-67, pl. XIII.

4142 anatomique, géologique et historique des fossiles présentés à l'exposition Les Crocodiliens 4143 fossiles de Normandie (6 novembre-14 decembre 2008). Bulletin de la Société Géologique de 4144 Normandie et des Amis du Muséum du Havre 95: 5-152.

4146 morphological character data. Systematic Biology 50: 913-925. 
4150 [Chinese 69; English 70].

4151 Lindgren J. 2005. Dental and vertebral morphology of the enigmatic mosasaur

4152 Dollosaurus (Reptilia, Mosasauridae) from the lower Campanian (Upper Cretaceous) of southern

4153 Sweden. Bulletin of the Geological Society of Denmark 52: 17-25.

4154 Magnusson WE, da Silva EV, Lima AP. 1987. Diets of Amazonian crocodilians. Journal 4155 of Herpetology 21: 85-95.

4157 (Crocodylia, Thalattosuchia) from the Kimmeridgian of Germany. Fossil Record 16: 179-196.

4159 (Crocodylomorpha, Thalattosuchia). Comptes Rendus Palevol 14: 305-310. environment. Paleobiology 42: 143-156.

$416610.1080 / 02724634.2018 .1549059$.

4167 Mercier J. 1933. Contribution à l'étude des Métriorhynchidés (crocodiliens). Annales de 4168 Paléontologie 22: 99-119. 
4169 von Meyer H. 1831. Neue fossile Reptilien, aus der Ordnung der Saurier. Nova Acta 4170 Academiae Leopddino-Carolinae, Curios 15: 171-200.

4171 von Meyer H. 1832. Palaeologica zur Geschichte der Erde und ihrer Geschöpfe.

4172 Siegmund Schmerber, Franfurt am Main (xii +560 p.).

4173 von Meyer CEH. 1837. Mittheilungen, an Professor Bronn gerichtet. Neues Jahrbuch für 4174 Mineralogie, Geognosie, Geologie und Petrefaktenkunde 1837: 557-562. von Meyer CEH. 1838. Mittheilungen, an Professor Bronn. Neues Jahrbuch für 4176 Mineralogie, Geognosie, Geologie und Petrefaktenkunde 1838: 413-418. von Meyer H. 1845. System er fossilen Saurier, Mittheilung an Prof. Bronn gerichtet. 4178 Neues Jahrbuch für Mineralogie, Geologie und Paläontologie Stuttgart: 689-691.

4179 Mishra B, Thines M. 2014. siMBa - a simple graphical user interface for the Bayesian 4180 phylogenetic inference program MrBayes. Mycol Progress 13: 1255-1258.

4182 Steneosaurus heberti. Bulletin de la Société Géologique de France 3 4: 342-348.

4183 Morton C, Wooller Mr. 1758. A description of the fossil skeleton of an animal found in 4184 the alum rock near Whitby. Philosophical Transactions (1683-1775) 50: 786-790.

4186 Phylogenetics and Evolution 31: 780-782. 
4188 211-213.

4190 thalattosuchians (Mesoeucrocodylia) from the Liassic of Central Europe. Unpublished PhD

4191 thesis, Universität Mainz, Germany.

4192

Nesbitt SJ. 2011. The early evolution of Archosauria: relationships and the origin of 4193 major clades. Bulletin of the American Museum of Natural History 352: 1-292.

4195 baroni (n. sp.); with a reference to some post Tertiary vertebrate remains from the same country 4196 recently acquired by the British Museum (Natural History). The Geological Magazine 10: 1934197 198, pl. 9. crocodyliform from the lower Jurassic (Toarcian) of Hungary, with further evidence of the

4200 mosaic acquisition of marine adaptations in Metriorhynchoidea. PeerJ 6: e4668. Kotah. Quarterly Journal of the Geological Society of London 8: 233. 
4206 Phansalkar VG, Sudha G, Khadkikar AS. 1994. Giant marine reptilian skulls from the

4207 Jurassic of Kachchh, Gujarat. Current Science 67: 460-461.

4208 Phillips J. 1871. The Geology of Oxford and the Valley of the Thames. Clarendon Press,

4209 Oxford, 523 pp.

4210 Phizackerley PH. 1951. A revision of the Teleosauridae in the Oxford University

4211 Museum and the British Museum (Natural History). Annals and Magazine of Natural History 12:

$4212 \quad 1169-1192$.

4213 Pierce SE, Angielczyk KD, Rayfield EJ. 2009. Morphospace occupation in

4214 thalattosuchian crocodylomorphs: skull shape variation, species delineation and temporal

4215 patterns. Palaeontology 52: 1057-1097.

4216 Platt SG, Sovannara H, Kheng L, Stuart BL, Walston J. 2006. Crocodylus siamensis

4217 along the Sre Ambel River, southern Cambodia: habitat, nesting, and conservation.

4218 Herpetological Natural History 9: 183-188.

4219

Pol D, Apesteguia S. 2005. New Araripesuchus remains from the early Late Cretaceous

4220 (Cenomanian-Turonian) of Patagonia. American Museum Novitiates 3490: 1-38.

4221

Pol D, Gasparini Z. 2009. Skull anatomy of Dakosaurus andiniensis (Thalattosuchia:

4222 Crocodylomorpha) and the phylogenetic position of Thalattosuchia. Journal of Systematic

4223 Palaeontology 7: 163-197.

4224 Prasad GVR, Broin FL. 2002. Late Cretaceous crocodile remains from Naskal (India):

4225 comparisons and biogeographic affinities. Annales de Paléontologie 88: 19-71. 
4226

Prieto-Márquez A. 2010. Global phylogeny of Hadrosauridae (Dinosauria: Ornithopoda)

4227 using parsimony and Bayesian methods. Zoological Journal of the Linnean Society 159: 435-

4228502.

4229

Purvis A, Gittleman J, Brooks T. 2005. Phylogeny and Conservation. Cambridge

4230 University Press, Cambridge, 431 pp.

4231

Read MA, Miller JD, Bell IP, Felton A. 2004. The distribution and abundance of the

4232 estuarine crocodile, Crocodylus porosus, in Queensland. Wildlife Research 31: 527-534.

4233

Rebêlo GH, Lugli L. 2001. Distribution and abundance of four caiman species

4234 (Crocodylia: Alligatoridae) in Jaú National Park, Amazonas, Brazil. Revista de biología tropical 4235 49: 1096-1109.

4236

Rees PM, Ziegler AM, Valdes PJ. 2000. Jurassic phytogeography and climates: new data 4237 and model comparisons. In: Huber BT, Macleod KG, Wing SL (eds) Warm climates in earth 4238 history. Cambridge: Cambridge University Press, pp 297-318.

4239

Rineau V, Grand A, Zaragüeta R, Laurin M. 2015. Experimental systematics: sensitivity

4240 of cladistic methods to polarization and character ordering schemes. Contributions to Zoology

4241 84: 129-148.

4242

Ristevski J, Young MT, Andrade MB, Hastings AK. 2018. A new species of

4243 Anteophthalmosuchus (Crocodylomorpha, Goniopholididae) from the Lower Cretaceous of the

4244 Isle of Wight, United Kingdom, and a review of the genus. Cretaceous Research 84: 340-383.

4245

Romer AS. 1956. Osteology of the reptiles. University Chicago Press, Chicago. 
4247 mixed models. Bioinformatics 19: 1572-1574.

4248 Ronquist F, Teslenko M, van der Mark P, Ayres DL, Darling A, Hohna S, Larget B, Liu

4249 L, Suchard MA, Huelsenbeck JP. 2012. MrBayes 3.2: efficient Bayesian phylogenetic inference 4250 and model choice across a large model space. Systematic Biology 61: 539-542.

4251 Ross JP. 1998. Crocodiles. Status survey and conservation action plan, 2 nd Edition.

4252 UICN/SSC. Crocodile Specialist Group. Gland, Switzerland and Cambridge, London.

4253 Sachs S, Johnson MM, Young MT, Abel P. 2019a. The mystery of Mystriosaurus Kaup,

4254 1834: redescribing the poorly known Early Jurassic teleosauroid thalattosuchians Mystriosaurus 4255 laurillardi Kaup, 1834 and Steneosaurus brevior Blake, 1876. Acta Palaeontologica Polonica 64: 4256565579.

4258 crocodylomorph Cricosaurus from the Upper Jurassic of southern Germany. Acta

4259 Palaeontologica Polonica 64: DOI https://doi.org/10.4202/app.00541.2018.

4262 parallel evolutionary trends in skull shape linked to longirostry. PLoS ONE 11: e0152453.

4263 Salisbury SW, Naish D. 2011. Crocodilians. In: Batten D, Lane PD. (eds). English

4264 Wealden fossils. Palaeontological Association, Aberystwyth, 305-369. 
4265 Sauvage H-E. 1872. Sur quelques espèces de Sténéosaures provenant des assises

4266 jurassiques supérieures de Boulogne-sur-mer. Bulletin de la Société philomathique de Paris:

$4267 \quad 178-180$.

4268 Sauvage H-E. 1874. Mémoire sur les dinosauriens et les crocodiliens des terrains

4269 jurassiques de Boulogne-sur-Mer. Mémoires de la Société Géologique de France 10: 1-58.

4270 Sauvage H-E. 1896. Les crocodiliens et les dinosaurs des terrains mésozoïques du

4271 Portugal. Bulletin de la Société Géologique de France, Paris 24: 46-48.

4272

Sauvage H-E. 1897-98. Vertebres fossiles du Portugal: contributions a l'etude des

4273 Poissons et des reptiles du jurassique et du cretacique. Direction des travaux geologiques du

4274 Portugal 1: 1-47.

4275 Sauvage H-E, Liénard F. 1879. Memoire sur le genre Machimosaurus. Mémoires de la

4276 Société Géologique de France, 3ème série 4: 1-31.

4277

Saville É. 1876. Note sur un tête de Teleosaurus trouvée à Bléville. Bulletin de la Société

4278 géologique de Normandie 3: 52-57.

4279

Schaefer K, Püntener C, Billon-Bruyat J-P. 2018. Vertébrés mésozoïques-Crocodiliens.

4280 Office de la culture-Paléontologie A16, Porrentruy. Catalogues du patrimoine paléontologique

4281 jurassien-A16, $184 \mathrm{pp}$.

4282

Scheyer TM, Hutchinson JR, Strauss O, Delfino M, Carrillo-Briceño JD, Sánchez R,

4283 Sánchez-Villagra MR. 2019. Giant extinct caiman breaks constraint on the axial skeleton of

4284 extant crocodylians. eLife 8:e49972. DOI: https://doi.org/10.7554/eLife.49972. 
4285 Seeley HG. 1880. Note on the cranial characters of a large teleosaur from the Whitby

4286 Lias preserved in the Woodwaridan Museum of the University of Cambridge, indicating a new

4287 species, Teleosaurus eucephalus. Quarterly Journal of the Geological Society of London 36 :

$4288 \quad 627-634$.

4289

Seidel MR. 1979. The osteoderms of the American alligator and their functional

4290 significance. Herpetologica 35: 375-380.

4291 Sereno PC, Arcucci AB. 1990. The monophyly of crurotarsal archosaurs and the origin of

4292 bird and crocodile ankle joints. Neues Jahrbuch für Geologie und Paläontologie Abhandlungen

4293 180: 21-52.

4294

Slater GJ. 2013. Phylogenetic evidence for a shift in the mode of mammalian body size

4295 evolution at the Cretaceous-Palaeogene boundary. Methods in Ecology and Evolution 4: 734-

4296744.

4297

Smith MA. 1931. The Fauna of British India, Ceylon and Burma. Taylor \& Francis,

4298 London, United Kingdom.

4299

Soares D. 2002. An ancient sensory organ in crocodilians. Nature 417: 241-242.

4300

von Sömmerring ST. 1814. Über den Crocodilus priscus oder über ein in Baiern

4301 versteinert gefundenes Krokodil, Gavial der Vorwelt. Denkschriften der Königlichen Akademie

4302 der Wissenschaften zu München Classe 4: 1-74.

4303

Stanley GD. 1988. The history of early Mesozoic reef communities: a three-step process.

4304 Palaios 3: 170-183. 
4307 Age of Dinosaurs in Russia and Mongolia, ed. MJ Benton, MA Shishkin, DM Unwin, EN 4308 Kurochkin, pp. 402-19. Cambridge: Cambridge Univ. Press.

4310 Schneider in northern Australia. Australian Wildlife Research 6: 347-359. 4313 Conservation Action Plan. 3rd ed. Crocodile Specialist Group. Australia.

4315 dos Serviços Geologicos de Portugal new serie 13: 66p.

4317 Manolis \& C. Stevenson, eds. Crocodiles: Status Survey and Conservation Action Plan, $3^{\text {rd }}$ 4318 Edition. Crocodile Specialist Group, Darwin. conservation priorities for the American crocodile. Biological Conservation 128: 25-36. 
4324 Vasconcelos WR, Hrbeck T, da Silveira R, de Thoisy B, Dos Santos Ruffei LAA, Farias

4325 IP. 2008. Phylogeographic and conservation genetic analysis of the Black caiman (Melanosuchus 4326 niger). Journal of Experimental Zoology 309A: 600-613.

4327 Vickaryous MK, Hall BK. 2008. Development of the dermal skeleton in Alligator 4328 mississippiensis (Archosauria, Crocodylia) with comments on the homology of osteoderms. 4329 Journal of Morphology 269: 398-422.

4330 Vignaud P. 1995. Les Thalattosuchia, crocodiles marins du Mésozoique: Systématique 4331 phylogénétique, paléoécologie, biochronologie et implications paléogéographiques.

4332 Unpublished $\mathrm{PhD}$ thesis, Université de Poitiers.

4333 Vignaud P. 1998. Une nouvelle espece de Steneosaurus (Thalattosuchia, Teleosauridae)

4334 dans le Callovien du Poitou (France) et la systematique des Steneosaurus longirostres du

4335 Jurassique moyen d'Europe occidentale. Palaeovertebrata 27: 19-44.

4336

Walch JEI. 1796. Sammlung der Merkwürdigkeiten der Natur u.s.w. Nürnberg. Fol. P.

4337195.

4338

Webb GJW, Manolis SC, Brien ML. 2010. Saltwater crocodile Crocodylus porosus. In 4339 Crocodiles: Status Survey and Conservation Action Plan, $3^{\text {rd }}$ Edition (eds S.C. Manolis \& C.

4340 Stevenson), pp. 99-113. Crocodile Specialist Group, Darwin, Australia.

4341 Westphal F. 1961. Zur Systematik der deutschen und englischen Lias- Krokodilier. Neues 4342 Jahrbuch für Geologie und Paläontologie 113: 207-218. 
4343 Westphal F. 1962. Die Krokodilier des deutschen und englischen oberen Lias.

4344 Palaeontographica A 116: 23-118.

4345 Whitaker R, Basu D. 1983. The gharial (Gavialis gangeticus): a review. Journal of the 4346 Bombay Natural History Society 79: 531-548.

4347 Wilberg EW. 2015a. What's in an outgroup? The impact of outgroup choice on the 4348 phylogenetic position of Thalattosuchia (Crocodylomorpha) and the origin of Crocodyliformes. 4349 Systematic Biology 64: 621-637.

4351 the Middle Jurassic of Oregon and the evolutionary timing of marine adaptations in 4352 thalattosuchian crocodylomorphs. Journal of Vertebrate Paleontology 35: e902846.

4353 Wilberg EW. 2017. Investigating patterns of crocodyliform cranial disparity through the 4354 Mesozoic and Cenozoic. Zoological Journal of the Linnean Society 181: 189-208. habitat shifts in Crocodylomorpha. Scientific Reports: DOI: 10.1038/s41598-018-36795-1.

4358 (Mesoeucrocodylia: Thalattosuchia) from the Kimmeridgian (upper Jurassic) of Wiltshire, UK. 4359 Palaeontology 51: 1307-1333. 
Young CC. 1948. Fossil crocodiles in China, with notes on dinosaurian remains

4364 associated with the Kansu crocodiles. Vertebrata Palasiatica 28: 255-288.

Young MT. 2006. Evolution and taxonomic revision of the Mesozoic marine

4366 crocodyliforms Metriorhynchidae, a phylogenetic and morphometric approach. Unpublished

4367 MSc (Master of Science) thesis, University of London Imperial College, London, 140 pp.

$4370 \quad 26: 80-90$.

4372 (Thalattosuchia, Metriorhynchidae) from the Upper Jurassic of Bayern, Germany. Zoological 4373 Journal of the Linnean Society 157: 551-585.

4375 Metriorhynchoidea (Mesoeucrocodylia, Thalattosuchia), an integrated approach using geometric 4376 morphometrics, analysis of disparity, and biomechanics. Zoological Journal of the Linnean 4377 Society 158: 801-859.

4379 evolution in metriorhynchid crocodylomorphs: implications for species diversification and niche 4380 partitioning. Zoological Journal of the Linnean Society 163: 1199-1216. 
4383 of the metriorhynchoid crocodylomorph genera Dakosaurus and Plesiosuchus from the Late

4384 Jurassic of Europe. PLoS ONE 7: e44985 (doi: 10.1371/journal.pone.0044985).

4385 Young MT, Andrade MB, Brusatte SL, Sakamoto M, Liston J. 2013. The oldest known 4386 metriorhynchid super-predator: a new genus and species from the Middle Jurassic of England, 4387 with implications for serration and mandibular evolution in predacious clades. Journal of 4388 Systematic Palaeontology 11: 475-513.

4389 Young MT, Steel L. 2014. Evidence for the teleosaurid crocodylomorph genus 4390 Machimosaurus in the Kimmeridge Clay Formation (Late Jurassic) of England. Historical 4391 Biology 26: 472-479.

4393 JI, Havlik P, Lepage Y, Andrade MB. 2014. Revision of the Late Jurassic teleosaurid genus 4394 Machimosaurus (Crocodylomorpha, Thalattosuchia). Royal Society Open Science 1: 140222.

4395 Young MT, Beatty BL, Brusatte SL, Steel L. 2015a. First evidence of denticulated 4396 dentition in teleosaurid crocodylomorphs. Acta Palaeontologica Polonica 60: 661-671.

4398 JI, Havlik P, Lepage Y, Andrade MB. 2015b. Addendum to 'Revision of the Late Jurassic 4399 teleosaurid genus Machimosaurus (Crocodylomorpha, Thalattosuchia)'. Royal Society Open 4400 Science 2: 150024. 
$4401 \quad$ Young MT, Rabi M, Bell MA, Steel L, Foffa D, Sachs S, Peyer K. 2016. Big-headed

4402 marine crocodyliforms, and why we must be cautious when using extant species as body length

4403 proxies for long extinct relatives. Palaeontologia Electronica 19.3.30A: 1-14.

4404 Zanno LE, Drymala S, Nesbitt SJ, Schneider VP. 2015. Early crocodylomorph increases

4405 top tier predator diversity during rise of dinosaurs. Scientific Reports 5: 9276. DOI:

$440610.1038 /$ srep09276.

4407 


\section{Figure Legends}

4409 Figure 1. Recent strict consensus topologies focused on thalattosuchian phylogenetics, focusing

4410 on teleosauroids. Altered from (A) Mueller-Töwe (2006); (B) Jouve (2009); (C) Wilberg

4411 (2015b); (D) Johnson, Young \& Brusatte (2019); and (E) Martin et al. (2019).

4412 Figure 2. Plagiophthalmosuchus gracilirostris (Westphal, 1961) comb. nov., NHMUK PV OR

4413 14792, holotype. (A) Nearly complete skeleton, with close-up views of: (B) the skull, (B)

4414 forelimb and (D) pelvic area. Refer to abbreviations list. Scale bars: $10 \mathrm{~cm}(\mathrm{~A}-\mathrm{B})$ and $4 \mathrm{~cm}(\mathrm{C}-$ 4415 D).

4416 Figure 3. Mystriosaurus laurillardi Kaup, 1834, holotype HLMD V946-948 (A-C) and referred 4417 specimen NHMUK PV OR 14781 (D-F). (A, D) Dorsal, (B) left lateral, (C, F) ventral and (E) 4418 right lateral views. Refer to abbreviations list. Scale bars: $10 \mathrm{~cm}$. Photographs A to C provided 4419 by S. Sachs.

4420 Figure 4. Clovesuurdameredeor stephani (Hulke, 1877), comb. nov., NHMUK PV OR 49126, 4421 holotype. Skull in (A) dorsal, (B) ventral (palatal), (C) right and (D) left lateral views. Partial 4422 mandible in (E) dorsal view, and right retroarticular process in $(\mathrm{F})$ dorsal and $(\mathrm{G})$ right lateral 4423 views. Refer to abbreviations list. Scale bars: $10 \mathrm{~cm}(\mathrm{~A}-\mathrm{C})$ and $4 \mathrm{~cm}(\mathrm{E}-\mathrm{F})$.

4424 Figure 5. The Chinese teleosauroid previously referred to as Peipehsuchus (see Li, 1993), IVPP 4425 V 10098, holotype. Skull in (A) dorsal and (B) ventral (palatal) views. Refer to abbreviations 4426 list. Scale bars: $10 \mathrm{~cm}$. 
4427 Figure 6. Platysuchus multiscrobiculatus (Berckhemer, 1929) Westphal, 1961, SMNS 9930,

4428 holotype. (A) Nearly complete skeleton, with close-up views of (B) the skull, (C) forelimb, (D)

4429 trunk region and (E) hindlimb. Refer to abbreviations list. Not to scale.

4430 Figure 7. Teleosaurus cadomensis (Lamouroux, 1820), MNHN AC 8746, holotype. Partial skull

4431 in (A) dorsal, (B) ventral (palatal), (C) left lateral, (D) right lateral and (E) occipital views. Refer 4432 to abbreviations list. Scale bars: $5 \mathrm{~cm}$.

4433 Figure 8. Mycterosuchus nasutus Andrews, 1913, NHMUK PV R 2617, holotype. Skull in (A)

4434 dorsal and (B) ventral (palatal) views, and dentary in (C) dorsal view. Note the extremely rugose

4435 dorsal cranium. Refer to abbreviations list. Scale bars: $10 \mathrm{~cm}$.

4436 Figure 9. Aeolodon priscus (von Sömmering, 1814), (A-E) NHMUK PV R 1086, holotype and

4437 (F) MNHN.F.CNJ 78, referred specimen (modified from Figure 10 in Foffa et al. (2019)). (A)

4438 Partial skeleton with close-ups of (B) the skull, (C) hindlimb, (D) trunk region and (E) pelvic

4439 area. (F) Nearly complete skeleton. Scale bars: $10 \mathrm{~cm}$ (A) and $3 \mathrm{~cm}$ (B-E), (F) not to scale.

4440 Figure 10. Bathysuchus megarhinus (Hulke, 1871) Foffa et al., 2019. (A-D) NHMUK PV OR

4441 43086, holotype; (E-G) unnumbered LPP specimen. In (A, E) dorsal, (B) ventral, (C) right

4442 lateral, (D, F) left lateral and (G) occipital views. Refer to abbreviations list. Scale bars: $10 \mathrm{~cm}$.

4443 Figure 11. Sericodon jugleri von Meyer, 1845, referred specimens. (A) Tooth in lingual view

4444 (SMF R 4318) and (B) anterior mandible in dorsal view (LMH 16646). Refer to abbreviations

4445 list. Scale bars: $1 \mathrm{~cm}(\mathrm{~A})$ and $5 \mathrm{~cm}(\mathrm{~B})$. 
4446 Figure 12. Indosinosuchus potamosiamensis Martin et al., 2019, PRC-11, holotype. Skull and

4447 attached mandible in (A) dorsal and (B) ventral (palatal) views. Refer to abbreviations list. Scale 4448 bar: $10 \mathrm{~cm}$.

4449 Figure 13. Indosinosuchus kalasinensis, sp. nov., PRC-239. Skull and mandible in (A) dorsal 4450 and (B) right lateral views. Refer to abbreviations list. Scale bar: $10 \mathrm{~cm}$.

4451 Figure 14. Macrospondylus bollensis (Jäger, 1828). (A) MMG BwJ 595, holotype, partial 4452 postcranial skeleton. (B) Complete skeleton MMG BwJ 565. Refer to abbreviations list. Scale 4453 bars: $10 \mathrm{~cm}$.

4454 Figure 15. Seldsienean megistorhynchus (Eudes-Deslongchamps, 1866a), comb. nov., MMT 4455 P28-1, neotype. Skull in (A) dorsal, (B) ventral (palatal), (C) right lateral and (D) left lateral 4456 views. Mandible in (E) dorsal view. Refer to abbreviations list. Scale bars: $10 \mathrm{~cm}$. Photographs 4457 provided by V. Lamarque.

4458 Figure 16. Charitomenosuchus leedsi (Andrews, 1913), comb. nov., NHMUK PV R 3320, 4459 holotype. Skull in (A) dorsal, (B) ventral (palatal) and (C) right lateral views; partial mandible in 4460 (D) dorsal view. (E) Posterior section of the mandible in right lateral view; atlas in (F) anterior 4461 and (G) right lateral view. Refer to abbreviations list. Scale bars: $10 \mathrm{~cm}$ (A-D) and $2 \mathrm{~cm}(\mathrm{E}-\mathrm{G})$.

4462 Figure 17. Deslongchampsina larteti (Eudes-Deslongchamps, 1866a) Johnson, Young \&

4463 Brusatte, 2019, OUMNH J.29851, neotype. Skull in (A) dorsal, (B) ventral (palatal), (C) right 4464 lateral, (D) left lateral and (E) occipital views. Refer to abbreviations list. Scale bars: $5 \mathrm{~cm}$. 
4465 Figure 18. Proexochokefalos heberti (Morel de Glasville, 1876), comb. nov., MNHN.F 1890-13,

4466 holotype. Skull in (A) dorsal, (B) left lateral and (C) occipital views. Refer to abbreviations list.

4467 Scale bars: $10 \mathrm{~cm}$.

4468 Figure 19. Proexochokefalos cf. bouchardi (Sauvage, 1872), comb. nov. Unknown specimen

4469 number, photo provided by Y. Lepage (from Lepage et al., 2008). Skull in dorsal view. Refer to

4470 abbreviations list. Scale bar: $10 \mathrm{~cm}$.

4471 Figure 20. Steneosaurus rostromajor (Geoffroy Saint-Hilaire, 1825), MNHN.RJN 134c-d,

4472 nomen dubium. Partial rostrum in (A) dorsal, (B) ventral and (C) left lateral views. Refer to

4473 abbreviations list. Scale bar: $10 \mathrm{~cm}$.

4474 Figure 21. Andrianavoay baroni (Newton, 1893), comb. nov., NHMUK PV R 1999, holotype.

4475 Photograph of the partial skull and mandible in (A) right lateral view, as well as (B) partial

4476 rostrum in dorsal view; posterior skull in (C) dorsal and (D) ventral views; (E) partial mandible

4477 in dorsal view; and (F) fragment of osteoderm in dorsal view. Refer to abbreviations list. Scale

4478 bars: $10 \mathrm{~cm}(\mathrm{~A}), 5 \mathrm{~cm}(\mathrm{~B}-\mathrm{E})$ and $3 \mathrm{~cm} \mathrm{(F).}$

4479 Figure 22. Neosteneosaurus edwardsi (Eudes-Deslongchamps, 1868a), comb. nov. (A-C)

4480 MNHN.RJN 118, lectotype and (D-F) NHMUK PV R 2865, referred specimen. Partial skull in

4481 (A) dorsal, (B) ventral (palatal) and (C) right lateral views. Refer to abbreviations list. Scale

4482 bars: $10 \mathrm{~cm}$.

4483 Figure 23. Yvridiosuchus boutilieri (Eudes-Deslongchamps, 1868c) Johnson, Young \& Brusatte, 4484 2019. (A-D) OUMNH J.1401, holotype and (E-I) OUMNH J.29850, referred specimen. Skull in 
4485 (A, E) dorsal, (B, F) ventral (palatal), (C, G) right lateral, (D, H) left lateral and (I) occipital 4486 views. Refer to abbreviations list. Scale bars: $5 \mathrm{~cm}$.

4487 Figure 24. Lemmysuchus obtusidens (Andrews, 1909) Johnson et al., 2017, NHMUK PV R 3168, 4488 holotype. Skull in (A) dorsal, (B) occipital, (C) right lateral and (D) left lateral views. Refer to 4489 abbreviations list. Scale bars: $20 \mathrm{~cm}$.

4490 Figure 25. Machimosaurus buffetauti Young et al., 2015, SMNS 91415, holotype. Skull in (A) 4491 dorsal, (B) occipital, (C) right lateral and (D) left lateral views. Rostrum in (E) left lateral view, 4492 with a close-up of (i) the premaxillary alveoli. (F) Mandible in left lateral view. Refer to 4493 abbreviations list. Scale bars: $10 \mathrm{~cm}$.

4494 Figure 26. Machimosaurus mosae Sauvage \& Liénard, 1879, IRSNB cast. Not to scale.

4495 Figure 27. Machimosaurus hugii (von Meyer, 1837) emend. von Meyer, 1838, MG-8730, 4496 referred specimen. (A-C) MG-8730-2: occipital in (A) dorsal, (B) ventral and (C) occipital 4497 views. (D-E) MG-8730-1: partial rostrum in (D-E) palatal view. Refer to abbreviation list. Scale 4498 bars: $10 \mathrm{~cm}$.

4499 Figure 28. Machimosaurus rex Fanti et al., 2016, ONM NG 1-25, holotype. Partial skull in (A) 4500 ventral view, with a close-up of the (i) maxillary alveoli. Additional material: (B) dorsal vertebra 4501 in anterior view; (C) dorsal osteoderm; and (D) close-up of tooth apex. Refer to abbreviation list. 4502 Scale bars: $10 \mathrm{~cm}$ (as indicated on A), $5 \mathrm{~cm}(\mathrm{~B}-\mathrm{C})$ and $1 \mathrm{~cm}(\mathrm{D})$.

4503 Figure 29. Comparative photographs displaying ornamentation on the prefrontal (ch. 12), 4504 lacrimal (ch. 13) and frontal (ch. 15) in dorsal view. (A) Plagiophthalmosuchus gracilirostris 
4505 (NHMUK PV R 14892); (B) Clovesuurdameredeor stephani (NHMUK PV OR 49126); (C)

4506 Indosinosuchus potamosiamensis (PRC-11); (D) the Chinese teleosauroid (IVPP V 10098); (E)

4507 Mycterosuchus nasutus (NHMUK PV R 2617); (F) Charitomenosuchus leedsi (NHMUK PV R

4508 38060; (G) Neosteneosaurus edwardsi (NHMUK PV R 2865); (H) Yvridiosuchus boutilieri

4509 (OUMNH J.1401); and (I) Machimosaurus buffetauti (SMNS 91415). Scale bars: $4 \mathrm{~cm}$.

4510 Figure 30. Comparative photographs displaying premaxillary anteroposterior length relative to

4511 rostrum length (ch. 43): (A) Macrospondylus bollensis (SMNS 81672) and (B) the Chinese

4512 teleosauroid (IVPP V 10098), as well as (C) Metriorhynchus superciliosus (LPP.M.48). Dashed

4513 lines $(\cdots)$ represent anteroposterior premaxillary length, while solid lines $(-)$ represent total

4514 rostral length. Scale bars: $10 \mathrm{~cm}$.

4515 Figure 31. Comparative photographs displaying medial margins of the external nares (ch. 56)

4516 and the premaxilla-maxilla suture (ch. 58): (A) Mycterosuchus nasutus (CAMSM J.1420), (B)

4517 Bathysuchus megarhinus (unnumbered LPP specimen), (C) the Chinese teleosauroid (IVPP V

4518 10098), (D) Macrospondylus bollensis (MMG BwJ 565), (E) Deslongchampsina larteti

4519 (OUMNH J.29851), (F) Steneosaurus rostromajor (MNHN.RJN 134c-d), (G) Mystriosaurus

4520 laurillardi (NHMUK PV OR 14781), (H) Neosteneosaurus edwardsi (NHMUK PV R 2685) and

4521 (I) Charitomenosuchus leedsi (NHMUK PV R 3320). Scale bars: $3 \mathrm{~cm}$.

4522 Figure 32. Comparative photographs displaying the presence/absence of elongated posterior

4523 nasal processes (ch. 64), anteromedial frontal process (ch. 124) and additional anterolateral

4524 frontal projections (ch.125): (A) Indosinosuchus potamosiamensis (PRC-11), (B Mycterosuchus

4525 nasutus (NHMUK PV R 2617), (C) Macrospondylus bollensis (NHMW-1878-0047-0001), (D)

4526 Clovesuurdameredeor stephani (NHMUK PV OR 49126), (E) Charitomenosuchus leedsi 
4527 (NHMUK PV R 3320), Neosteneosaurus edwardsi ((F): MNHN.RJN 118; (G) NHMUK PV R

4528 2865), (H) Lemmysuchus obtusidens (LPP.M.21), (I) Machimosaurus buffetauti (SMNS91415)

4529 and (J) Platysuchus multiscrobiculatus (SMNS 9930). Platysuchus photograph provided by

4530 MTY. Scale bars: $4 \mathrm{~cm}$.

4531 Figure 33. Comparative photographs displaying the anterior elongation of the jugal (ch. 167) in

4532 (A) Plagiophthalmosuchus gracilirostris (NHMUK PV OR 14792); (B) Deslongchampsina

4533 larteti (OUMNH J.29851); (C) Charitomenosuchus leedsi (NHMUK PV R 3320); and (D)

4534 Proexochokefalos heberti (MNHN.F 1890-13). Scale bars: 5 cm.

4535 Figure 34. Comparative photographs displaying the premaxillary-maxillary suture in palatal view

4536 (ch. 184): (A) Teleosauroidea (Lemmysuchus obtusidens LPP.M.21) and (B) Metriorhynchoidea

4537 (Metriorhynchus supercilious LPP.M.48). Scale bars: $7 \mathrm{~cm}$.

4538 Figure 35. Comparative photographs displaying the exoccipital and paraoccipital processes (ch.

4539 208): (A) Plagiophthalmosuchus gracilirostris (MNHNL TU515), (B ‘Steneosaurus' sp.

4540 (IRSNB R 0140), (C) Proexochokefalos heberti (MNHN.F 1890-13), (D) Neosteneosaurus

4541 edwardsi (PETMG R178) and (E) Machimosaurus hugii (MG 8730). Scale bars: 5 cm.

4542 Figure 36. Comparative photographs displaying the Meckelian groove (canal) (ch. 269) in (A)

4543 Mycterosuchus nasutus (NHMUK PV R 2617), (B) Macrospondylus bollensis (53422), (C)

4544 Charitomenosuchus leedsi (NHMUK PV R 3806), (D) Steneosaurus hulkei (= Neosteneosaurus

4545 edwardsi) (NHMUK PV R 2074), (E) Yvridiosuchus boutilieri (OUMNH J.1404), (F)

4546 Lemmysuchus obtusidens (LPP.M.21), and (G) Machimosaurus mosae (Young et al., 2014).

4547 Scale bars: $3 \mathrm{~cm}$.

Peer) reviewing PDF | (2020:03:47227:2:0:NEW 29 Jul 2020) 
4548 Figure 37. Comparative photographs displaying the curvature of the retroarticular process (ch.

4549 270) (in lateral view). (A) Plagiophthalmosuchus gracilirostris (MNHNL TU515), (B)

4550 Mystriosaurus laurillardi (NHMUK PV OR 14781), (C) Mycterosuchus nasutus (NHMUK PV

4551 R 2617), (D) Charitomenosuchus leedsi (NHMUK PV R 3806), (E) Macrospondylus bollensis

4552 (SMNS 58876), (F) Proexochokefalos heberti (MNHN.F 1890-13), (G) Machimosaurus

4553 buffetauti (SMNS 91415) and (H) Yvridiosuchus boutilieri (OUMNH J.29850). Scale bars: 15

$4554 \mathrm{~cm}(\mathrm{~B}, \mathrm{E}-\mathrm{F})$ and $5 \mathrm{~cm}(\mathrm{~A}, \mathrm{C}-\mathrm{D}, \mathrm{G}-\mathrm{H})$.

4555 Figure 38. Comparative photographs displaying the reception pits (in right lateral view) (ch.

4556 291). (A) Plagiophthalmosuchus gracilirostris (NHMUK PV OR 15500), (B) Mystriosaurus

4557 laurillardi (NHMUK PV OR 14781), (C) Proexochokefalos heberti (MNHN.F 1890-13) and (D)

4558 Lemmysuchus obtusidens (LPP.M.21). Scale bars: $17 \mathrm{~cm}$.

4559 Figure 39. Comparative photographs displaying characteristic features of the premaxillary 4560 alveoli (ch. 292 to 297), in: (A) the Chinese teleosauroid (IVPP V 10098), (B) Bathysuchus 4561 megarhinus (DORCM G.05067i; Foffa et al., 2019), (C) Indosinosuchus potamosiamensis (PRC-

4562 11), (D) Platysuchus multiscrobiculatus (MNHNL. TU895), (E) Charitomenosuchus leedsi

4563 (NHMUK PV R 3806), (F) Mystriosaurus sp. (SNHM-IG-008-R), (G) Yvridiosuchus boutilieri

4564 (OUMNH J.1401) and (H) Lemmysuchus obtusidens (LPP.M.21). Note that character 294 and 4565295 are inapplicable for the Chinese teleosauroid (IVPP V 10098). Scale bars: $3 \mathrm{~cm}$.

4566 Figure 40. Comparative photographs of teleosauroid teeth, highlighting the carinae (ch. 3394567 340), apices (ch. 327) and anastomosing pattern (ch. 358): (A) Bathysuchus megarhinus 4568 (DORCM G.05067iv; Foffa et al., 2019), (B) Sericodon jugleri (NRM-PZ R.2337), (C) 4569 Proexochokefalos heberti (MNHN.F 1890-13), (D) Deslongchampsina larteti (OUMNH 
4570 J.29851), (F) Neosteneosaurus edwardsi (NHMUK PV R 2865), (F) Machimosaurini

4571 indeterminate (GPIT-RE-301), (G) Yvridiosuchus boutilieri (OUMNH J.29850), and (H)

4572 Machimosaurus hugii (MG 25). Scale bars: $3 \mathrm{~cm}(\mathrm{~A}-\mathrm{B}, \mathrm{E})$ and $1 \mathrm{~cm}(\mathrm{C}-\mathrm{D}, \mathrm{F}-\mathrm{H})$.

4573 Figure 41. Comparative photographs of teleosauroid cervical ribs (ch. 394): (A) Macrospondylus

4574 bollensis (SMNS 51984), (B) Mycterosuchus nasutus (NHMUK PV R 2617), (C)

4575 Neosteneosaurus edwardsi (NHMUK PV R 3701) and (D) Lemmysuchus obtusidens (NHMUK

4576 PV R 3168). Scale bars: $3 \mathrm{~cm}$.

4577 Figure 42. Comparative photographs of teleosauroid dorsal ribs (ch. 395 and 396) (from the

4578 middle of the ribcage); (A) Charitomenosuchus leedsi (NHMUK PV R 3806), (B)

4579 Neosteneosaurus edwardsi (PETMG R178), (C) Lemmysuchus obtusidens (NHMUK PV R

4580 3168) and (D) Macrospondylus bollensis (SMNS 52034). Scale bars: $3 \mathrm{~cm}$.

4581 Figure 43. Comparative photographs of teleosauroid sacral vertebrae, with special attention to 4582 the number (ch. 379) and flange of the second sacral (ch. 398): (A) Charitomenosuchus leedsi 4583 (NHMUK PV R 3806), (B) Lemmysuchus obtusidens (NHMUK PV R 3168), (C) Mycterosuchus 4584 nasutus (NHMUK PV R 2617) and (D) Macrospondylus bollensis (GPIT-RE-9427).

4585 Figure 44. Comparative photographs of teleosauroid ulnae and radiae, with special attention to 4586 relative size (ch. 417) and proximal ulna (ch. 420): (A) Neosteneosaurus edwardsi (PETMG 4587 R178) i. ulna and ii. radius; (B) Mycterosuchus nasutus (NHMUK PV R 2617) i. ulna and ii. 4588 radius; (C) Charitomenosuchus leedsi (NHMUK PV R 3806) i. ulna and ii. radius; and (D) 4589 Macrospondylus bollensis (SMNS 53422) i. ulna and ii. radius. Scale bars: $3 \mathrm{~cm}$. 
4590 Figure 45. Comparative photographs of teleosauroid pubes, highlighting the pubic blade (ch.

4591 430) and elongation (ch. 431): (A) Mycterosuchus nasutus (NHMUK PV R 2617), (B)

4592 Charitomenosuchus leedsi (NHMUK PV R 3806), (C) Neosteneosaurus edwardsi (PETMG

4593 R178) and (D) Macrospondylus bollensis (SMNS 51957). Scale bars: $3 \mathrm{~cm}$.

4594 Figure 46. Comparative photographs of teleosauroid ilia with attention to the anterior process

4595 (ch. 434), supraacetabular crest (ch. 438) and postacetabular process (ch. 440): (A)

4596 Charitomenosuchus leedsi (NHMUK PV R 3806), (B) Macrospondylus bollensis (SMNS

4597 18672), (C) Neosteneosaurus edwardsi (PETMG R178) and (D) Lemmysuchus obtusidens

4598 (NHMUK PV R 3168). Scale bars: $5 \mathrm{~cm}$.

4599 Figure 47. Comparative photographs of teleosauroid ischia with emphasis on the ischial blade

4600 (ch. 449): (A) Platysuchus multiscrobiculatus (SMNS 9930), (B) Teleosaurus sp. (NHMUK PV

4601 238), (C) Neosteneosaurus edwardsi (NHMUK PV R 3898), (D) Macrospondylus bollensis

4602 (SMNS 58876), (E) Aeolodon priscus (MNHN.F.CNJ 78), (F) Lemmysuchus obtusidens

4603 (NHMUK PV R 3168) and (G) Machimosaurus mosae (IRSNB cast). Scale bars: $3 \mathrm{~cm},(\mathrm{H})$ not 4604 to scale.

4605 Figure 48. Comparative photographs of teleosauroid femora (ch. 456 and 459): Mycterosuchus 4606 nasutus (NHMUK PV R 2617) [(A) femoral head dorsal view; (B) femoral condyles posterior 4607 view], Neosteneosaurus edwardsi (PETMG R178) [(C) femoral head dorsal view; (D) femoral 4608 condyles posterior view] and Macrospondylus bollensis (SMNS 51555) ((E) femoral condyles 4609 posterior view). Scale bars: $3 \mathrm{~cm}$, (E) not to scale. 
4610 Figure 49. Comparative photographs of teleosauroid tibiae, focusing on the tibal tuberosity (ch.

4611 464): (A) Charitomenosuchus leedsi (NHMUK PV R 3806), (B) Neosteneosaurus edwardsi

4612 (PETMG R178) and (C) Lemmysuchus obtusidens (NHMUK PV R 3168). Scale bars: $3 \mathrm{~cm}$.

4613 Figure 50. Comparative photographs of teleosauroid calcaneae and astragulae (ch. 466):

4614 Mycterosuchus nasutus (NHMUK PV R 2617) [(A-B) calcaneum in (A) dorsal and (B) lateral

4615 view; and (C) astragulus)], (D) Macrospondylus bollensis (SMNS 81699) and (E)

4616 Neosteneosaurus edwardsi (PETMG R175). Scale bars: $1.5 \mathrm{~cm}$ (A-C) and $2.5 \mathrm{~cm}$ (D), (E) not to 4617 scale.

4618 Figure 51. Comparative photographs displaying teleosauroid dorsal sacral osteoderms, with 4619 emphasis on ornamentation pattern (ch. 473) and keel presence (ch. 489): (A)

4620 Plagiophthalmosuchus gracilirostris (NHMUK PV OR 14892), (B) Charitomenosuchus leedsi

4621 (NHMUK PV R 3806), (C) Teleosaurus cadomensis (NHMUK PV R 119a), (D) Mycterosuchus

4622 nasutus (NHMUK PV R 2617), (E) Neosteneosaurus edwardsi (PETMG R178), and (F)

4623 Lemmysuchus obtusidens (NHMUK PV R 3168). Scale bars: $3 \mathrm{~cm}$, (D) not to scale.

4624 Figure 52. Comparative photographs displaying telescopic orbits (ch. 10, 151) as well as 4625 presence or absence (ch. 83) and shape of antorbital fenestrae (ch. 86) in dorsal view. (A) 4626 Plagiophthalmosuchus gracilirostris (NHMUK PV OR 14892); (B) Deslongchampsina larteti

4627 (OUMNH J.29851); (C) Indosinosuchus potamosiamensis (PRC-11); (D) Mycterosuchus 4628 nasutus (NHMUK PV R 2617); (E) Charitomenosuchus leedsi (NHMUK PV R 3806); (F) 4629 Yvridiosuchus boutilieri (OUMNH J.1401); (G) Proexochokefalos heberti (MNHN.F 1890-13); 4630 (H) Neosteneosaurus edwardsi (PETMG R178); and (I) Lemmysuchus obtusidens (LPP.M.21). 
4631 Note the shallow antorbital fenestrae of $C$. leedsi compared to other taxa with antorbital

4632 fenestrae. Scale bars: $4 \mathrm{~cm}$.

4633 Figure 53. Comparative photographs displaying the anterior and anterolateral premaxillary

4634 margins (ch. 48) as well as neurovascular foramina (ch. 27), in lateral view: (A) Macrospondylus

4635 bollensis (SMNS 51563), (B) Mystriosaurus laurillardi (NHMUK PV OR 14781), (C)

4636 Machimosaurus buffetauti (SMNS 91415), (D) Lemmysuchus obtusidens (LPP.M.21), (E)

4637 Yvridiosuchus boutilieri (OUMNH J.1401) and (F) Indosinosuchus kalasinensis (PRC-239).

4638 Scale bars: $5 \mathrm{~cm}$.

4639 Figure 54. Comparative photographs displaying the external nares, in dorsal view (ch. 34): (A)

4640 the Chinese teleosauroid (IVPP V 10098), (B) Mystriosaurus laurillardi (HLMD V946-948), (C)

4641 Bathysuchus megarhinus (unnumbered LPP specimen), (D) Deslongchampsina larteti (OUMNH

4642 J.29851), (E) Neosteneosaurus edwardsi (NHMUK PV R 3701) and (F) Lemmysuchus

4643 obtusidens (LPP.M.21). Scale bars $3 \mathrm{~cm}$.

4644 Figure 55. Comparative photographs displaying the shape of the supratemporal fenestrae (ch.

4645 102), as well as the anterolateral expansion of the anterior portion (ch. 103) and elongation (ch.

4646 104) of these fenestrae in dorsal view. (A) Teleosaurus cadomensis (MNHN AC 8746; (B)

4647 Mystriosaurus laurillardi (NHMUK PV OR 14781); (C) Plagiophthalmosuchus gracilirostris

4648 (NHMUK PV OR 14892); (D) Macrospondylus bollensis (MMG BwJ 565); (E)

4649 Clovesuurdameredeor stephani (NHMUK PV OR 49126), (F) Proexochokefalos heberti

4650 (MNHN.F 1890-13); and (G) Lemmysuchus obtusidens (NHMUK PV R 3168). Scale bars: $3 \mathrm{~cm}$

4651 (A, C) and $10 \mathrm{~cm}(\mathrm{~B}, \mathrm{D}-\mathrm{F})$. 
4652 Figure 56. Comparative photographs of teleosauroid orbital margin (in lateral view), focusing on

4653 the inclusion of the postorbital (ch. 158): (A) Plagiophthalmosuchus gracilirostris (NHMUK PV

4654 OR 14892), (B) Clovesuurdameredeor stephani (NHMUK PV OR 49126), (C) the Chinese

4655 teleosauroid (IVPP V 10098) and (D) Teleosaurus cadomensis (MNHN AC 8746). Scale bars: 3

$4656 \mathrm{~cm}$.

4657 Figure 57. Comparative photographs exhibiting exposure of the teleosauroid basioccipital (ch.

4658 225): (A) Mycterosuchus nasutus (CAMSM J.1420), (B) the Chinese teleosauroid (IVPP V

4659 10098), (C) Charitomenosuchus leedsi (NHMUK PV R 3320) and (D) Neosteneosaurus

4660 edwardsi (NHMUK PV R 2865). Scale bars: $7 \mathrm{~cm}$.

4661 Figure 58. Comparative photographs of teleosauroid humeri (ch. 410): (A) Mycterosuchus

4662 nasutus (NHMUK PV R 2617), (B) Macrospondylus bollensis (SMNS 18672), (C)

4663 Neosteneosaurus edwardsi (NHMUK PV R 3701), (D) Charitomenosuchus leedsi (NHMUK PV

4664 R 3806) and (E) Aeolodon priscus (MNHN.F.CNJ 78). Scale bars: $3 \mathrm{~cm}$.

4665 Figure 59. Results of the unweighted parsimonious phylogenetic analysis, focusing on

4666 Teleosauroidea. (A) simplified strict consensus topology (125 MPTs and 1659 steps: CI $=0.405$,

$4667 \mathrm{RI}=0.844$ ); (B) simplified strict consensus topology excluding $S$. rostromajor (176 MPTs and

46681659 steps: $\mathrm{CI}=0.405, \mathrm{RI}=0.844)$; and $(\mathrm{C})$ parsimonious majority rules topology $(160 \mathrm{MPTs}$

4669 and 1619 steps). In all topologies Teleosauroidea is monophyletic and two distinct families (T

4670 and M) are recovered. Bremer support and jackknife values (Bremer/jackknife; A-B) and support

4671 percentages (C) are included. 
4672 Figure 60. Results of the extended weighted parsimonious phylogenetic analysis, focusing on

4673 Teleosauroidea. (A) Simplified strict consensus topology with extended implied weighting

$4674 \quad(\mathrm{k}=12)$ of the 47 MPTs; (B) simplified strict consensus topology with extended implied

4675 weighting $(\mathrm{k}=12)$ excluding $S$. rostromajor (39 MPTs); and (C) agreement subtree (based on the

4676 unweighted strict consensus) of Teleosauroidea.

4677 Figure 61. Simplified consensus topology, produced in MrBayes using gamma distribution

4678 (rates $=$ gamma), standard deviation $=0.019863$, harmonic mean $=-7785.47$. Note that $S$.

4679 rostromajor is recovered as most closely related to Machimosaurini.

4680 Figure 62. Photographs of three well preserved taxa not included in our dataset: (A)

4681 Steneosaurus pictaviensis (= Charitomenosuchus leedsi) LPP.M.37; (B) Steneosaurus depressus

4682 (= Proexochokefalos heberti) OUMNH J.01420; and (C) Steneosaurus hulkei (=

4683 Neosteneosaurus edwardsi) (NHMUK PV R 2074). See text for in-depth explanation as to why

4684 these taxa are excluded. Scale bars: $4 \mathrm{~cm}(\mathrm{~A}, \mathrm{C})$ and $10 \mathrm{~cm}(\mathrm{~B})$.

4685 Figure 63. Hypothesized teleosauroid ecomorphologies mapped onto the extended implied

4686 weighted topology (excluding Steneosaurus rostromajor: 77 MPTs): (A) rostral morphology; (B)

4687 feeding ecology; and (C) palaeohabitat. Note that Family T is more phenotypically plastic than

4688 Family $\mathrm{M}$ in terms of (A) rostrum and (C) habitat, and that Family $\mathrm{M}$ shows a distinctive, linear

4689 shift in (A) rostral length and (B) feeding style.

4690 Figure 64. Summary of time-calibrated phylogeny (extended implied weighting excluding

4691 Steneosaurus rostromajor: 39 MPTs) of teleosauroids, focusing on number $\left(\mathrm{n}^{\circ}\right)$ of

4692 ecomorphological guilds present during four main time periods (Toarcian, Bathonian, Callovian 
4693 and Kimmeridgian). Major guilds are as follows: dark blue = longirostrine specialist; purple $=$

4694 mesorostrine generalist; light blue = pelagic generalist; black = longirostrine generalist; yellow = 4695 macrophagous/durophagous; red = semi-terrestrial generalist. Grey coloured lines indicate 4696 unknown ecomorphology, due to incomplete material. Note that the number of guilds remains 4697 constant (four) until the Kimmeridgian, in which there is a drop (three). Silhouettes provided by 4698 PhyloPic (G. Monger, S. Hartman and N. Tamara). 


\section{Table 1 (on next page)}

Teleosauroid ecomorphotypes by species in four time periods.

Table 1. List of teleosauroid ecomorphotypes in four main time periods: the Toarcian, Bathonian, Callovian and Kimmeridgian. Note that S. rostromajor (Oxfordian), Indosinosuchus (Late Jurassic) and Mac. rex (Hauterivian-Barremian) are not included. 
1 Table 1. List of teleosauroid ecomorphotypes in four main time periods: the Toarcian, Bathonian,

2 Callovian and Kimmeridgian. Note that S. rostromajor (Oxfordian), Indosinosuchus (Late

3 Jurassic) and Mac. rex (Hauterivian-Barremian) are not included.

\begin{tabular}{|c|c|c|c|}
\hline TAXA & PERIOD & ECOMORPH & $\begin{array}{l}\text { CHARACTERISTIC } \\
\text { FEATURES }\end{array}$ \\
\hline $\begin{array}{l}\text { Plagiophthalmosuchus } \\
\text { gracilirostris }\end{array}$ & Toarcian & $\begin{array}{l}\text { Longirostrine, } \\
\text { specialist }\end{array}$ & $\begin{array}{l}\text { Lateral orbits; elongated } \\
\text { snout; slender pointed } \\
\text { teeth }\end{array}$ \\
\hline $\begin{array}{l}\text { Macrospondylus } \\
\text { bollensis }\end{array}$ & Toarcian & $\begin{array}{l}\text { Longirostrine, } \\
\text { generalist }\end{array}$ & $\begin{array}{l}\text { Dorsal orbits; dorsal } \\
\text { nares; 'generalized body } \\
\text { plan }\end{array}$ \\
\hline $\begin{array}{l}\text { Platysuchus } \\
\text { multiscrobiculatus }\end{array}$ & Toarcian & $\begin{array}{l}\text { Longirostrine, } \\
\text { semi- } \\
\text { terrestrial }\end{array}$ & $\begin{array}{l}\text { Dorsal orbits; anterior } \\
\text { nares; heavy, integrated } \\
\text { dorsal shield }\end{array}$ \\
\hline $\begin{array}{l}\text { Mystriosaurus } \\
\text { laurillardi, Chinese } \\
\text { teleosauroid }\end{array}$ & Toarcian & $\begin{array}{l}\text { Mesorostrine, } \\
\text { generalist }\end{array}$ & $\begin{array}{l}\text { Dorsal orbits; anterior } \\
\text { nares; shorter rostrum }\end{array}$ \\
\hline $\begin{array}{l}\text { Seldsienean } \\
\text { megistorhynchus }\end{array}$ & Bathonian & $\begin{array}{l}\text { Longirostrine, } \\
\text { generalist }\end{array}$ & $\begin{array}{l}\text { Dorsal orbits and nares; } \\
\text { slender, elongated jaws }\end{array}$ \\
\hline $\begin{array}{l}\text { Deslongchampsina } \\
\text { larteti }\end{array}$ & Bathonian & $\begin{array}{l}\text { Mesorostrine, } \\
\text { generalist }\end{array}$ & $\begin{array}{l}\text { Dorsal orbits and nares; } \\
\text { robust pointed teeth }\end{array}$ \\
\hline $\begin{array}{l}\text { Teleosaurus } \\
\text { cadomensis }\end{array}$ & Bathonian & $\begin{array}{l}\text { Longirostrine, } \\
\text { semi- } \\
\text { terrestrial }\end{array}$ & $\begin{array}{l}\text { Dorsal orbits; 'spindly' } \\
\text { teeth; heavy, integrated } \\
\text { dorsal shield }\end{array}$ \\
\hline $\begin{array}{l}\text { Yvridiosuchus } \\
\text { boutilieri }\end{array}$ & Bathonian & $\begin{array}{l}\text { Mesorostrine, } \\
\text { durophagous }\end{array}$ & $\begin{array}{l}\text { Enlarged fenestrae; } \\
\text { anastomosed blunt teeth }\end{array}$ \\
\hline $\begin{array}{l}\text { Andrianavoay baroni, } \\
\text { Clovesuurdameredeor } \\
\text { stephani }\end{array}$ & Bathonian & Unknown & $\mathrm{N} / \mathrm{A}$ \\
\hline $\begin{array}{l}\text { Charitomenosuchus } \\
\text { leedsi }\end{array}$ & Callovian & $\begin{array}{l}\text { Longirostrine, } \\
\text { generalist }\end{array}$ & $\begin{array}{l}\text { Dorsal orbits and nares; } \\
\text { gracile skeleton }\end{array}$ \\
\hline $\begin{array}{l}\text { Mycterosuchus } \\
\text { nasutus }\end{array}$ & Callovian & $\begin{array}{l}\text { Longirostrine, } \\
\text { semi- } \\
\text { terrestrial }\end{array}$ & $\begin{array}{l}\text { Dorsal orbits and nares; } \\
\text { protruding orbits; } \\
\text { relatively elongated } \\
\text { limbs; heavy osteoderms }\end{array}$ \\
\hline $\begin{array}{l}\text { Neosteneosaurus } \\
\text { edwardsi, } \\
\text { Proexochokefalos } \\
\text { heberti }\end{array}$ & Callovian & $\begin{array}{l}\text { Mesorostrine, } \\
\text { generalist }\end{array}$ & $\begin{array}{l}\text { Dorsal orbits and nares; } \\
\text { robust, elongated skulls; } \\
\text { large teeth }\end{array}$ \\
\hline $\begin{array}{l}\text { Lemmysuchus } \\
\text { obtusidens }\end{array}$ & Callovian & $\begin{array}{l}\text { Mesorostrine, } \\
\text { durophagous }\end{array}$ & $\begin{array}{l}\text { Enlarged fenestrae; } \\
\text { anastomosed blunt teeth; } \\
\text { reduced postcrania }\end{array}$ \\
\hline
\end{tabular}




\begin{tabular}{|l|l|l|l|}
\hline $\begin{array}{l}\text { Proexochokefalos } \text { cf. } \\
\text { bouchardi }\end{array}$ & Kimmeridgian & $\begin{array}{l}\text { Mesorostrine, } \\
\text { generalist }\end{array}$ & $\begin{array}{l}\text { Dorsal orbits and nares; } \\
\text { robust elongated skull }\end{array}$ \\
\hline $\begin{array}{l}\text { Machimosaurus } \\
\text { buffetauti, } \\
\text { Machimosaurus } \\
\text { mosae, } \\
\text { Machimosaurus hugii }\end{array}$ & Kimmeridgian & $\begin{array}{l}\text { Mesorostrine, } \\
\text { durophagous }\end{array}$ & $\begin{array}{l}\text { Enlarged fenestrae; } \\
\text { anastomosed blunt teeth; } \\
\text { reduced postcrania }\end{array}$ \\
\hline $\begin{array}{l}\text { Sericodon jugleri, } \\
\text { Bathysuchus } \\
\text { megarhinus, Aeolodon } \\
\text { priscus }\end{array}$ & Kimmeridgian & $\begin{array}{l}\text { Longirostrine, } \\
\text { pelagic }\end{array}$ & $\begin{array}{l}\text { Protruding orbits; weakly } \\
\text { ornamented skull; } \\
\text { reduced osteoderms; } \\
\text { forelimb reduced }\end{array}$ \\
\hline
\end{tabular}

4 


\section{Table 2 (on next page)}

Teleosauroid localities and material by genera.

Table 2. Comprehensive list of localities where teleosauroid material has been found;

grouped by genera and includes 'Steneosaurus', 'Teleosaurus' and Machimosaurus spp. 
1 Table 2. Comprehensive list of localities where teleosauroid material has been found; grouped by

2 genera and includes 'Steneosaurus', 'Teleosaurus' and Machimosaurus spp.

\begin{tabular}{|c|c|c|c|}
\hline GENERA & COUNTRY & LOCALITY & $\begin{array}{l}\text { MATERIAL } \\
\text { FOUND }\end{array}$ \\
\hline Plagiophthalmosuchus & $\begin{array}{l}\text { Luxembourg; } \\
\text { UK }\end{array}$ & Dudelange; Whitby & $\begin{array}{l}\text { Partial skeleton; } \\
\text { skull }\end{array}$ \\
\hline Platysuchus & $\begin{array}{l}\text { Germany; } \\
\text { Luxembourg }\end{array}$ & Holzmaden; Foetz & $\begin{array}{l}\text { Complete skeleton; } \\
\text { rostrum }\end{array}$ \\
\hline Mystriosaurus & Germany; UK & Altdorf; Whitby & $\begin{array}{l}\text { Complete and } \\
\text { partial skulls }\end{array}$ \\
\hline Chinese teleosauroid & China & Daxian & Complete skull \\
\hline Macrospondylus & $\begin{array}{l}\text { Germany; } \\
\text { ?France; } \\
\text { Luxembourg; } \\
\text { UK }\end{array}$ & $\begin{array}{l}\text { Holzmaden; Bad Boll; Ohmden; } \\
\text { Altdorf; Banz; Berg; } \\
\text { Schlierbach; Ohmenhausen; } \\
\text { ?Yonne; Sanem; Whitby; } \\
\text { Sandsend; Greens-Norton }\end{array}$ & $\begin{array}{l}\text { Multiple complete } \\
\text { and partial } \\
\text { specimens, as well } \\
\text { as cranial and } \\
\text { postcranial } \\
\text { material }\end{array}$ \\
\hline Deslongchampsina & France; UK & Calvados; Enslow Bridge & $\begin{array}{l}\text { Near complete } \\
\text { skulls }\end{array}$ \\
\hline Clovesuurdameredeor & UK & Closworth & $\begin{array}{l}\text { Partial skull and } \\
\text { mandible }\end{array}$ \\
\hline Yvridiosuchus & France; UK & Calvados; Enslow Bridge & $\begin{array}{l}\text { Complete and } \\
\text { partial skulls and } \\
\text { mandibles }\end{array}$ \\
\hline Teleosaurus & ?China; France & $\begin{array}{l}\text { ?Sichuan province; Allemagne; } \\
\text { Calvados }\end{array}$ & $\begin{array}{l}\text { Partial cranium; } \\
\text { osteoderms; } \\
\text { postcranial } \\
\text { material }\end{array}$ \\
\hline Andrianavoay & Madagascar & Unknown & $\begin{array}{l}\text { Partial skull and } \\
\text { mandible, } \\
\text { osteoderm } \\
\text { fragment }\end{array}$ \\
\hline Seldsienean & UK & Enslow Bridge; Kirtlington & Partial mandibles \\
\hline
\end{tabular}




\begin{tabular}{|c|c|c|c|}
\hline Lemmysuchus & France; UK & Unknown; Peterborough & $\begin{array}{l}\text { Complete skull and } \\
\text { partial mandible; } \\
\text { near complete } \\
\text { skeleton and } \\
\text { additional skull } \\
\text { material }\end{array}$ \\
\hline Charitomenosuchus & UK & Peterborough & $\begin{array}{l}\text { Near compete } \\
\text { skeleton as well as } \\
\text { additional skull } \\
\text { and postcranial } \\
\text { material }\end{array}$ \\
\hline Mycterosuchus & Germany; UK & Unknown; Peterborough & $\begin{array}{l}\text { Complete skulls } \\
\text { and postcranial } \\
\text { material }\end{array}$ \\
\hline Neosteneosaurus & UK & Peterborough & $\begin{array}{l}\text { Near compete } \\
\text { skeleton as well as } \\
\text { additional skull } \\
\text { and postcranial } \\
\text { material }\end{array}$ \\
\hline Proexochokefalos & $\begin{array}{l}\text { France; } \\
\text { Switzerland }\end{array}$ & $\begin{array}{l}\text { Villers-sur-Mer; Villerville; } \\
\text { Courtedoux-sur Combe Ronde }\end{array}$ & $\begin{array}{l}\text { Complete and } \\
\text { partial skulls; few } \\
\text { postcranial } \\
\text { material }\end{array}$ \\
\hline Machimosaurus & $\begin{array}{l}\text { Ethiopia; } \\
\text { France; } \\
\text { Germany; } \\
\text { Portugal; } \\
\text { Spain; } \\
\text { Switzerland; } \\
\text { Tunisia }\end{array}$ & $\begin{array}{l}\text { Feyambiro; Ain; Ambleteuse; } \\
\text { Cricqueboeuf; Issoncourt; } \\
\text { Neuffen; Leiria; Lagares; } \\
\text { Lourinhã; Malhão-Algarve; } \\
\text { Peralta; Porto das Barcas; } \\
\text { Zimbral; Asturias; Buñol; } \\
\text { Solothurn; Touil el Mhahir }\end{array}$ & $\begin{array}{l}\text { Skulls, mandibles, } \\
\text { postcrania; } \\
\text { numerous isolated } \\
\text { teeth }\end{array}$ \\
\hline Indosinosuchus & Thailand & Pho Noi & $\begin{array}{l}\text { Multiple skulls and } \\
\text { partial postcranial } \\
\text { material }\end{array}$ \\
\hline Sericodon & $\begin{array}{l}\text { Germany; } \\
\text { Switzerland }\end{array}$ & $\begin{array}{l}\text { Hannover; Ahlem; Tönniesberg; } \\
\text { Courtedoux-Bois de Sylleux; } \\
\text { Courtedoux-sur Combe Ronde; } \\
\text { Courtedoux-Tchâfouè; } \\
\text { Courtedoux-Vâ Tche Tchâ }\end{array}$ & $\begin{array}{l}\text { Numerous teeth; } \\
\text { partial skull and } \\
\text { postcranial } \\
\text { material }\end{array}$ \\
\hline
\end{tabular}




\begin{tabular}{|c|c|c|c|}
\hline Aeolodon & $\begin{array}{l}\text { France; } \\
\text { Germany }\end{array}$ & Canjuers; Daiting & $\begin{array}{l}\text { Near complete } \\
\text { skeletons }\end{array}$ \\
\hline Bathysuchus & France; UK & Quercy; Kimmeridge & $\begin{array}{l}\text { Nearly complete } \\
\text { skull and partial } \\
\text { mandible; rostral } \\
\text { material }\end{array}$ \\
\hline 'Steneosaurus' sp. & $\begin{array}{l}\text { Belgium; } \\
\text { France; } \\
\text { Germany; } \\
\text { India; Poland; } \\
\text { Russia; UK }\end{array}$ & $\begin{array}{l}\text { Lorraine; Poitiers; Vaches } \\
\text { Noires; Bartenbach; Bhuj; } \\
\text { Czarnogłowy; Dagestan; } \\
\text { Kirtlington; Whittlesea }\end{array}$ & $\begin{array}{l}\text { Partial rostra and } \\
\text { skulls; postcranial } \\
\text { material; teeth }\end{array}$ \\
\hline 'Teleosaurus'sp. & $\begin{array}{l}\text { China; India; } \\
\text { UK }\end{array}$ & $\begin{array}{l}\text { Beipei; Kota; Kirtlington; Slape } \\
\text { Hill Quarry }\end{array}$ & $\begin{array}{l}\text { Postcranial } \\
\text { material; } \\
\text { osteoderms }\end{array}$ \\
\hline Machimosaurus sp. & $\begin{array}{l}\text { France; } \\
\text { Portugal; } \\
\text { Spain; } \\
\text { Switzerland; } \\
\text { UK }\end{array}$ & $\begin{array}{l}\text { Haudainville; Porto das Barcas; } \\
\text { Peralta; Zimbral; Buñol; } \\
\text { Moutier; Oker quarry; Solothurn; } \\
\text { Lyme Regis; Dorset }\end{array}$ & Teeth \\
\hline
\end{tabular}

3

4 
Figure 1

Previous thalattosuchian topologies.

Figure 1. Recent strict consensus topologies focused on thalattosuchian phylogenetics, with attention to teleosauroids. Altered from (A) Mueller-Töwe (2006); (B) Jouve (2009); (C) Wilberg (2015b); (D) Johnson et al. (2019); and (E) Martin et al. (2019).
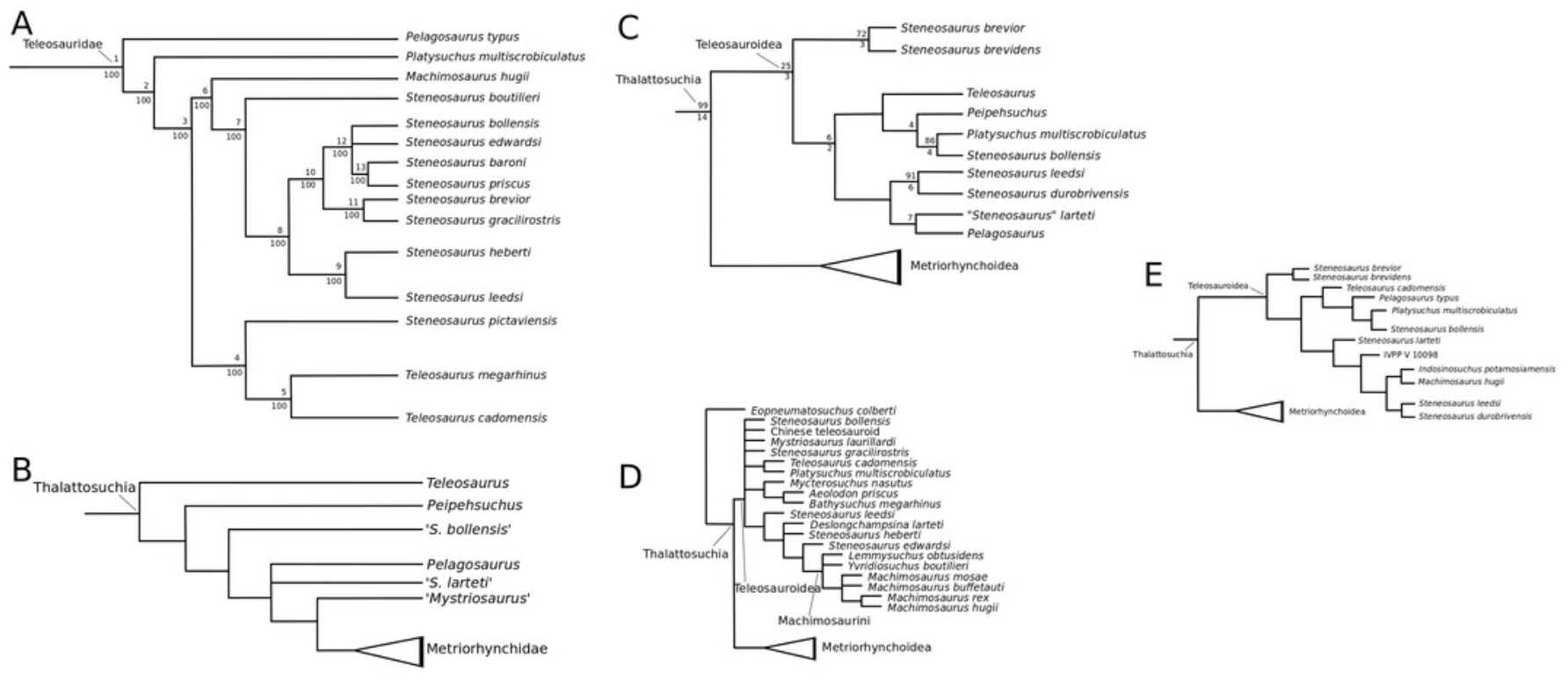


\section{Figure 2}

Plagiophthalmosuchus gracilirostris.

Figure 2. Plagiophthalmosuchus gracilirostris (Westphal, 1961) comb. nov., NHMUK PV OR 14792, holotype. (A) Nearly complete skeleton, with close-up views of: (B) the skull, (B) forelimb and (D) pelvic area. Refer to abbreviations list. Scale bars: $10 \mathrm{~cm}$ (A-B) and $4 \mathrm{~cm}$ (CD).

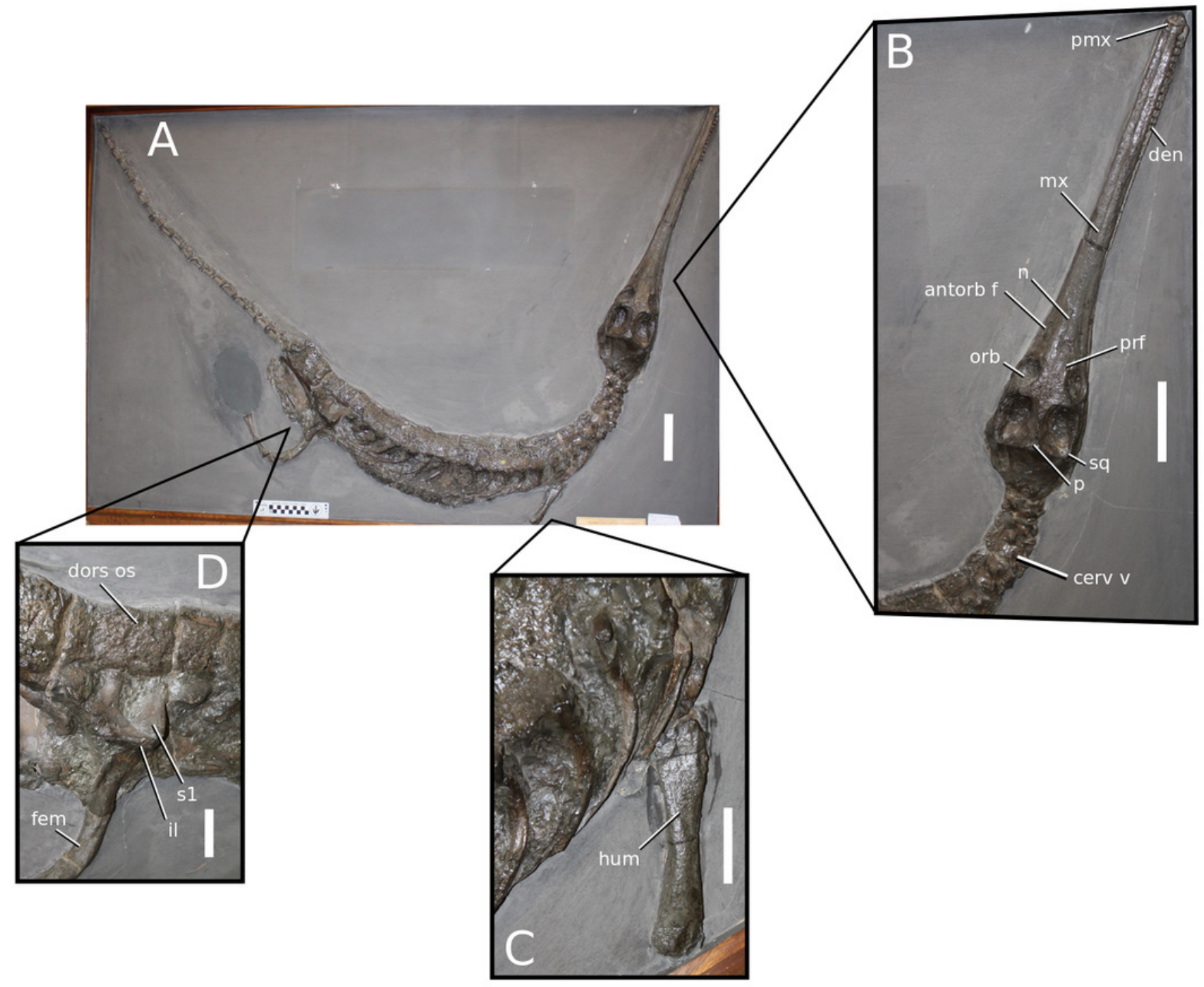




\section{Figure 3}

Mystriosaurus laurillardi.

Figure 3. Mystriosaurus laurillardi Kaup, 1834, holotype HLMD V946-948 (A-C) and referred specimen NHMUK PV OR 14781 (D-F). (A, D) Dorsal, (B) left lateral, (C, F) ventral and (E) right lateral views. Refer to abbreviations list. Scale bars: $10 \mathrm{~cm}$. Photographs $A$ to $C$ provided by S. Sachs.
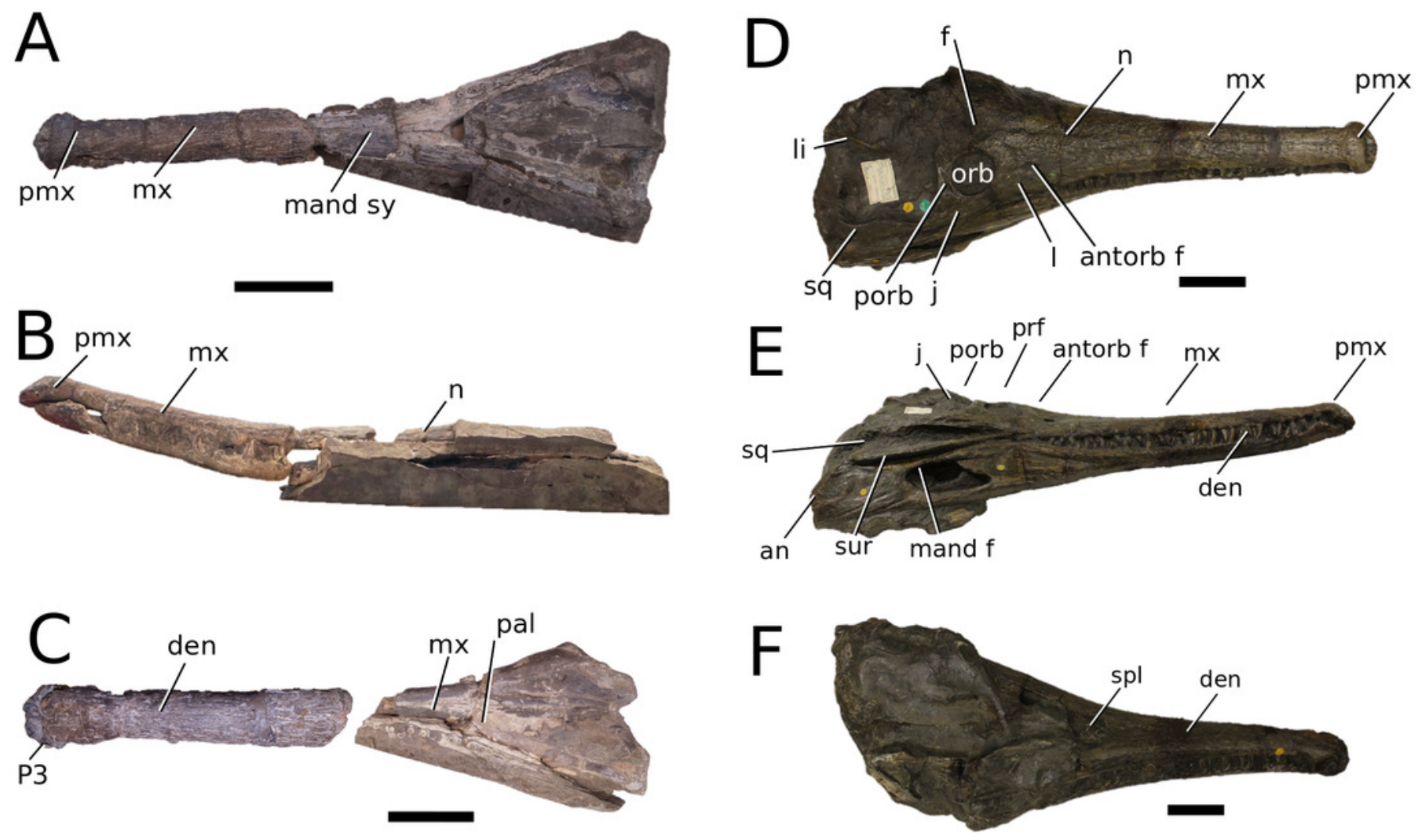
Figure 4

Clovesuurdameredeor stephani.

Figure 4. Clovesuurdameredeor stephani (Hulke, 1877), comb. nov., NHMUK PV OR 49126, holotype. Skull in (A) dorsal, (B) ventral (palatal), (C) right and (D) left lateral views. Partial mandible in (E) dorsal view, and right retroarticular process in (F) dorsal and (G) right lateral views. Refer to abbreviations list. Scale bars: $10 \mathrm{~cm}(\mathrm{~A}-\mathrm{C})$ and $4 \mathrm{~cm}(\mathrm{E}-\mathrm{F})$.
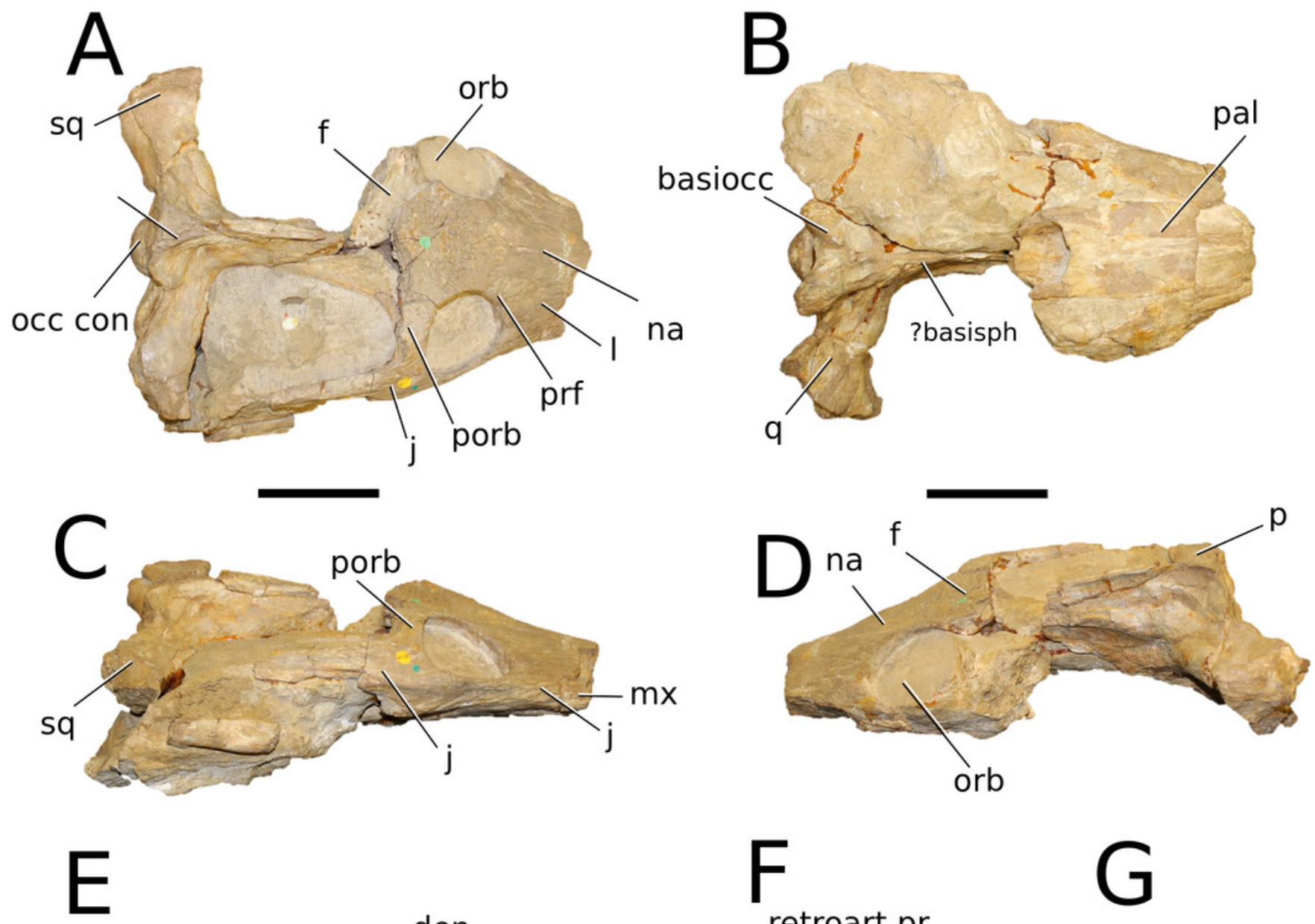

F
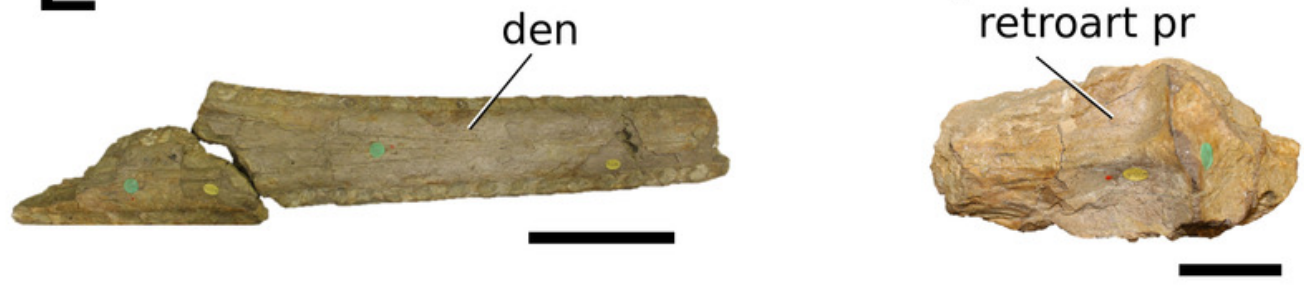
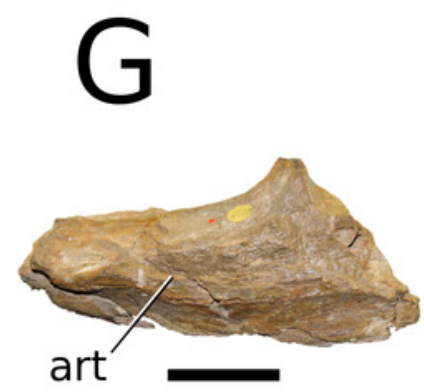


\section{Figure 5}

Chinese teleosauroid previously referred to as Peipehsuchus.

Figure 5. The Chinese teleosauroid previously referred to as Peipehsuchus (see Li, 1993), IVPP V 10098, holotype. Skull in (A) dorsal and (B) ventral (palatal) views. Refer to abbreviations list. Scale bars: $10 \mathrm{~cm}$.

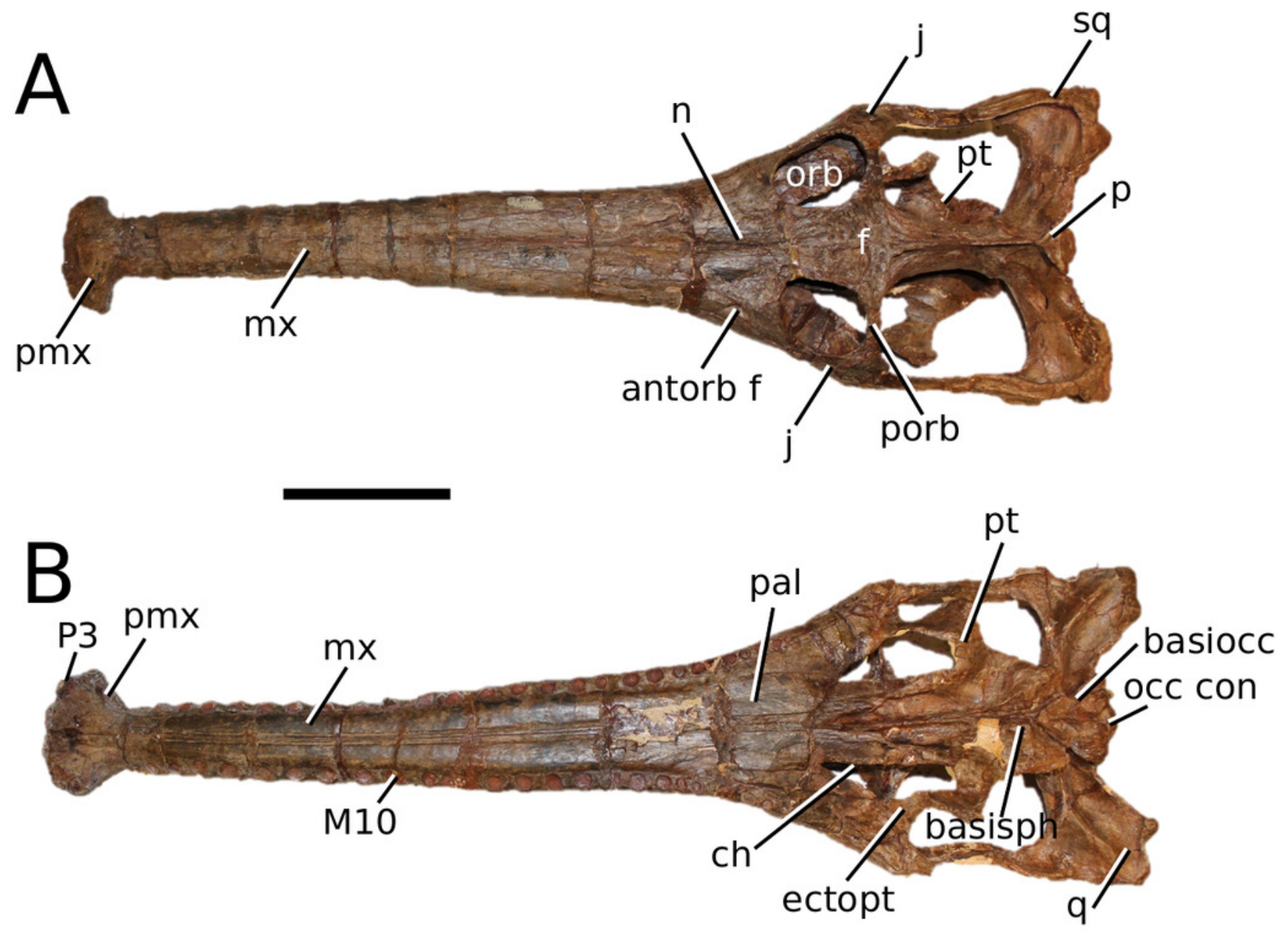




\section{Figure 6}

Platysuchus multiscrobiculatus.

Figure 6. Platysuchus multiscrobiculatus (Berckhemer, 1929) Westphal, 1961, SMNS 9930, holotype. (A) Nearly complete skeleton, with close-up views of (B) the skull, (C) forelimb, (D) trunk region and $(E)$ hindlimb. Refer to abbreviations list. Not to scale.

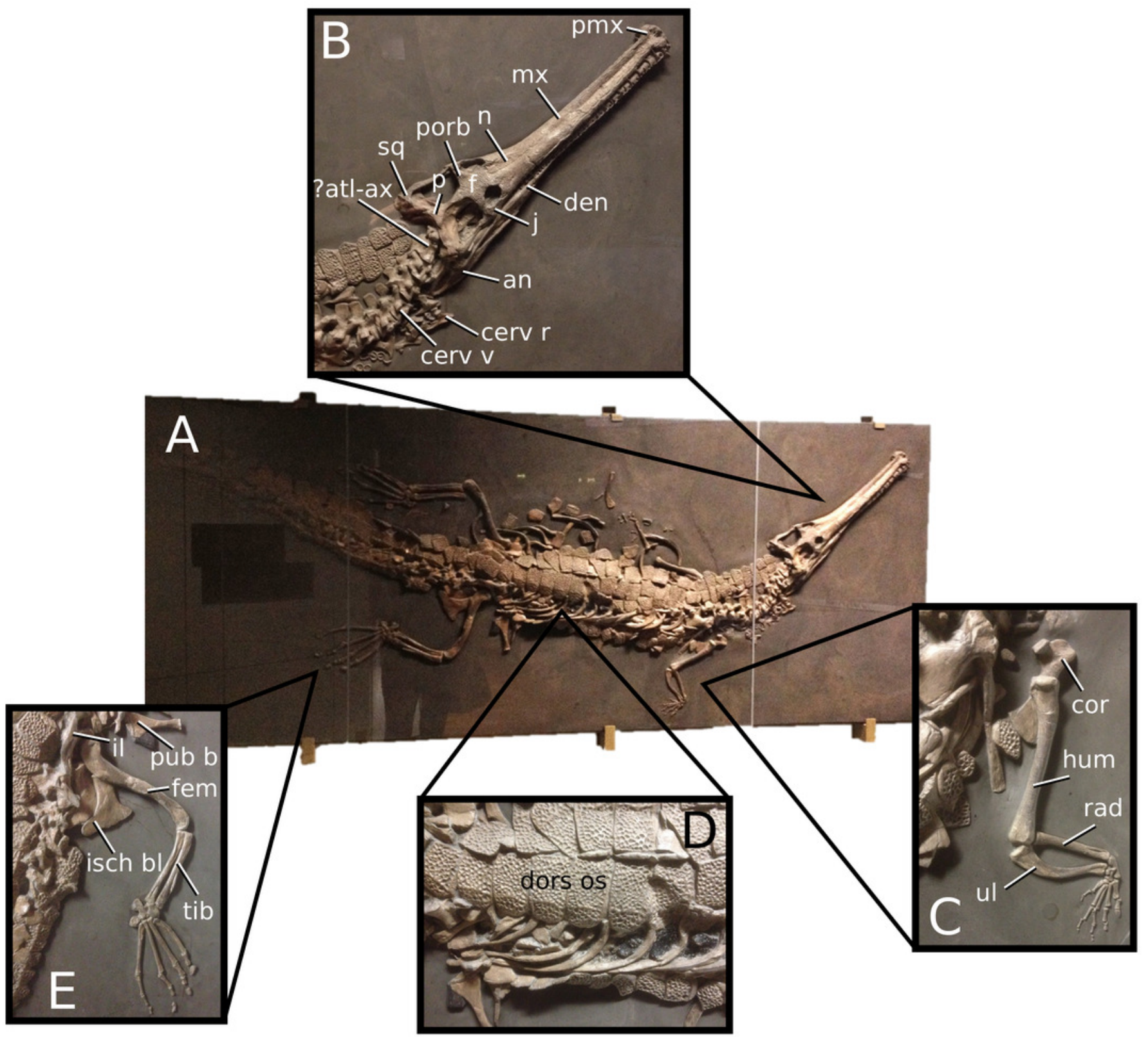


Figure 7

Teleosaurus cadomensis.

Figure 7. Teleosaurus cadomensis (Lamouroux, 1820), MNHN AC 8746, neotype. Partial skull in (A) dorsal, (B) ventral (palatal), (C) left lateral, (D) right lateral and (E) occipital views. Refer to abbreviations list. Scale bars: $5 \mathrm{~cm}$.
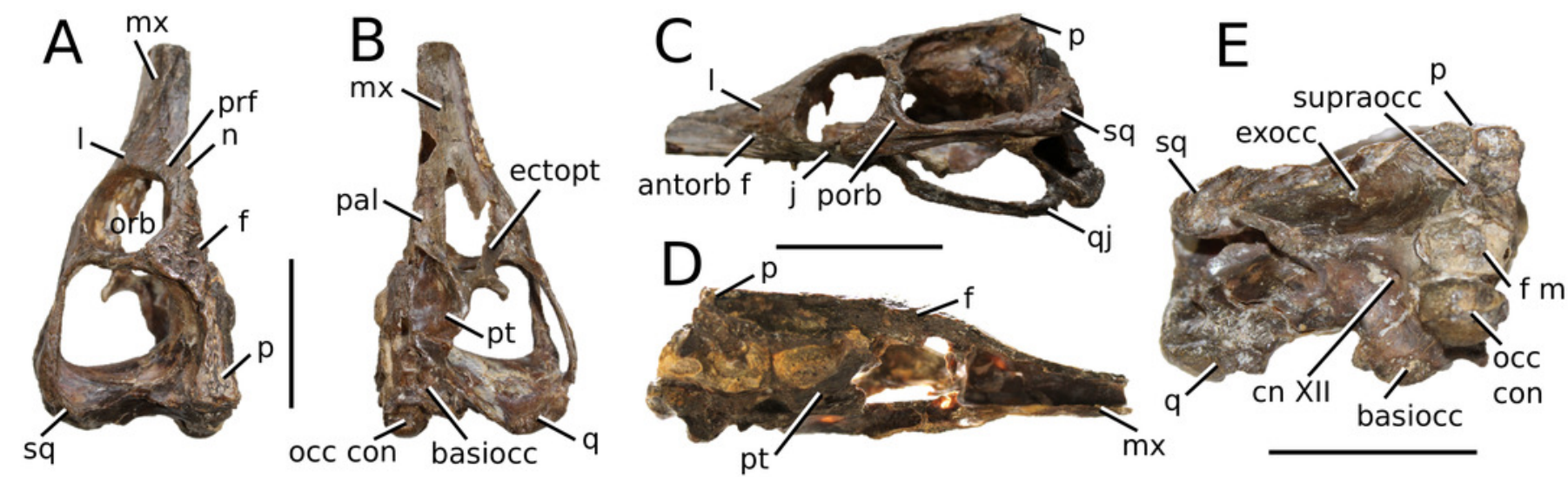
Figure 8

Mycterosuchus nasutus.

Figure 8. Mycterosuchus nasutus Andrews, 1913, NHMUK PV R 2617, holotype. Skull in (A) dorsal and (B) ventral (palatal) views, and dentary in (C) dorsal view. Note the extremely rugose dorsal cranium. Refer to abbreviations list. Scale bars: $10 \mathrm{~cm}$. 


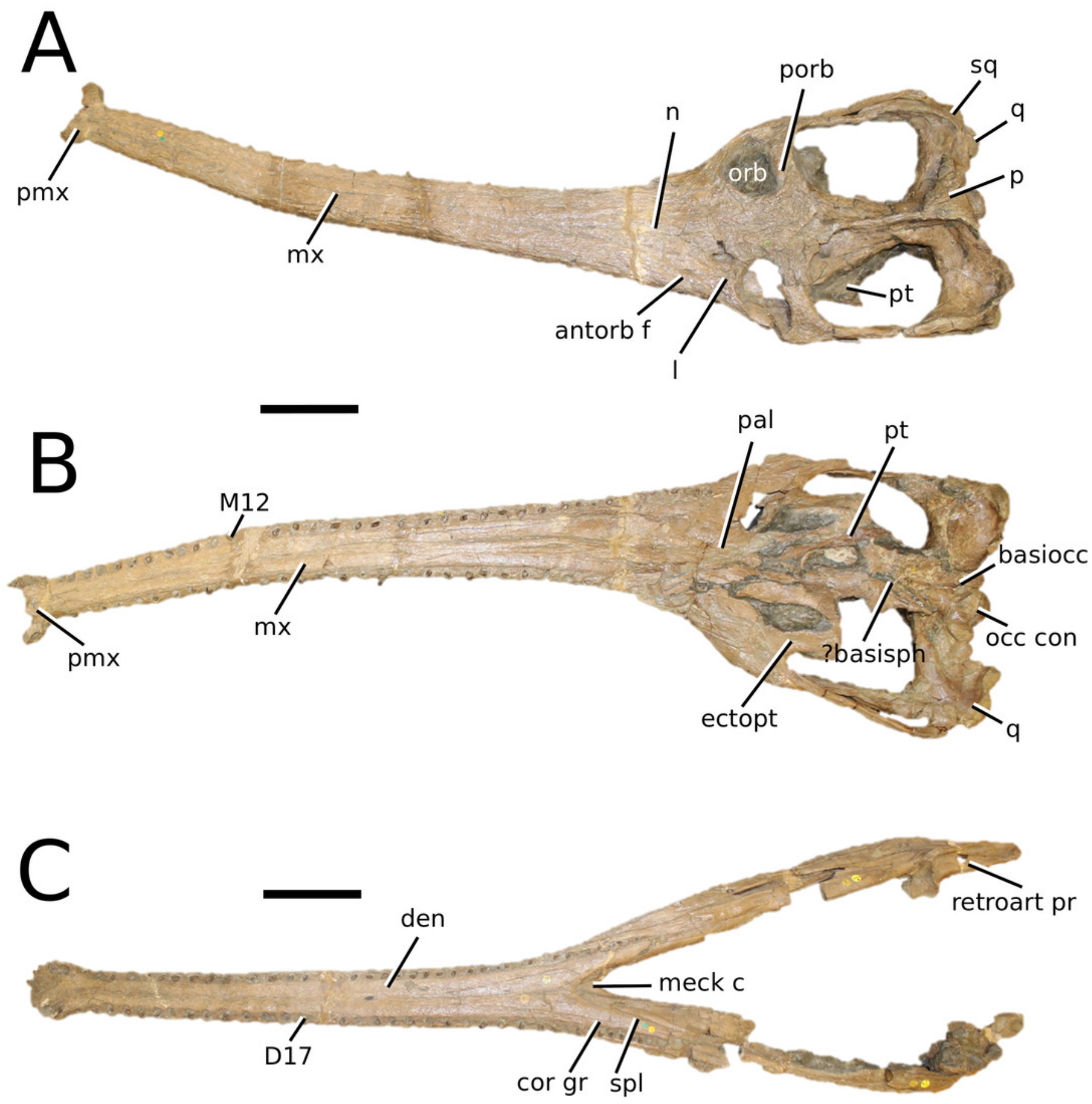




\section{Figure 9}

Aeolodon priscus.

Figure 9. Aeolodon priscus (von Sömmering, 1814), (A-E) NHMUK PV R 1086, holotype and (F) MNHN.F.CNJ 78, referred specimen (modified from Figure 10 in Foffa et al. (2019)). (A) Partial skeleton with close-ups of (B) the skull, (C) hindlimb, (D) trunk region and (E) pelvic area. (F) Nearly complete skeleton. Scale bars: $10 \mathrm{~cm}(\mathrm{~A})$ and $3 \mathrm{~cm}$ (B-E), (F) not to scale. 

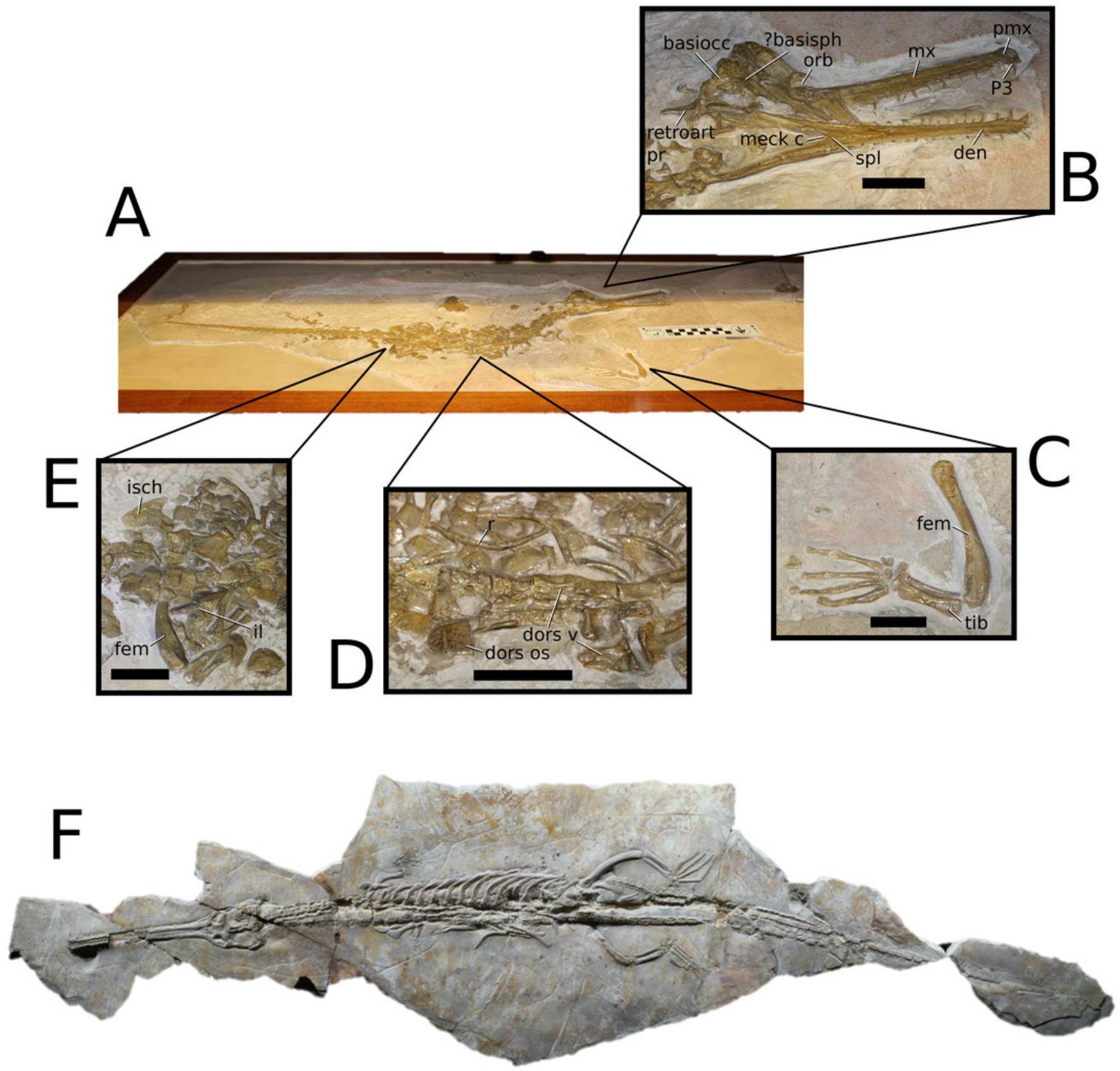


\section{Figure 10}

Bathysuchus megarhinus.

Figure 10. Bathysuchus megarhinus (Hulke, 1871) Foffa et al., 2019. (A-D) NHMUK PV OR 43086, holotype; (E-G) unnumbered LPP specimen. In (A, E) dorsal, (B) ventral, (C) right lateral, (D, F) left lateral and (G) occipital views. Refer to abbreviations list. Scale bars: 10 $\mathrm{cm}$. 

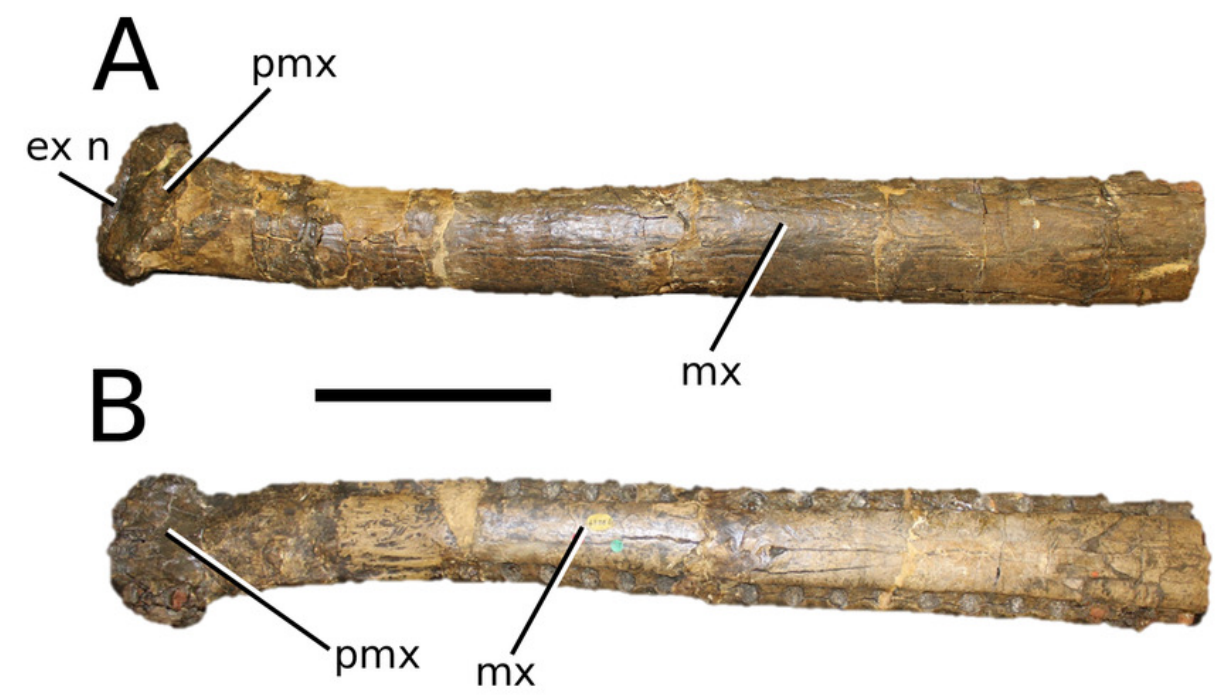

C
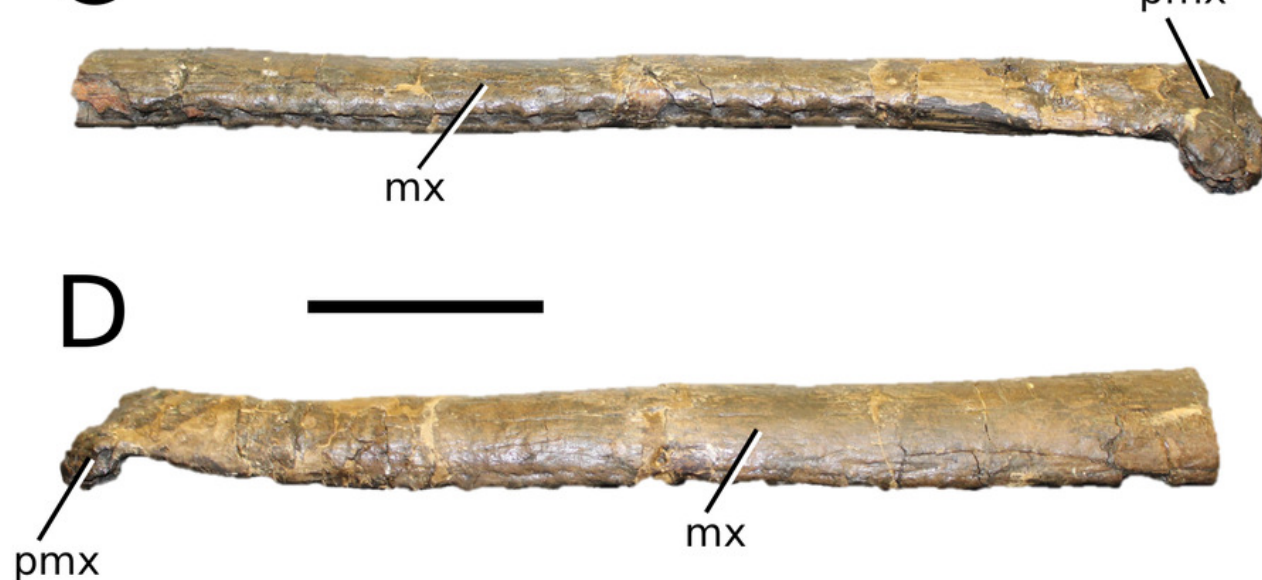

$\mathrm{pmx}$
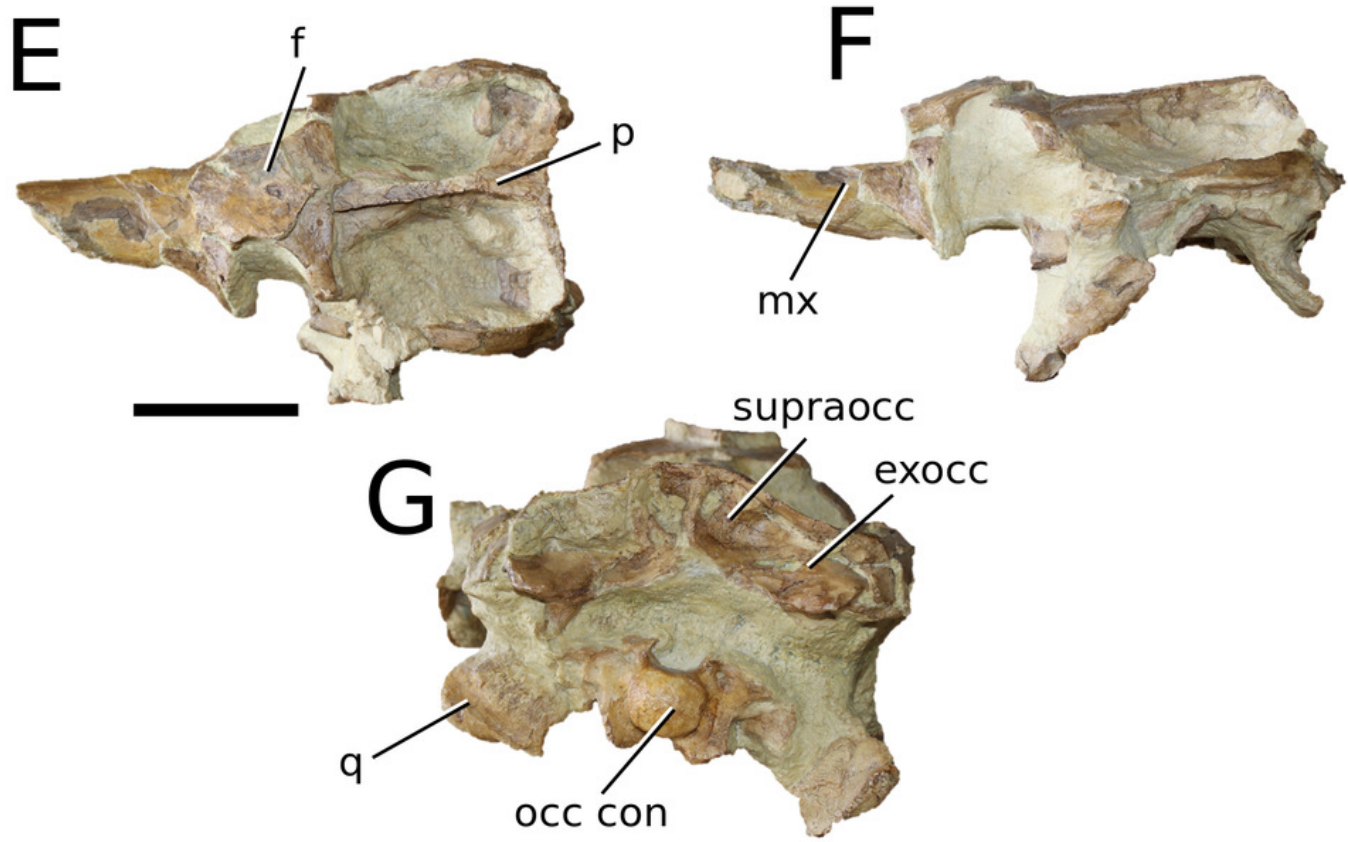
Figure 11

Sericodon jugleri.

Figure 11. Sericodon jugleri von Meyer, 1845, referred specimens. (A) Tooth in lingual view (SMF R 4318) and (B) anterior rostrum in dorsal view (LMH 16646). Refer to abbreviations list.

Scale bars: $1 \mathrm{~cm} \mathrm{(A)} \mathrm{and} 5 \mathrm{~cm}(B)$.
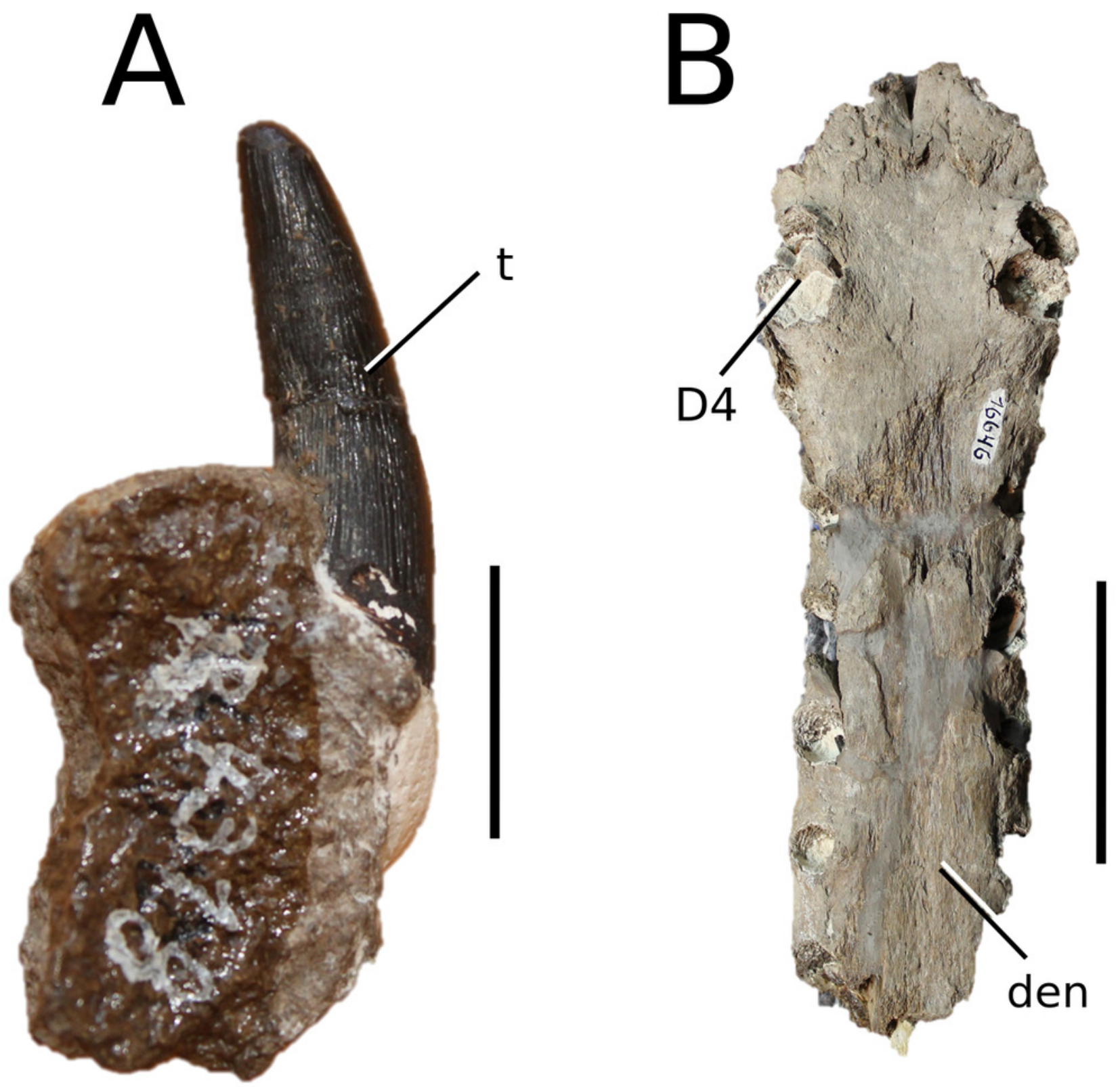


\section{Figure 12}

Indosinosuchus potamosiamensis.

Figure 12. Indosinosuchus potamosiamensis Martin et al., 2019, PRC-11, holotype. Skull and attached mandible in (A) dorsal and (B) ventral (palatal) views. Refer to abbreviations list. Scale bar: $10 \mathrm{~cm}$.

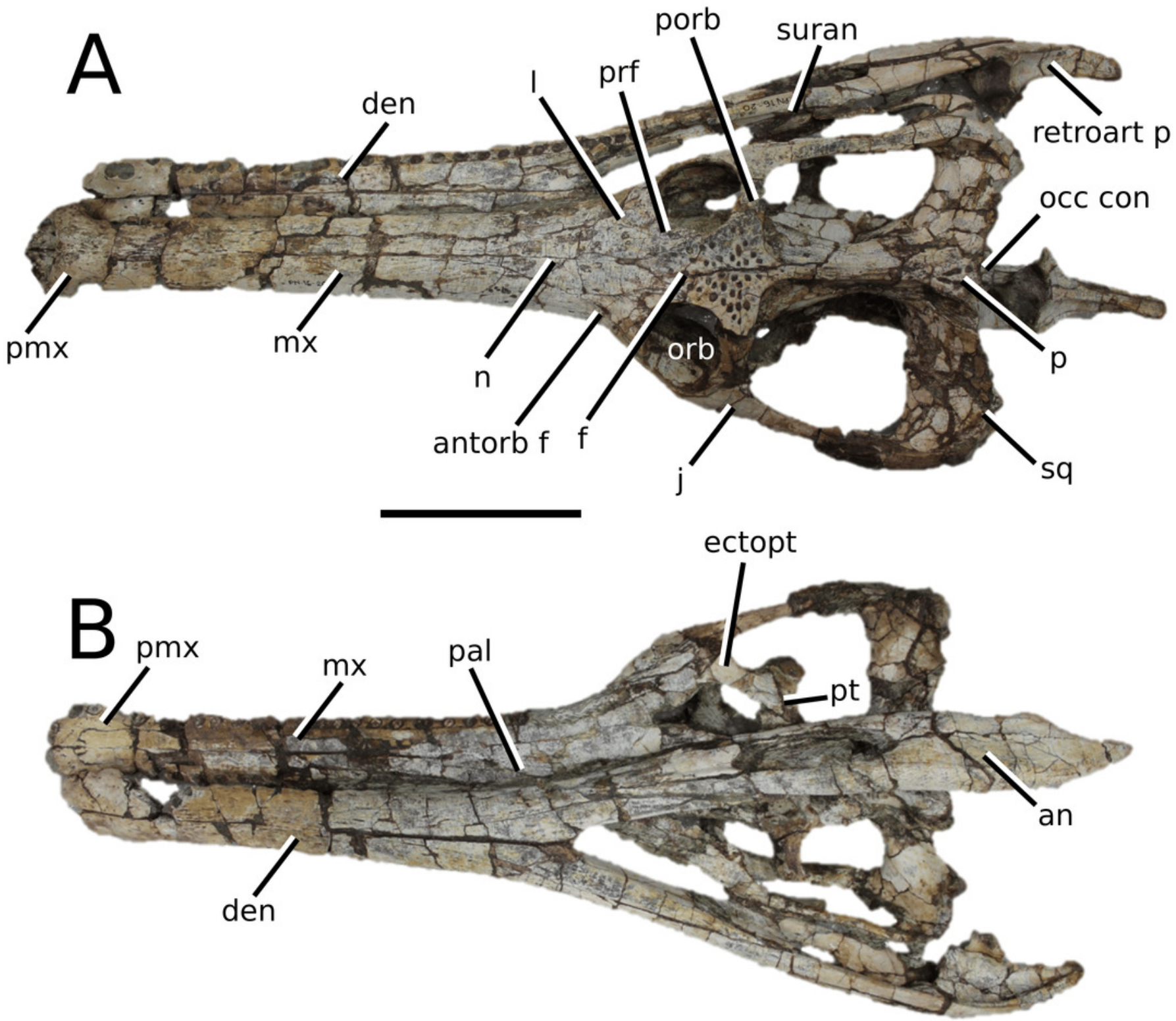


Figure 13

Indosinosuchus kalasinensis.

Figure 13. Indosinosuchus kalasinensis, sp. nov., PRC-239. Skull and mandible in (A) dorsal and (B) right lateral views. Refer to abbreviations list. Scale bar: $10 \mathrm{~cm}$.
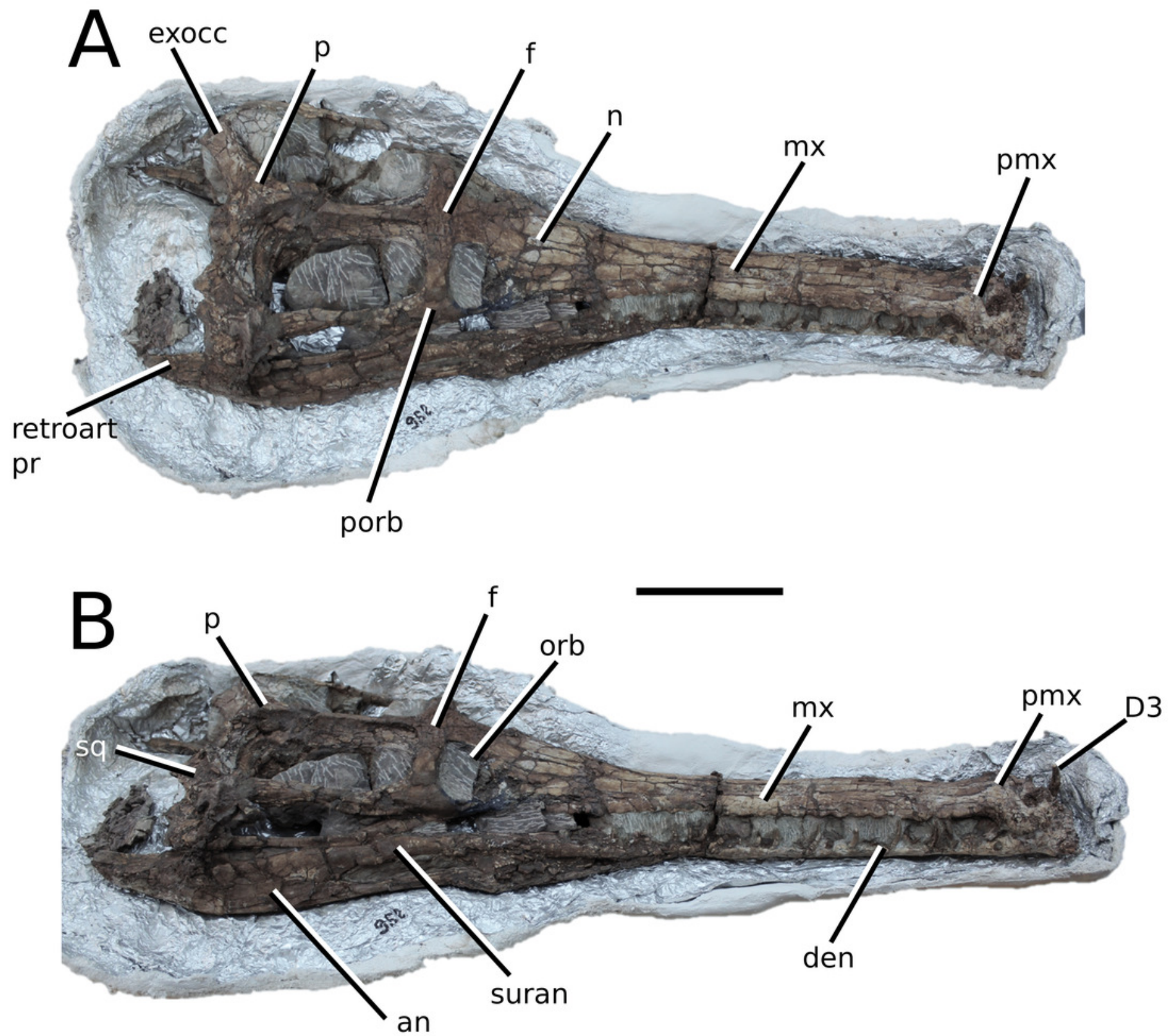
Figure 14

Macrospondylus bollensis.

Figure 14. Macrospondylus bollensis (von Jäger, 1828). (A) MMG BwJ 595, holotype, partial postcranial skeleton. (B) Complete skeleton MMG BwJ 565. Refer to abbreviations list. Scale bars: $10 \mathrm{~cm}$. 

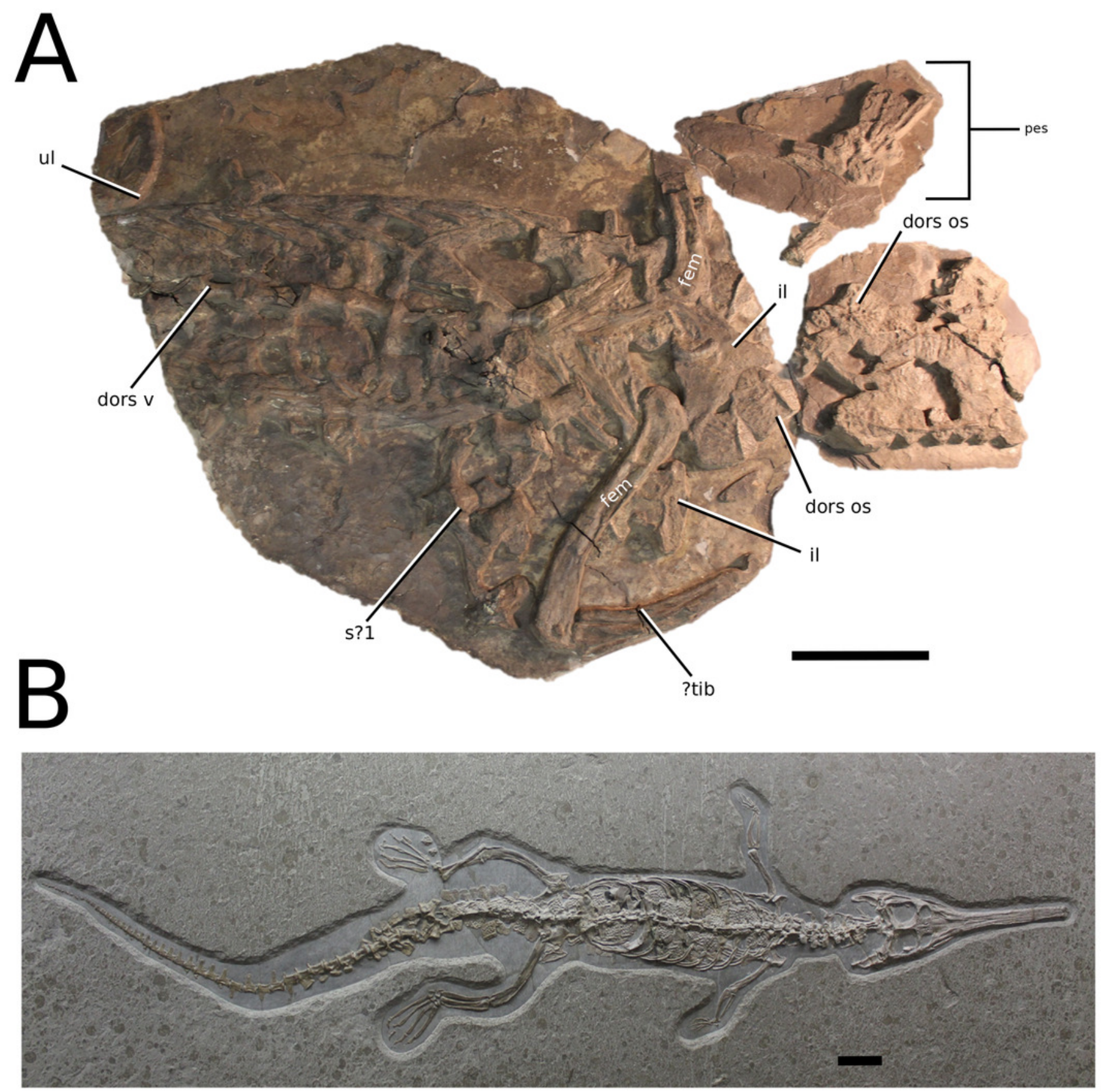


\section{Figure 15}

Seldsienean megistorhynchus.

Figure 15. Seldsienean megistorhynchus (Eudes-Deslongchamps, 1866a), comb. nov., MMT P28-1, neotype. Skull in (A) dorsal, (B) ventral (palatal), (C) right lateral and (D) left lateral views. Mandible in (E) dorsal view. Refer to abbreviations list. Scale bars: $10 \mathrm{~cm}$. Photographs provided by V. Lamarque.
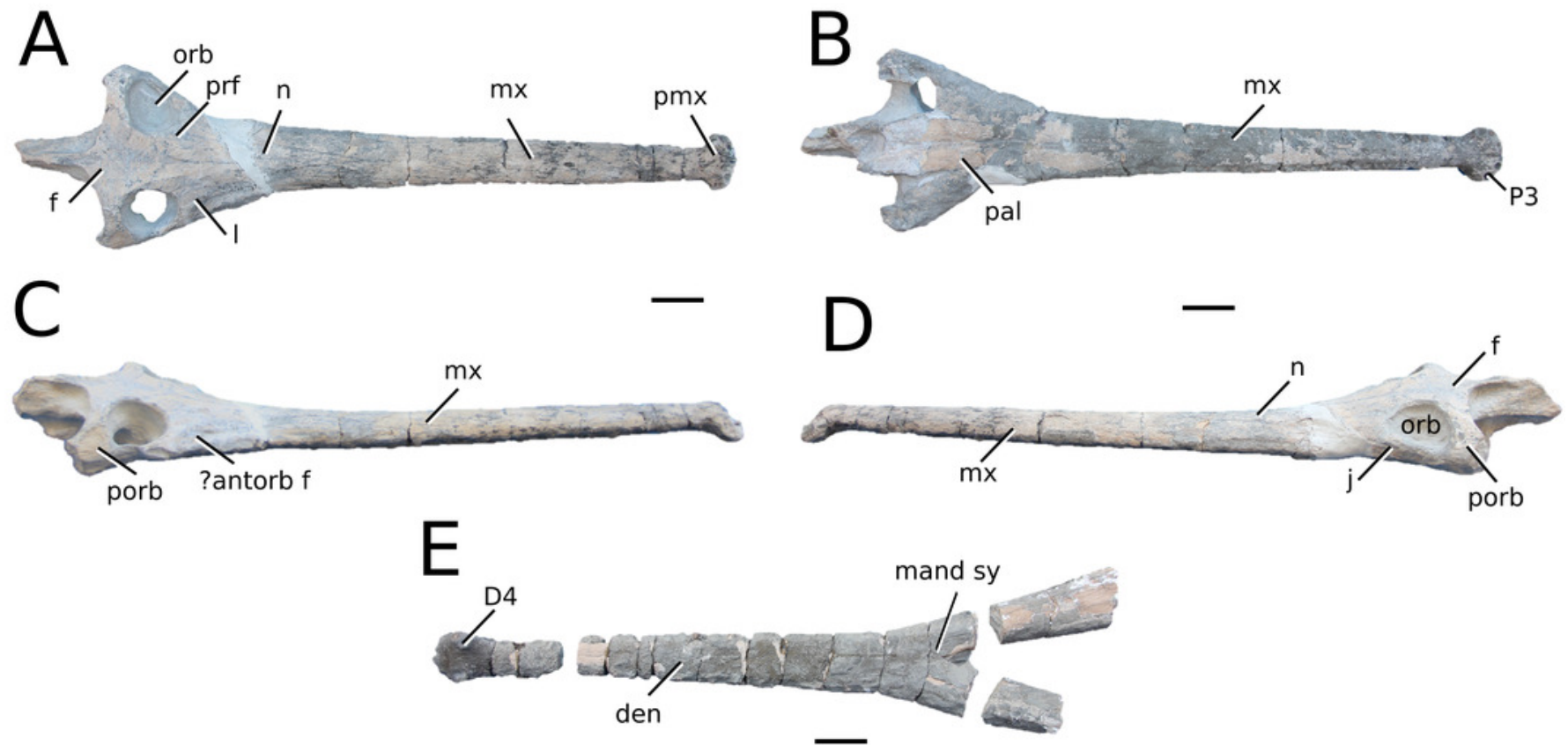


\section{Figure 16}

Charitomenosuchus leedsi.

Figure 16. Charitomenosuchus leedsi (Andrews, 1913), comb. nov., NHMUK PV R 3320, holotype. Skull in (A) dorsal, (B) ventral (palatal) and (C) right lateral views; partial mandible in (D) dorsal view. (E) Posterior section of the mandible in right lateral view; atlas in (F) anterior and (G) right lateral view. Refer to abbreviations list. Scale bars: $10 \mathrm{~cm}(\mathrm{~A}-\mathrm{D})$ and 2 cm (E-G).
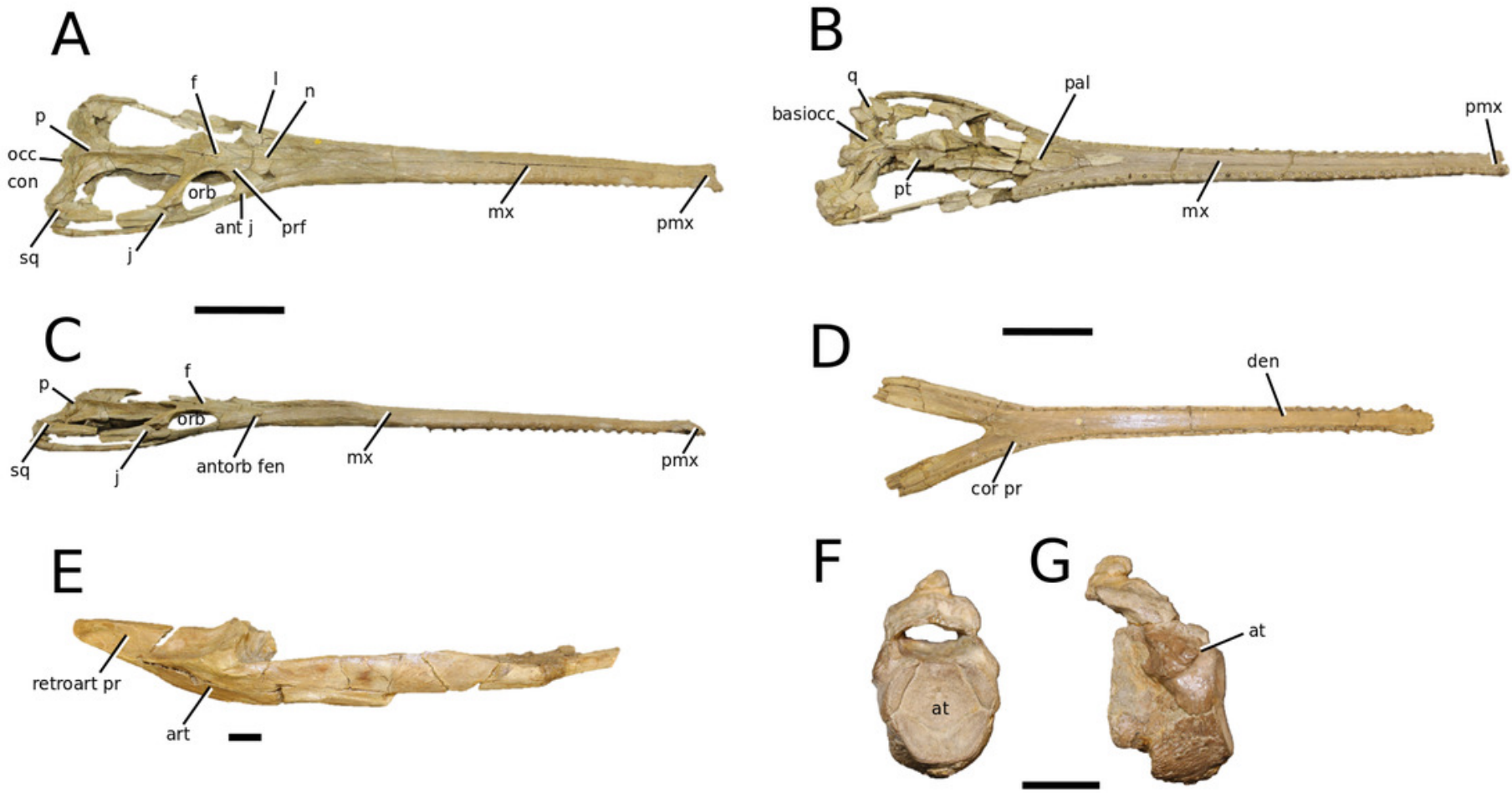


\section{Figure 17}

Deslongchampsina larteti.

Figure 17. Deslongchampsina larteti (Eudes-Deslongchamps, 1866a) Johnson, Young \& Brusatte, 2019, OUMNH J.29851, neotype. Skull in (A) dorsal, (B) ventral (palatal), (C) right lateral, (D) left lateral and (E) occipital views. Refer to abbreviations list. Scale bars: $5 \mathrm{~cm}$.

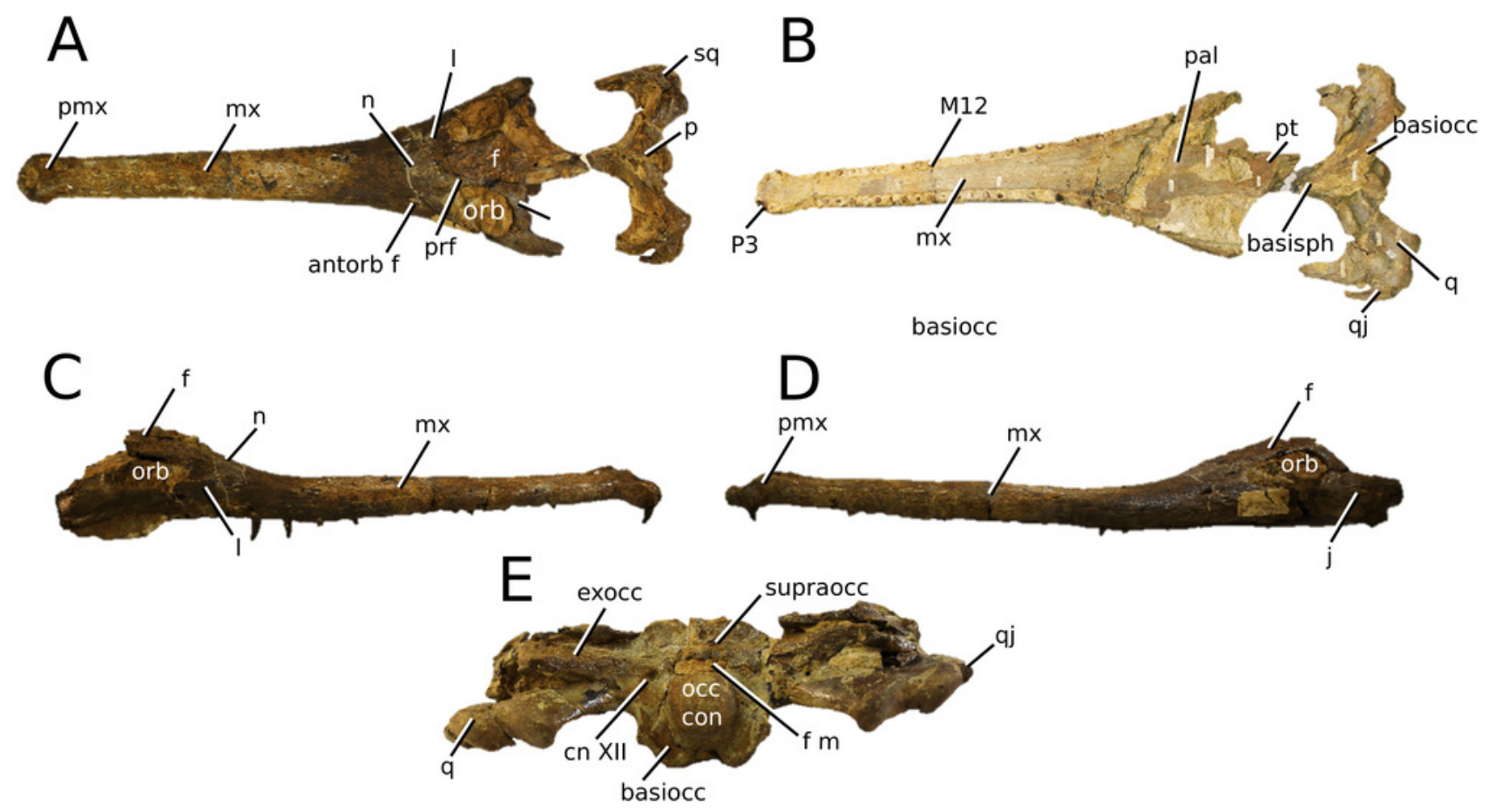


Figure 18

Proexochokefalos heberti.

Figure 18. Proexochokefalos heberti (Morel de Glasville, 1876), comb. nov., MNHN.F 1890-13, holotype. Skull in (A) dorsal, (B) left lateral and (C) occipital views. Refer to abbreviations list. Scale bars: $10 \mathrm{~cm}$.
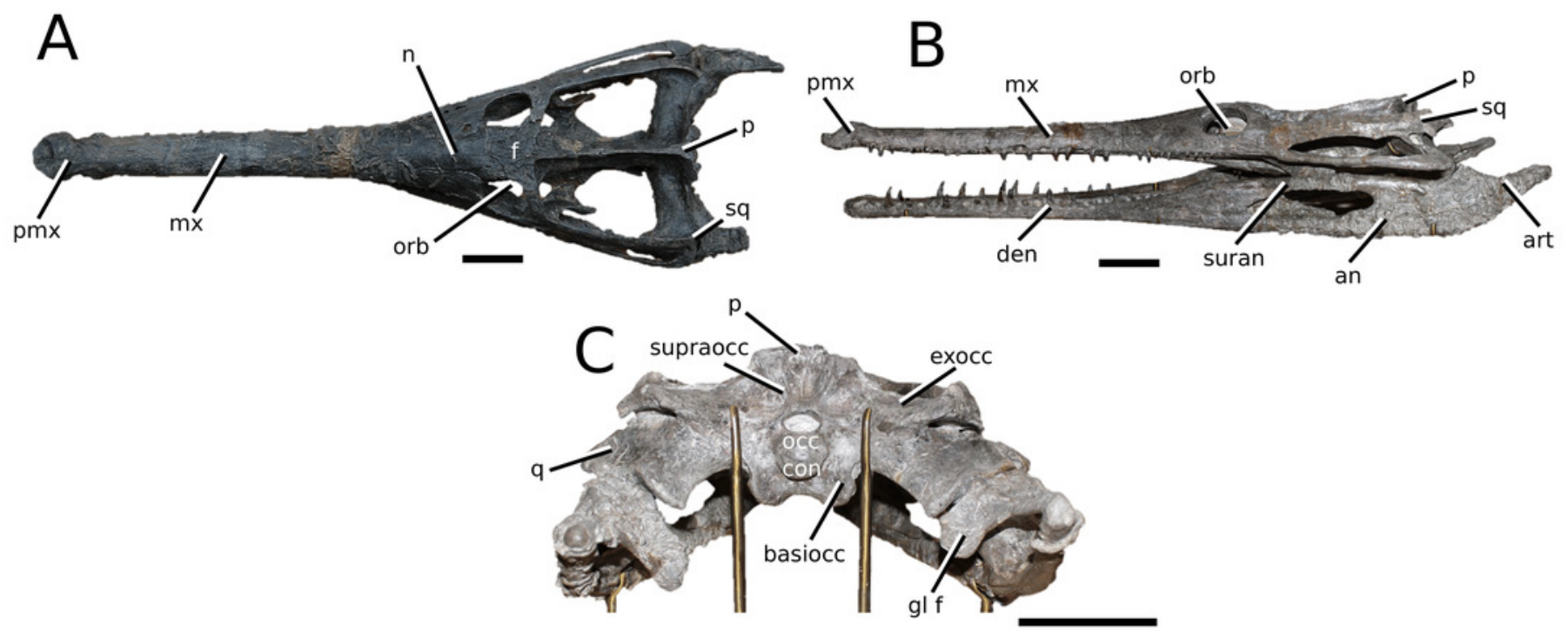


\section{Figure 19}

Proexochokefalos cf. bouchardi.

Figure 19. Proexochokefalos cf. bouchardi (Sauvage, 1872), comb. nov. Unknown specimen number, photo provided by Y. Lepage (from Lepage et al., 2008). Skull in dorsal view. Refer to abbreviations list. Scale bar: $10 \mathrm{~cm}$.

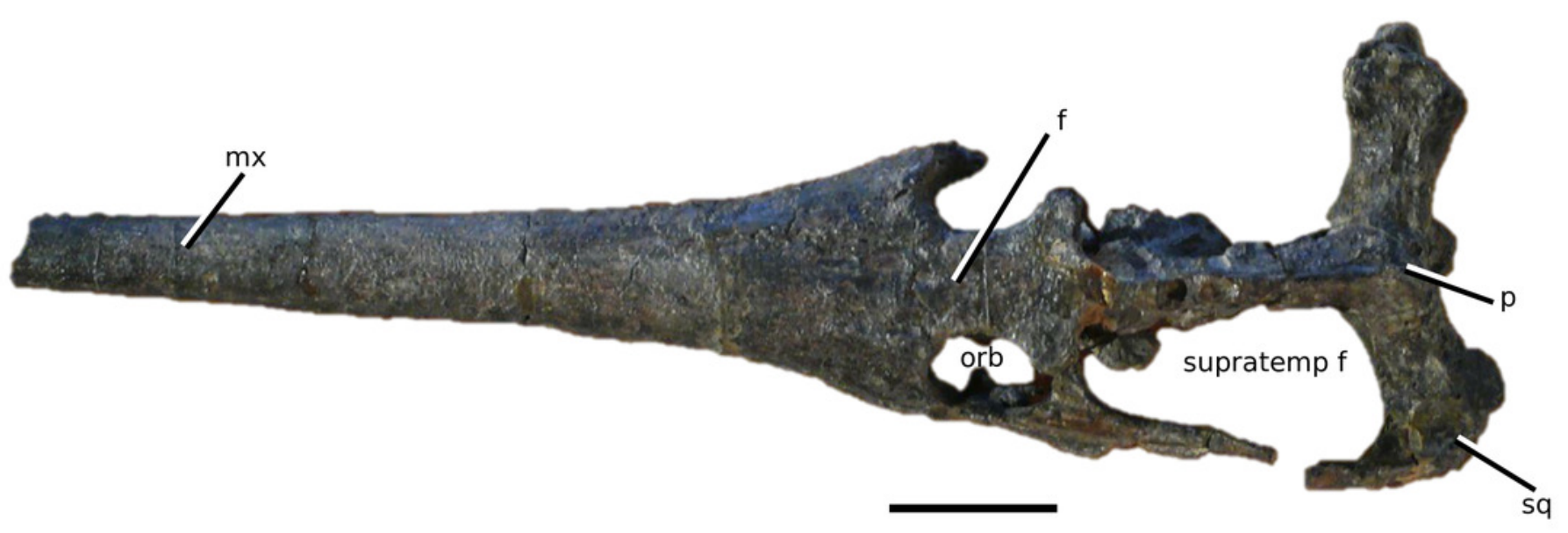




\section{Figure 20}

Steneosaurus rostromajor.

Figure 20. Steneosaurus rostromajor (Geoffroy Saint-Hilaire, 1825), MNHN.RJN 134c-d, nomen dubium. Partial rostrum in (A) dorsal, (B) ventral and (C) left lateral views. Refer to abbreviations list. Scale bar: $10 \mathrm{~cm}$. 

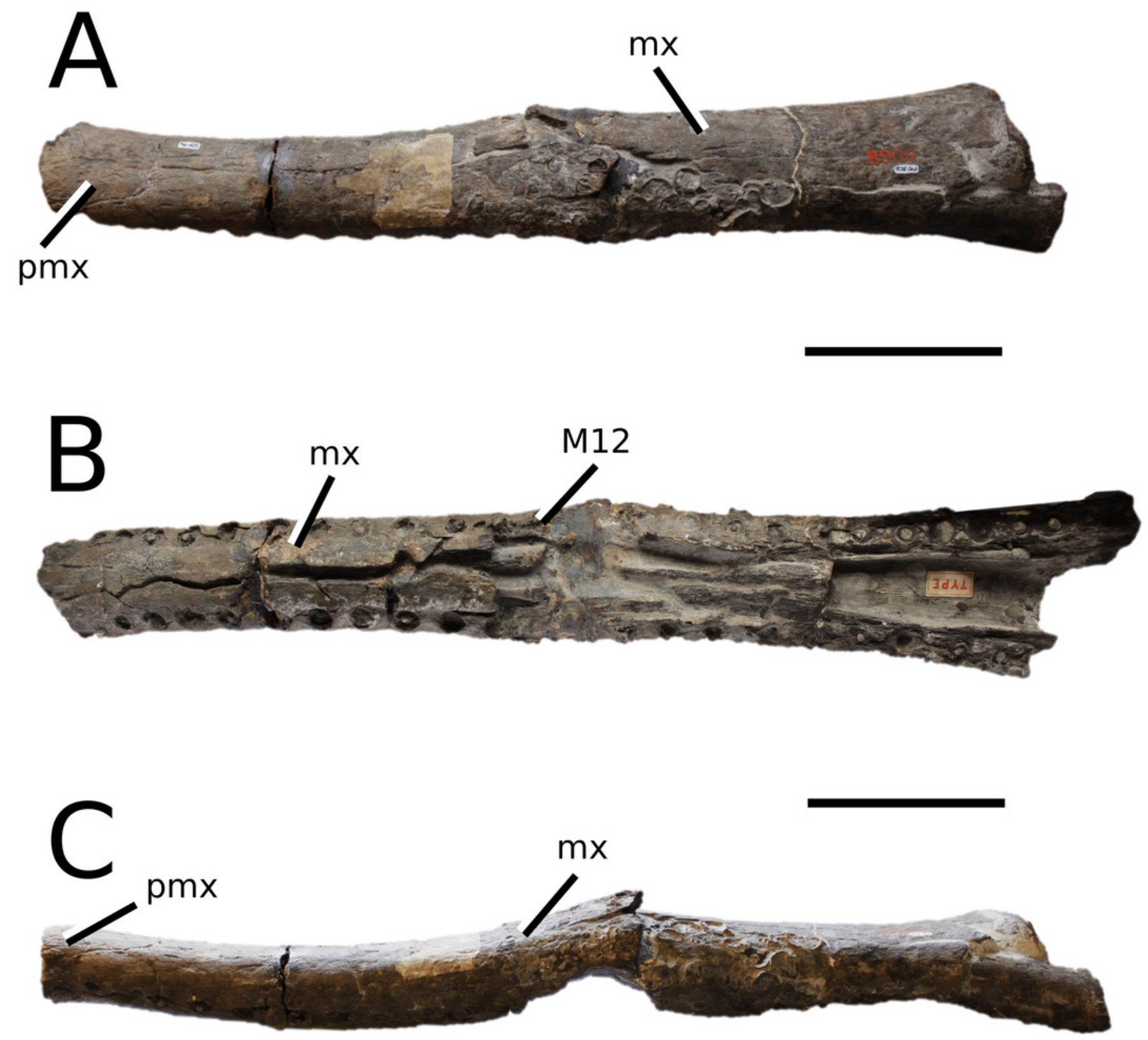


\section{Figure 21}

Andrianavoay baroni.

Figure 21. Andrianavoay baroni (Newton, 1893), comb. nov., NHMUK PV R 1999, holotype. Photograph of the partial skull and mandible in (A) right lateral view, as well as (B) partial rostrum in dorsal view; posterior skull in (C) dorsal and (D) ventral views; (E) partial mandible in dorsal view; and (F) fragment of osteoderm in dorsal view. Refer to abbreviations list.

Scale bars: $10 \mathrm{~cm}(A), 5 \mathrm{~cm}(B-E)$ and $3 \mathrm{~cm}(F)$. 


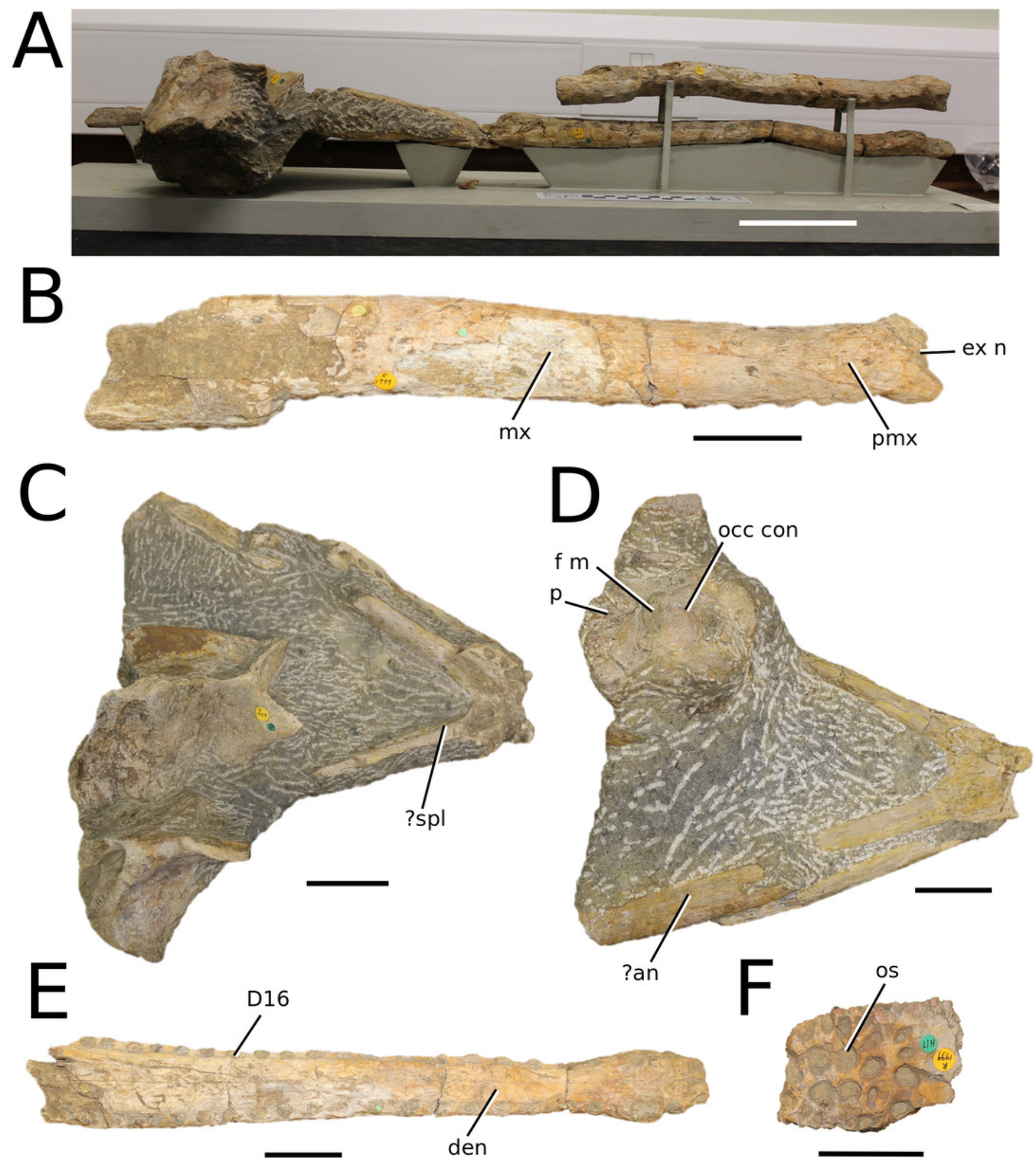




\section{Figure 22}

Neosteneosaurus edwardsi.

Figure 22. Neosteneosaurus edwardsi (Eudes-Deslongchamps, 1868a), comb. nov. (A-C) MNHN.RJN 118, holotype and (D-F) NHMUK PV R 2865, referred specimen. Partial skull in (A) dorsal, (B) ventral (palatal) and (C) right lateral views. Refer to abbreviations list. Scale bars: $10 \mathrm{~cm}$.
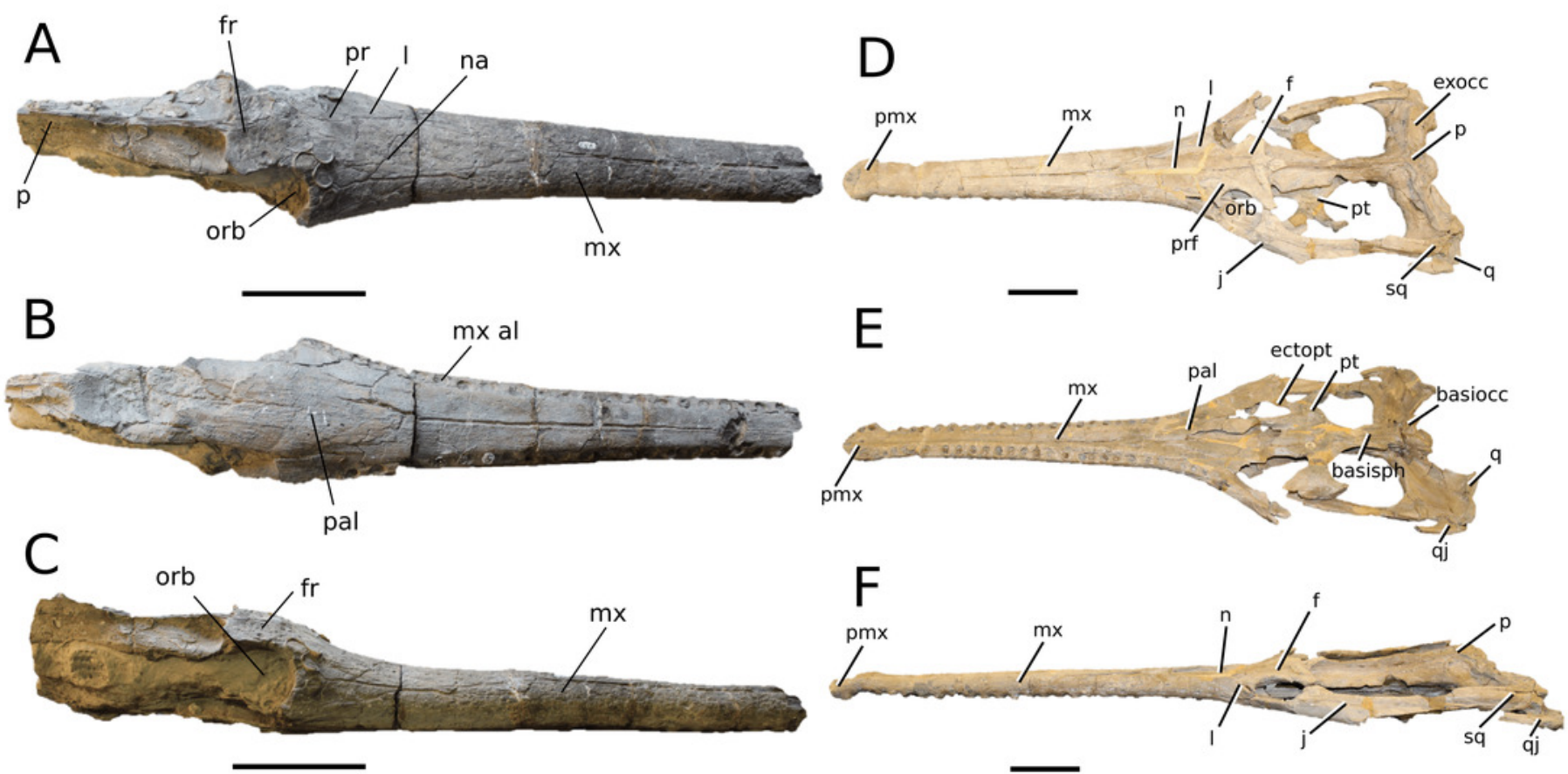


\section{Figure 23}

Yvridiosuchus boutilieri.

Figure 23. Yvridiosuchus boutilieri (Eudes-Deslongchamps, 1868c) Johnson, Young \& Brusatte, 2019. (A-D) OUMNH J.1401, holotype and (E-I) OUMNH J.29850, referred specimen.

Skull in (A, E) dorsal, (B, F) ventral (palatal), (C, G) right lateral, (D, H) left lateral and (I) occipital views. Refer to abbreviations list. Scale bars: $5 \mathrm{~cm}$. 

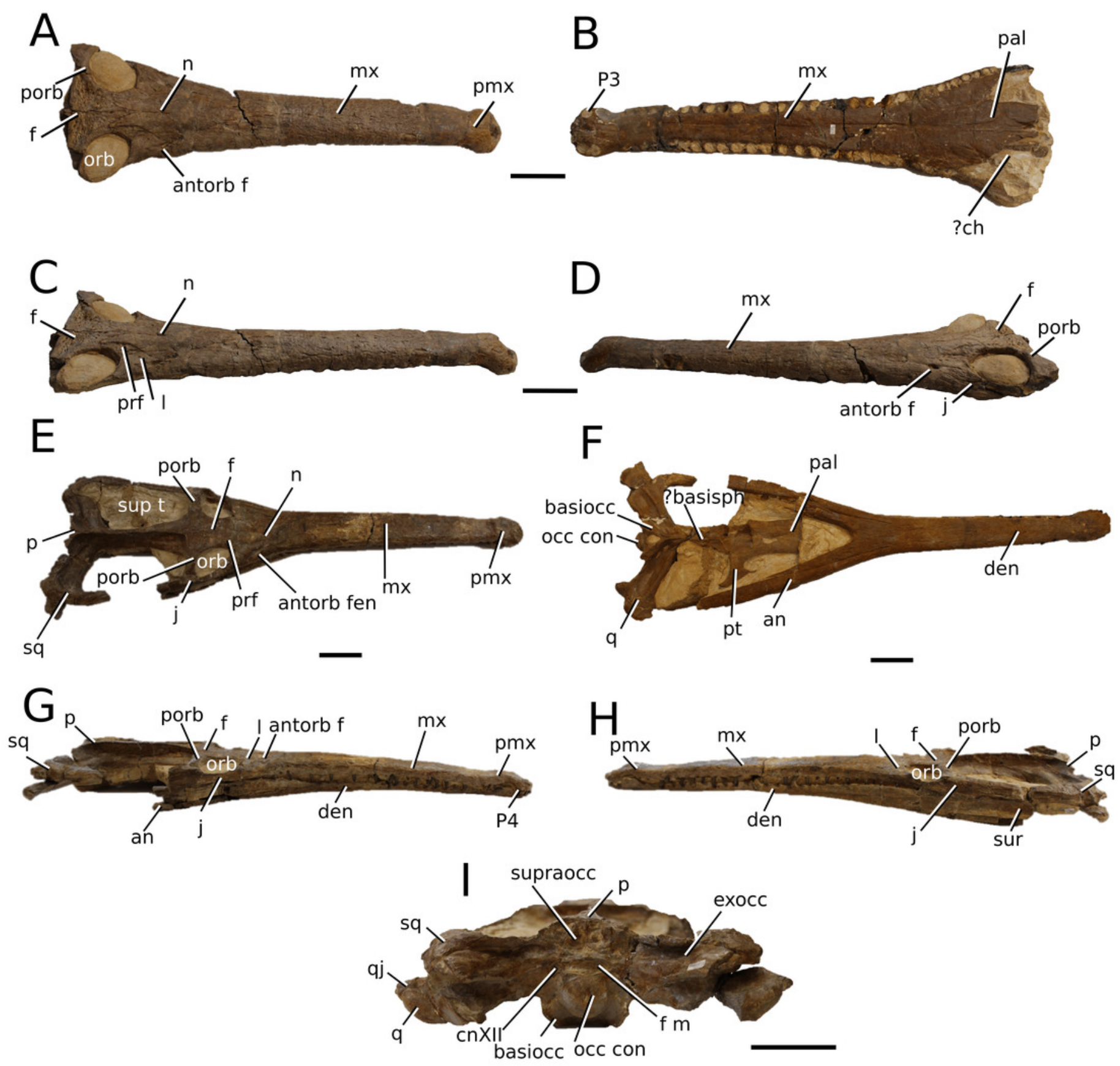


\section{Figure 24}

Lemmysuchus obtusidens.

Figure 24. Lemmysuchus obtusidens (Andrews, 1909) Johnson et al., 2017, NHMUK PV R 3168, holotype. Skull in (A) dorsal, (B) occipital, (C) right lateral and (D) left lateral views. Refer to abbreviations list. Scale bars: $20 \mathrm{~cm}$.
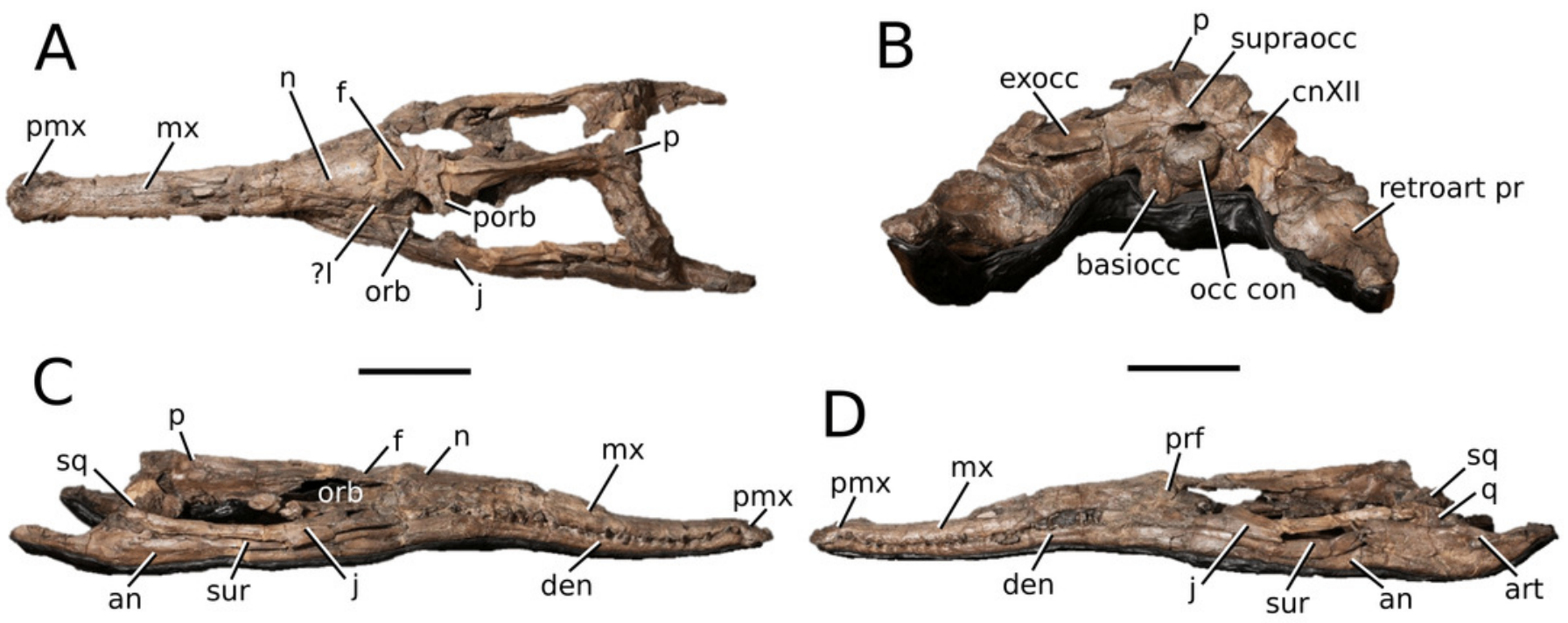


\section{Figure 25}

Machimosaurus buffetauti.

Figure 25. Machimosaurus buffetauti Young et al., 2015, SMNS 91415, holotype. Skull in (A) dorsal, (B) occipital, (C) right lateral and (D) left lateral views. Rostrum in (E) left lateral view, with a close-up of (i) the premaxillary alveoli. (F) Mandible in left lateral view. Refer to abbreviations list. Scale bars: $10 \mathrm{~cm}$. 

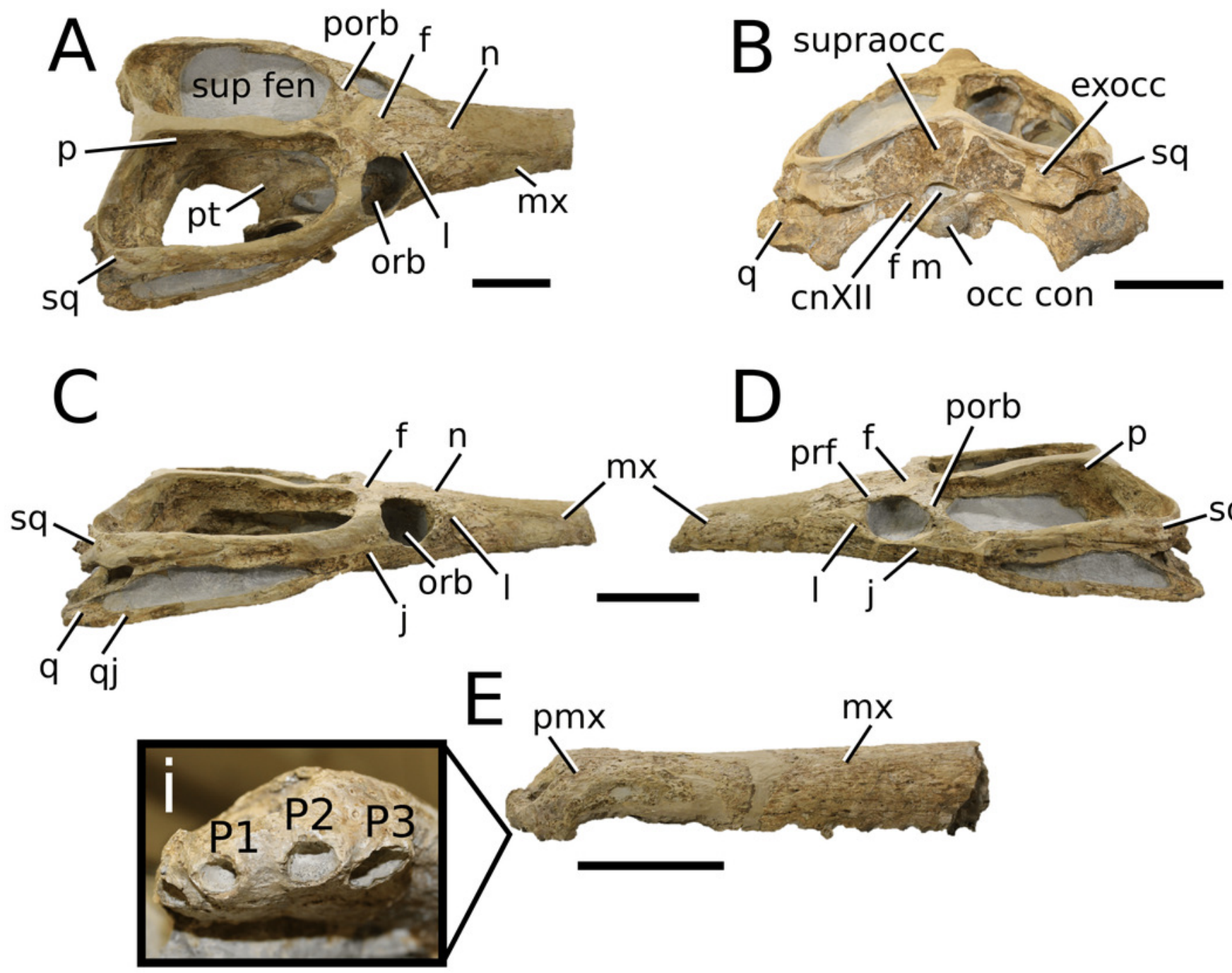

F

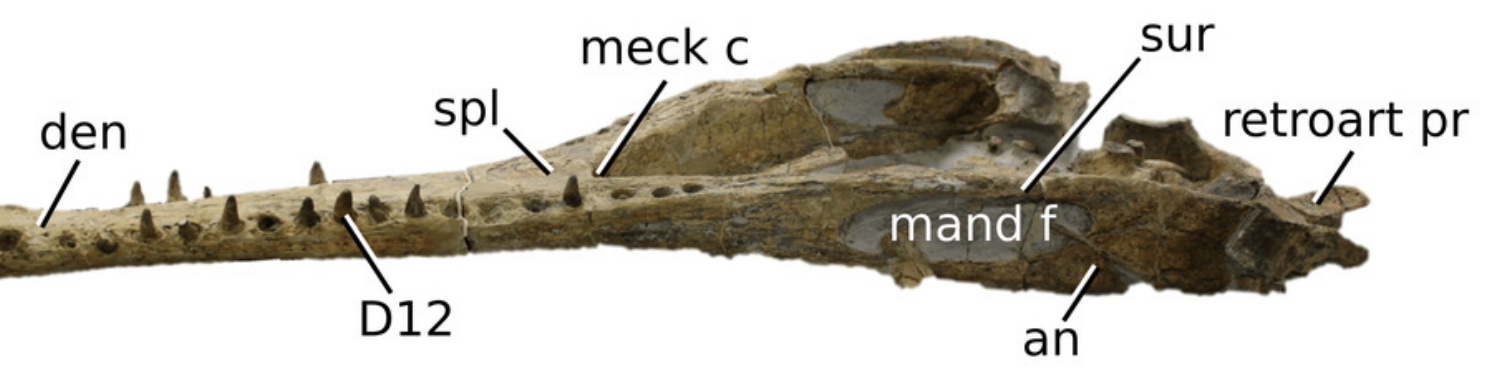


Figure 26

Machimosaurus mosae.

Figure 26. Machimosaurus mosae Sauvage \& Li $\square$ nard, 1879, IRSNB cast. Not to scale.

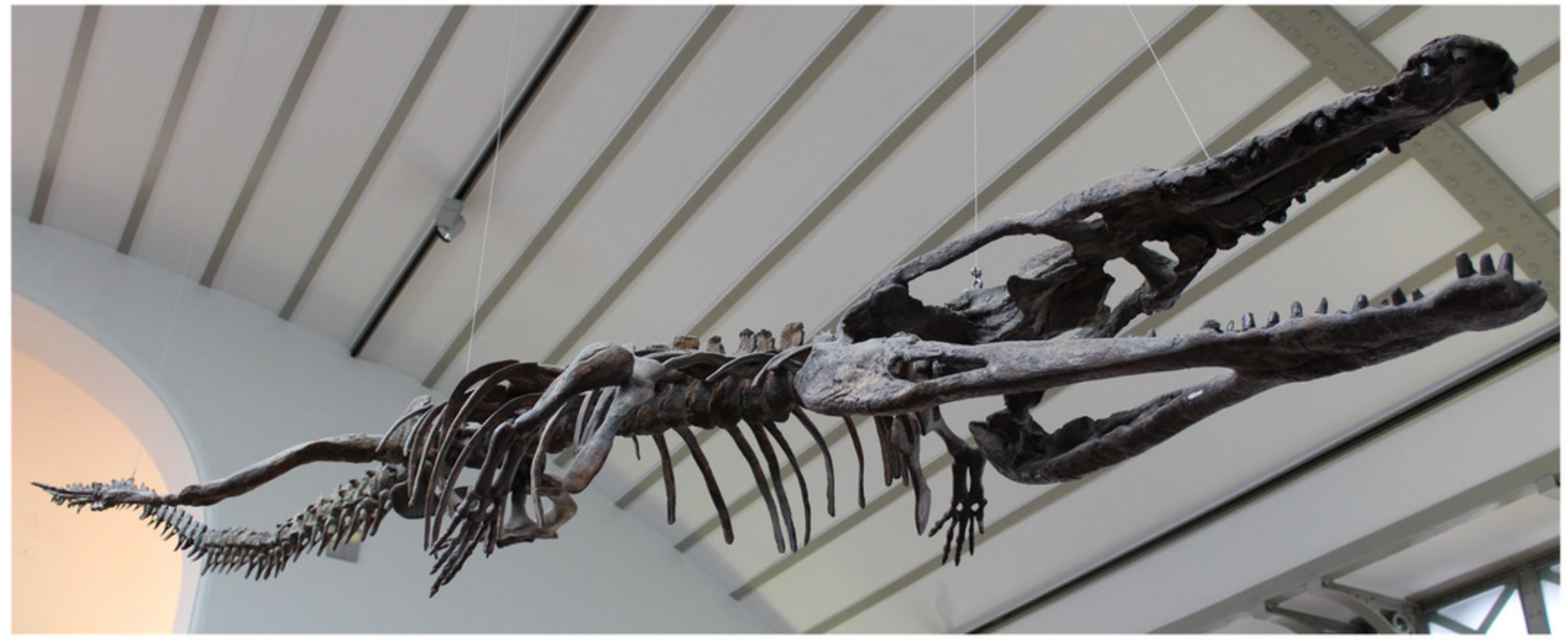




\section{Figure 27}

Machimosaurus hugii.

Figure 27. Machimosaurus hugii (von Meyer, 1837) emend. von Meyer, 1838, MG-8730, referred specimen. (A-C) MG-8730-2: occipital in (A) dorsal, (B) ventral and (C) occipital views. (D-E) MG-8730-1: partial rostrum in (D-E) palatal view. Refer to abbreviation list. Scale bars: $10 \mathrm{~cm}$.

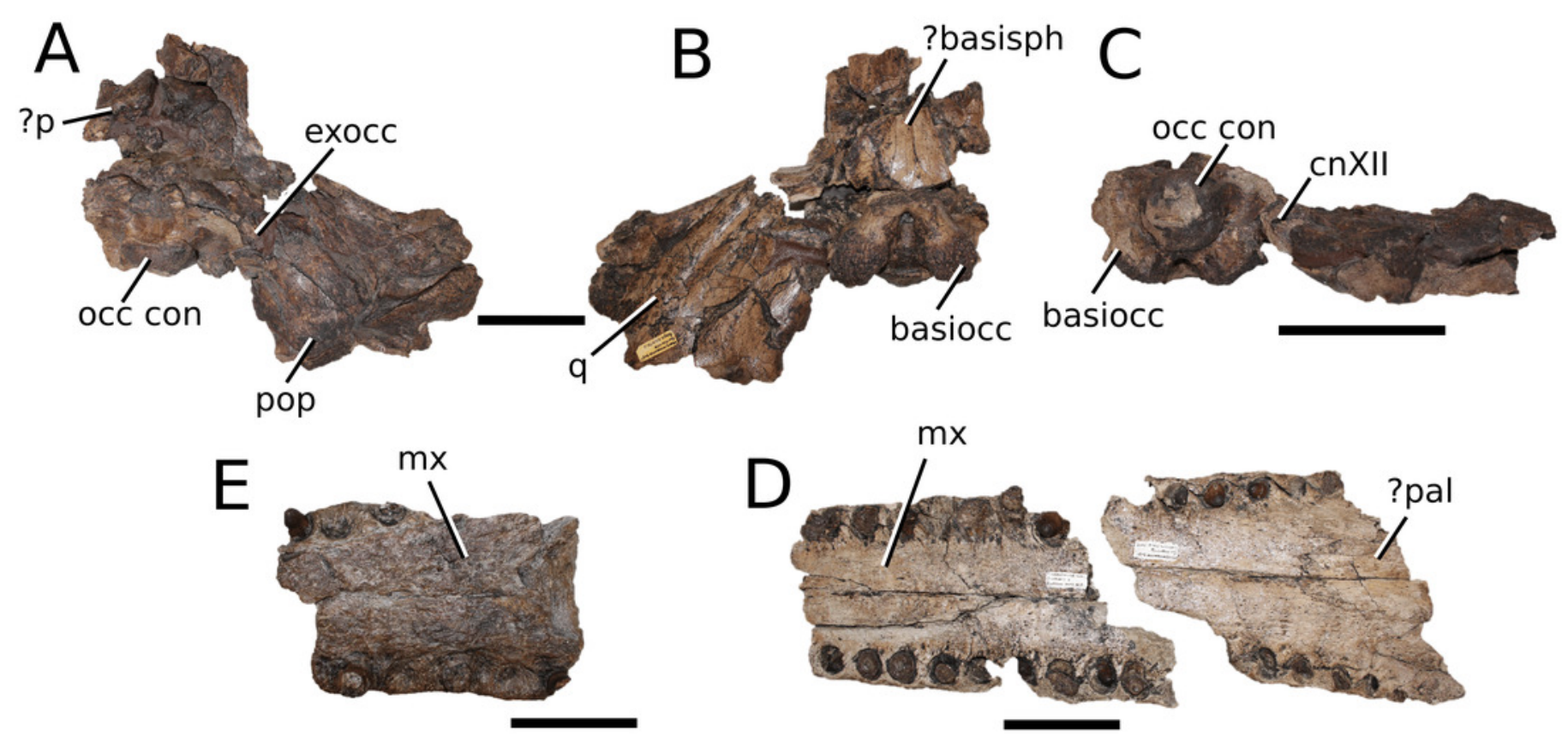




\section{Figure 28}

Machimosaurus rex.

Figure 28. Machimosaurus rex Fanti et al., 2016, ONM NG 1-25, holotype. Partial skull in (A) ventral view, with a close-up of the (i) maxillary alveoli. Additional material: (B) dorsal vertebra in anterior view; (C) dorsal osteoderm; and (D) close-up of tooth apex. Refer to abbreviation list. Scale bars: $10 \mathrm{~cm}$ (as indicated on A), $5 \mathrm{~cm}(B-C)$ and $1 \mathrm{~cm}(D)$.
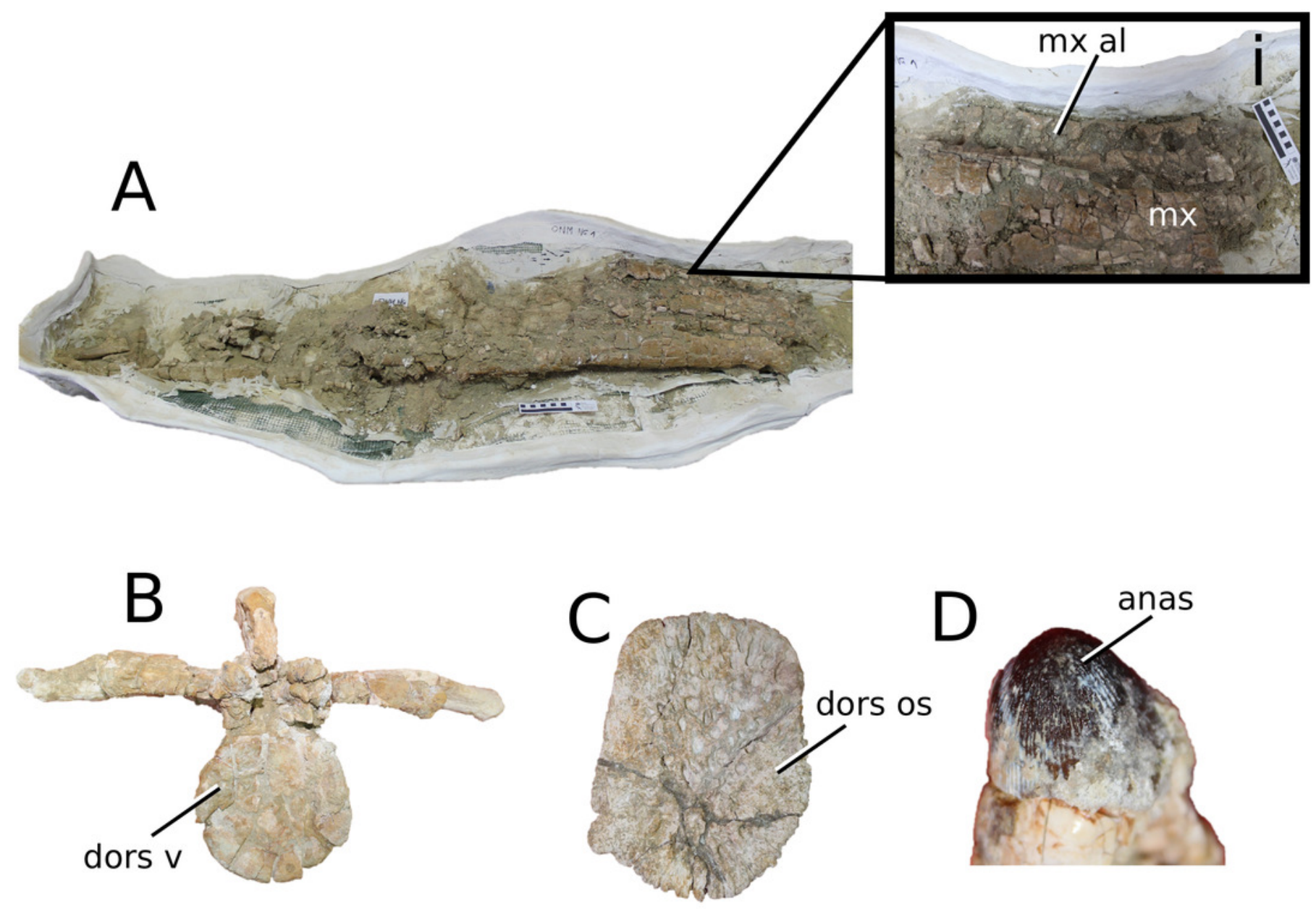


\section{Figure 29}

Comparative photographs: ornamentation on the prefrontal, lacrimal and frontal.

Figure 29. Comparative photographs displaying ornamentation on the prefrontal (ch. 12), lacrimal (ch. 13) and frontal (ch. 15) in dorsal view. (A) Plagiophthalmosuchus gracilirostris (NHMUK PV R 14892); (B) Clovesuurdameredeor stephani (NHMUK PV OR 49126); (C) Indosinosuchus potamosiamensis (PRC-11); (D) the Chinese teleosauroid (IVPP V 10098); (E) Mycterosuchus nasutus (NHMUK PV R 2617); (F) Charitomenosuchus leedsi (NHMUK PV R 38060; (G) Neosteneosaurus edwardsi (NHMUK PV R 2865); (H) Yvridiosuchus boutilieri (OUMNH J.1401); and (I) Machimosaurus buワetauti (SMNS 91415). Scale bars: $4 \mathrm{~cm}$. 

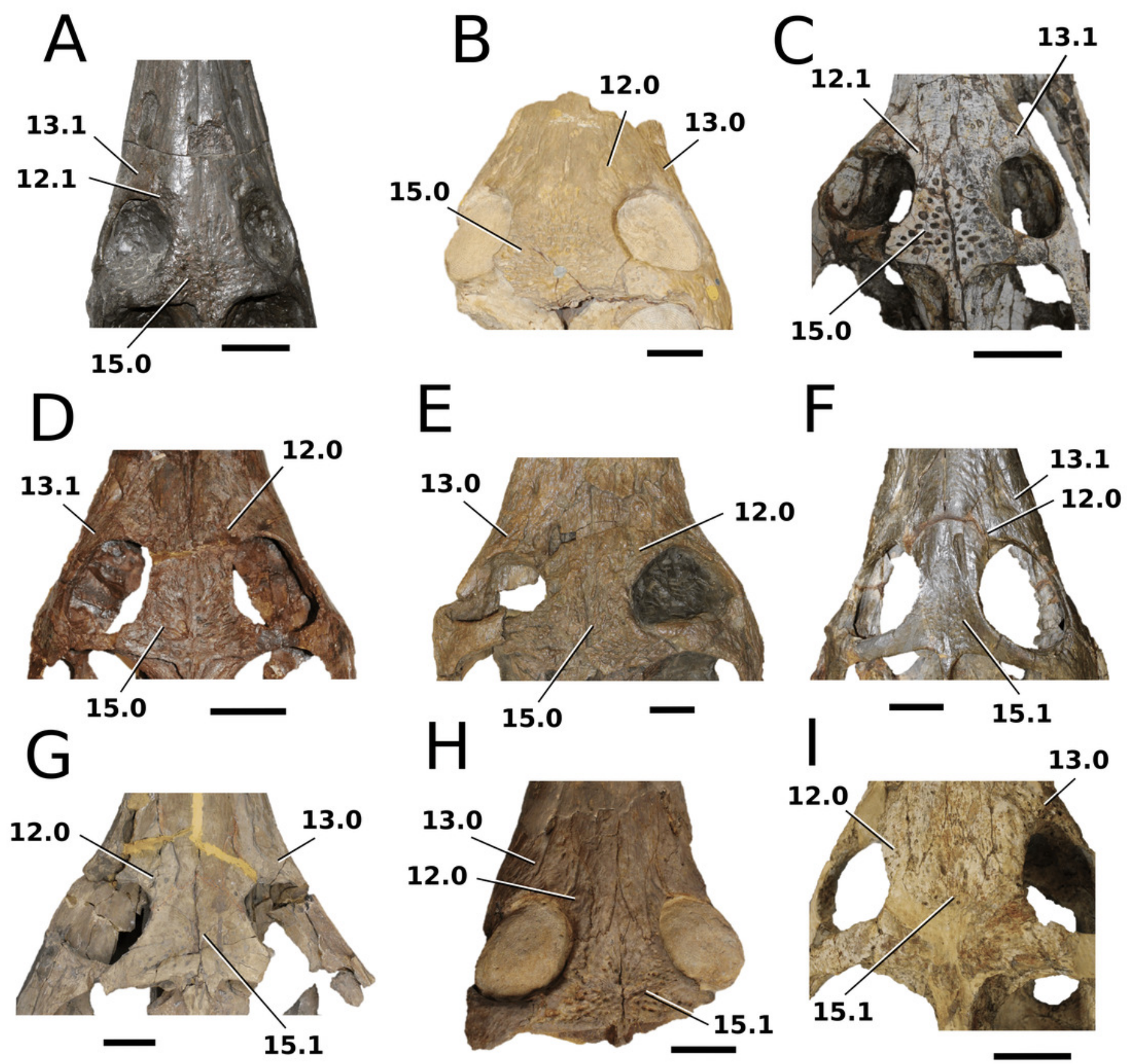


\section{Figure 30}

Comparative photographs: premaxillary anteroposterior length relative to rostrum length.

Figure 30. Comparative photographs displaying premaxillary anteroposterior length relative to rostrum length (ch. 43): (A) Macrospondylus bollensis (SMNS 81672) and (B) the Chinese teleosauroid (IVPP V 10098), as well as (C) Metriorhynchus superciliosus (LPP.M.48). Dashed lines (')') represent anteroposterior premaxillary length, while solid lines (-) represent total rostral length. Scale bars: $10 \mathrm{~cm}$. 

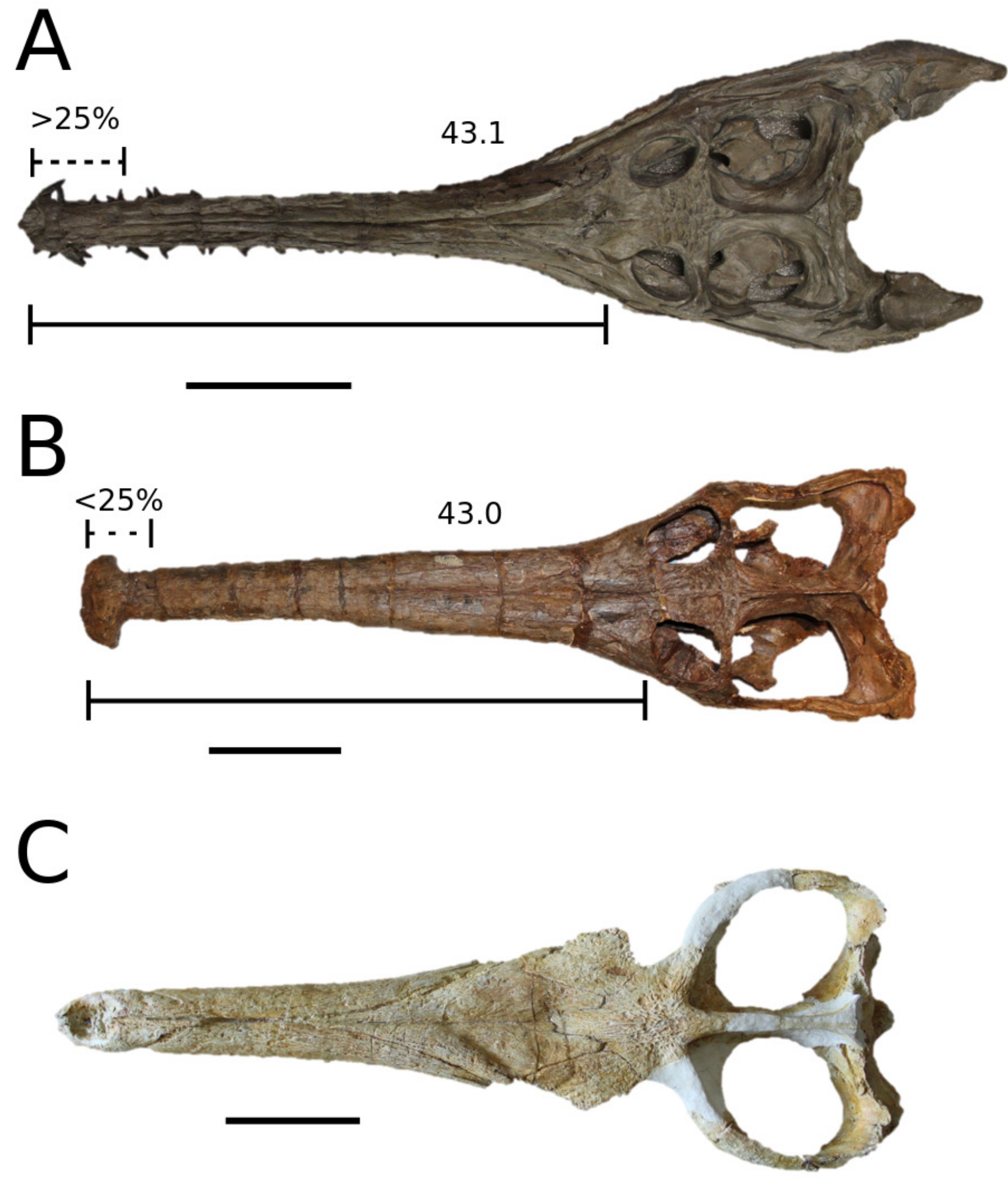


\section{Figure 31}

Comparative photographs: medial margins of the external nares, and the premaxillamaxilla suture.

Figure 31. Comparative photographs displaying medial margins of the external nares (ch. 56) and the premaxilla-maxilla suture (ch. 58): (A) Mycterosuchus nasutus (CAMSM J.1420), (B) Bathysuchus megarhinus (unnumbered LPP specimen), (C) the Chinese teleosauroid (IVPP V 10098), (D) Macrospondylus bollensis (MMG BwJ 565), (E) Deslongchampsina larteti (OUMNH J.29851), (F) Steneosaurus rostromajor (MNHN.RJN 134C-d), (G) Mystriosaurus laurillardi (NHMUK PV OR 14781), (H) Neosteneosaurus edwardsi (NHMUK PV R 2685) and (I) Charitomenosuchus leedsi (NHMUK PV R 3320). Scale bars: $3 \mathrm{~cm}$. 

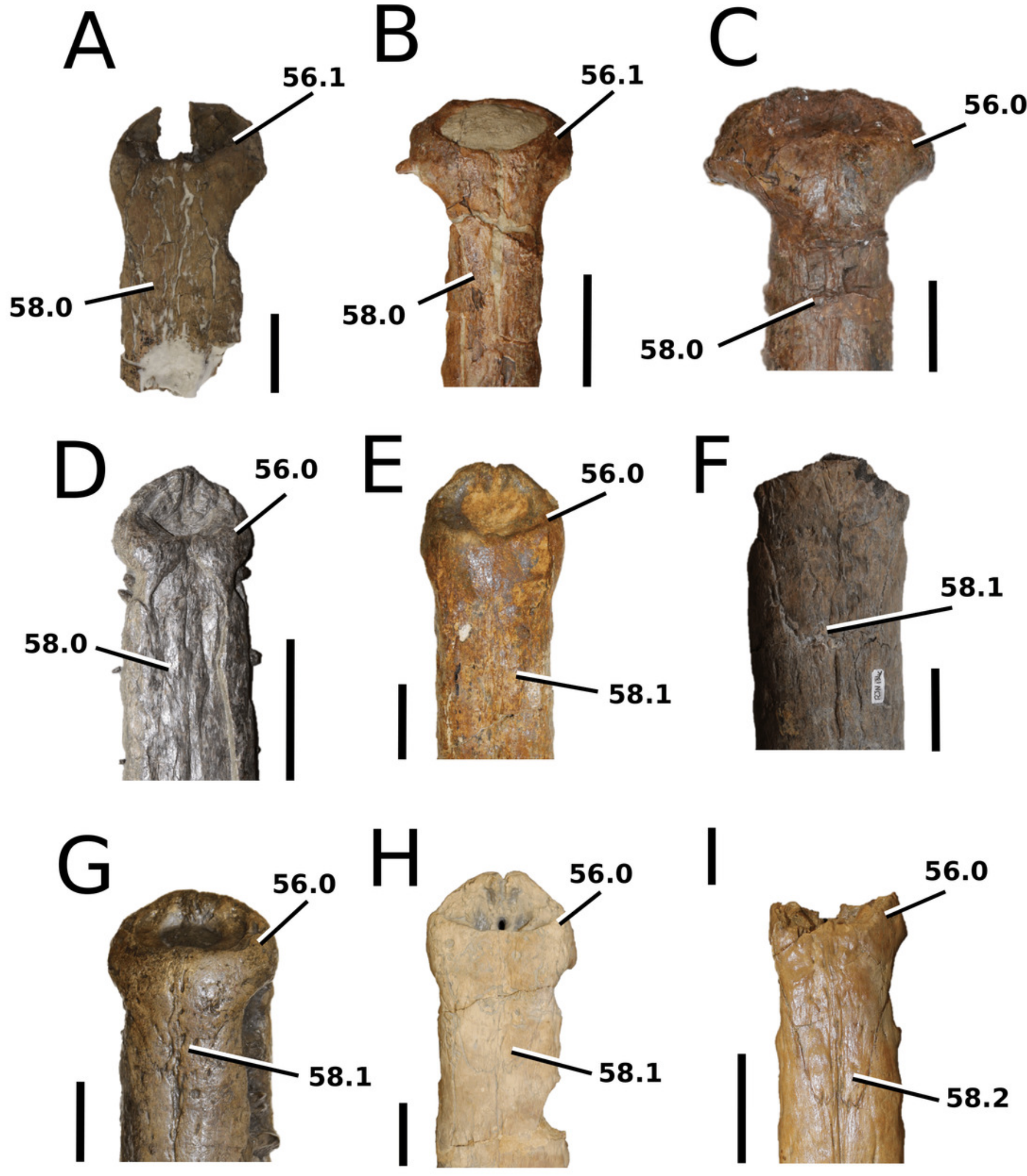


\section{Figure 32}

Comparative photographs: elongated posterior nasal processes, anteromedial frontal process, and additional anterolateral frontal projections.

Figure 32. Comparative photographs displaying the presence/absence of elongated posterior nasal processes (ch. 64), anteromedial frontal process (ch. 124) and additional anterolateral frontal projections (ch.125): (A) Indosinosuchus potamosiamensis (PRC-11), (B Mycterosuchus nasutus (NHMUK PV R 2617), (C) Macrospondylus bollensis (NHMW-1878-0047-0001), (D) Clovesuurdameredeor stephani (NHMUK PV OR 49126), (E) Charitomenosuchus leedsi (NHMUK PV R 3320), Neosteneosaurus edwardsi ((F): MNHN.RJN 118; (G) NHMUK PV R 2865), (H) Lemmysuchus obtusidens (LPP.M.21), (I) Machimosaurus buffetauti (SMNS91415) and (J) Platysuchus multiscrobiculatus (SMNS 9930). Platysuchus photograph provided by MTY. Scale bars: $4 \mathrm{~cm}$. 

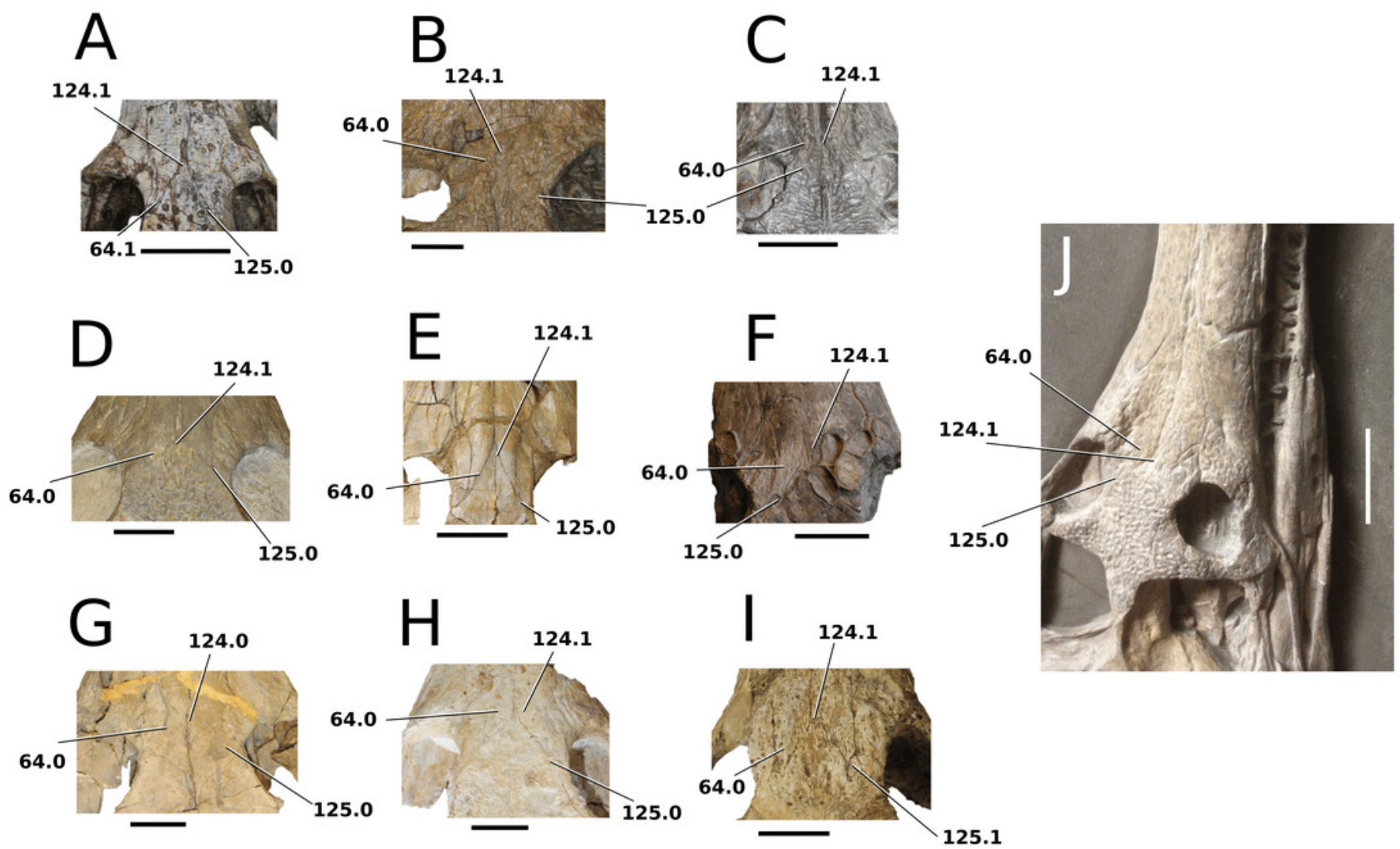


\section{Figure 33}

Comparative photographs: anterior elongation of the jugal.

Figure 33. Comparative photographs displaying the anterior elongation of the jugal (ch. 167) in (A) Plagiophthalmosuchus gracilirostris (NHMUK PV OR 14792); (B) Deslongchampsina larteti (OUMNH J.29851); (C) Charitomenosuchus leedsi (NHMUK PV R 3320); and (D) Proexochokefalos heberti (MNHN.F 1890-13). Scale bars: $5 \mathrm{~cm}$.

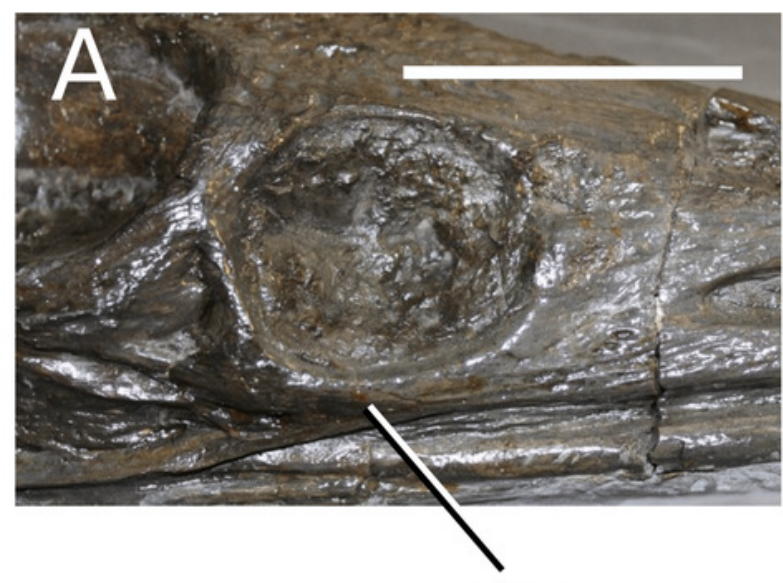

167.0

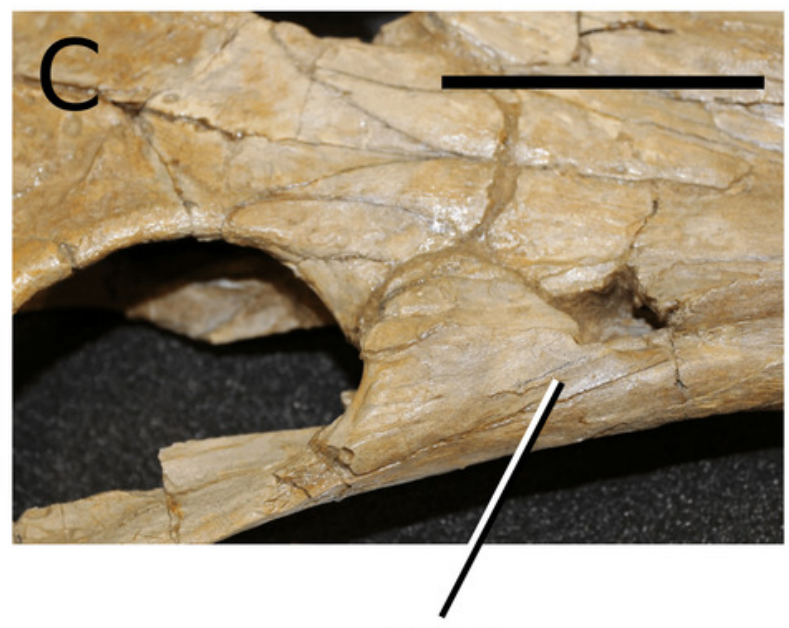

167.1

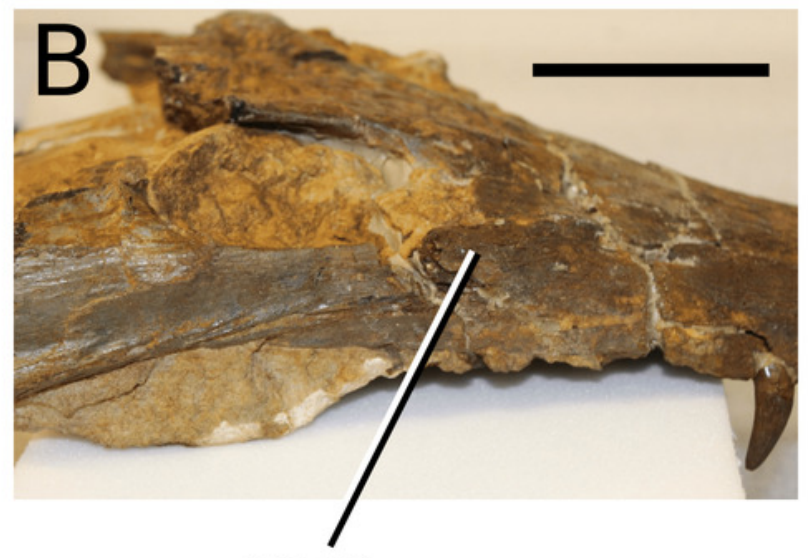

167.0

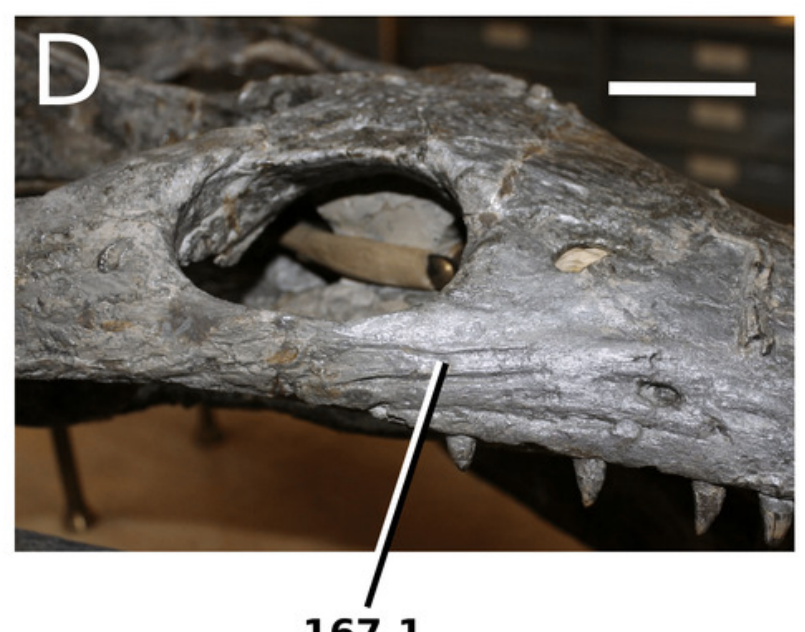

167.1 


\section{Figure 34}

Comparative photographs: premaxillary-maxillary suture in palatal view.

Figure 34. Comparative photographs displaying the premaxillary-maxillary suture in palatal view (ch. 184): (A) Teleosauroidea (Lemmysuchus obtusidens LPP.M.21) and (B) Metriorhynchoidea (Metriorhynchus supercilious LPP.M.48). Scale bars: $7 \mathrm{~cm}$. 

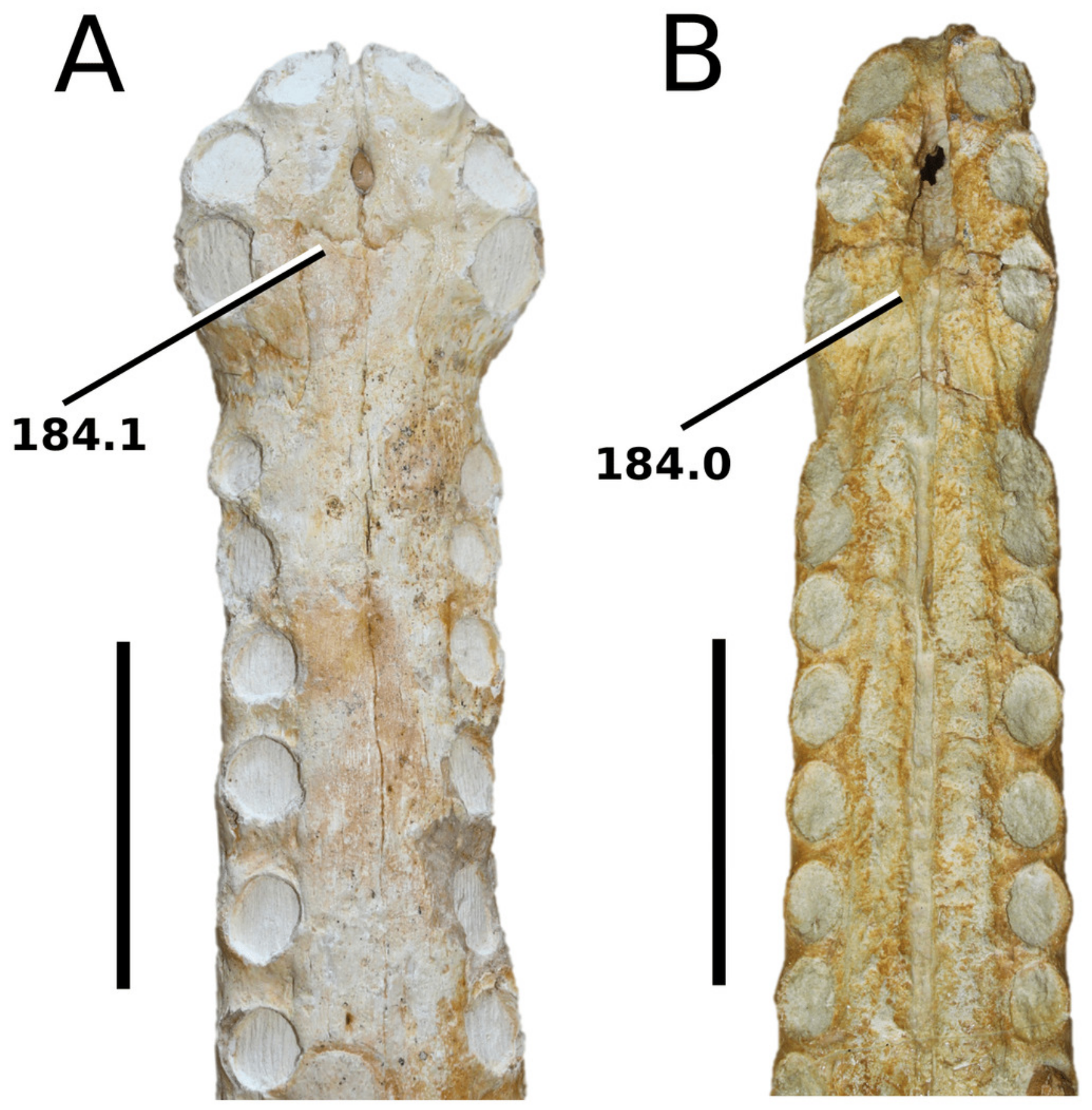


\section{Figure 35}

Comparative photographs: exoccipital and paraoccipital processes.

Figure 35. Comparative photographs displaying the exoccipital and paraoccipital processes (ch. 208): (A) Plagiophthalmosuchus gracilirostris (MNHNL TU515), (B ‘Steneosaurus' sp. (IRSNB R 0140), (C) Proexochokefalos heberti (MNHN.F 1890-13), (D) Neosteneosaurus edwardsi (PETMG R178) and (E) Machimosaurus hugii (MG 8730). Scale bars: $5 \mathrm{~cm}$.
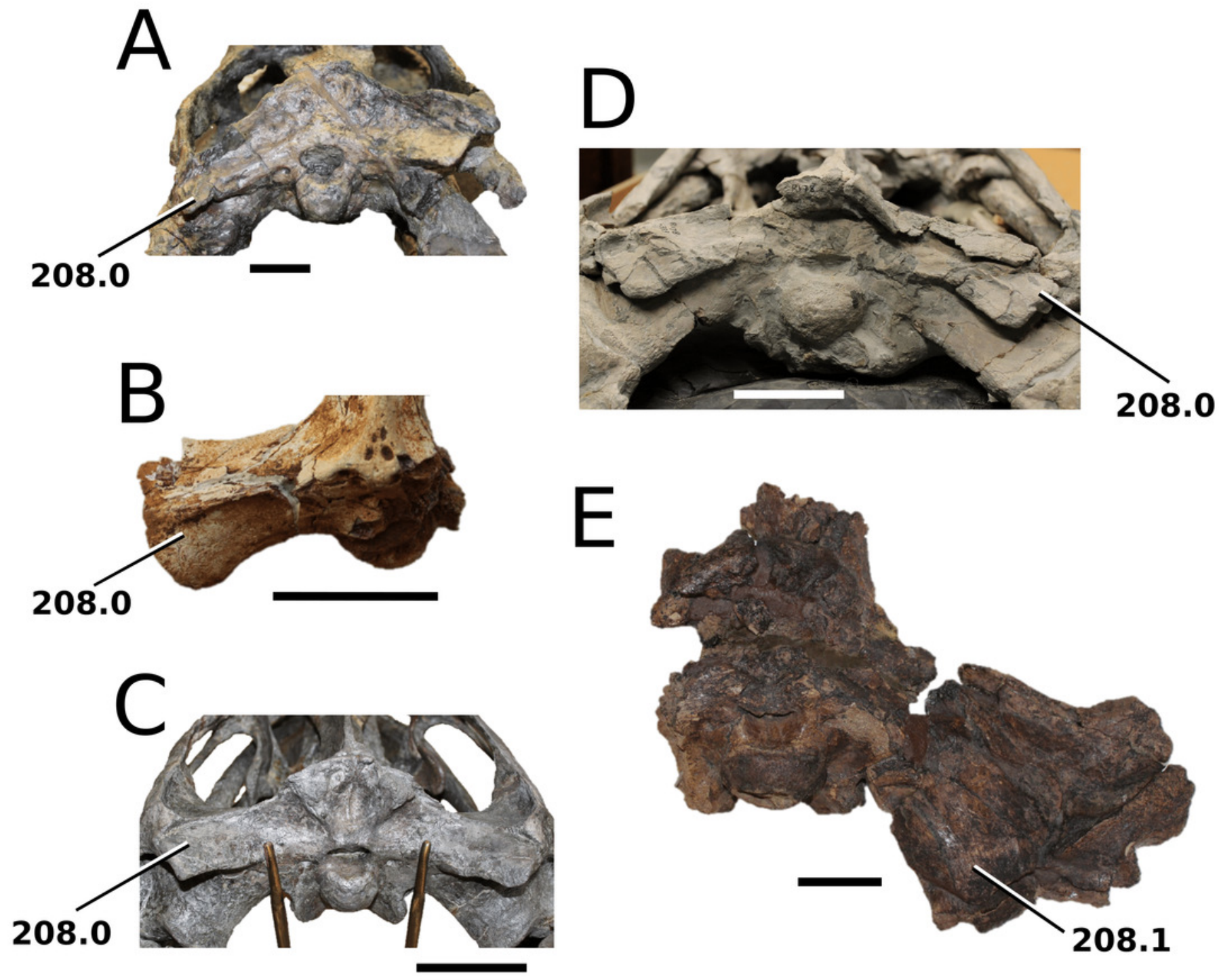


\section{Figure 36}

Comparative photographs: Meckelian groove (= canal).

Figure 36. Comparative photographs displaying the Meckelian groove (canal) (ch. 269) in (A) Mycterosuchus nasutus (NHMUK PV R 2617), (B) Macrospondylus bollensis (53422), (C)

Charitomenosuchus leedsi (NHMUK PV R 3806), (D) Steneosaurus hulkei (= Neosteneosaurus edwardsi) (NHMUK PV R 2074), (E) Yvridiosuchus boutilieri (OUMNH J.1404), (F) Lemmysuchus obtusidens (LPP.M.21), and (G) Machimosaurus mosae (Young et al., 2014). Scale bars: $3 \mathrm{~cm}$.

A

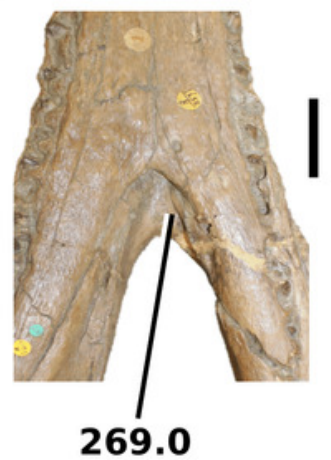

B

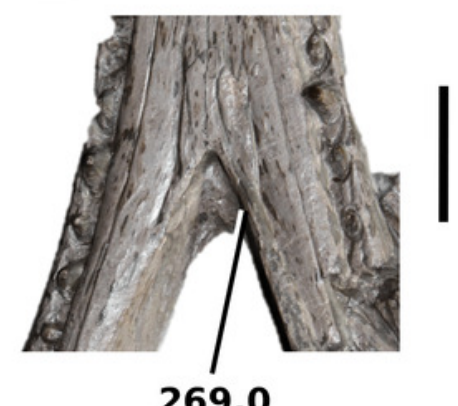

269.0
D

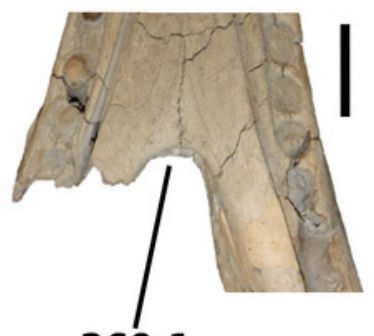

269.1
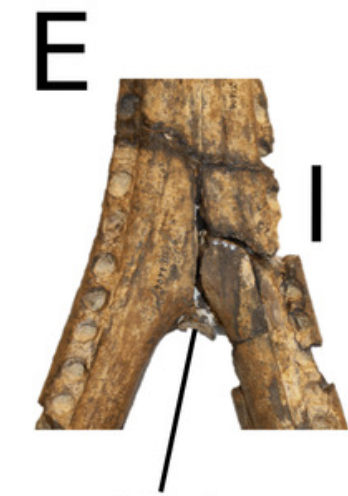

269.1
E

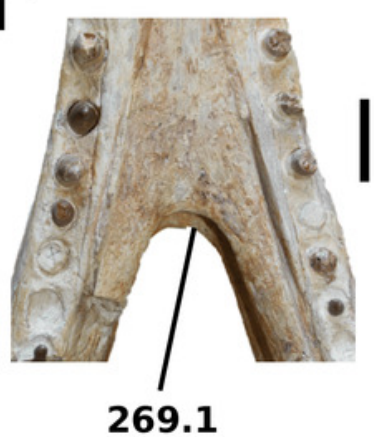

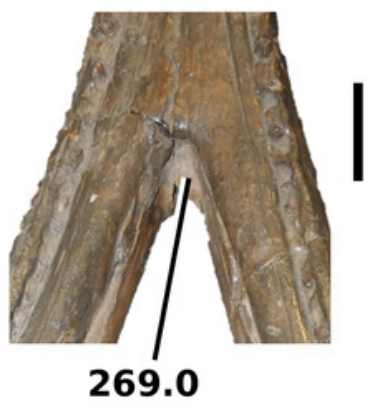

0

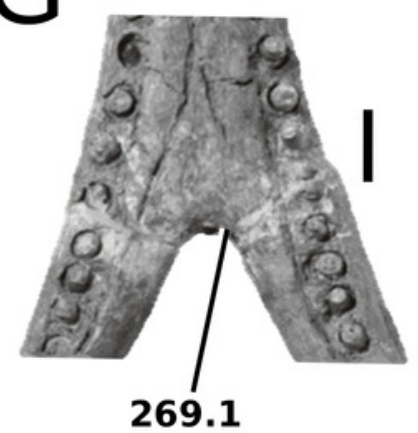




\section{Figure 37}

Comparative photographs: curvature of the retroarticular process.

Figure 37. Comparative photographs displaying the curvature of the retroarticular process (ch. 270) (in lateral view). (A) Plagiophthalmosuchus gracilirostris (MNHNL TU515), (B) Mystriosaurus laurillardi (NHMUK PV OR 14781), (C) Mycterosuchus nasutus (NHMUK PV R 2617), (D) Charitomenosuchus leedsi (NHMUK PV R 3806), (E) Macrospondylus bollensis (SMNS 58876), (F) Proexochokefalos heberti (MNHN.F 1890-13), (G) Machimosaurus buffetauti (SMNS 91415) and (H) Yvridiosuchus boutilieri (OUMNH J.29850). Scale bars: $15 \mathrm{~cm}$ (B, E-F) and $5 \mathrm{~cm}(\mathrm{~A}, \mathrm{C}-\mathrm{D}, \mathrm{G}-\mathrm{H})$. 

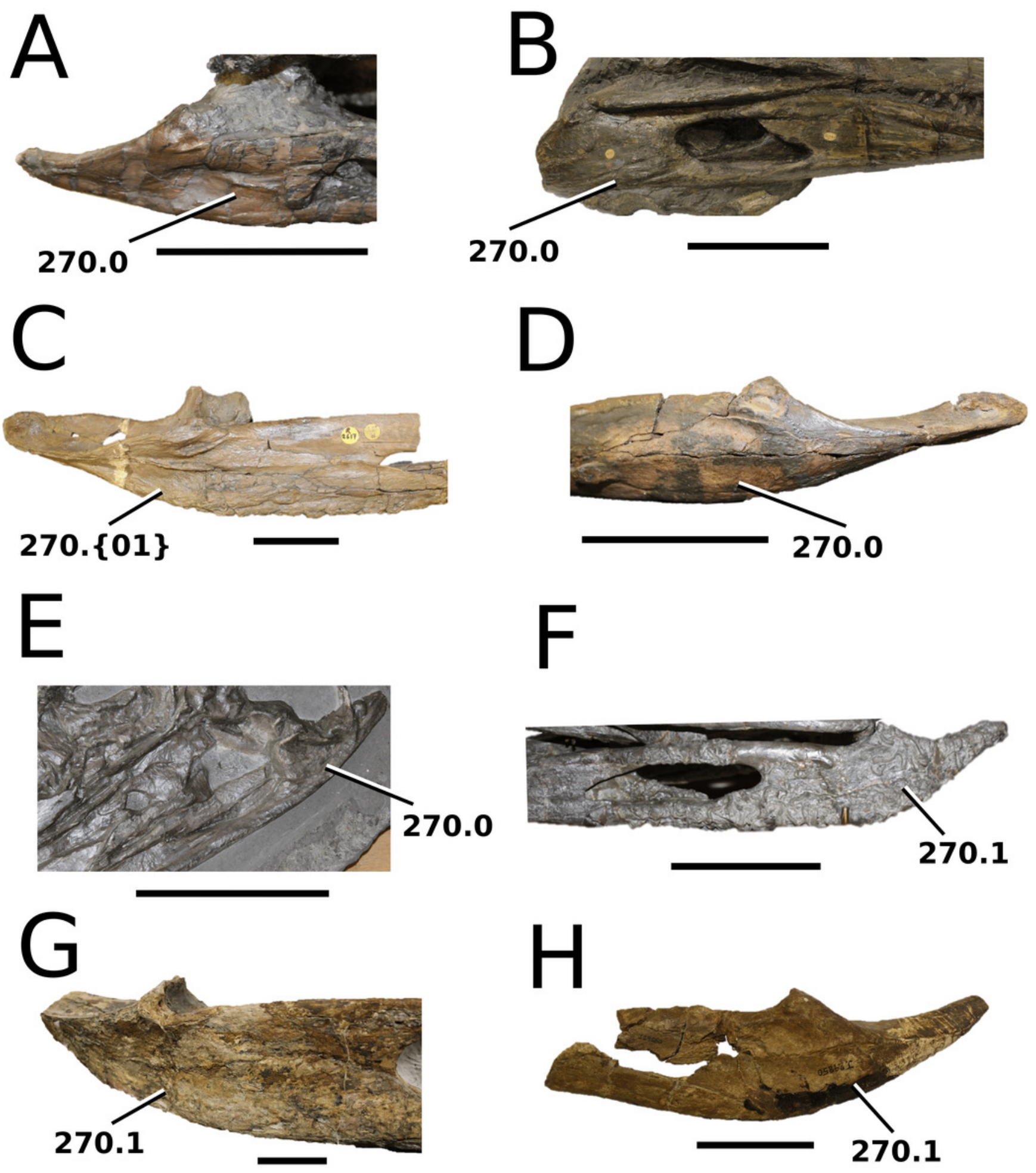


\section{Figure 38}

Comparative photographs: reception pits.

Figure 38. Comparative photographs displaying the reception pits (in right lateral view) (ch. 291). (A) Plagiophthalmosuchus gracilirostris (NHMUK PV OR 15500), (B) Mystriosaurus laurillardi (NHMUK PV OR 14781), (C) Proexochokefalos heberti (MNHN.F 1890-13) and (D) Lemmysuchus obtusidens (LPP.M.21). Scale bars: $17 \mathrm{~cm}$.
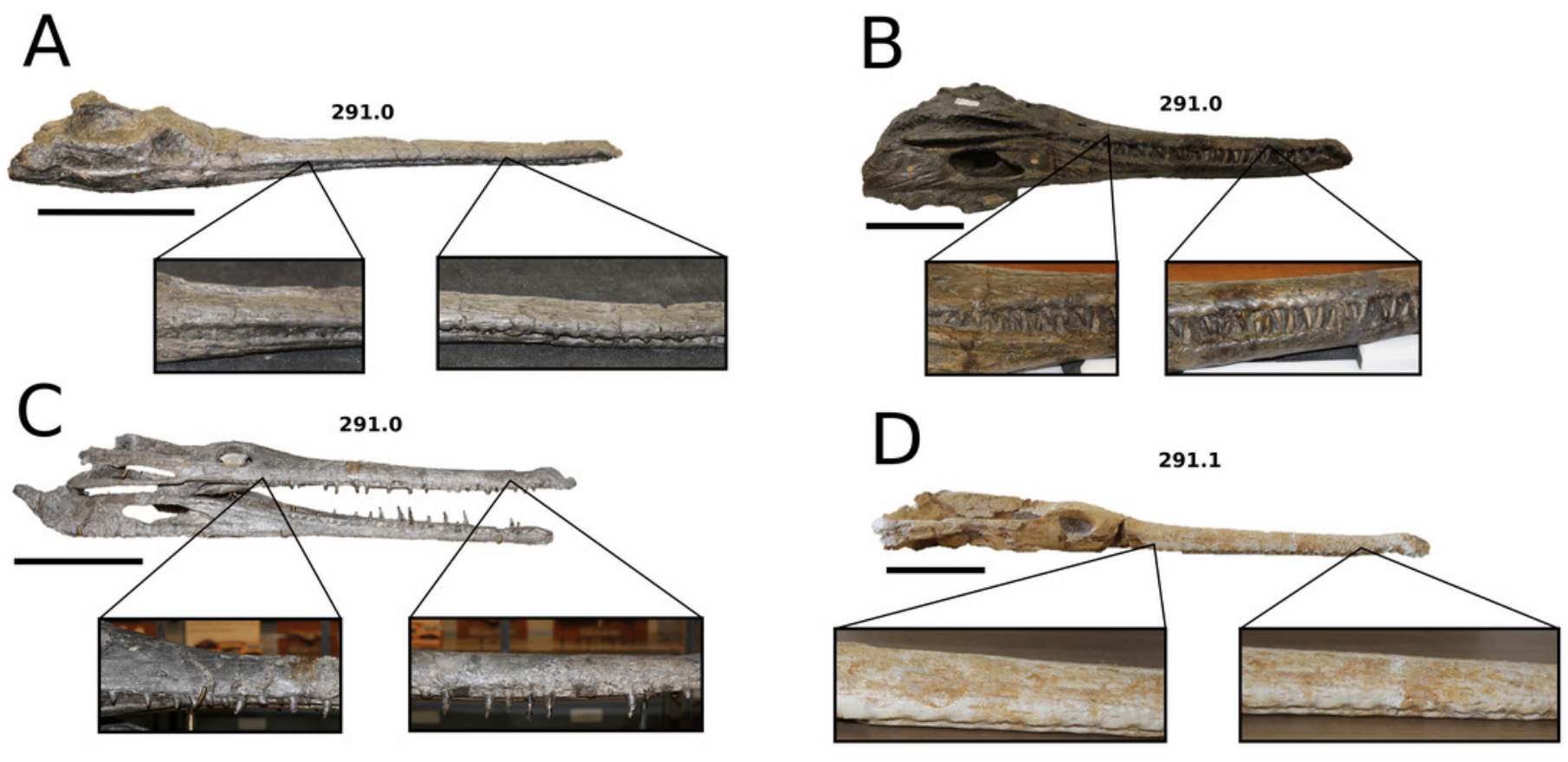


\section{Figure 39}

Comparative photographs: characteristic features of the premaxillary alveoli.

Figure 39. Comparative photographs displaying characteristic features of the premaxillary alveoli (ch. 292 to 297), in: (A) the Chinese teleosauroid (IVPP V 10098), (B) Bathysuchus megarhinus (DORCM G.05067i; Foffa et al., 2019), (C) Indosinosuchus potamosiamensis (PRC-11), (D) Platysuchus multiscrobiculatus (MNHNL. TU895), (E) Charitomenosuchus leedsi (NHMUK PV R 3806), (F) Mystriosaurus sp. (SNHM-IG-008-R), (G) Yvridiosuchus boutilieri (OUMNH J.1401) and (H) Lemmysuchus obtusidens (LPP.M.21). Note that character 294 and 295 are inapplicable for the Chinese teleosauroid (IVPP V 10098). Scale bars: $3 \mathrm{~cm}$.
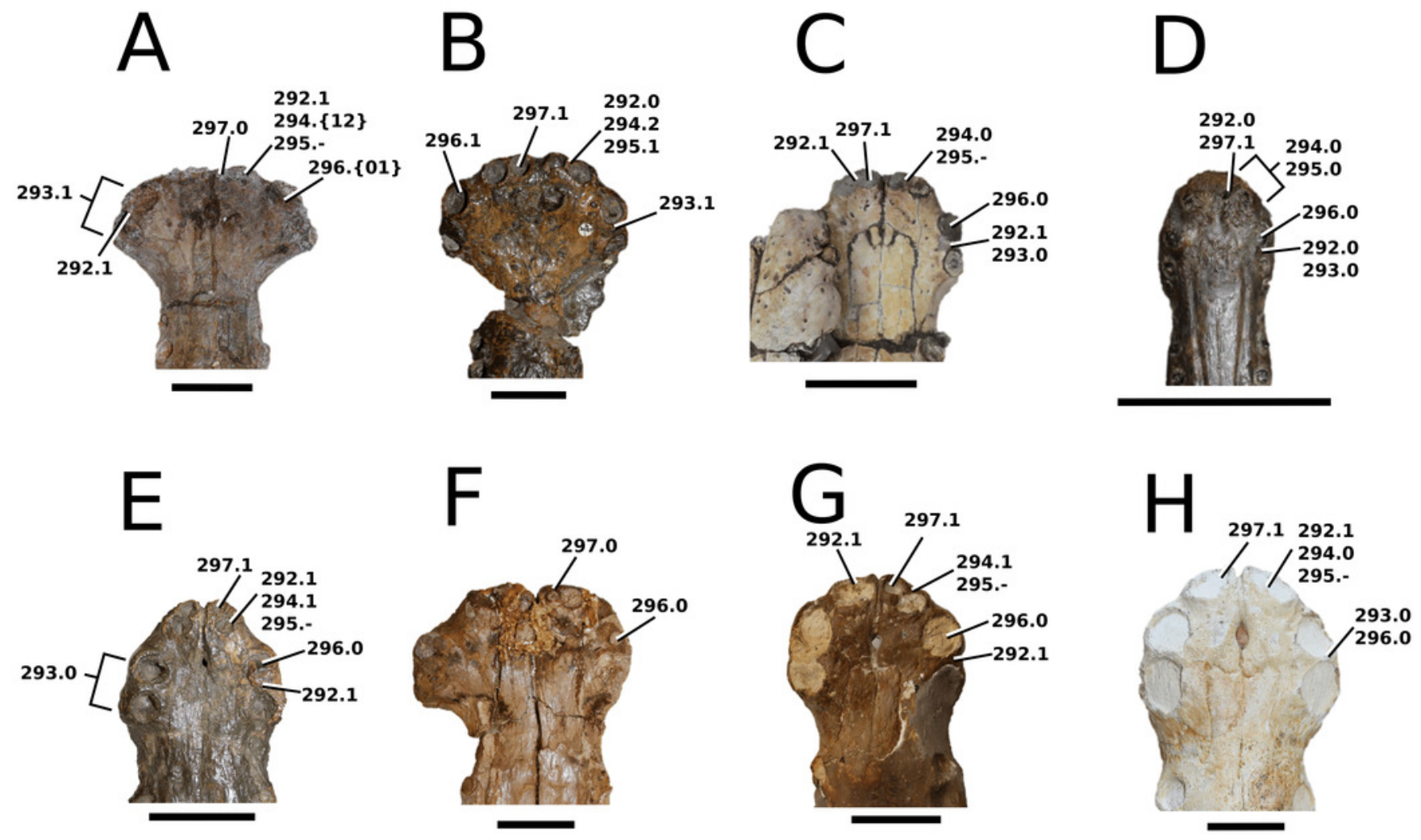


\section{Figure 40}

Comparative photographs: teleosauroid teeth.

Figure 40. Comparative photographs of teleosauroid teeth, highlighting the carinae (ch.

339-340), apices (ch. 327) and anastomosing pattern (ch. 358): (A) Bathysuchus megarhinus

(DORCM G.05067iv; Foffa et al., 2019), (B) Sericodon jugleri (NRM-PZ 2337), (C)

Proexochokefalos heberti (MNHN.F 1890-13), (D) Deslongchampsina larteti (OUMNH J.29851),

(F) Neosteneosaurus edwardsi (NHMUK PV R 2865), (F) Machimosaurini indeterminate (GPIT-

RE-301), (G) Yvridiosuchus boutilieri (OUMNH J.29850), and (H) Machimosaurus hugii (MG 25). Scale bars: $3 \mathrm{~cm}(A-B, E)$ and $1 \mathrm{~cm}(C-D, F-H)$.
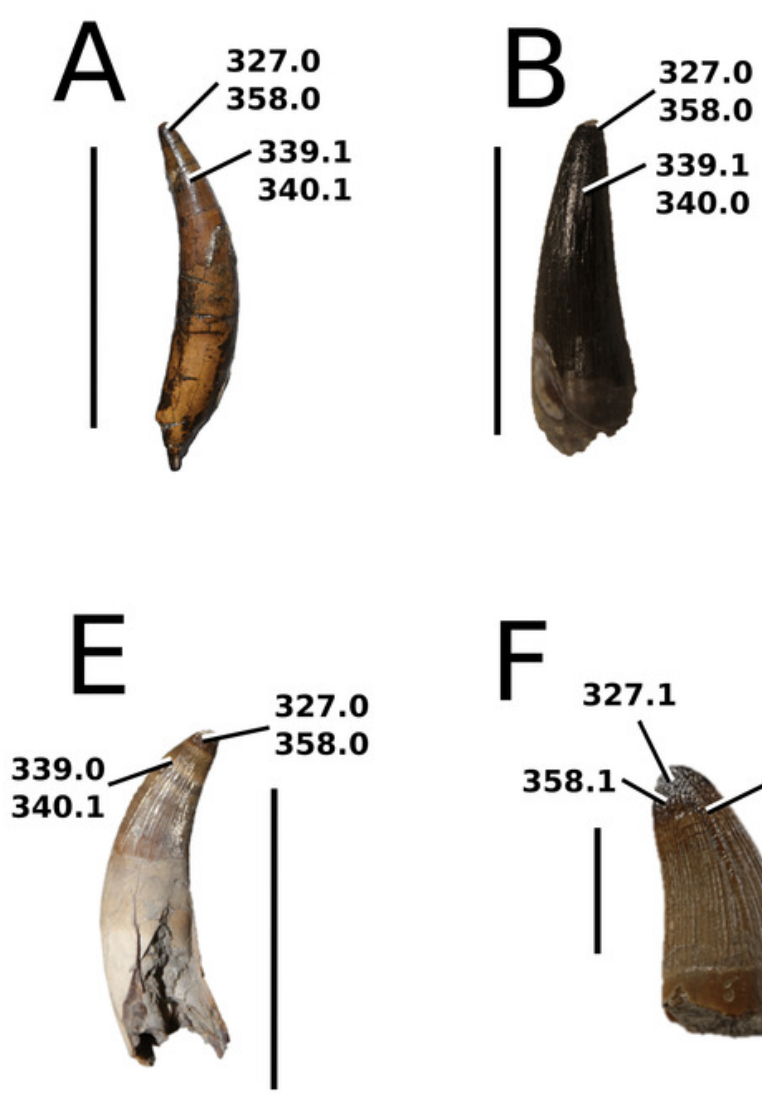
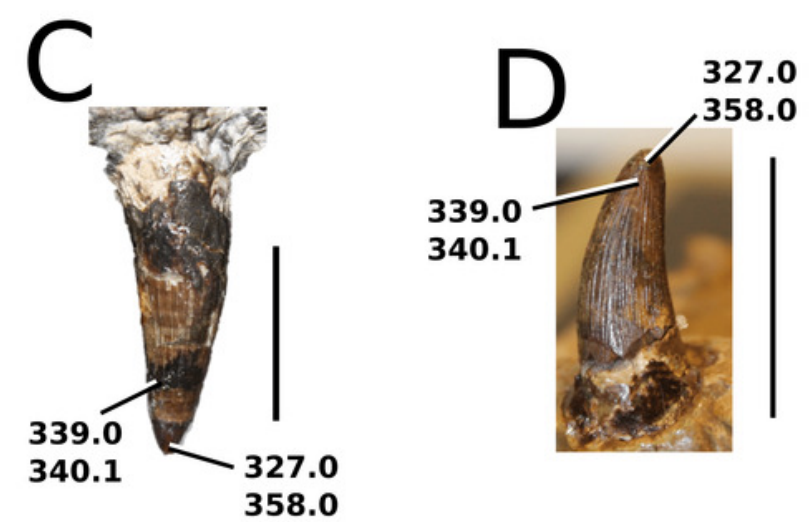

358.0
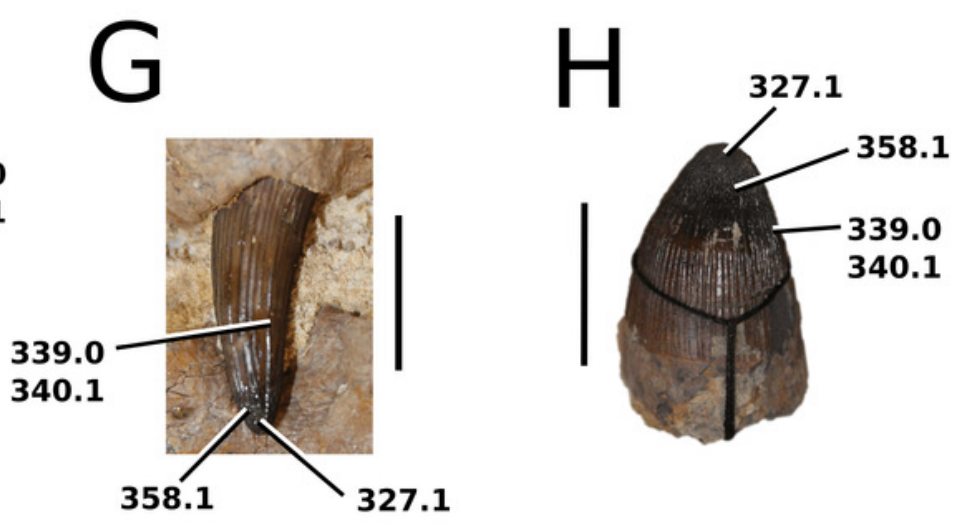


\section{Figure 41}

Comparative photographs: teleosauroid cervical ribs.

Figure 41. Comparative photographs of teleosauroid cervical ribs (ch. 394): (A)

Macrospondylus bollensis (SMNS 51984), (B) Mycterosuchus nasutus (NHMUK PV R 2617), (C) Neosteneosaurus edwardsi (NHMUK PV R 3701) and (D) Lemmysuchus obtusidens (NHMUK PV R 3168). Scale bars: $3 \mathrm{~cm}$.
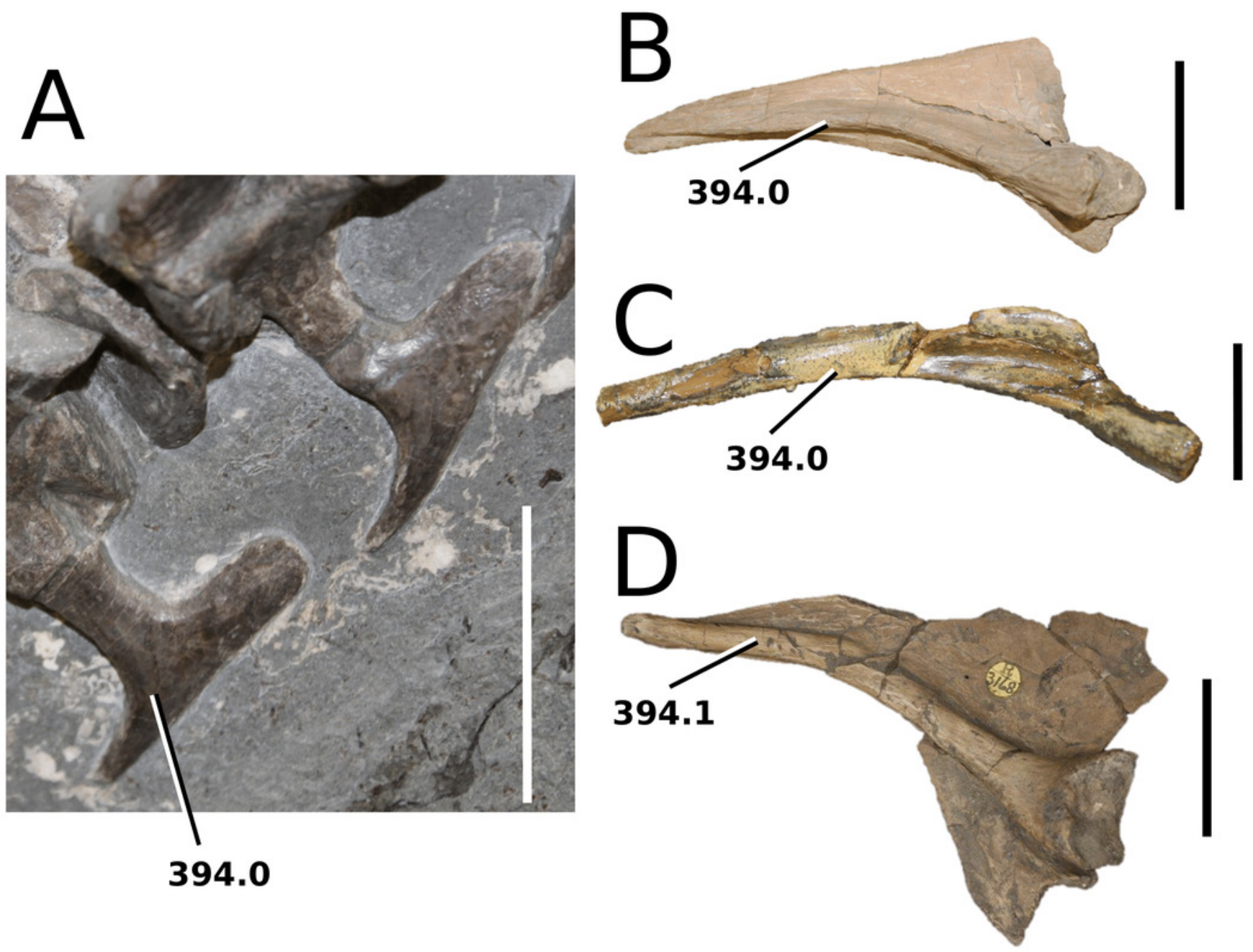


\section{Figure 42}

Comparative photographs: teleosauroid dorsal ribs.

Figure 42. Comparative photographs of teleosauroid dorsal ribs (ch. 395 and 396) (from the middle of the ribcage); (A) Charitomenosuchus leedsi (NHMUK PV R 3806), (B)

Neosteneosaurus edwardsi (PETMG R178), (C) Lemmysuchus obtusidens (NHMUK PV R 3168) and (D) Macrospondylus bollensis (SMNS 52034). Scale bars: $3 \mathrm{~cm}$.

A

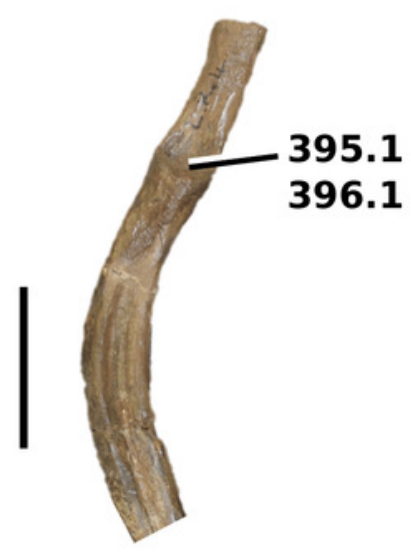

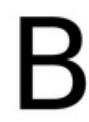

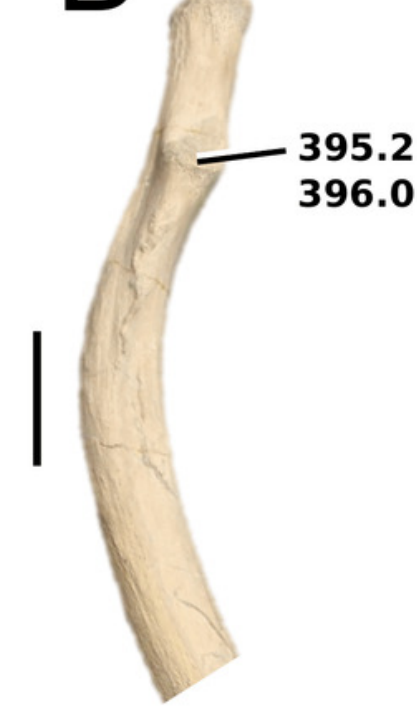

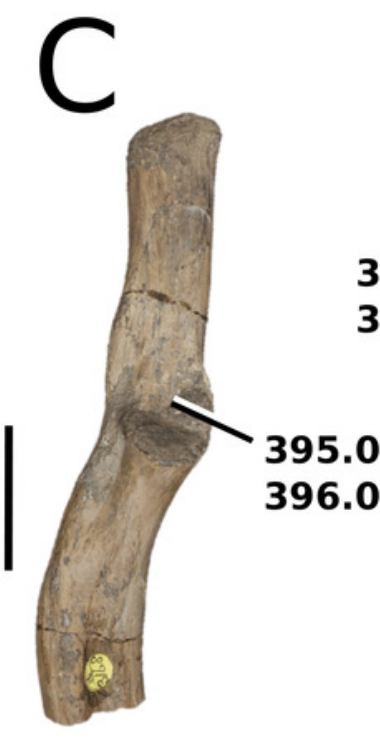

395.0

396.1

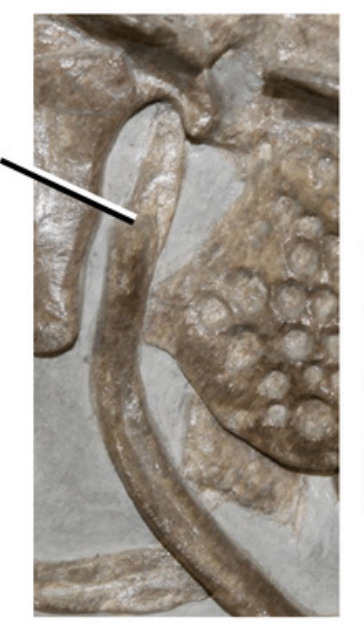




\section{Figure 43}

Comparative photographs: teleosauroid sacral vertebrae.

Figure 43. Comparative photographs of teleosauroid sacral vertebrae, with special attention to the number (ch. 379) and flange of the second sacral (ch. 398): (A) Charitomenosuchus leedsi (NHMUK PV R 3806), (B) Lemmysuchus obtusidens (NHMUK PV R 3168), (C) Mycterosuchus nasutus (NHMUK PV R 2617) and (D) Macrospondylus bollensis (GPITRE-9427). 

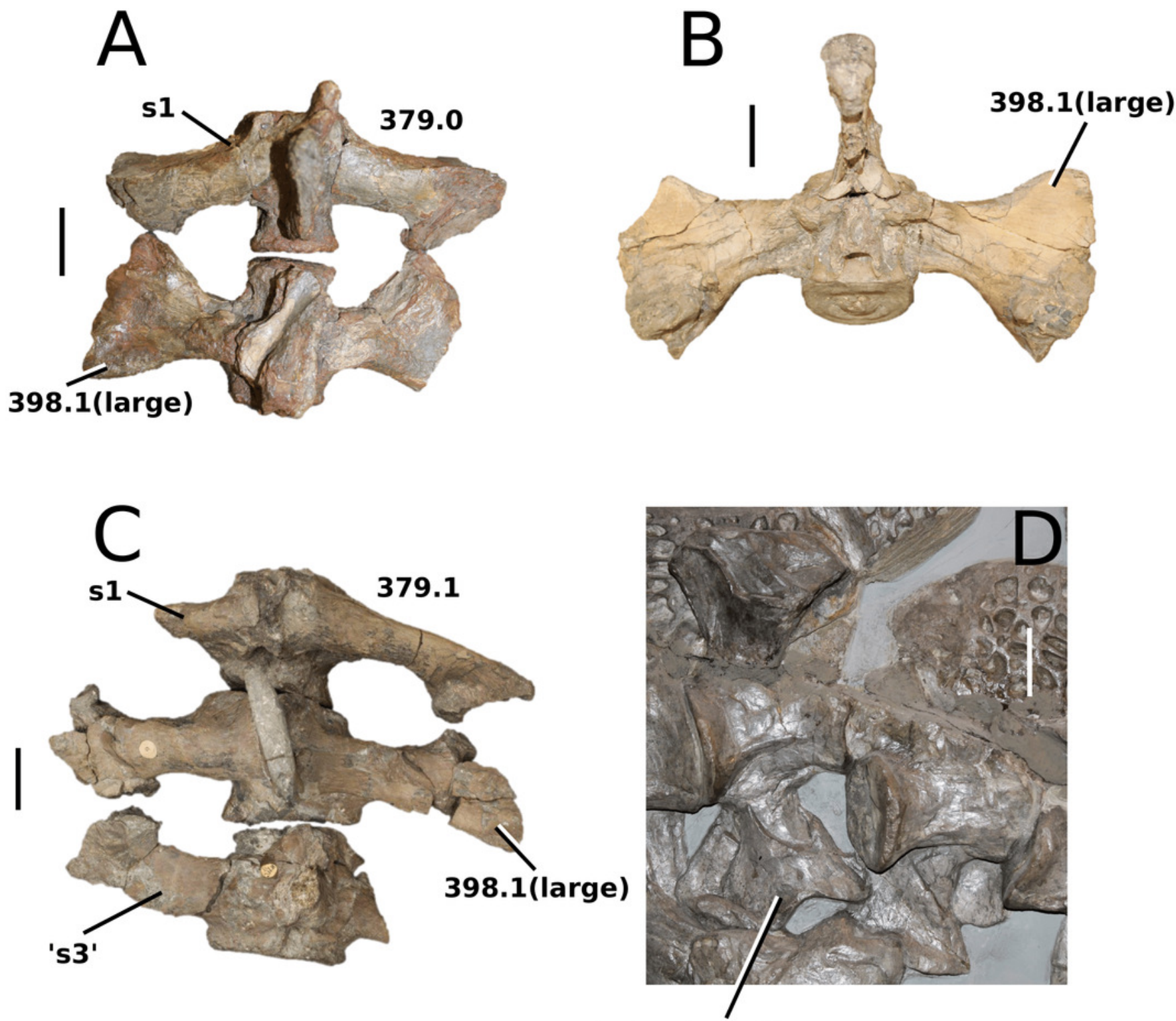

398.1(small) 


\section{Figure 44}

Comparative photographs: teleosauroid ulnae and radiae.

Figure 44. Comparative photographs of teleosauroid ulnae and radiae, with special attention to relative size (ch. 417) and proximal ulna (ch. 420): (A) Neosteneosaurus edwardsi (PETMG R178) i. ulna and ii. radius; (B) Mycterosuchus nasutus (NHMUK PV R 2617) i. ulna and ii. radius; (C) Charitomenosuchus leedsi (NHMUK PV R 3806) i. ulna and ii. radius; and (D) Macrospondylus bollensis (SMNS 53422) i. ulna and ii. radius. Scale bars: $3 \mathrm{~cm}$.

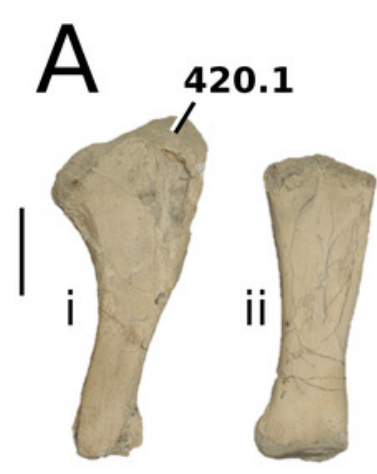

417.0

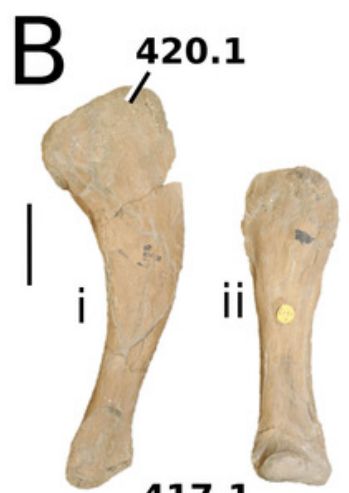

417.1

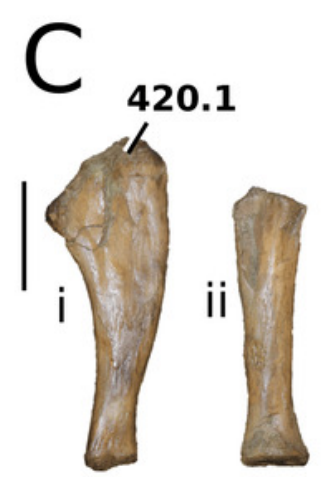

417.0

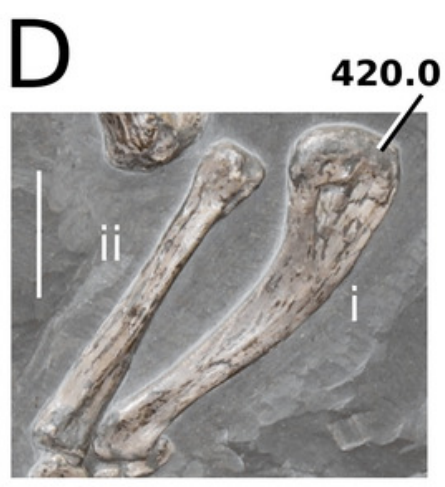

417.0 


\section{Figure 45}

Comparative photographs: teleosauroid pubes.

Figure 45. Comparative photographs of teleosauroid pubes, highlighting the pubic blade (ch. 430) and elongation (ch. 431): (A) Mycterosuchus nasutus (NHMUK PV R 2617), (B)

Charitomenosuchus leedsi (NHMUK PV R 3806), (C) Neosteneosaurus edwardsi (PETMG R178) and (D) Macrospondylus bollensis (SMNS 51957). Scale bars: $3 \mathrm{~cm}$.
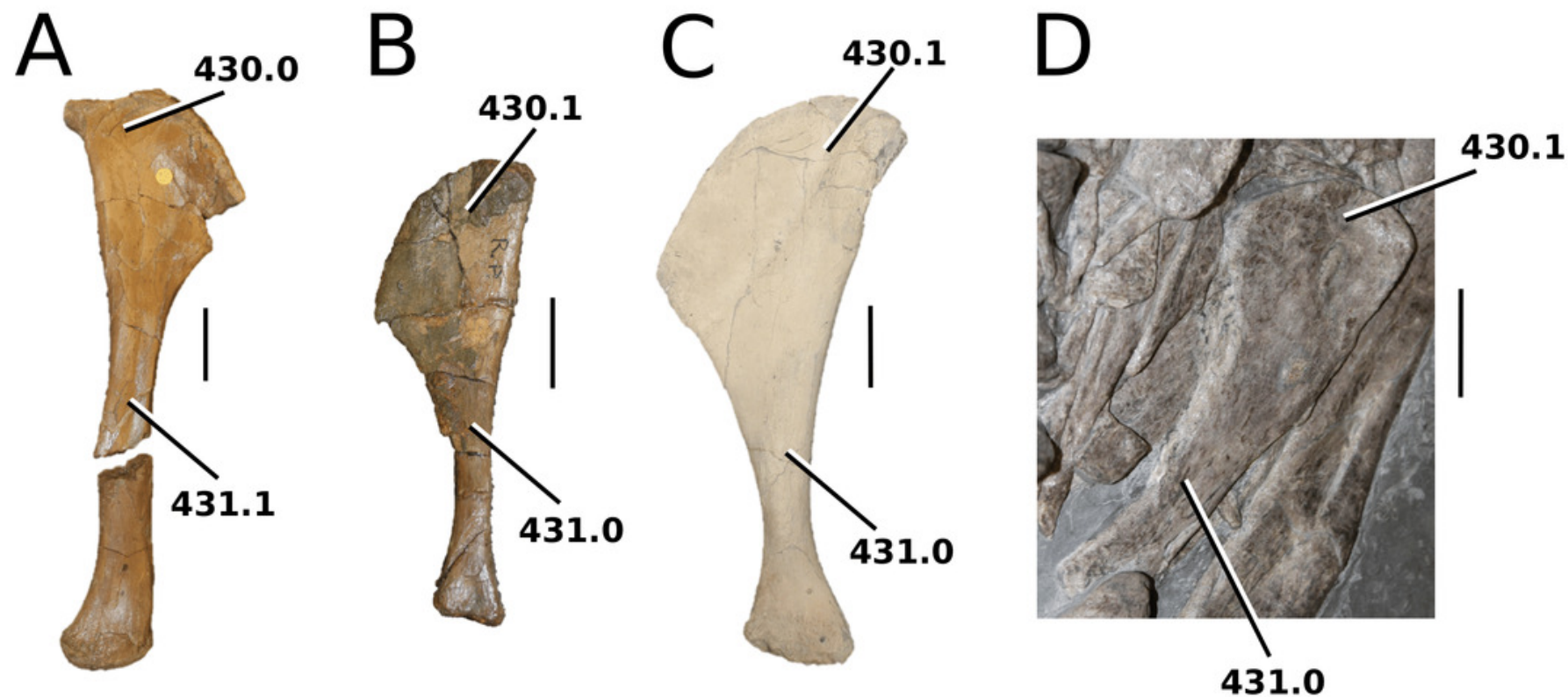


\section{Figure 46}

Comparative photographs: teleosauroid ilia.

Figure 46. Comparative photographs of teleosauroid ilia with attention to the anterior process (ch. 434), supraacetabular crest (ch. 438) and postacetabular process (ch. 440): (A)

Charitomenosuchus leedsi (NHMUK PV R 3806), (B) Macrospondylus bollensis (SMNS 18672), (C) Neosteneosaurus edwardsi (PETMG R178) and (D) Lemmysuchus obtusidens (NHMUK PV R 3168). Scale bars: $5 \mathrm{~cm}$.
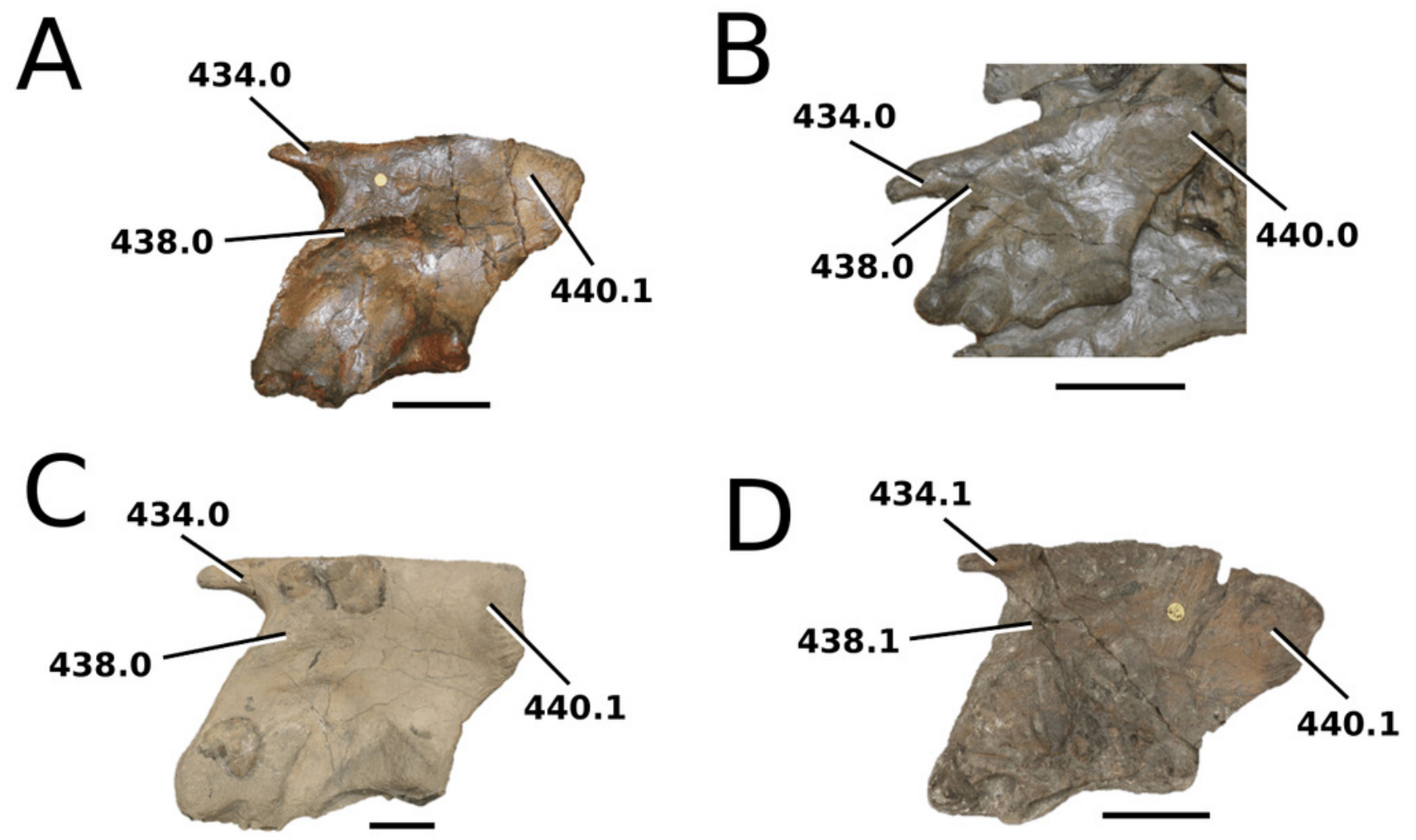


\section{Figure 47}

Comparative photographs: teleosauroid ischia.

Figure 47. Comparative photographs of teleosauroid ischia with emphasis on the ischial blade (ch. 449): (A) Platysuchus multiscrobiculatus (SMNS 9930), (B) Teleosaurus sp. (NHMUK PV 238), (C) Neosteneosaurus edwardsi (NHMUK PV R 3898), (D) Macrospondylus bollensis (SMNS 58876), (E) Aeolodon priscus (MNHN.F.CNJ 78), (F) Lemmysuchus obtusidens (NHMUK PV R 3168) and (G) Machimosaurus mosae (IRSNB cast). Scale bars: $3 \mathrm{~cm}$, (H) not to scale. 


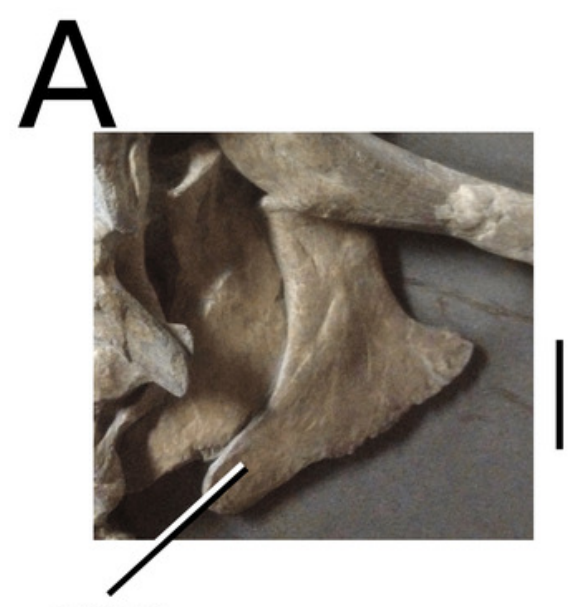

449.0
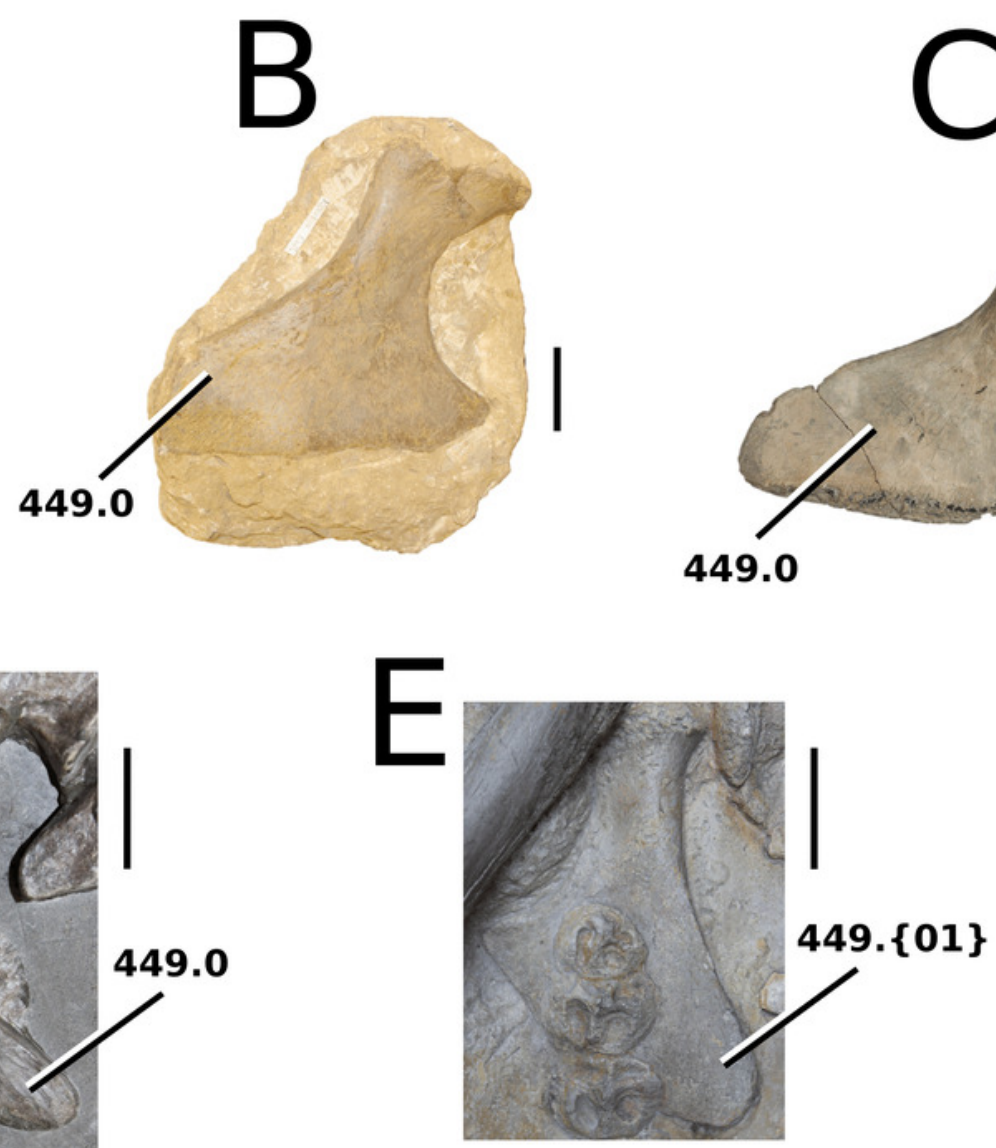
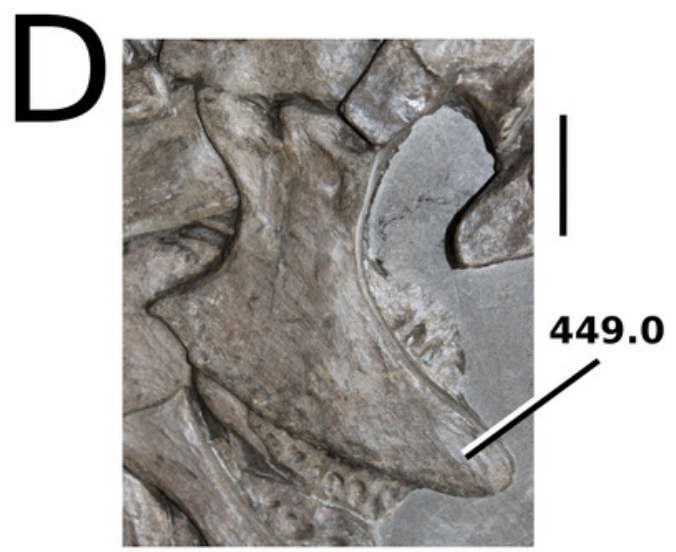

F

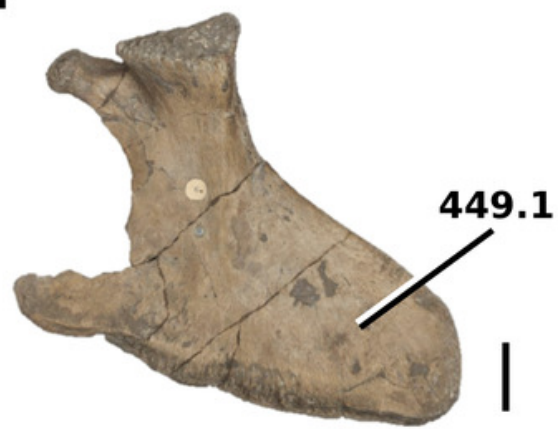

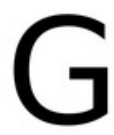

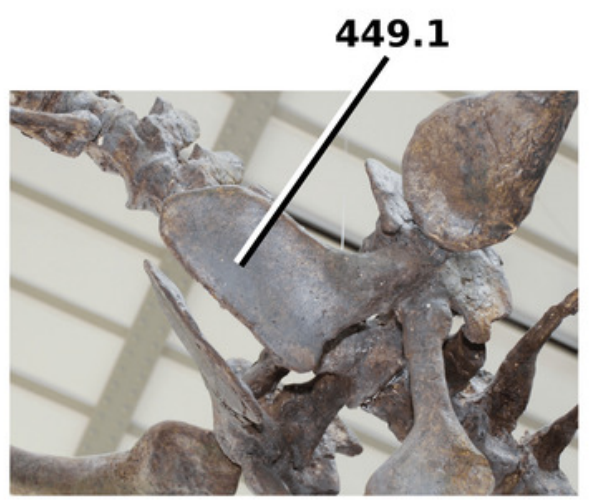




\section{Figure 48}

Comparative photographs: teleosauroid femora.

Figure 48. Comparative photographs of teleosauroid femora (ch. 456 and 459):

Mycterosuchus nasutus (NHMUK PV R 2617) [(A) femoral head dorsal view; (B) femoral condyles posterior view], Neosteneosaurus edwardsi (PETMG R178) [(C) femoral head dorsal view; (D) femoral condyles posterior view] and Macrospondylus bollensis (SMNS 51555) ((E) femoral condyles posterior view). Scale bars: $3 \mathrm{~cm}$, (E) not to scale.
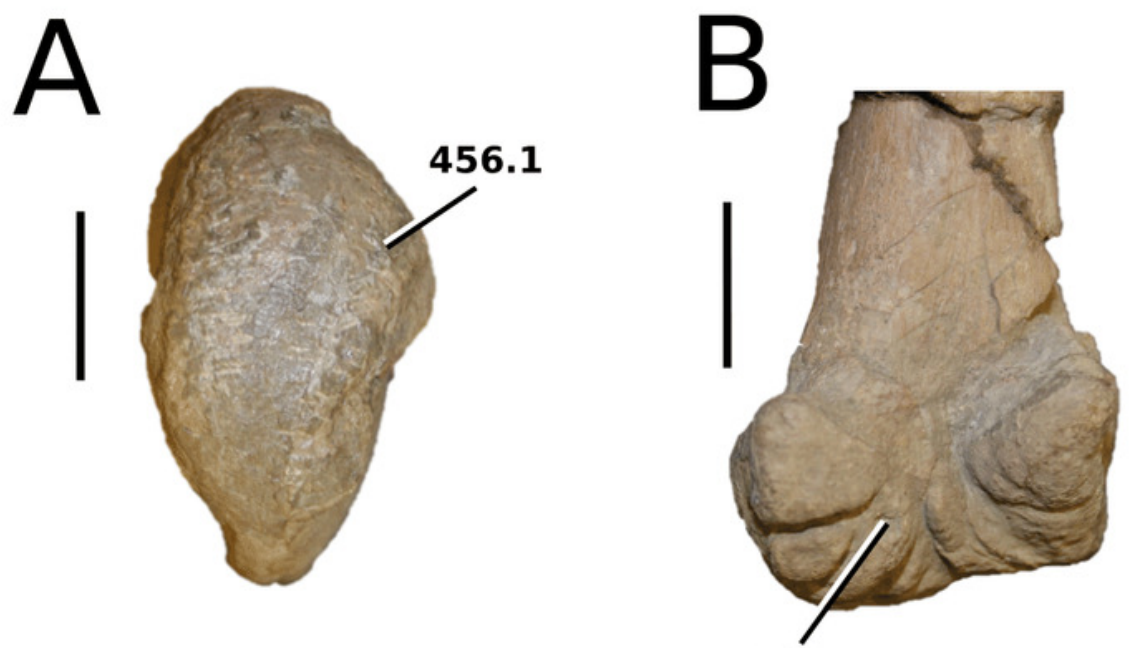

459.1
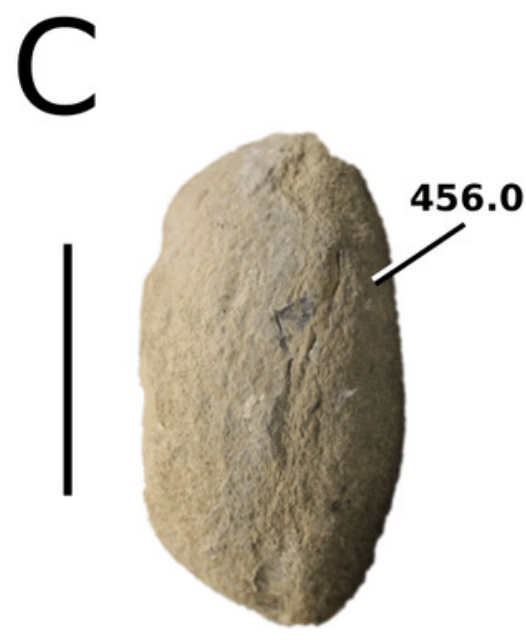
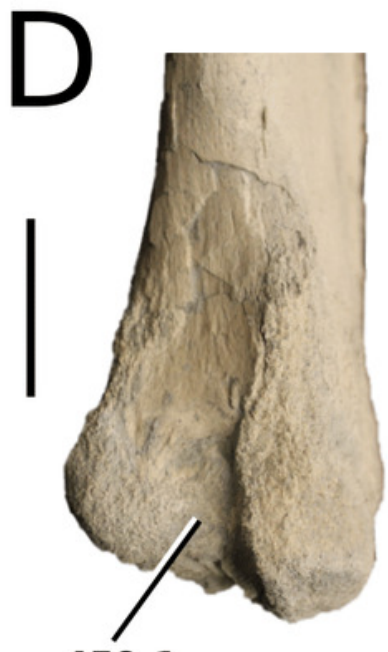

459.1

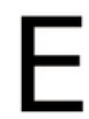

459.0 


\section{Figure 49}

Comparative photographs: teleosauroid tibiae.

Figure 49. Comparative photographs of teleosauroid tibiae, focusing on the tibal tuberosity (ch. 464): (A) Charitomenosuchus leedsi (NHMUK PV R 3806), (B) Neosteneosaurus edwardsi (PETMG R178) and (C) Lemmysuchus obtusidens (NHMUK PV R 3168). Scale bars: $3 \mathrm{~cm}$.
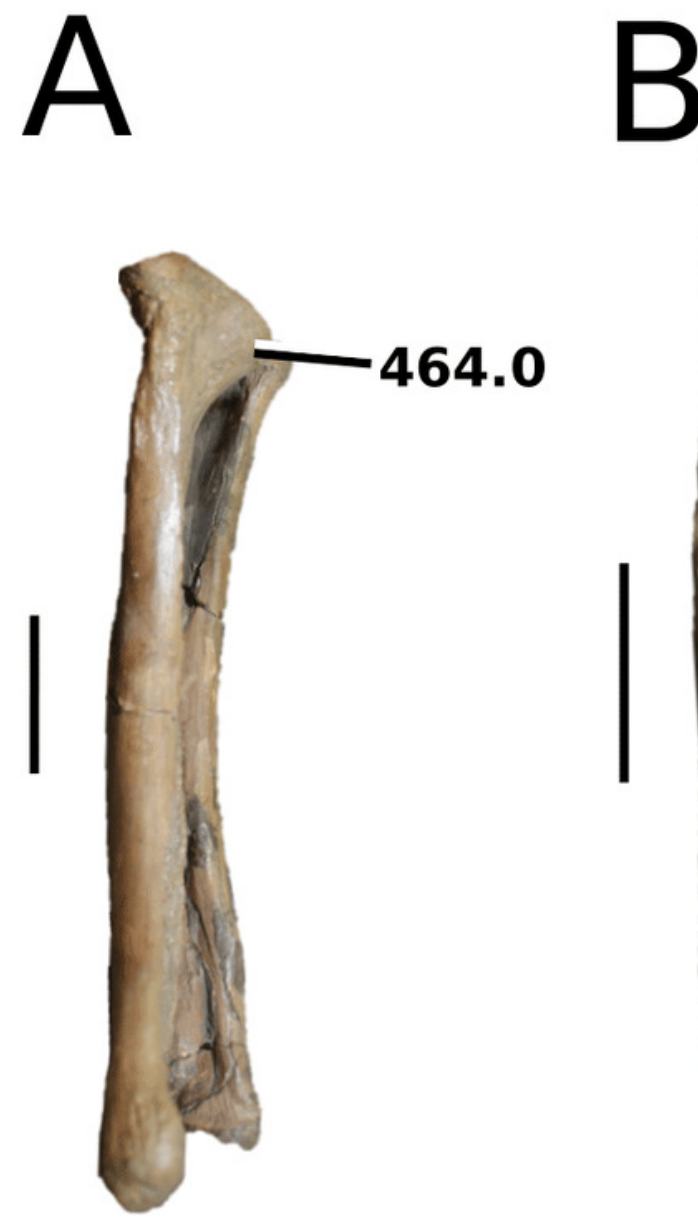

464.0
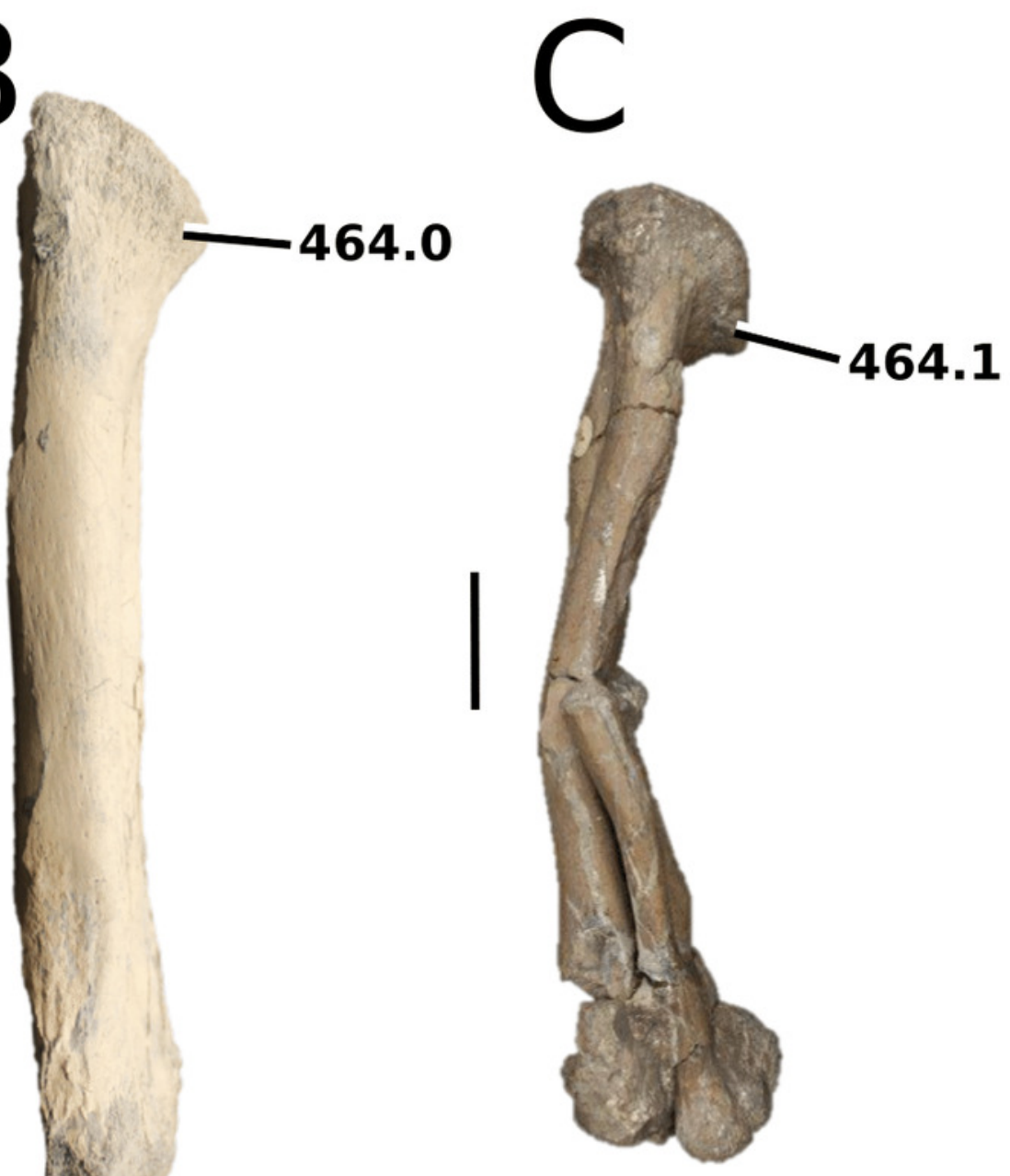


\section{Figure 50}

Comparative photographs: teleosauroid calcaneae and astragulae.

Figure 50. Comparative photographs of teleosauroid calcaneae and astragulae (ch. 466): Mycterosuchus nasutus (NHMUK PV R 2617) [(A-B) calcaneum in (A) dorsal and (B) lateral view; and (C) astragulus)], (D) Macrospondylus bollensis (SMNS 81699) and (E) Neosteneosaurus edwardsi (PETMG R175). Scale bars: $1.5 \mathrm{~cm}(\mathrm{~A}-\mathrm{C})$ and $2.5 \mathrm{~cm}(\mathrm{D}),(\mathrm{E})$ not to scale.
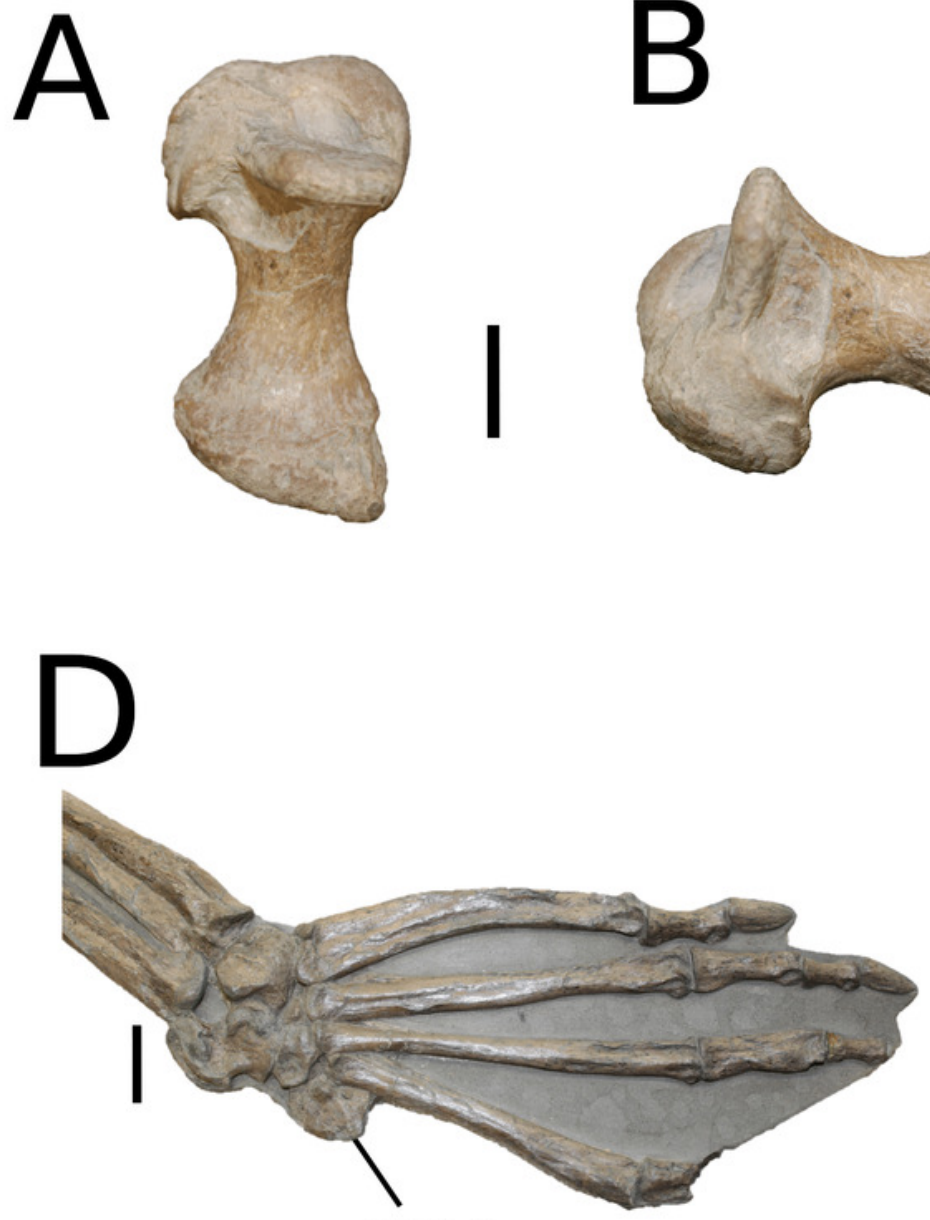

466.0
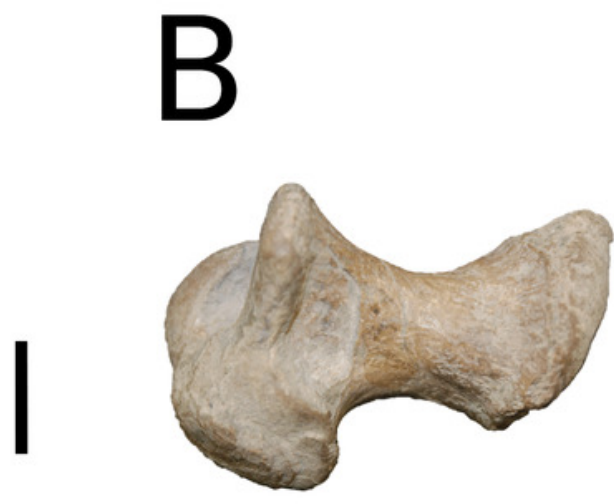

466.1
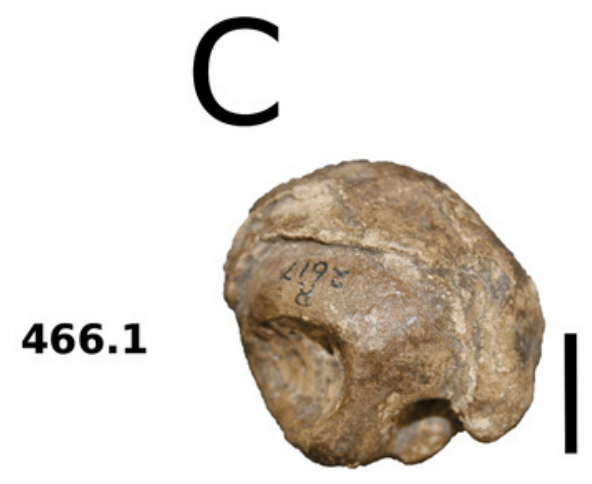


\section{Figure 51}

Comparative photographs: teleosauroid dorsal osteoderms.

Figure 51. Comparative photographs displaying teleosauroid dorsal sacral osteoderms, with emphasis on ornamentation pattern (ch. 473) and keel presence (ch. 489): (A) Plagiophthalmosuchus gracilirostris (NHMUK PV OR 14892), (B) Charitomenosuchus leedsi (NHMUK PV R 3806), (C) Teleosaurus cadomensis (NHMUK PV R 119a), (D) Mycterosuchus nasutus (NHMUK PV R 2617), (E) Neosteneosaurus edwardsi (PETMG R178), and (F) Lemmysuchus obtusidens (NHMUK PV R 3168). Scale bars: $3 \mathrm{~cm}$, (D) not to scale.
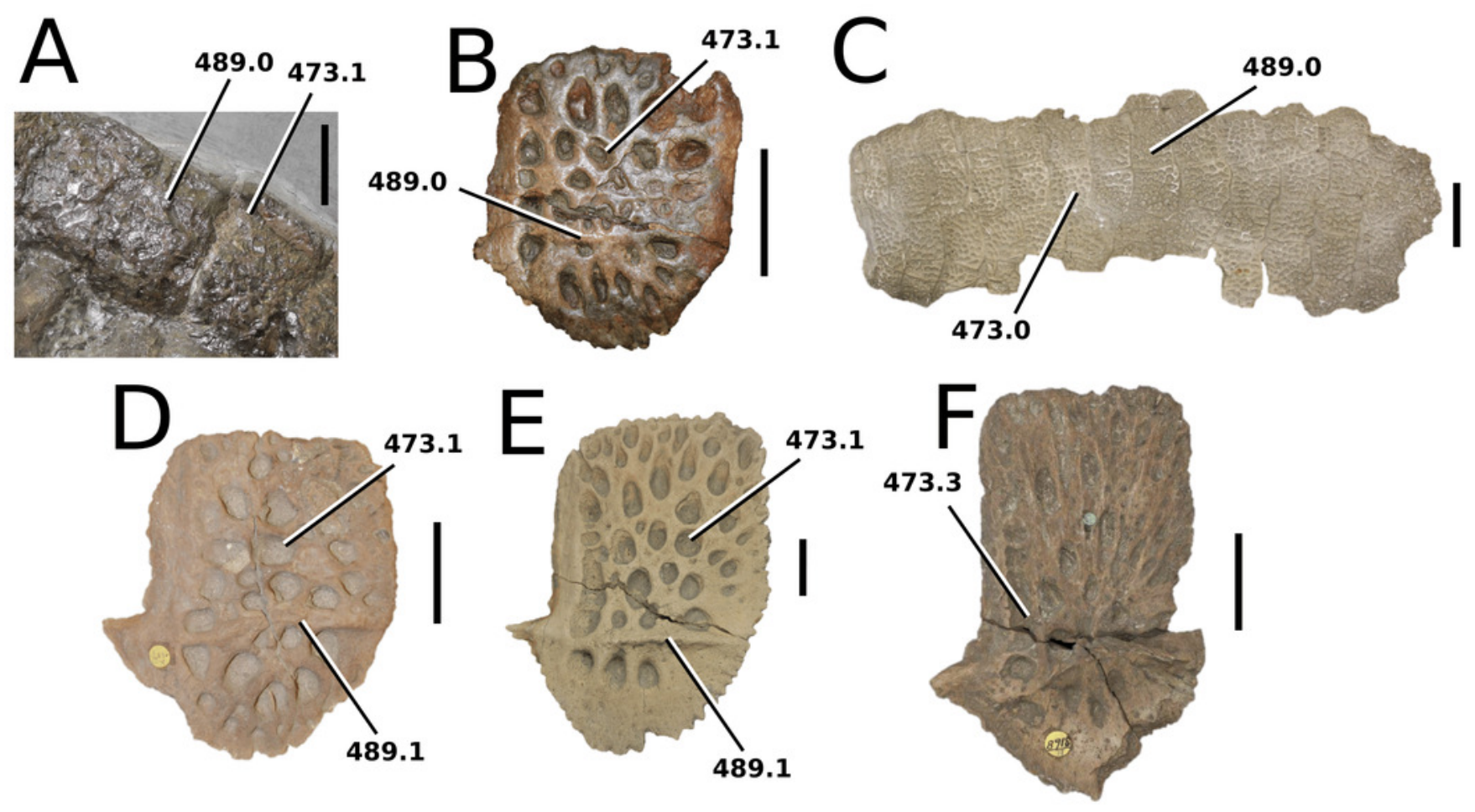


\section{Figure 52}

Comparative photographs: telescopic orbits and antorbital fenestrae.

Figure 52. Comparative photographs displaying telescopic orbits (ch. 10,151) as well as presence or absence (ch. 83) and shape of antorbital fenestrae (ch. 86) in dorsal view. (A) Plagiophthalmosuchus gracilirostris (NHMUK PV OR 14892); (B) Deslongchampsina larteti (OUMNH J.29851); (C) Indosinosuchus potamosiamensis (PRC-11); (D) Mycterosuchus nasutus (NHMUK PV R 2617); (E) Charitomenosuchus leedsi (NHMUK PV R 3806); (F) Yvridiosuchus boutilieri (OUMNH J.1401); (G) Proexochokefalos heberti (MNHN.F 1890-13); (H) Neosteneosaurus edwardsi (PETMG R178); and (I) Lemmysuchus obtusidens (LPP.M.21). Note the shallow antorbital fenestrae of $C$. leedsi compared to other taxa with antorbital fenestrae. Scale bars: $4 \mathrm{~cm}$. 


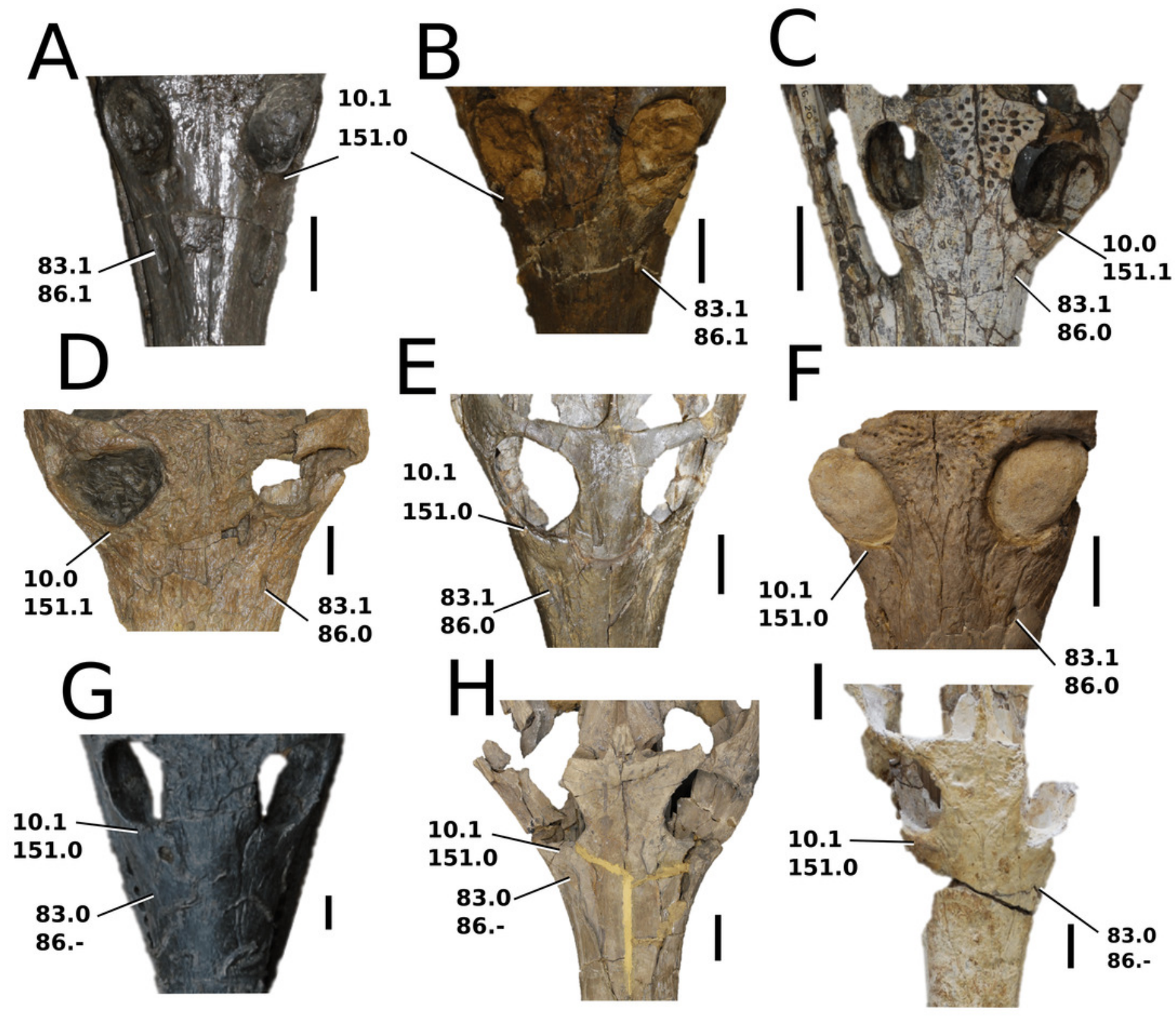




\section{Figure 53}

Comparative photographs: anterior and anterolateral premaxillary margins, and neurovascular foramina.

Figure 53. Comparative photographs displaying the anterior and anterolateral premaxillary margins (ch. 48) as well as neurovascular foramina (ch. 27), in lateral view: (A) Macrospondylus bollensis (SMNS 51563), (B) Mystriosaurus laurillardi (NHMUK PV OR 14781), (C) Machimosaurus buffetauti (SMNS 91415), (D) Lemmysuchus obtusidens (LPP.M.21), (E) Yvridiosuchus boutilieri (OUMNH J.1401) and (F) Indosinosuchus kalasinensis (PRC-239). Scale bars: $5 \mathrm{~cm}$.
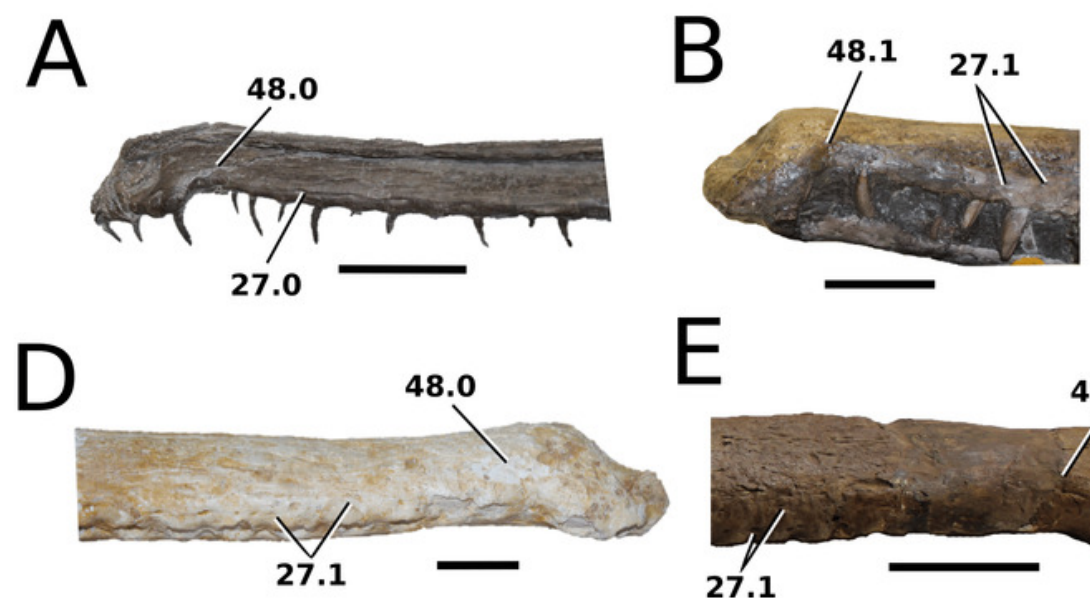

C
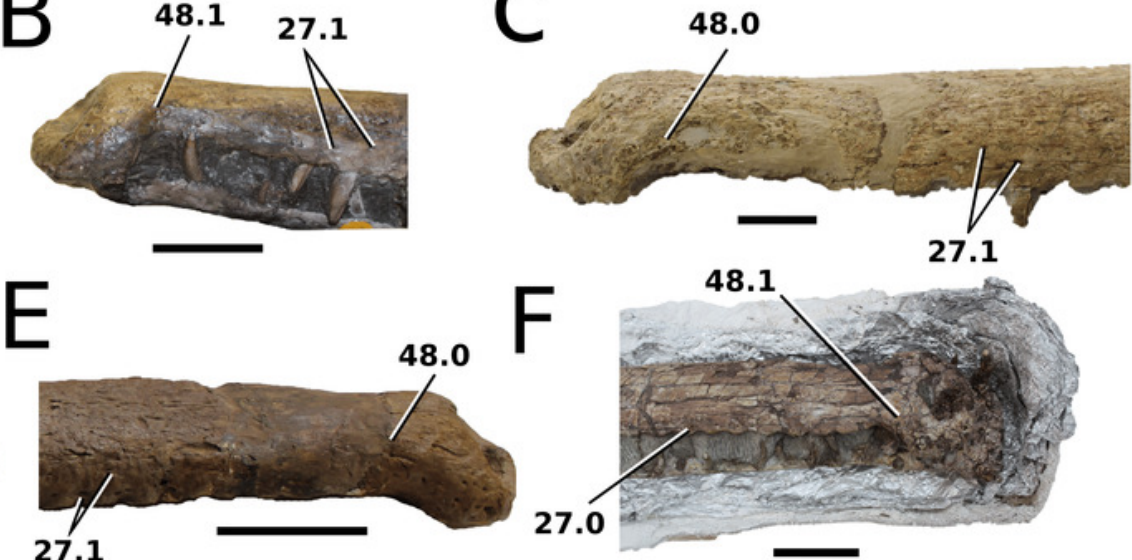

48.1
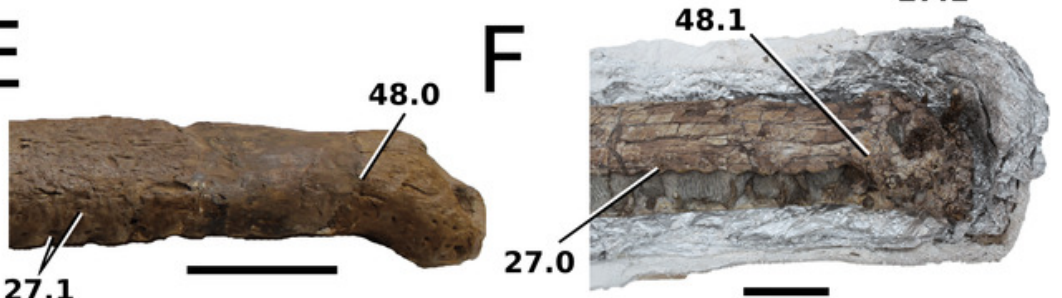


\section{Figure 54}

Comparative photographs: orientation of teleosauroid external nares.

Figure 54. Comparative photographs displaying the external nares, in dorsal view (ch. 34):

(A) the Chinese teleosauroid (IVPP V 10098), (B) Mystriosaurus laurillardi (HLMD V946-948),

(C) Bathysuchus megarhinus (unnumbered LPP specimen), (D) Deslongchampsina larteti

(OUMNH J.29851), (E) Neosteneosaurus edwardsi (NHMUK PV R 3701) and (F) Lemmysuchus obtusidens (LPP.M.21). Scale bars $3 \mathrm{~cm}$. 
A

34.0
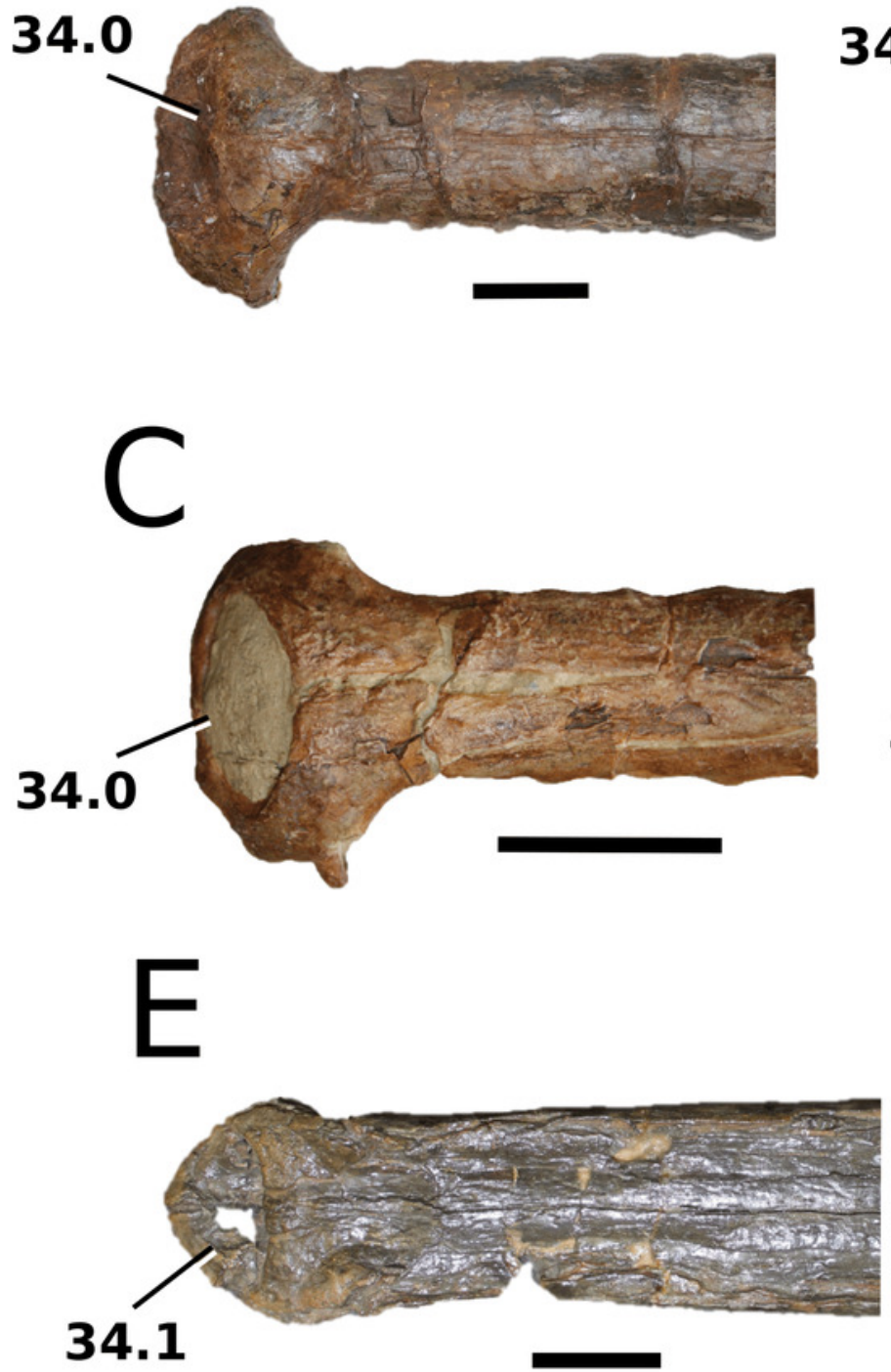

B

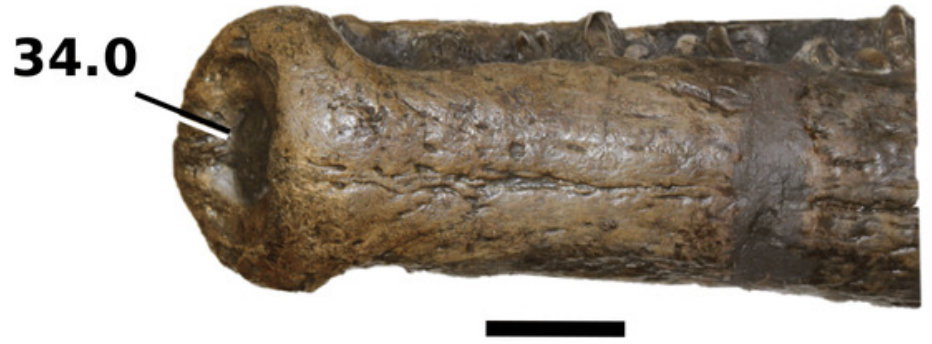

$\mathrm{D}$

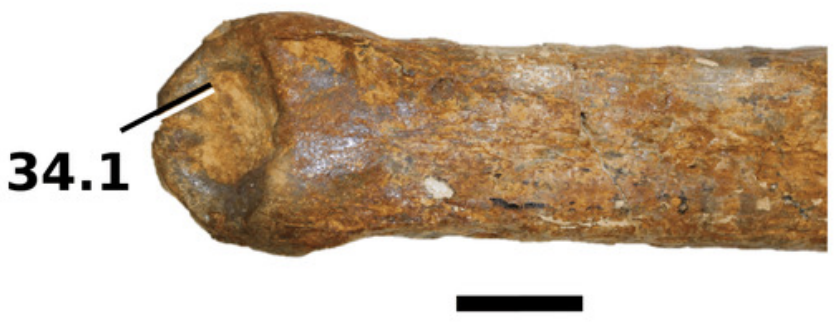

$\mathrm{F}$

34.1

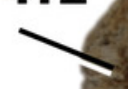




\section{Figure 55}

Comparative photographs: teleosauroid supratemporal fenestrae.

Figure 55. Comparative photographs displaying the shape of the supratemporal fenestrae (ch. 102), as well as the anterolateral expansion of the anterior portion (ch. 103) and elongation (ch. 104) of these fenestrae in dorsal view. (A) Teleosaurus cadomensis (MNHN AC 8746; (B) Mystriosaurus laurillardi (NHMUK PV OR 14781); (C) Plagiophthalmosuchus gracilirostris (NHMUK PV OR 14892); (D) Macrospondylus bollensis (MMG BwJ 565); (E) Clovesuurdameredeor stephani (NHMUK PV OR 49126), (F) Proexochokefalos heberti (MNHN.F 1890-13); and (G) Lemmysuchus obtusidens (NHMUK PV R 3168). Scale bars: $3 \mathrm{~cm}$ $(\mathrm{A}, \mathrm{C})$ and $10 \mathrm{~cm}(\mathrm{~B}, \mathrm{D}-\mathrm{F})$.

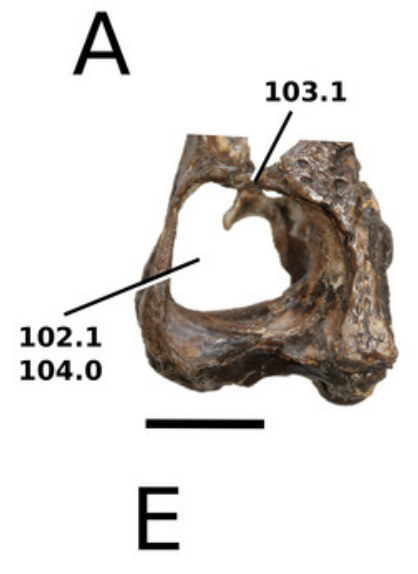

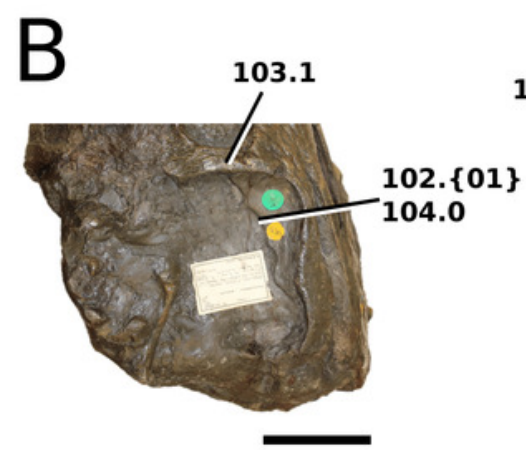
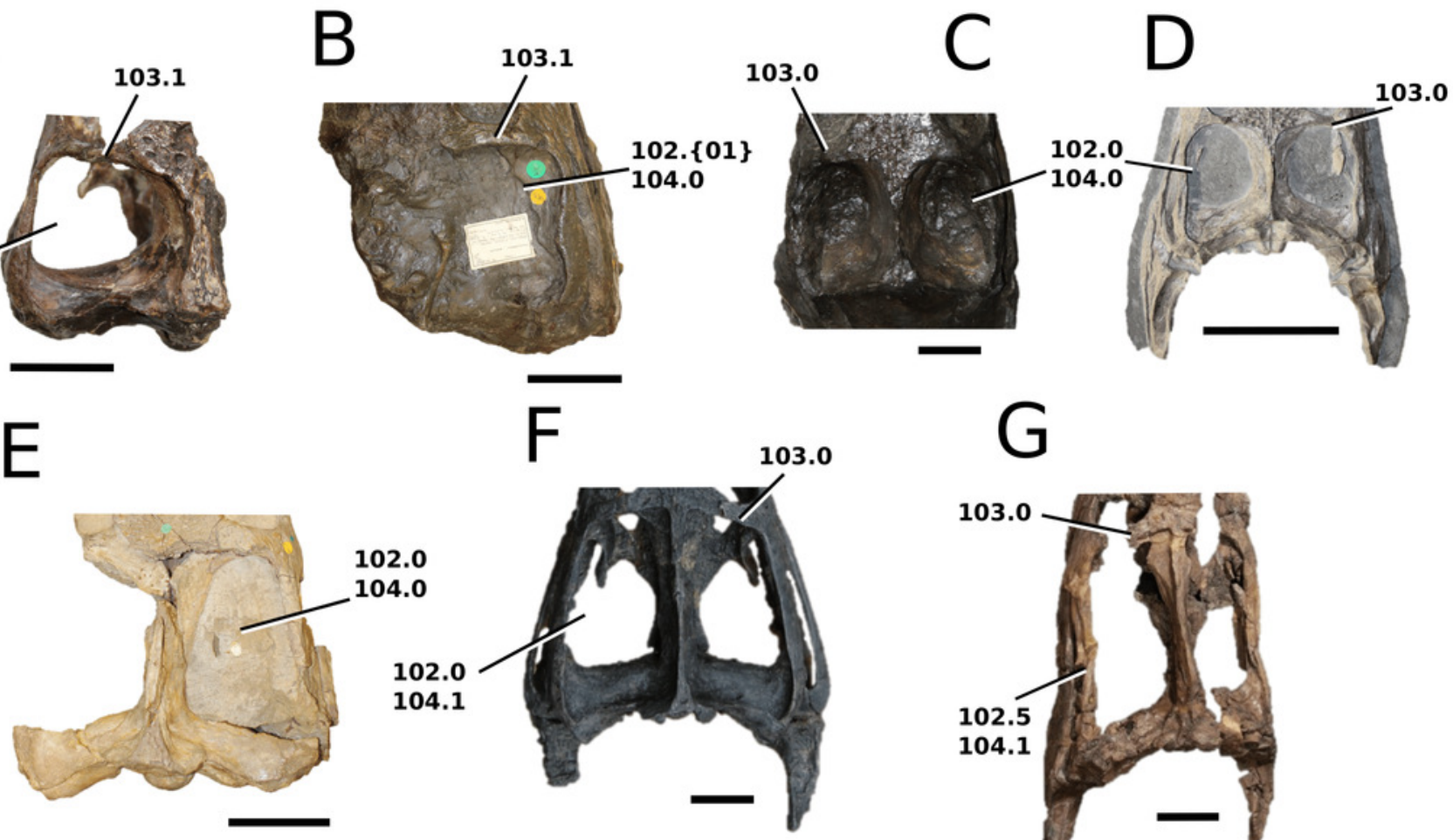

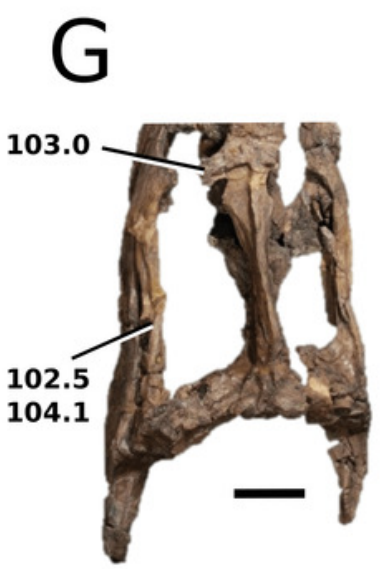




\section{Figure 56}

Comparative photographs: teleosauroid orbital margin.

Figure 56. Comparative photographs of teleosauroid orbital margin (in lateral view), focusing on the inclusion of the postorbital (ch. 158): (A) Plagiophthalmosuchus gracilirostris (NHMUK PV OR 14892), (B) Clovesuurdameredeor stephani (NHMUK PV OR 49126), (C) the Chinese teleosauroid (IVPP V 10098) and (D) Teleosaurus cadomensis (MNHN AC 8746). Scale bars: 3 $\mathrm{cm}$.

A

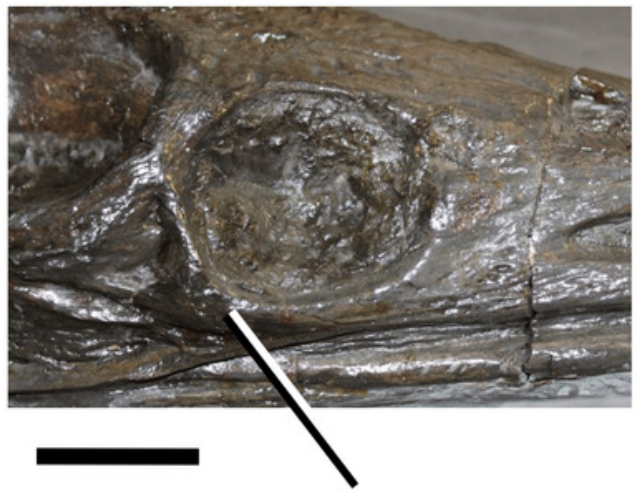

158.0

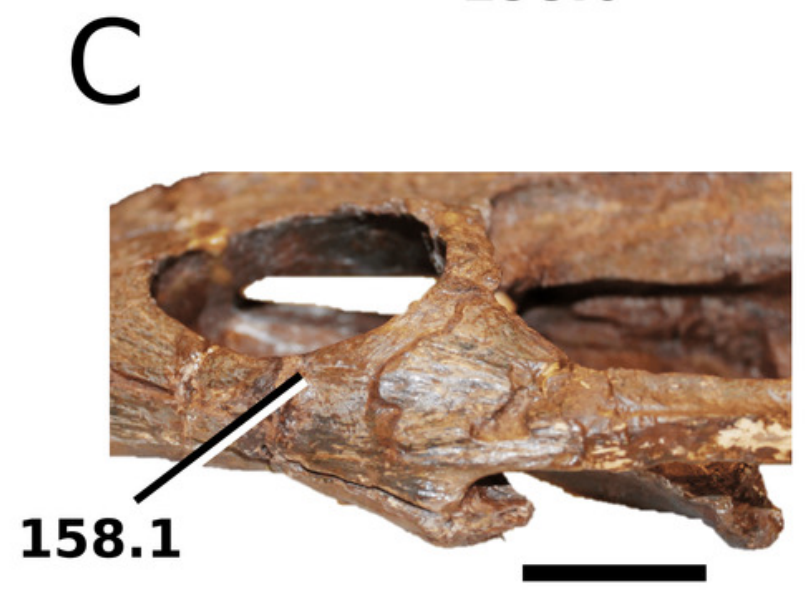

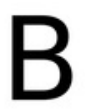
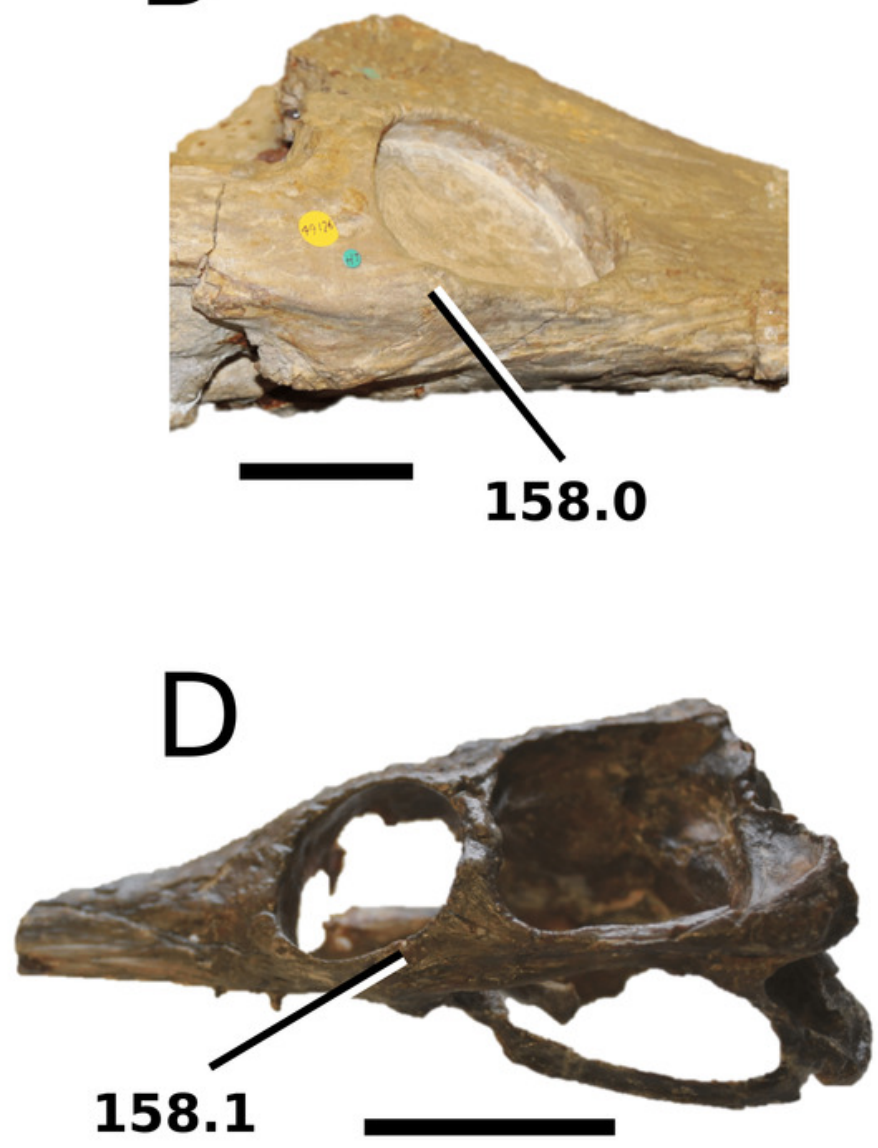


\section{Figure 57}

Comparative photographs: exposure of the teleosauroid basioccipital.

Figure 57. Comparative photographs exhibiting exposure of the teleosauroid basioccipital (ch. 225): (A) Mycterosuchus nasutus (CAMSM J.1420), (B) the Chinese teleosauroid (IVPP V 10098), (C) Charitomenosuchus leedsi (NHMUK PV R 3320) and (D) Neosteneosaurus edwardsi (NHMUK PV R 2865). Scale bars: $7 \mathrm{~cm}$.
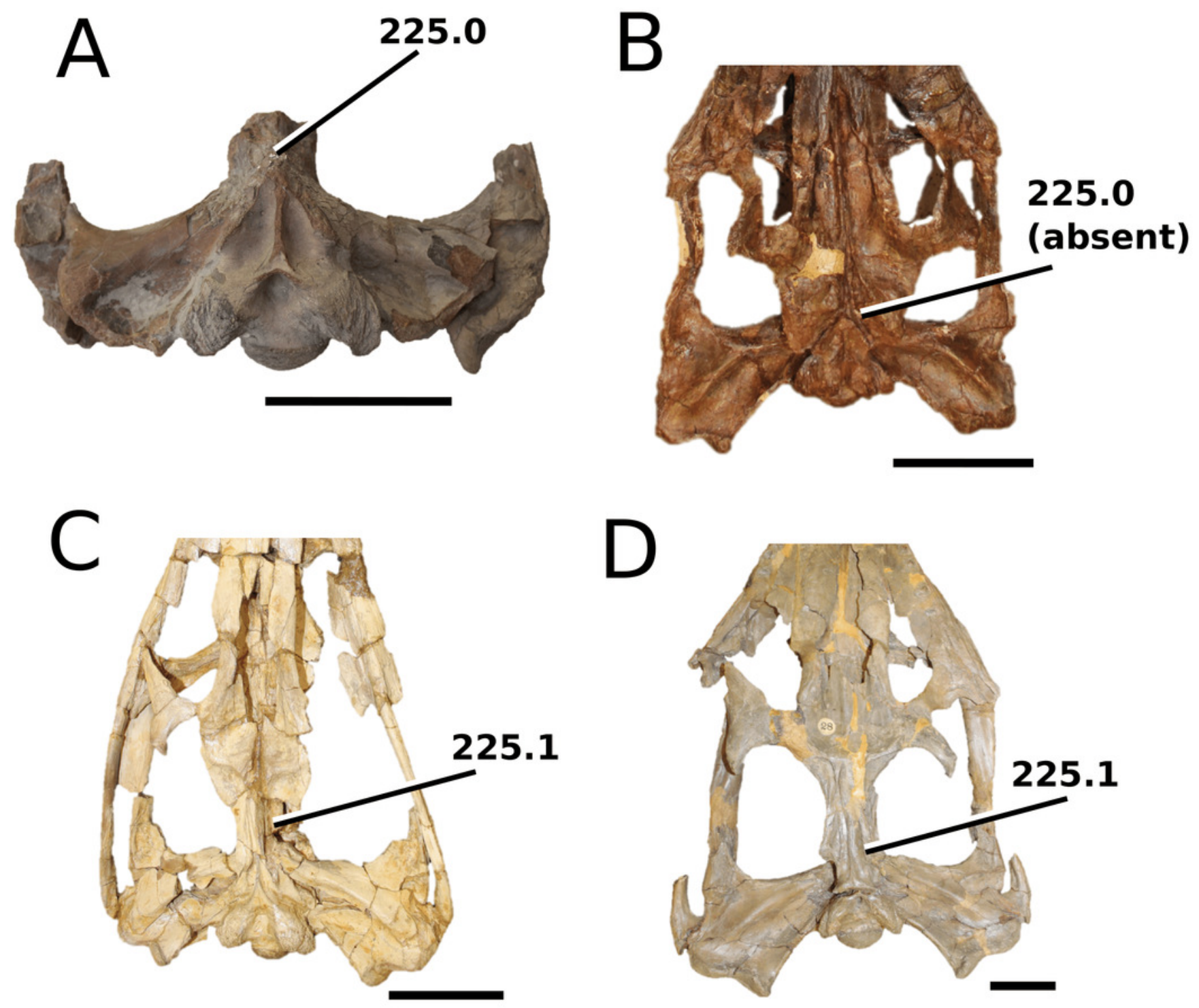


\section{Figure 58}

Comparative photographs: teleosauroid humeri.

Figure 58. Comparative photographs of teleosauroid humeri (ch. 410): (A) Mycterosuchus nasutus (NHMUK PV R 2617), (B) Macrospondylus bollensis (SMNS 18672), (C) Neosteneosaurus edwardsi (NHMUK PV R 3701), (D) Charitomenosuchus leedsi (NHMUK PV R 3806) and (E) Aeolodon priscus (MNHN.F.CNJ 78). Scale bars: $3 \mathrm{~cm}$. (ch. 410):

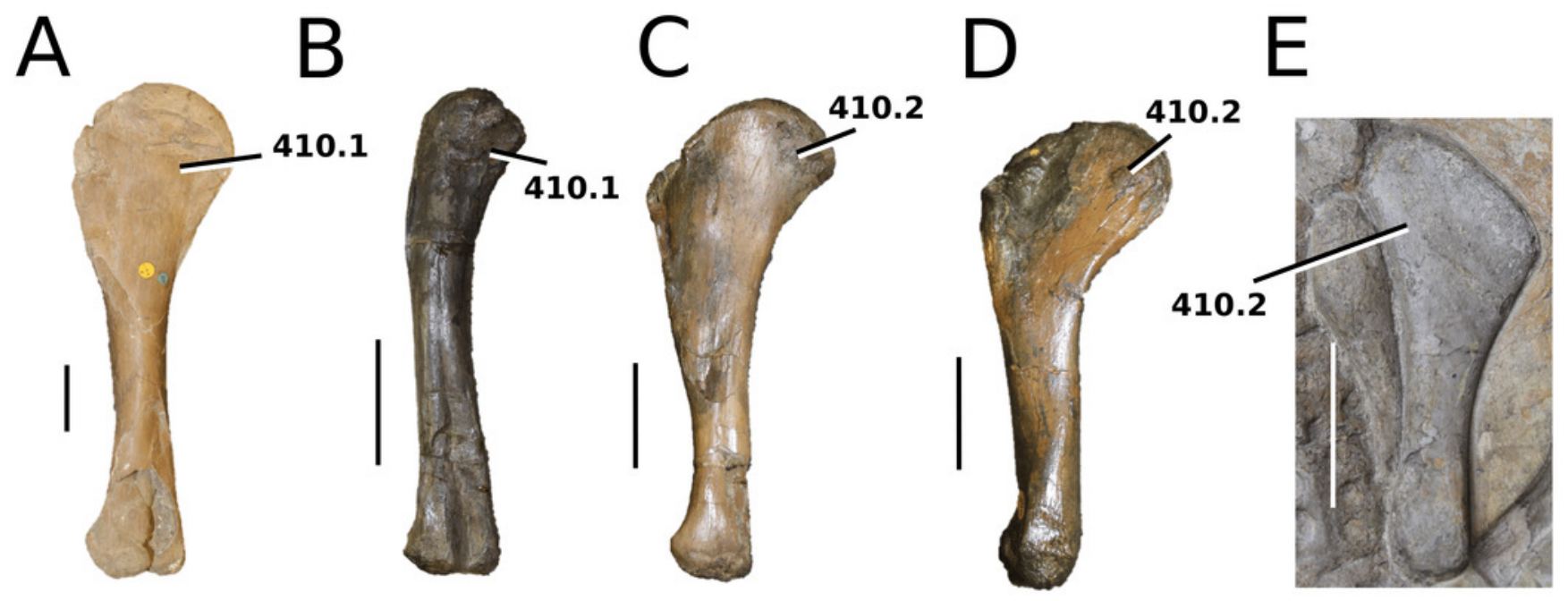




\section{Figure 59}

Topologies of the unweighted parsimonious phylogenetic analysis.

Figure 59. Results of the unweighted parsimonious phylogenetic analysis, focusing on Teleosauroidea. (A) simplified strict consensus topology (125 MPTs and 1659 steps: $\mathrm{Cl}=$ $0.405, \mathrm{RI}=0.844)$; (B) simplified strict consensus topology excluding S. rostromajor (176 MPTs and 1659 steps: $\mathrm{Cl}=0.405, \mathrm{RI}=0.844$ ); and $(C)$ parsimonious majority rules topology (125 MPTs and 1659 steps). In all topologies Teleosauroidea is monophyletic and two distinct families ( $T$ and $M$ ) are recovered. Bremer support and jackknife values (Bremer/jackknife; AB) and support percentages (C) are included. 
A

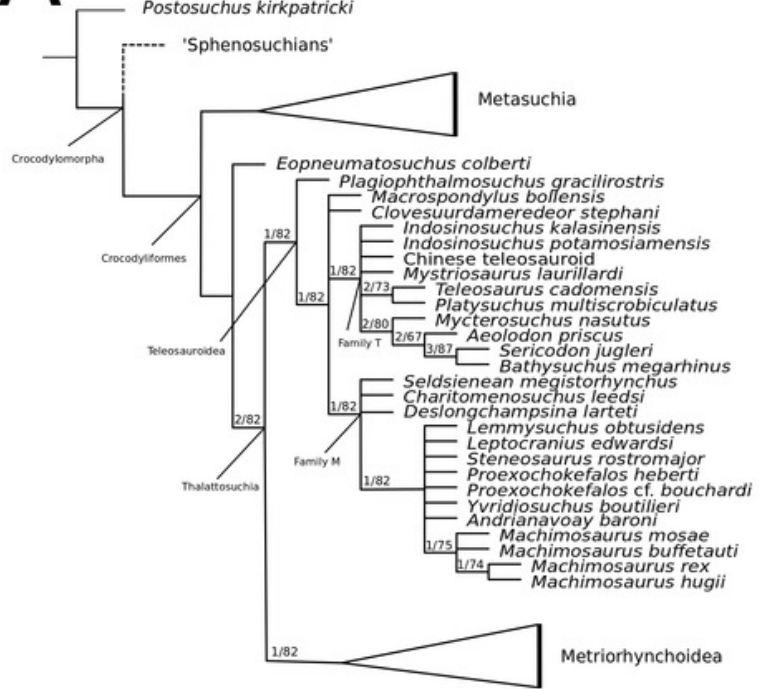

B

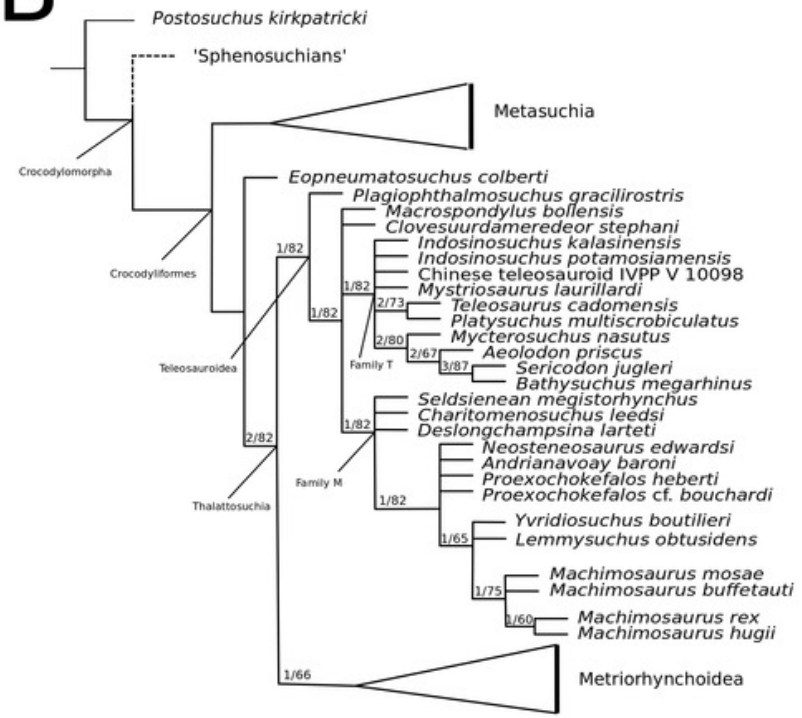

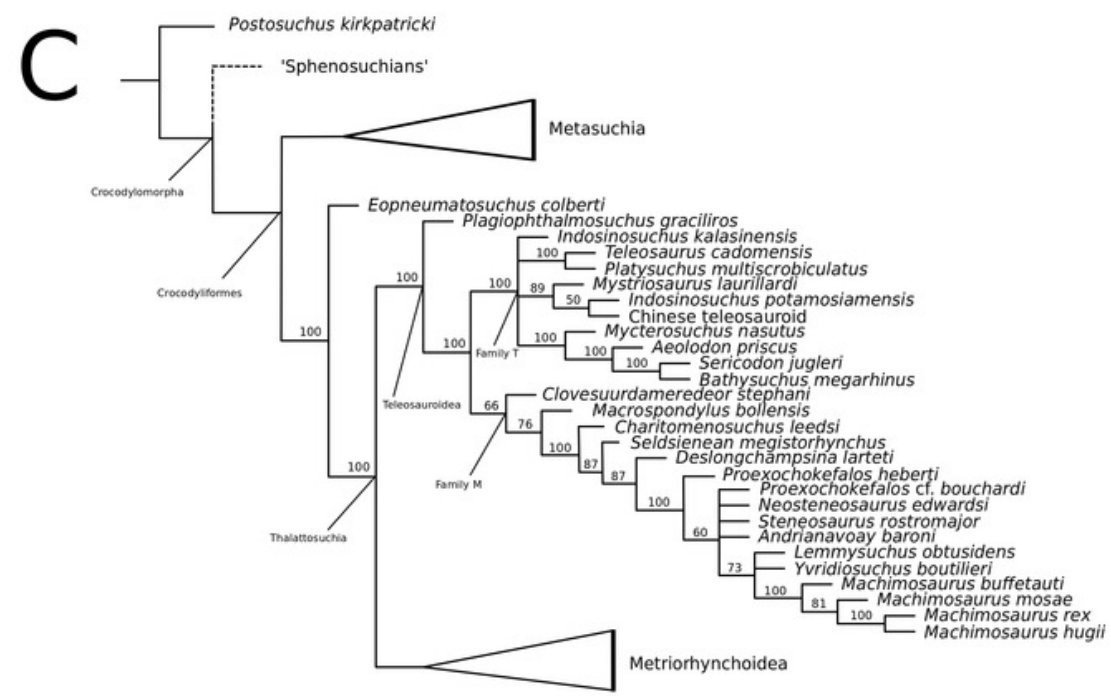




\section{Figure 60}

Topologies of the EIW parsimonious phylogenetic analysis, in addition to the maximum agreement subtree.

Figure 60. Results of the extended weighted parsimonious phylogenetic analysis, focusing on Teleosauroidea. (A) Simplified strict consensus topology with extended implied weighting $(k=12)$ of the 47 MPTs; (B) simplified strict consensus topology with extended implied weighting ( $\mathrm{k}=12$ ) excluding S. rostromajor (39 MPTs); and (C) agreement subtree (based on the unweighted strict consensus) of Teleosauroidea.

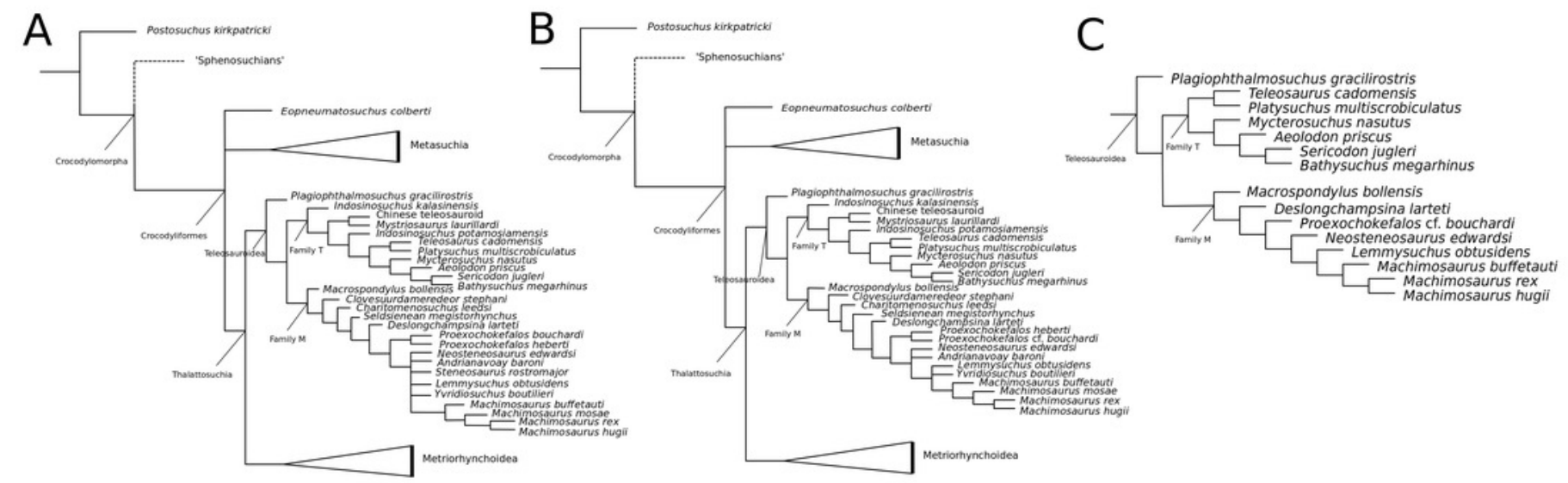




\section{Figure 61}

Simpli $\square$ ed consensus topology in MrBayes.

Figure 61. Simpli $\square$ ed consensus topology, produced in MrBayes using gamma distribution

(rates $=$ gamma), standard deviation $=0.019863$, harmonic mean $=-7785.47$. Note that $S$. rostromajor is recovered as most closely related to Machimosaurini.

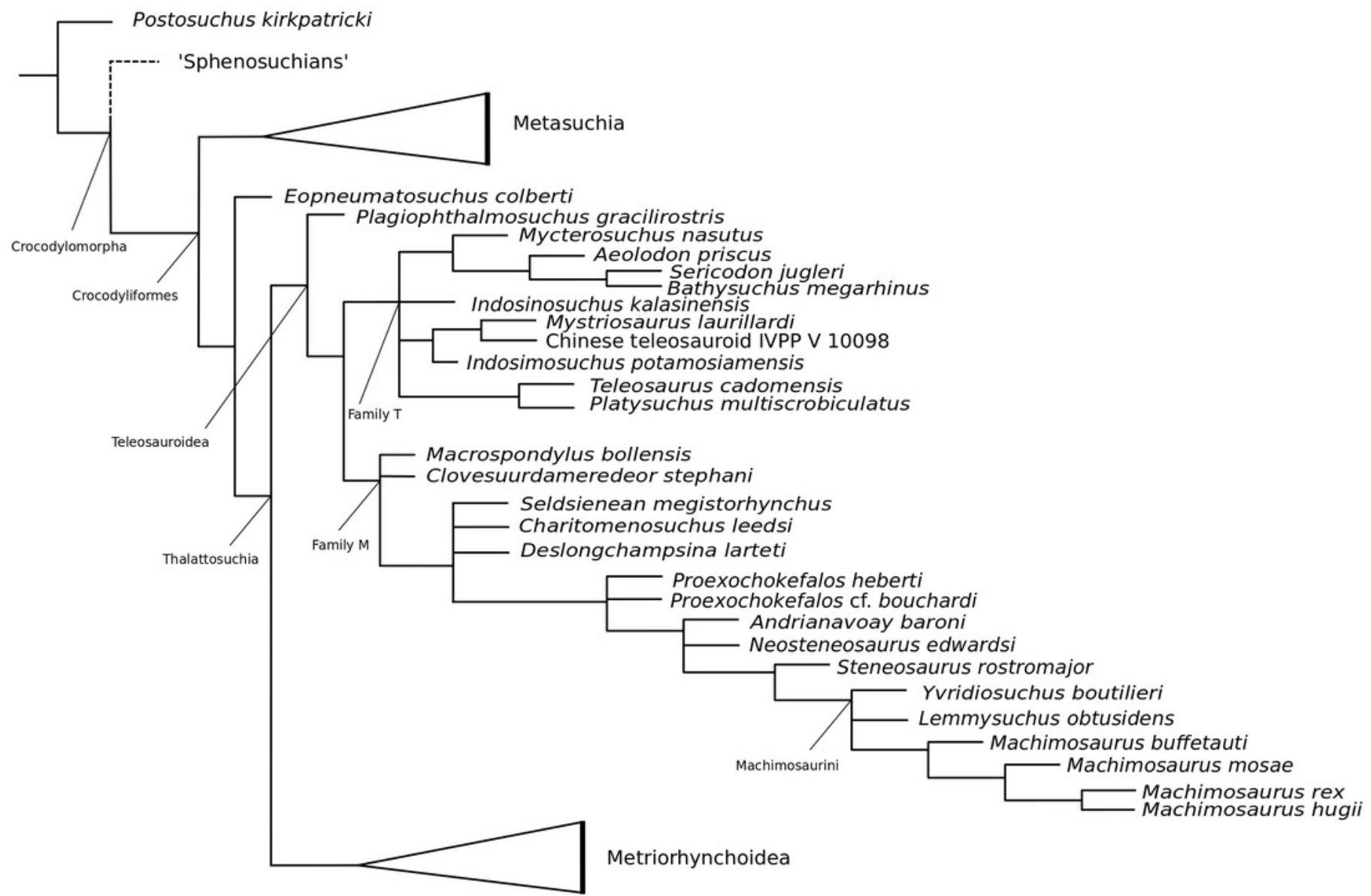




\section{Figure 62}

Three excluded teleosauroid taxa from our dataset.

Figure 62. Photographs of three well preserved taxa not included in our dataset: (A) Steneosaurus pictaviensis (= Charitomenosuchus leedsi) LPP.M.37; (B) Steneosaurus depressus (= Proexochokefalos heberti) OUMNH J.01420; and (C) Steneosaurus hulkei (= Neosteneosaurus edwardsi) (NHMUK PV R 2074). See text for in-depth explanation as to why these taxa are excluded. Scale bars: $4 \mathrm{~cm}(\mathrm{~A}, \mathrm{C})$ and $10 \mathrm{~cm}(\mathrm{~B})$. 


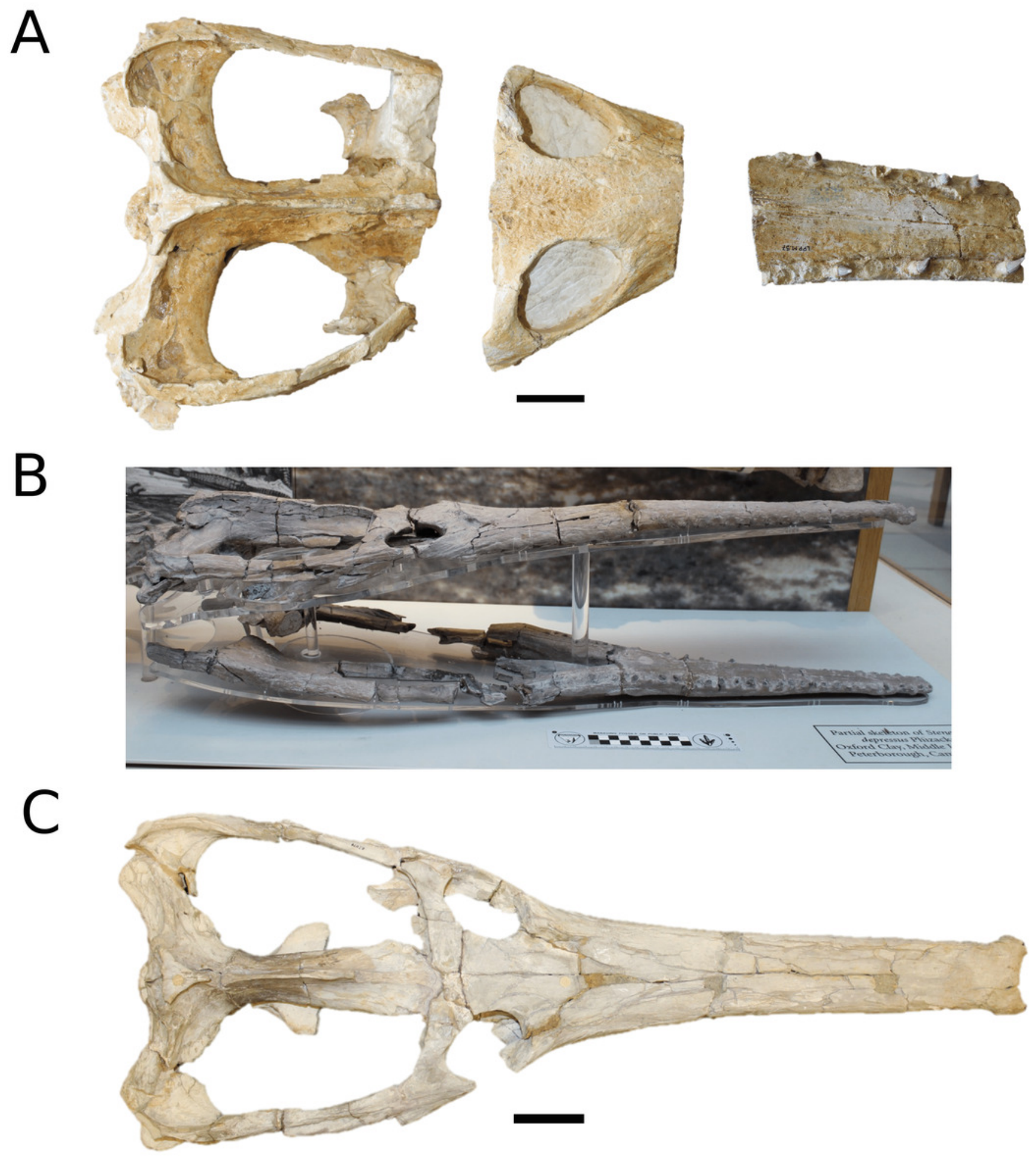




\section{Figure 63}

Teleosauroid hypothesized ecomorphologies indicated on the extended implied weighted topology.

Figure 63. Hypothesized teleosauroid ecomorphologies mapped onto the extended implied weighted topology (excluding Steneosaurus rostromajor: $39 \mathrm{MPTs}$ ): (A) rostral morphology; (B) feeding ecology; and (C) palaeohabitat. Note that Family $\mathrm{T}$ is more phenotypically plastic than Family $M$ in terms of (A) rostrum and (C) habitat, and that Family M shows a distinctive, linear shift in (A) rostral length and (B) feeding style. 


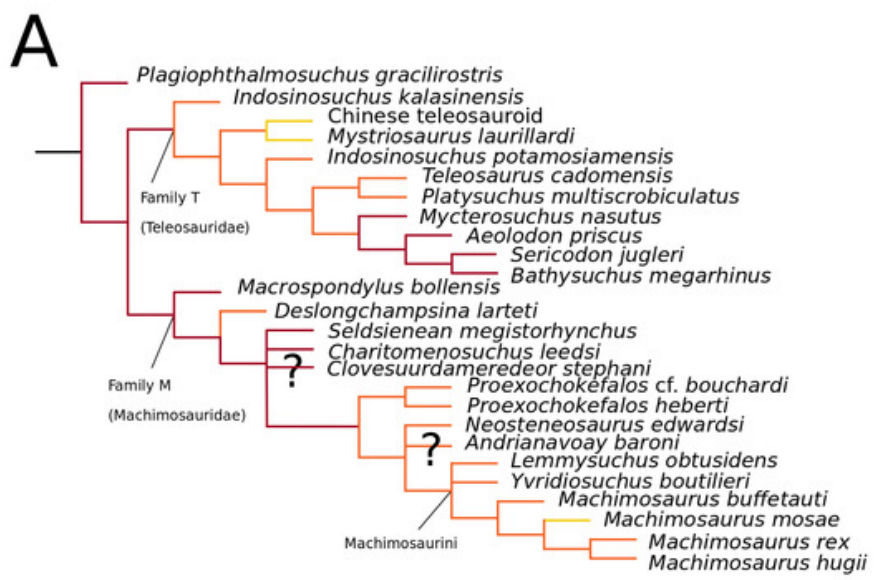

B
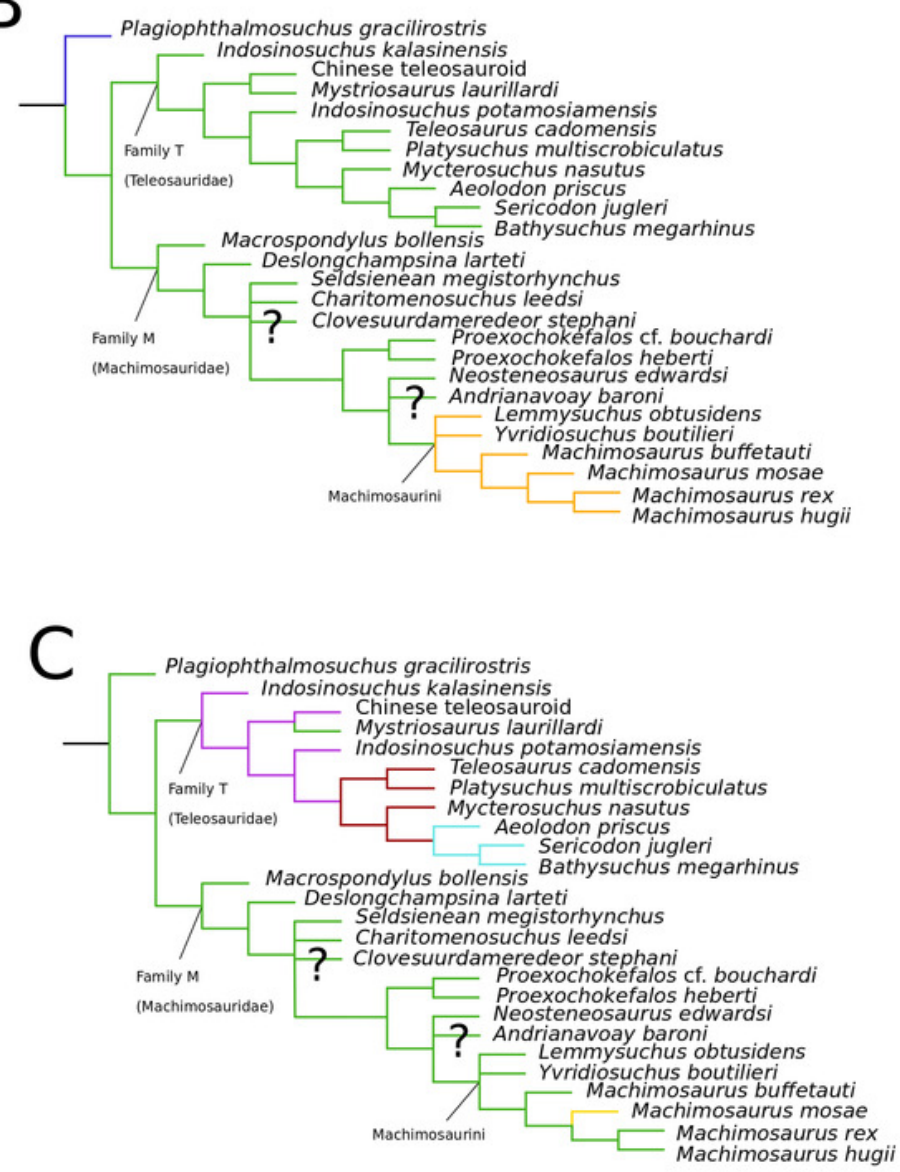

\section{LEGEND: ROSTRUM}

longirostrine

mesorostrine

brevirostrine

\section{LEGEND: FEEDING}

specialist

generalist

macrophagous/

durophagous

\section{LEGEND: HABITAT}

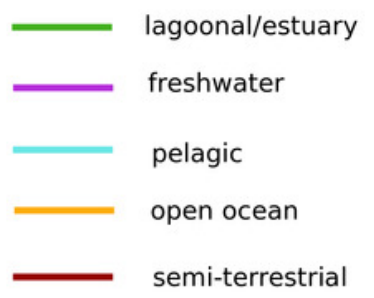




\section{Figure 64}

Time calibrated tree

Figure 64. Summary of time-calibrated phylogeny (extended implied weighting excluding Steneosaurus rostromajor: 39 MPTs) of teleosauroids, focusing on number (n】) of ecomorphological guilds present during four main time periods (Toarcian, Bathonian, Callovian and Kimmeridgian). Major guilds are as follows: dark blue = longirostrine specialist; purple $=$ mesorostrine generalist; light blue $=$ pelagic generalist; black $=$ longirostrine generalist; yellow = macrophagous/durophagous; red = semi-terrestrial generalist. Grey coloured lines indicate unknown ecomorphology, due to incomplete material. Note that the number of guilds remains constant (four) until the Kimmeridgian, in which there is a drop (three). Silhouettes provided by PhyloPic (by G. Monger, S. Hartman and N. Tamara).

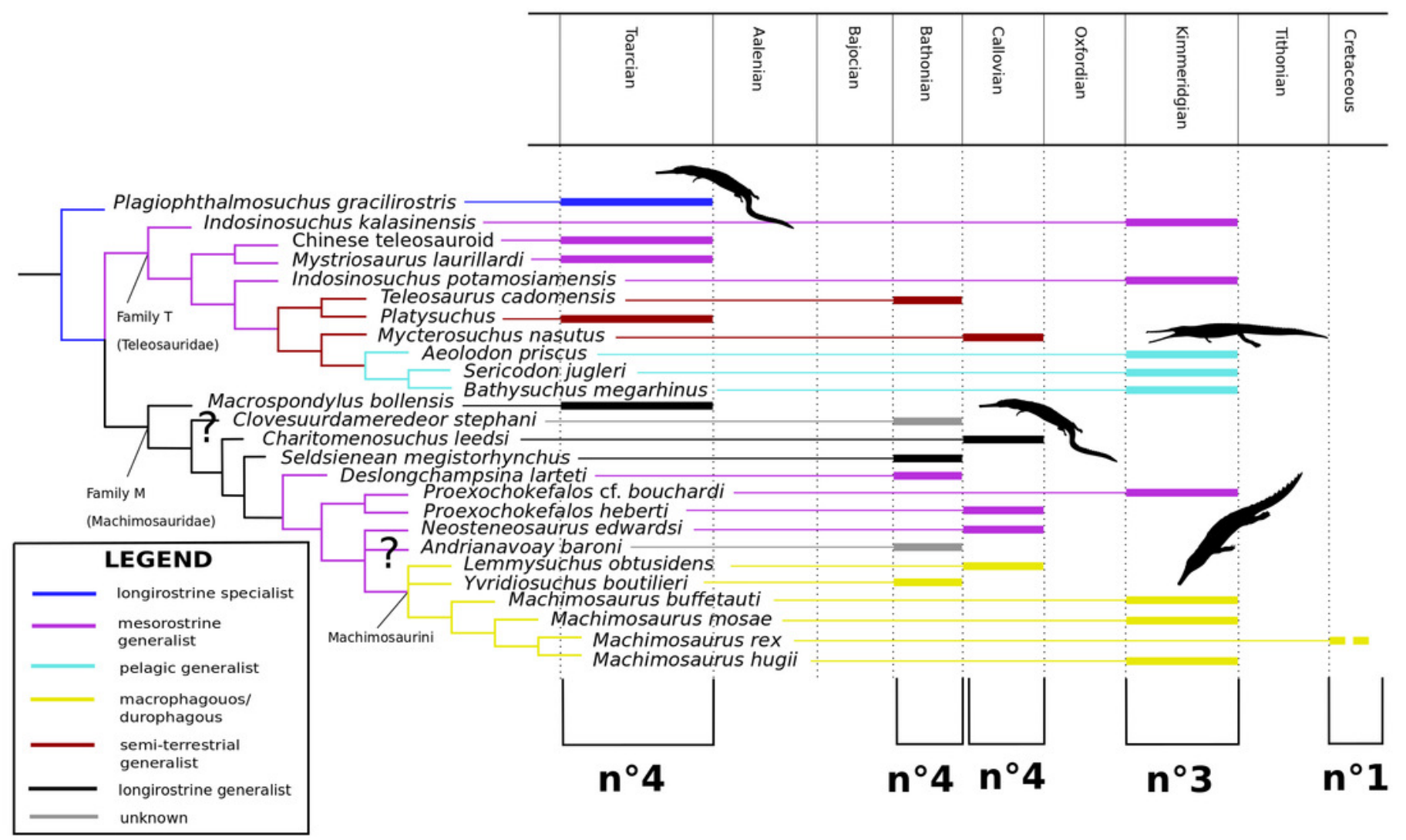

